\title{
Groupoids in categories with partial covers
}

\author{
Dissertation
}

zur Erlangung des mathematisch-naturwissenschaftlichen Doktorgrades

"Doctor rerum naturalium" (Dr.rer.nat.)

der Georg-August-Universität Göttingen

im Promotionsprogramm der PhD School of Mathematical Science (SMS)

der Georg-August University School of Science (GAUSS)

vorgelegt von

Giorgi Arabidze

aus Tiflis, Georgien

Göttingen 2018 
Betreungsausschuss:

Betreuer: Prof. Dr. Ralf Meyer

Mathematisches Institut, Georg-August-Universität Göttingen

Betreuerin: Prof. Dr. Chenchang Zhu

Mathematisches Institut, Georg-August-Universität Göttingen

\section{Mitglieder der Prüfungskommission:}

Referent: Prof.Dr. Ralf Meyer

Mathematisches Institut, Georg-August-Universität Göttingen

Koreferentin: Prof. Dr. Chenchang Zhu

Mathematisches Institut, Georg-August-Universität Göttingen

Prof. Dr. Preda Mihailescu

Mathematisches Institut, Georg-August-Universität Göttingen

Prof. Dr. Thomas Schick

Mathematisches Institut, Georg-August-Universität Göttingen

Prof. Dr. Dominic Schuhmacher

Institut für Mathematische Stochastik, Georg-August-Universität Göttingen

Prof. Dr. Max Wardetzky

Institut für Numerische und Angewandte Mathematik, Georg-August-Universität Göttingen

Tag der mündlichen Prüfung: 15.10.2018 


\section{CONTENTS}

1. Introduction 3

2. Pretopologies 6

2.1. Extra assumptions on stronger pretopologies 9

3. Groupoids in a category with partial covers 10

3.1. Examples of groupoids 15

4. Morphisms between groupoids 16

\begin{tabular}{lll}
\hline 4.1. Groupoid fibrations & 18
\end{tabular}

$\begin{array}{lll}5 . & \text { Groupoid actions } & 28\end{array}$

5.1. Examples of groupoid actions 33

\begin{tabular}{lll}
\hline 5.2. & Transformation groupoids & 35
\end{tabular}

\begin{tabular}{lll}
\hline 5.3. & Principal bundles & 39
\end{tabular}

\begin{tabular}{lll}
\hline 5.4. & Examples of principal bundles & 40
\end{tabular}

$\begin{array}{lll}5.5 . & \text { Pull-back of a bundle } & 42\end{array}$

$\begin{array}{lll}5.6 . & \text { Basic actions and assumptions on it } & 47\end{array}$

5.7. Groupoid fibrations with basic fibre 50

6. Generalised morphisms between groupoids 56

6.1. Actors 56

$\begin{array}{lll}6.2 . & \text { Bibundle actors } & 57\end{array}$

$\begin{array}{lll}\text { 6.3. } & \text { Bibundle functor } & 61\end{array}$

$\begin{array}{lll}6.4 . & \text { Bibundle equivalence } & 66\end{array}$

\begin{tabular}{lll}
\hline 7. Generalised groupoid actions & 68
\end{tabular}

8. Examples of categories with partial covers 81

$\begin{array}{lll}\text { 8.1. Category of sets } & 82\end{array}$

8.2. Categories of topological spaces $\quad 82$

\begin{tabular}{lll}
\hline 8.3. Categories of manifolds & 89
\end{tabular}

$\begin{array}{ll}\text { References } & 90\end{array}$ 


\section{INTRODUCTION}

Groupoids are basic objects in noncommutative geometry and differential geometry. There are different kinds of groupoids, such as topological groupoids and Lie groupoids of infinite or finite dimension. Meyer and Zhu 47] developed a framework to study these different kinds of groupoids with the same theory. More recently, it became clear that this framework should be modified to allow for various "partial" phenomena, such as partial actions, partial groupoid equivalences, or partial fibrations. This thesis develops this variant of the theory in [47.

The notion of groupoid has many meanings. It depends on in which field of mathematics it is considered. For instance, there are topological groupoids, étale topological groupoids, Lie groupoids of finite and infinite dimension, algebraic groupoids, and so on. The paper of Meyer and Zhu [47] developers a common theory for these different kinds of groupoids. For instance, they construct a bicategory of groupoids with bibundle equivalences as arrows and equivariant maps as 2arrows. A bibundle equivalence is also called a Morita equivalence. A Morita equivalence of locally compact, Hausdorff topological groupoids is defined by Muhly, Renault and Williams in [39]. They proved that Morita equivalent locally compact, Hausdorff groupoids have equivalent $C^{*}$-algebras. Hilsum and Skandalis define a generalised morphism between Lie groupoids in [6] in order to build wrong-way maps in the K-theory of foliation $C^{*}$-algebras. Lie groupoids with isomorphism classes of Hilsum-Skandalis morphisms as arrows form a category [18. Bibundle functors are introduced by Meyer and Zhu in [47. They are an abstract analogue of Hilsum-Skandalis morphisms. Meyer and Zhu construct a bicategory of groupoids with bibundle functors as arrows and equivariant maps as 2-arrows.

A pretopology is an extra structure in a general category that allows to develop various kinds of mathematical objects. For instance, groupoids, groupoid actions, principal bundles, groupoid fibrations, actors, Hilsum-Skandalis morphisms, Morita equivalences, and so on. A category with pretopology is equipped with a notion of "cover". As it is discused above there are many different kinds of groupoids. In each case, the range and source maps are assumed to be "covers". For instance, covers are surjective submersions in the context of Lie groupoids. The covers also influence the notion of principal bundle because their bundle projections are assumed to be covers; this is equivalent to "local triviality" in the sense of the pretopology. If our category is that of topological spaces and the covers are the continuous surjections with local continuous sections, then we get exactly the usual notion of local triviality for principal bundles; this is why many geometers prefer this pretopology on topological spaces. Many operator algebraists prefer the pretopology of open continuous surjections instead.

In the abstract setting of groupoids in a category with pretopology there occurred the importance to modify the categorical framework there to allow for "partial" notions. A category with partial covers is equipped with a notion of "partial cover", which allows to define partial sheaves, partial bibundle actors, partial Hilsum-Skandalis morphisms, partial Morita equivalences, and so on.

The book proposed by Ruy Exel is about partial dynamical systems, [49]. A partial action of a group is an important notion in this theory and it is largely connected to some basic tools in this thesis. Also, Ralf Meyer and Alcides Buss define and study partial fibrations of topological groupoids. This notion of a groupoid fibration comes from higher category theory. It can also be defined in a category with partial covers and several basic properties can be proved in this general situation. If 
the partial covers are étale continuous maps, partial bibundle actors are the groupoid correspondences which are introduced by Suliman Albandik in his dissertation.

Ralf Meyer and Alcides Buss define different kinds of morphisms between topological groupoids. They call a continuous functor $\mathrm{F}: \mathrm{L} \rightarrow \mathrm{H}$ a fibration of topological groupoids if the continuous map

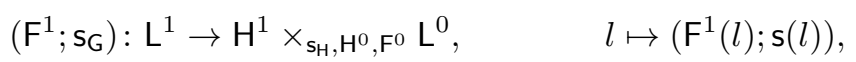

is an open surjection. They call the functor $\mathrm{F}: \mathrm{L} \rightarrow \mathrm{H}$ a groupoid covering if (1.1) is an isomorphism. The fibre of this fibration of topological groupoids is the subgroupoid $G$ of $L$ defined by $\mathrm{G}^{0}=\mathrm{L}^{0}$ and

$$
\mathrm{G}^{1}=\left\{g \in \mathrm{L}^{1} \mid \mathrm{F}^{1}(g)=1_{\mathrm{F}^{0}\left(\mathrm{~s}_{\mathrm{G}}(g)\right.}\right\},
$$

equipped with the subspace topology on $\mathrm{G}^{1} \subseteq \mathrm{L}^{1}$. They prove that many properties are preserved by fibrations, such as being (locally) Hausdorff, locally compact, amenable, étale or proper. They show that a crossed product for an action of $\mathrm{L}$ is isomorphic to an iterated crossed product first by $\mathrm{G}$ and then by $\mathrm{H}$. Here "groupoid action" means a saturated Fell bundle over the groupoid, and "crossed product" means the section $C^{*}$-algebra. They interpret a (partial) fibration of topological groupoids $\mathrm{L} \rightarrow \mathrm{H}$ with fibre $\mathrm{G}$ as a generalised (partial) action of $\mathrm{H}$ on $G$ by groupoid equivalences. The idea is the following: An action of $H$ on $G$ should give a transformation groupoid $H:=G \rtimes H$ that contains $\mathrm{G}$ and comes with a continuous functor $\mathrm{L} \rightarrow \mathrm{H}$. Thus defining actions of topological groupoids on topological groupoids amounts to characterising which chains of continuous functors $\mathrm{G} \hookrightarrow \mathrm{L} \rightarrow \mathrm{H}$ correspond to actions. They require $\mathrm{L} \rightarrow \mathrm{H}$ to be a groupoid fibration with fibre G.

Groupoid fibrations are inspired by higher category theory. The thesis of Li Du describes actions of $\infty$-groupoids on $\infty$-groupoids through Kan fibrations in [54]. By definition, a groupoid fibration between two topological groupoids is a Kan fibration between the associated topological $\infty$-groupoids.

There is the well known decomposition $C^{*}(X \rtimes \mathrm{H}) \cong C^{0}(X) \rtimes \mathrm{H}$ for an action of a groupoid $\mathrm{H}$ on a space $X$. Meyer and Buss generalise this fact. If we have a groupoid fibration $\mathrm{G} \hookrightarrow \mathrm{L} \rightarrow \mathrm{H}$, then there is an induced action of $\mathrm{H}$ on the $C^{*}$-algebra of $\mathrm{G}$, such that the cross product is $C^{*}(\mathrm{~L})$. In general, an "action" of a locally compact groupoid on a $C^{*}$-algebra is a (saturated) Fell bundle over the groupoid, and its "crossed product" is the section $C^{*}$-algebra of the Fell bundle. Saturated Fell bundles are interpreted as actions by Morita-Rieffel equivalences.

A (partial) groupoid fibration, (partial) groupoid covering and the fibre can be defined in a category with partial covers (see Definitions 4.6 and 4.13 and Proposition 4.15). We use the notation $\mathrm{G} \hookrightarrow \mathrm{L} \rightarrow \mathrm{H}$ to denote that we have a partial groupoid fibration from $\mathrm{L}$ to $\mathrm{H}$ with fibre $\mathrm{G}$. We generalise some basic results about (partial) groupoid fibrations and their fibres. The fibre of a (partial) groupoid fibration $\mathrm{F}: \mathrm{L} \rightarrow \mathrm{H}$ is a 0 -groupoid (groupoid where the range map is an isomorphism) if and only if $\mathrm{F}: \mathrm{L} \rightarrow \mathrm{H}$ is a (partial) groupoid covering. The composition of (partial) groupoid fibrations is a (partial) groupoid fibration. The composable pair of (partial) groupoid fibrations $\mathrm{G}_{1} \hookrightarrow \mathrm{L} \rightarrow \mathrm{H}$ and $\mathrm{G}_{2} \hookrightarrow \mathrm{H} \rightarrow \mathrm{R}$ gives a (partial) groupoid fibration $G_{1} \hookrightarrow G \rightarrow G_{2}$, where $G$ is the fibre of the composition. $\mathrm{G}_{1} \hookrightarrow \mathrm{L} \rightarrow \mathrm{G}_{2}$ is a (partial) groupoid covering if and only if $\mathrm{G}_{1} \hookrightarrow \mathrm{L} \rightarrow \mathrm{H}$ is so. If $\mathrm{G}_{2} \hookrightarrow \mathrm{H} \rightarrow \mathrm{R}$ is a (partial) groupoid covering then $\mathrm{G}$ and $\mathrm{G}_{1}$ are isomorphic.

We will see that an action of $H$ on $G$ may be transformed along a Morita equivalence $\mathrm{G} \sim \mathrm{K}$ to an action of $\mathrm{H}$ on $\mathrm{K}$. We have an action of $\mathrm{H}$ on $\mathrm{G}$, that is, there is a groupoid $L$ and a groupoid fibration $F: L \rightarrow H$ with fibre $G$. If we have a bibundle equivalence $\mathbb{X}$ from $\mathrm{G}$ to $\mathrm{K}$, then we can construct a generalised action of the groupoid $\mathrm{H}$ on the groupoid $\mathrm{K}$. In particular, we can construct a groupoid $\mathrm{R}$, 
equivalent to the groupoid $L$ by some $\mathbb{Y}$, and a groupoid fibration $E: R \rightarrow H$ with fibre K. Symbolically,

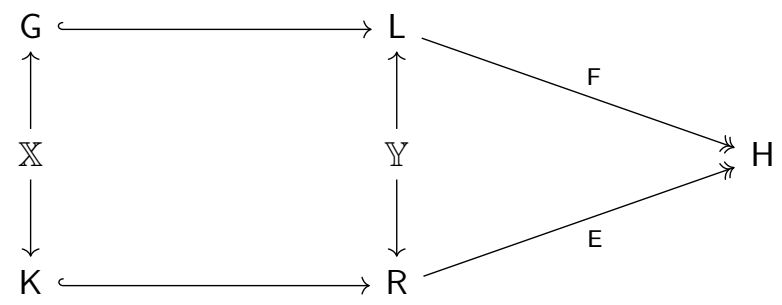

We use the technique of composing bibundle actors. For instance, the arrow space of the groupoid $\mathrm{R}$ is the underlying space of the composition $\mathbb{X} \circ \mathbb{L}^{1} \circ \mathbb{X}^{-1}$ and the bibundle equivalence $\mathbb{Y}$ is constructed by $\mathbb{L}^{1} \circ \mathbb{X}^{-1}$.

We say that the groupoid $G$ is basic if the canonical action of $G$ on its objects is a part of a principal bundle. There is an interesing case when the groupoid fibration has a basic fibre. We define the quotient groupoid $L / G$ for such a groupoid fibration $\mathrm{F}: \mathrm{L} \rightarrow \mathrm{H}$ with basic fibre $\mathrm{G}$. We have a commuting triangle

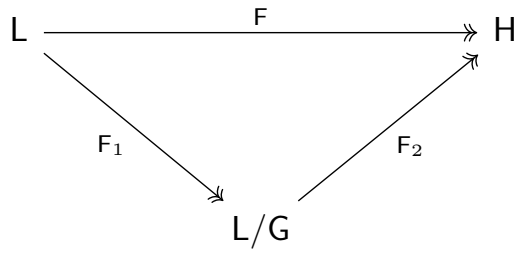

of groupoid fibrations, where $F_{1}: L \rightarrow L / G$ is a cover on objects and $F_{2}: L / G \rightarrow H$ is a groupoid covering. We construct an action of $\mathrm{H}$ on the orbit space of the canonical action of $\mathrm{G}$ on its objects. The quotient groupoid $\mathrm{L} / \mathrm{G}$ is the transformation groupoid of this action. Roughly speaking, under Assumption 5.63 about actions of basic groupoids, the quotient groupoid preserves the property of a groupoid $\mathrm{H}$ to be basic and transmits it to $L$. In a groupoid fibration $G \hookrightarrow L \rightarrow H$, if the groupoids $G$ and $\mathrm{H}$ are basic then so is $\mathrm{L}$.

Partial groupoid fibrations appear in the theory of partial dynamical systems. There is a important notion of a partial action of a group. A partial action of the topological group $\mathrm{G}$ on the topological space $\mathrm{X}$ is a pair $\left(\left\{\mathrm{X}_{g}\right\}_{g \in \mathrm{G}} ;\left\{\theta_{g}\right\}_{g \in \mathrm{G}}\right)$ of open subsets $\left\{\mathrm{X}_{g}\right\}_{g \in \mathrm{G}}$ of $\mathrm{X}$ and homeomorphisms $\left\{\theta_{g}\right\}_{g \in \mathrm{G}}: \mathrm{X}_{g^{-1}} \rightarrow \mathrm{X}_{g}$ such that:

(1) $\mathrm{X}_{e}=\mathrm{X}$ and $\theta_{e}=\mathrm{idx}$;

(2) $\theta_{g}\left(\mathrm{X}_{g^{-1}} \cap \mathrm{X}_{g_{1}}\right)=\mathrm{X}_{g} \cap \mathrm{X}_{g \cdot g_{1}}$;

(3) $\theta_{g}\left(\theta_{g_{1}}(x)\right)=\theta_{g \cdot g_{1}}(x)$, for all $x$ in $\mathrm{X}_{g_{1}^{-1}} \cap \mathrm{X}_{g_{1}^{-1} \cdot g^{-1}}$;

(4) $D^{-1}:=\left\{(g ; x) \in \mathbf{G} \times \mathbf{X} \mid x \in \mathbf{X}_{g^{-1}}\right\}$ is open in $\mathbf{G} \times \mathbf{X}$;

(5) The map $D^{-1} \rightarrow \mathrm{X}$ given by $(g ; x) \mapsto x$ is continuous.

Thus a partial action of $\mathbf{G}$ on $\mathbf{X}$ is also a map from $\mathrm{G}$ into the power set of $\mathrm{X}$, just as for actions of inverse semigroups. For this data we construct a topological groupoid $\mathfrak{G}$ with objects $\mathrm{X}$ and with

$$
\operatorname{Hom}_{\mathfrak{G}}\left(x, x_{1}\right)=\left\{\left(x_{1} ; g ; x\right) \in \mathbf{X} \times \mathbf{G} \times \mathbf{X} \mid(g ; x) \in D^{-1} ; x_{1}=\theta_{g}(x)\right\} .
$$

The multiplication map is given by

$\operatorname{Hom}_{\mathfrak{G}}\left(x, x_{1}\right) \times \operatorname{Hom}_{\mathfrak{G}}\left(x_{2}, x\right) \rightarrow \operatorname{Hom}_{\mathfrak{G}}\left(x_{2}, x_{1}\right),\left(x_{2}, g, x\right) \cdot\left(x, g_{1}, x_{1}\right) \mapsto\left(x_{2}, g \cdot g_{1}, x_{1}\right)$.

It can be shown easily that $\mathfrak{G}$ is a topological groupoid. There is a continuous functor $\mathrm{F}: \mathfrak{G} \rightarrow \mathrm{G}$ given by $\left(x_{1} ; g ; x\right) \mapsto g$, which is a partial groupoid covering from the topological groupoid to the topological group because the continuous map $\mathfrak{G} \rightarrow \mathrm{G} \times \mathrm{X}$ given by $\left(x_{1} ; g ; x\right) \mapsto(g ; x)$ is open and monic. The fibre of this 
partial groupoid covering is the topological space $\mathbf{X}$. Therefore, partial actions of a topological group can be understood as partial groupoid coverings.

There is a connection of this thesis and the thesis of Suliman Albandik [35]. We will see that étale maps form a stronger pretopology in the category of topological spaces. In this category with stronger pretopology a partial bibundle actor is the same as a groupoid correspodence, which are introduced by Suliman Albandik. A partial bibundle actor from a groupoid $\mathrm{G}$ to a groupoid $\mathrm{H}$ is a commuting left action of $\mathrm{G}$ and a right action of $\mathrm{H}$ on the same object $X$, where the right anchor map $\mathrm{sX}_{\mathrm{X}}: \mathrm{X} \rightarrow \mathrm{H}^{0}$ is a partial cover and the right action of $\mathrm{H}$ on $\mathrm{X}$ is basic. A groupoid correspondence from a groupoid $\mathrm{G}$ to a groupoid $\mathrm{H}$ is a commuting left action of $G$ and a right action of $H$ on the same object $X$, where the right anchor map $\mathrm{s}_{\mathrm{X}}: \mathrm{X} \rightarrow \mathrm{H}^{0}$ is étale and the right action of $\mathrm{H}$ on $\mathrm{X}$ is free and proper. In the category of topological spaces with étale maps as partial covers an action of a topological groupoid on a topological space is free and proper if and only if it is basic and the orbit space is Hausdorff. In the case of locally compact, Hausdorff groupoids the groupoid correspondences and bibundle actors are just the same because for this case an action is basic if and only if it is free and proper. We will see that like the groupoid correspondences, there is a bicategory of groupoids with bibundle actors as arrows and equivariant maps as 2-arrows.

\section{Pretopologies}

A stronger pretopology is an extra structure in a general category that allows to develop various kinds of mathematical objects. A category with stronger pretopology is equipped with a notion of "partial cover".

Definition 2.1. Let $\mathcal{C}$ be any category. We say there is defined a stronger pretopology on $\mathcal{C}$ if we have a collection $\mathcal{F}_{p}$ of arrows, called partial covers, with the following properties:

(1) isomorphisms are partial covers;

(2) the composite of two partial covers is a partial cover;

(3) if $x: \mathrm{X} \rightarrow B$ is an arrow in $\mathcal{C}$ and $f: A \rightarrow B$ is a partial cover, then the fibre product $A \times_{f, B, x} \mathrm{X}$ exists in $\mathcal{C}$ and the coordinate projection $\mathrm{pr}_{2}: A \times_{f, B, x} \mathrm{X} \rightarrow \mathrm{X}$ is a partial cover. Symbolically,

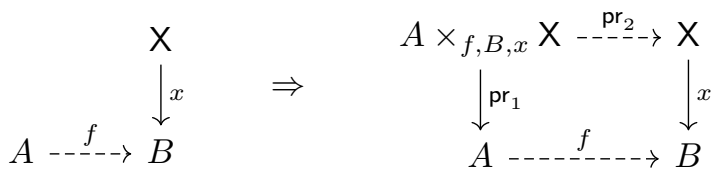

Definition 2.2. Consider a collection $\mathcal{F}$ of such partial covers $f: A-\rightarrow B$ that are the coequalisers of the coordinate projections $\mathrm{pr}_{1}, \mathrm{pr}_{2}: A \times_{f, B, f} A \rightrightarrows A$. Call them covers.

We use dashed arrows $-\rightarrow$ to denote partial covers and double-headed arrows $\rightarrow$ to denote covers.

Remark 2.3. Let $\left(\mathcal{C}, \mathcal{F}_{p}\right)$ be a category with partial covers. If $\mathcal{F}=\mathcal{F}_{p}$ then $(\mathcal{C}, \mathcal{F})$ is a category with a subcanonical pretopology $\mathcal{F}$, as in [47, Definition 2.1].

Remark 2.4. We cannot say that any category $\left(\mathcal{C}, \mathcal{F}_{p}\right)$ with partial covers is a category $(\mathcal{C}, \mathcal{F})$ with pretopology because, in general, the composition of two covers is not a cover.

The following lemmas hold in any category $\left(\mathcal{C}, \mathcal{F}_{p}\right)$ with partial covers.

Lemma 2.5. If a partial cover splits, then it is a cover. 
Proof. Let $f: A \rightarrow B$ be a partial cover and let $g: B \rightarrow A$ be such that $f \circ g=\operatorname{id}_{B}$. We have to show that $f: A \rightarrow B$ is the coequaliser of the coordinate projections $\mathrm{pr}_{1}, \mathrm{pr}_{2}: A \times_{f, B, f} A \rightrightarrows A$.

Let $x: A \rightarrow \mathrm{X}$ be any arrow in $\mathcal{C}$ such that $x \circ \mathrm{pr}_{1}=x \circ \mathrm{pr}_{2}$. We know that $f \circ$ $(g \circ f)=f \circ \operatorname{id}_{A}$. Therefore, there is an arrow $\left(\operatorname{id}_{A} ; g \circ f\right): A \rightarrow A \times_{f, B, f} A$ such that $\mathrm{pr}_{1} \circ\left(\operatorname{id}_{A} ; g \circ f\right)=\operatorname{id}_{A}$ and $\operatorname{pr}_{2} \circ\left(\operatorname{id}_{A} ; g \circ f\right)=g \circ f$. After this we can see that the composition $x \circ g: B \rightarrow \mathrm{X}$ provides a factorization of $x$ through $f$ :

$$
\begin{aligned}
(x \circ g) \circ f & =x \circ(g \circ f) \\
& =x \circ\left(\operatorname{pr}_{2} \circ\left(\operatorname{id}_{A} ; g \circ f\right)\right) \\
& =\left(x \circ \mathrm{pr}_{2}\right) \circ\left(\operatorname{id}_{A} ; g \circ f\right) \\
& =\left(x \circ \operatorname{pr}_{1}\right) \circ\left(\operatorname{id}_{A} ; g \circ f\right) \\
& =x \circ\left(\operatorname{pr}_{1} \circ\left(\operatorname{id}_{A} ; g \circ f\right)\right) \\
& =x \circ i_{A} \\
& =x .
\end{aligned}
$$

Such a factorization is unique because if for some parallel arrows $e_{1}, e_{2}: B \rightrightarrows \mathbf{X}$ we have $e_{1} \circ f=e_{2} \circ f$ then

$$
\begin{aligned}
e_{1} & =e_{1} \circ \mathrm{id}_{B} \\
& =e_{1} \circ(f \circ g) \\
& =\left(e_{1} \circ f\right) \circ g \\
& =\left(e_{2} \circ f\right) \circ g \\
& =e_{2} \circ(f \circ g) \\
& =e_{2} \circ \mathrm{id}_{B} \\
& =e_{2} .
\end{aligned}
$$

Corollary 2.6. An isomorphism is a cover.

Proof. Any isomorphism is a partial cover by Definition 2.1 and it splits by its inverse, so Lemma 2.5 works.

Corollary 2.7. Let $f: A \rightarrow B$ be a partial cover. If $f$ splits, then any pull-back of $f$ is a cover. Moreover, it splits too.

Proof. Let $\mathrm{pr}_{2}: A \times{ }_{B} \mathrm{X} \rightarrow \mathrm{X}$ be the pull-back of $f: A \rightarrow B$ along an arrow $x: \mathrm{X} \rightarrow B$. It is a partial cover by Definition 2.1 Let $f: A \rightarrow B$ split by $g: B \rightarrow A$. We know that

$$
\begin{aligned}
f \circ(g \circ x) & =(f \circ g) \circ x \\
& =\operatorname{id}_{B} \circ x \\
& =x \circ \mathrm{id} x .
\end{aligned}
$$

Therefore, there is an arrow $(g \circ x ; \mathrm{id} \mathbf{X}): \mathrm{X} \rightarrow A \times_{f, B, x} \mathrm{X}$ such that

$$
\mathrm{pr}_{2} \circ(g \circ x ; \mathrm{idx})=\mathrm{idx} \text {. }
$$

This means that $\mathrm{pr}_{2}: A \times_{B} \mathrm{X} \rightarrow \mathrm{X}$ splits. Therefore, it is a cover by Lemma 2.5 .

Corollary 2.8. Let $f: \mathbf{X} \rightarrow \mathrm{Y}$ be a partial cover. The pull-back of $f: \mathbf{X} \rightarrow \mathrm{Y}$ along itself is a cover. Moreover, it splits.

Proof. It is clear that the arrow (idx; idX): $\mathrm{X} \rightarrow \mathrm{X} \times_{f, \mathrm{Y}, f} \mathrm{X}$ is well-defined and $\mathrm{pr}_{2} \circ\left(\mathrm{id}_{\mathbf{X}} ; \mathrm{idX}_{\mathrm{X}}\right)=\mathrm{idx}$. So $\mathrm{pr}_{2}$ is a partial cover because it is a pull-back of $f: \mathrm{X} \rightarrow \mathrm{Y}$, and it splits by (idx;idx). Therefore, it is a cover by Lemma 2.5 
Corollary 2.9. The composition of splitting covers is a cover. Moreover, it splits.

Proof. It is clear that the composition of splitting arrows is a splitting arrow, too. Also, the composition of partial covers is a partial cover. So the composition of splitting covers is a splitting partial cover, and, therefore, it is a cover by Lemma 2.5

Lemma 2.10. The composition of an isomorphism and a cover is a cover.

Proof. Let $f: A \rightarrow B$ and $\varphi: B \rightarrow C$ be a cover and an isomorphism, respectively. The composition $\varphi \circ f$ is a partial cover by the definition of a stronger pretopology. Therefore, the object $A \times_{\varphi \circ f, B, \varphi \circ f} A$ exists. The arrows $\left(\mathrm{pr}_{1} ; \mathrm{pr}_{2}\right): A \times_{f, B, f} A \rightarrow$ $A \times_{\varphi \circ f, B, \varphi \circ f} A$ and $\left(\mathrm{pr}_{1} ; \mathrm{pr}_{2}\right): A \times_{\varphi \circ f, B, \varphi \circ f} A \rightarrow A \times_{f, B, f} A$ are well-defined and inverses of each other. Hence the arrow $f: A \rightarrow B$, and, therefore, the composition $\varphi \circ f$, is a coequaliser of both pairs of the coordinate projections. Thus $\varphi \circ f$ is a cover.

Let $f: A \rightarrow B$ be a cover and let $\varphi: C \rightarrow A$ an isomorphism. There is the arrow $\left(\varphi^{-1} \circ \operatorname{pr}_{1} ; \varphi^{-1} \circ \mathrm{pr}_{2}\right): A \times_{f, B, f} A \rightarrow C \times_{f \circ \varphi, B, f \circ \varphi} C$. It is well-defined because $f \circ \varphi \circ \varphi^{-1} \circ \mathrm{pr}_{1}=f \circ \mathrm{pr}_{1}=f \circ \mathrm{pr}_{2}=f \circ \varphi \circ \varphi^{-1} \circ \mathrm{pr}_{2}$. Let the arrow $x: C \rightarrow \mathrm{X}$ be such that $x \circ \mathrm{pr}_{1}=x \circ \mathrm{pr}_{2}$. We have

$$
\begin{aligned}
x \circ \varphi^{-1} \circ \mathrm{pr}_{1} & =x \circ \mathrm{pr}_{1} \circ\left(\varphi^{-1} \circ \mathrm{pr}_{1} ; \varphi^{-1} \circ \mathrm{pr}_{2}\right) \\
& =x \circ \mathrm{pr}_{2} \circ\left(\varphi^{-1} \circ \mathrm{pr}_{1} ; \varphi^{-1} \circ \mathrm{pr}_{2}\right) \\
& =x \circ \varphi^{-1} \circ \mathrm{pr}_{2} .
\end{aligned}
$$

Since $f: A \rightarrow B$ is a coequaliser of $\mathrm{pr}_{1}, \mathrm{pr}_{2}: A \times_{f, B, f} A \rightrightarrows A$, there is a unique arrow $h: B \rightarrow \mathrm{X}$ such that $h \circ f=x \circ \varphi^{-1}$. This equality is equivalent to $h \circ f \circ \varphi=x$. This means that $f \circ \varphi: C \rightarrow B$ is a coequaliser of $\mathrm{pr}_{1}, \mathrm{pr}_{2}: C \times_{f \circ \varphi, B, f \circ \varphi} C \rightrightarrows C$. Therefore, it is a cover.

Lemma 2.11. If a cover is monic then it is an isomorphism.

Proof. Let the arrow $f: A \rightarrow B$ be a cover and monic. By Definition 2.1 $f: A \rightarrow B$ is a coequalizer of the pair of arrows $\mathrm{pr}_{1}, \mathrm{pr}_{2}: A \times_{f, B, f} A \rightrightarrows A$. Since $f \circ \mathrm{pr}_{1}=f \circ \mathrm{pr}_{2}$ and $f$ is monic, $\mathrm{pr}_{1}=\mathrm{pr}_{2}$. Therefore, $\mathrm{id}_{A} \circ \mathrm{pr}_{1}=\mathrm{id}_{A} \circ \mathrm{pr}_{2}$. Hence there is an arrow $g: B \rightarrow A$ such that $g \circ f=\mathrm{id}_{A}$. Also $f \circ \mathrm{pr}_{1}=f \circ \mathrm{pr}_{2}$ and we have two arrows $\operatorname{id}_{B}, f \circ g: B \rightarrow B$ such that $\operatorname{id}_{B} \circ f=f$ and $(f \circ g) \circ f=f \circ(g \circ f)=f \circ \mathrm{id}_{A}=f$. Thus $f \circ g=\mathrm{id}_{B}$. Therefore, $f$ is an isomorphism.

Lemma 2.12. Let $f: A \rightarrow B$ be a partial cover. $f$ is a cover if and only if it is a coequaliser of some pair of parallel arrows $e_{1}, e_{2}: E \rightrightarrows A$.

Proof. One side of the proof is obvious by the definition of a cover. Now, let $f: A \rightarrow B$ be a coequaliser of parallel arrows $e_{1}, e_{2}: E \rightrightarrows A$. There is the arrow $\left(e_{1} ; e_{2}\right): E \rightarrow A \times_{f, B, f} A$. It is well-defined because $f \circ e_{1}=f \circ e_{2}$. Let the arrow $g: A \rightarrow C$ be such that $g \circ \mathrm{pr}_{1}=g \circ \mathrm{pr}_{2}$, where $\mathrm{pr}_{1}$ and $\mathrm{pr}_{2}$ are the coordinate projections of $A \times_{f, B, f} A$. We have $g \circ e_{1}=g \circ \mathrm{pr}_{1} \circ\left(e_{1} ; e_{2}\right)=g \circ \mathrm{pr}_{2} \circ\left(e_{1} ; e_{2}\right)=g \circ e_{2}$. Since $f: A \rightarrow B$ is the coequaliser of $e_{1}, e_{2}: E \rightrightarrows A$, there is a unique $h: B \rightarrow C$ such that $h \circ f=g$. Therefore, $f$ is a coequaliser of the coordinate projections $\mathrm{pr}_{1}, \mathrm{pr}_{2}: A \times_{f, B, f} A \rightrightarrows A$. Hence it is a cover.

Lemma 2.13. Assume that the pull-back of an arrow $g: C \rightarrow B$ along a cover $f: A \rightarrow B$ is an isomorphism. If the coordinate projection $\mathrm{pr}_{2}: A \times_{f, B, g} C \rightarrow C$ is epic, then $g: C \rightarrow B$ is an isomorphism, too. 
Proof. Let $f: A \rightarrow B$ be a coequaliser of the pair of parallel arrows $e_{1}, e_{2}: E \rightrightarrows A$.

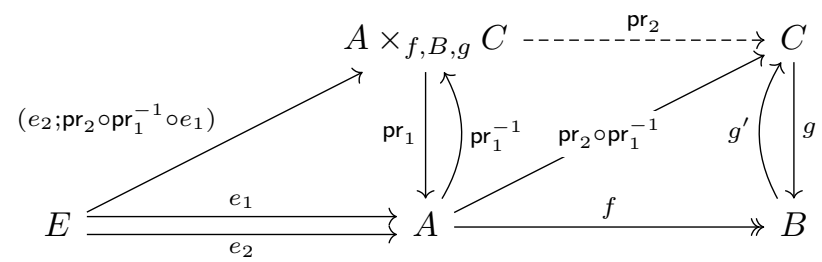

There is an arrow $\left(e_{2} ; \mathrm{pr}_{2} \circ \mathrm{pr}_{1}^{-1} \circ e_{1}\right): E \rightarrow A \times_{f, B, g} C$. It is well-defined becasue

$$
\begin{aligned}
g \circ \mathrm{pr}_{2} \circ \mathrm{pr}_{1}^{-1} \circ e_{1} & =f \circ \mathrm{pr}_{1} \circ \mathrm{pr}_{1}^{-1} \circ e_{1} \\
& =f \circ e_{1} \\
& =f \circ e_{2} .
\end{aligned}
$$

Since $\operatorname{pr}_{1} \circ\left(e_{2} ; \operatorname{pr}_{2} \circ \operatorname{pr}_{1}^{-1} \circ e_{1}\right)=e_{2}$, we have $\operatorname{pr}_{1}^{-1} \circ e_{2}=\left(e_{2} ; \operatorname{pr}_{2} \circ \operatorname{pr}_{1}^{-1} \circ e_{1}\right)$. Therefore, $\mathrm{pr}_{2} \circ \mathrm{pr}_{1}^{-1} \circ e_{2}=\mathrm{pr}_{2} \circ\left(e_{2} ; \mathrm{pr}_{2} \circ \mathrm{pr}_{1}^{-1} \circ e_{1}\right)=\mathrm{pr}_{2} \circ \mathrm{pr}_{1}^{-1} \circ e_{1}$. Hence there is a unique arrow $g^{\prime}: B \rightarrow C$ such that $g^{\prime} \circ f=\mathrm{pr}_{2} \circ \mathrm{pr}_{1}^{-1}$. We have

$$
\begin{aligned}
g \circ g^{\prime} \circ f & =g \circ \mathrm{pr}_{2} \circ \mathrm{pr}_{1}^{-1} \\
& =f \circ \mathrm{pr}_{1} \circ \mathrm{pr}_{1}^{-1} \\
& =f .
\end{aligned}
$$

Since $f: A \rightarrow B$ is a cover, it is epic and, therefore, $g \circ g^{\prime}=\mathrm{id}_{B}$. Also we have

$$
\begin{aligned}
g^{\prime} \circ g \circ \mathrm{pr}_{2} & =g^{\prime} \circ f \circ \mathrm{pr}_{1} \\
& =\mathrm{pr}_{2} \circ \mathrm{pr}_{1}^{-1} \circ \mathrm{pr}_{1} \\
& =\mathrm{pr}_{2} .
\end{aligned}
$$

Since $\mathrm{pr}_{2}: A \times_{f, B, g} C \rightarrow C$ is epic, we have $g^{\prime} \circ g=\mathrm{id}_{C}$. So $g^{\prime}$ is an inverse of $g$, and, therefore, $g$ is an isomorphism.

2.1. Extra assumptions on stronger pretopologies. Let $\left(\mathcal{C}, \mathcal{F}_{p}\right)$ be a category with partial covers. We know that the composition of splitting covers is a cover (Corollary 2.9). Also, the composition of an isomorphism and a cover is a cover (Lemma 2.10p. But, generally, we cannot say that the composition of covers is a cover. The following assumption on the stronger pretopology is about this.

Assumption 2.14. 47, Definition 2.1]. The composition of covers is a cover.

The next assumption is about the pull-back of a cover. We know that the pullback of a splitting cover is a cover (Corollary 2.7). Also, the pull-back of a cover along itself is a cover (Corollary 2.8). But, generally, the pull-back of a cover is not a cover. In some results we require the following assumption.

Assumption 2.15. 47, Definition 2.1]. Any pull-back of a cover is a cover.

Remark 2.16. Under Assumption 2.15 we do not need the requirement that the coordinate projection $\mathrm{pr}_{2}: A \times_{f, B, g} C \rightarrow C$ is epic in Lemma 2.13 because it automatically comes from Assumption $2.15 \mathrm{pr}_{2}: A \times_{f, B, g} C \rightarrow C$ is a pull-back of the cover $f: A \rightarrow B$. Therefore, it is a cover. Thus it is epic.

Remark 2.17. Under Assumptions 2.14 and 2.15, a category $\left(\mathcal{C}, \mathcal{F}_{p}\right)$ with partial covers is a category $(\mathcal{C}, \mathcal{F})$ with pretopology as defined in 47 .

The next assumption is important for principal bundles and arrows between the orbit spaces. We need this assumption for defining a composition of bibundle functors. 
Assumption 2.18. If a pull-back of $f$ along a cover is a cover, then $f$ is a cover, too.

The following assumption is stronger than the previous one. It is necessary when we want to compose bibundle actors.

Assumption 2.19. If $f \circ g$ and $g$ are covers, then so is $f$.

The following assumption is about the final object. We know that the obvious example of a groupoid is a group. A group is a groupoid with only one identity. So we need the following assumption to define groups in a category $\left(\mathcal{C}, \mathcal{F}_{p}\right)$ with partial covers.

Assumption 2.20. There is a final object in $\left(\mathcal{C}, \mathcal{F}_{p}\right)$ and all arrows to it are covers.

Remark 2.21. Under Assumption 2.20 the category $\left(\mathcal{C}, \mathcal{F}_{p}\right)$ has finite products because they are fibre products over the final object.

\section{Groupoids in a CATEgory With partial COVERS}

Before defining a groupoid we need to explain the notion of elementwise expressions. Let $\mathrm{A}$ be any object in $\mathcal{C}$. An element $x$ in $\mathrm{A}$ is interpreted as arrow in $\mathcal{C}$ from some object $? \in \in \mathcal{C}$ to $A$. The elements of $A$ form a category, which determines $A$ by the Yoneda Lemma. If $f: \mathrm{A} \rightarrow \mathrm{B}$ is any arrow in $\mathcal{C}$ then for any element $x$ in $\mathrm{A}$ $f(x)$ is interpreted as the composition $f \circ x: ? \rightarrow \mathrm{B}$, which is an element in $\mathrm{B}$.

Definition 3.1. A groupoid $G=\left(G^{0}, G^{1}, r, s, m\right)$ in the category $\left(\mathcal{C}, \mathcal{F}_{p}\right)$ with partial covers consists of

- objects $\mathrm{G}^{0}, \mathrm{G}^{1} \in \in \mathcal{C}$

- arrows $r: \mathrm{G}^{1} \rightarrow \mathrm{G}^{0}, \quad \mathrm{~s}: \mathrm{G}^{1} \rightarrow \mathrm{G}^{0}$ and $\mathrm{m}: \mathrm{G}^{1} \times{ }_{\mathrm{s}, \mathrm{G}^{0}, \mathrm{r}} \mathrm{G}^{1} \rightarrow \mathrm{G}^{1}$;

such that

(1) $r$ and s are covers;

(2) for the coordinate projections $\mathrm{pr}_{1}, \mathrm{pr}_{2}: \mathrm{G}^{1} \times_{\mathrm{s}, \mathrm{G}^{0}, \mathrm{r}} \mathrm{G}^{1} \rightrightarrows \mathrm{G}^{1}$ we have equations $\mathrm{r} \circ \mathrm{m}=\mathrm{r} \circ \mathrm{pr}_{1} ; \quad \mathrm{s} \circ \mathrm{m}=\mathrm{s} \circ \mathrm{pr}_{2} ; \quad \mathrm{r}\left(g \cdot g_{1}\right)=\mathrm{r}(g) ; \quad \mathrm{s}\left(g \cdot g_{1}\right)=\mathrm{s}\left(g_{1}\right)$, for all $g, g_{1} \in \mathrm{G}^{1}$ with $\mathrm{r}\left(g_{1}\right)=\mathrm{s}(g)$;

(3) $\mathrm{m}$ is associative, that is, the following diagram commutes:

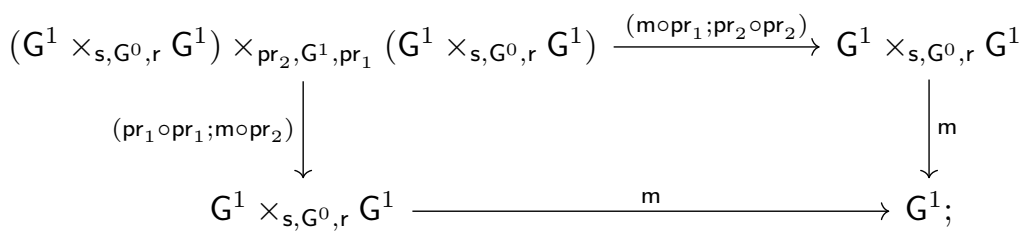

$\mathrm{m} \circ\left(\mathrm{m} \circ \mathrm{pr}_{1} ; \mathrm{pr}_{2} \circ \mathrm{pr}_{2}\right)=\mathrm{m} \circ\left(\mathrm{pr}_{1} \circ \mathrm{pr}_{1} ; \mathrm{m} \circ \mathrm{pr}_{2}\right) ; \quad\left(g \cdot g_{1}\right) \cdot g_{2}=g \cdot\left(g_{1} \cdot g_{2}\right)$, $\forall g, g_{1}, g_{2} \in \mathrm{G}^{1}$ with $\mathrm{s}(g)=\mathrm{r}\left(g_{1}\right)$ and $\mathrm{s}\left(g_{1}\right)=\mathrm{r}\left(g_{2}\right)$;

(4) the maps

$$
\begin{array}{ll}
\left(\mathrm{pr}_{2} ; \mathrm{m}\right): \mathrm{G}^{1} \times_{\mathrm{s}, \mathrm{G}^{0}, \mathrm{r}} \mathrm{G}^{1} \longrightarrow \mathrm{G}^{1} \times_{\mathrm{s}, \mathrm{G}^{0}, \mathrm{~s}} \mathrm{G}^{1}, & \left(g ; g_{1}\right) \mapsto\left(g_{1} ; g \cdot g_{1}\right) ; \\
\left(\mathrm{pr}_{1} ; \mathrm{m}\right): \mathrm{G}^{1} \times_{\mathrm{s}, \mathrm{G}^{0}, \mathrm{r}} \mathrm{G}^{1} \longrightarrow \mathrm{G}^{1} \times_{\mathrm{r}, \mathrm{G}^{0}, \mathrm{r}} \mathrm{G}^{1}, & \left(g ; g_{1}\right) \mapsto\left(g ; g \cdot g_{1}\right) ;
\end{array}
$$

are isomorphisms;

We call the objects $\mathrm{G}^{0}, \mathrm{G}^{1}$ and $\mathrm{G}^{1} \times_{\mathrm{s}, \mathrm{G}^{0}, \mathrm{r}} \mathrm{G}^{1}$ the objects, arrows and composable pairs, and the arrows $\mathrm{r}, \mathrm{s}$ and $\mathrm{m}$ the range, source and multiplication maps, respectively.

Here, for $g, g_{1} \in \mathrm{G}^{1}$ with $\mathrm{s}(g)=\mathrm{r}\left(g_{1}\right), g \cdot g_{1}$ means the composite arrow

$$
? \stackrel{\left(g ; g_{1}\right)}{\longrightarrow} \mathrm{G}^{1} \times_{\mathrm{s}, \mathrm{G}^{0}, \mathrm{r}} \mathrm{G}^{1} \stackrel{\mathrm{m}}{\rightarrow} \mathrm{G}^{1} \text {. }
$$


Remark 3.2. All objects in (2) (3) and (4) exist because of (1) The arrows $\left(\mathrm{m} \circ \mathrm{pr}_{1} ; \mathrm{pr}_{2} \circ \mathrm{pr}_{2}\right),\left(\mathrm{pr}_{1} \circ \mathrm{pr}_{1} ; \mathrm{m} \circ \mathrm{pr}_{2}\right),\left(\mathrm{pr}_{2}, \mathrm{~m}\right)$ and $\left(\mathrm{pr}_{1}, \mathrm{~m}\right)$ are well-defined because of the equalities in (2)

Remark 3.3. Definition 3.1 is the same as Definition 3.4 in 47]. The only difference is the structure of the category in which we define groupoids.

Lemma 3.4. Let $\mathrm{G}=\left(\mathrm{G}^{0}, \mathrm{G}^{1}, \mathrm{r}, \mathrm{s}, \mathrm{m}\right)$ be a groupoid. Then the coordinate projections $\mathrm{pr}_{1}, \mathrm{pr}_{2}: \mathrm{G}^{1} \times_{\mathrm{s}, \mathrm{G}^{0}, \mathrm{r}} \mathrm{G}^{1} \rightrightarrows \mathrm{G}^{1}$ are covers. Moreover, they split.

Proof. It is clear that the arrow $\left(\mathrm{id}_{\mathrm{G}^{1}} ; \mathrm{id}_{\mathrm{G}^{1}}\right): \mathrm{G}^{1} \rightarrow \mathrm{G}^{1} \times_{\mathrm{s}, \mathrm{G}^{0}, \mathrm{~s}} \mathrm{G}^{1}$ is well-defined. We also have $\mathrm{pr}_{2} \circ\left(\mathrm{pr}_{2} ; \mathrm{m}\right)^{-1} \circ\left(\mathrm{id}_{\mathrm{G}^{1}} ; \mathrm{id}_{\mathrm{G}^{1}}\right)=\mathrm{pr}_{2} \circ\left(\mathrm{id}_{\mathrm{G}^{1}} ; \mathrm{id}_{\mathrm{G}^{1}}\right)=\mathrm{id}_{\mathrm{G}^{1}}$. So $\mathrm{pr}_{2}$ splits by $\left(\mathrm{pr}_{2} ; \mathrm{m}\right)^{-1} \circ\left(\mathrm{id}_{\mathrm{G}^{1}} ; \mathrm{id}_{\mathrm{G}^{1}}\right)$. Analogously, $\mathrm{pr}_{1}$ splits by the arrow $\left(\mathrm{pr}_{1} ; \mathrm{m}\right)^{-1} \circ\left(\mathrm{id}_{\mathrm{G}^{1}} ; \mathrm{id}_{\mathrm{G}^{1}}\right)$. The coordinate projections are partial covers because they are pull-backs of the source and range maps. Therefore, they are covers by Lemma 2.5 .

Lemma 3.5. Let $\mathrm{G}=\left(\mathrm{G}^{0}, \mathrm{G}^{1}, \mathrm{r}, \mathrm{s}, \mathrm{m}\right)$ be a groupoid and let $\mathrm{X}$ be an object in $\mathcal{C}$. Let $x_{1}, x_{2}, g \in \mathcal{C}\left(\mathrm{X}, \mathrm{G}^{1}\right)$ be such that $\left(x_{1} ; g\right),\left(x_{2} ; g\right) \in \mathcal{C}\left(\mathrm{X}, \mathrm{G}^{1} \times_{\mathrm{s}, \mathrm{G}^{0}, \mathrm{r}} \mathrm{G}^{1}\right)$ are well-defined arrows and $\mathrm{m} \circ\left(x_{1} ; g\right)=\mathrm{m} \circ\left(x_{2} ; g\right)$, then $x_{1}=x_{2}$.

Proof. Consider the following composition:

$$
\mathrm{X} \stackrel{\left(x_{1} ; g\right)}{\longrightarrow} \mathrm{G}^{1} \times_{\mathrm{s}, \mathrm{G}^{0}, \mathrm{r}} \mathrm{G}^{1} \stackrel{\left(\mathrm{pr}_{2} ; \mathrm{m}\right)}{\longrightarrow} \mathrm{G}^{1} \times_{\mathrm{s}, \mathrm{G}^{0}, \mathrm{~s}} \mathrm{G}^{1} .
$$

We have

$$
\begin{aligned}
\left(\mathrm{pr}_{2} ; \mathrm{m}\right) \circ\left(x_{1} ; g\right) & =\left(\mathrm{pr}_{2} \circ\left(x_{1} ; g\right) ; \mathrm{m} \circ\left(x_{1} ; g\right)\right) \\
& =\left(g ; \mathrm{m} \circ\left(x_{1} ; g\right)\right) .
\end{aligned}
$$

Analogously,

Therefore,

$$
\left(\mathrm{pr}_{2} ; \mathrm{m}\right) \circ\left(x_{2} ; g\right)=\left(g ; \mathrm{m} \circ\left(x_{2} ; g\right)\right)
$$

$$
\left(\mathrm{pr}_{2} ; \mathrm{m}\right) \circ\left(x_{1} ; g\right)=\left(\mathrm{pr}_{2} ; \mathrm{m}\right) \circ\left(x_{2} ; g\right) .
$$

The arrow $\left(\mathrm{pr}_{2} ; \mathrm{m}\right)$ is an isomorphism by Definition 3.1. So $\left(x_{1} ; g\right)=\left(x_{2} ; g\right)$. Hence $x_{1}=x_{2}$.

Remark 3.6. We can also deduce that $x_{1}=x_{2}$ if the arrows $\left(g ; x_{1}\right)$ and $\left(g ; x_{2}\right)$ are well-defined and $\mathrm{m} \circ\left(g ; x_{1}\right)=\mathrm{m} \circ\left(g ; x_{2}\right)$. In this case, we use the isomorphism $\left(\mathrm{m} ; \mathrm{pr}_{1}\right)$.

Remark 3.7. In elementwise notation, Lemma 3.5 and Remark 3.6 say the following: If $x_{1} \cdot g=x_{2} \cdot g$ then $x_{1}=x_{2}$, and if $g \cdot x_{1}=g \cdot x_{2}$ then $x_{1}=x_{2}$.

Proposition 3.8. Let $\mathrm{G}=\left(\mathrm{G}^{0}, \mathrm{G}^{1}, \mathrm{r}, \mathrm{s}, \mathrm{m}\right)$ be a groupoid. There are arrows $\mathrm{u}: \mathrm{G}^{0} \rightarrow \mathrm{G}^{1}$ and $\mathrm{i}: \mathrm{G}^{1} \rightarrow \mathrm{G}^{1}$ such that the following equalities hold:

(1) $\mathrm{r} \circ \mathrm{u}=\mathrm{id}_{\mathrm{G}^{0}}=\mathrm{s} \circ \mathrm{u} ; \quad \mathrm{r}\left(1_{g_{0}}\right)=g_{0}=\mathrm{r}\left(1_{g_{0}}\right), \forall g_{0} \in \mathrm{G}^{0}$;

(2) $\mathrm{m} \circ\left(\mathrm{u} \circ \mathrm{r} ; \mathrm{id}_{\mathrm{G}^{1}}\right)=\mathrm{id}_{\mathrm{G}^{1}}=\mathrm{m} \circ\left(\mathrm{id}_{\mathrm{G}^{1}} ; \mathrm{u} \circ \mathrm{s}\right) ; \quad 1_{\mathrm{r}(g)} \cdot g=g=g \cdot 1_{\mathrm{s}(g)}, \forall g \in \mathrm{G}^{1}$;

(3) $\mathrm{s} \circ \mathrm{i}=\mathrm{r} ; \mathrm{r} \circ \mathrm{i}=\mathrm{s} ; \quad \mathrm{s}\left(g^{-1}\right)=\mathrm{r}(g) ; \mathrm{r}\left(g^{-1}\right)=\mathrm{s}(g), \forall g \in \mathrm{G}^{1}$;

(4) $\mathrm{m} \circ\left(\mathrm{i} ; \mathrm{id}_{\mathrm{G}^{1}}\right)=$ uos; $\mathrm{m} \circ\left(\mathrm{id}_{\mathrm{G}^{1}} ; \mathrm{i}\right)=\mathrm{u} \circ \mathrm{r} ; \quad g^{-1} \cdot g=1_{\mathrm{s}(g)} ; g \cdot g^{-1}=1_{\mathrm{r}(g)}, \forall g \in \mathrm{G}^{1}$;

Here, for $g_{0} \in \mathrm{G}^{0}, 1_{g_{0}}$ means the composite arrow $\mathrm{u} \circ g_{0}$, and for $g \in \mathrm{G}^{1}, g^{-1}$ means i $\circ g$.

Proof. First we construct the arrow u : $\mathrm{G}^{0} \rightarrow \mathrm{G}^{1}$. Consider the following composition:

$$
\text { ũ: } G^{1} \stackrel{\left(\mathrm{id}_{\mathrm{G}^{1}} ; \mathrm{id}_{\mathrm{G}^{1}}\right)}{\longrightarrow} \mathrm{G}^{1} \times_{s, \mathrm{G}^{0}, s} \mathrm{G}^{1} \stackrel{\left(\mathrm{pr}_{2} ; \mathrm{m}\right)^{-1}}{\longrightarrow} \mathrm{G}^{1} \times_{s, \mathrm{G}^{0}, \mathrm{r}} \mathrm{G}^{1} \stackrel{\mathrm{pr}_{1}}{\longrightarrow} \mathrm{G}^{1} \text {. }
$$

We have $\left(\mathrm{pr}_{2} ; \mathrm{m}\right)^{-1}(g ; g)=(\tilde{\mathrm{u}}(g) ; g)$. Hence $(\tilde{\mathrm{u}}(g) ; g)$ is a composable pair and $\left(\mathrm{pr}_{2} ; \mathrm{m}\right)(\tilde{\mathrm{u}}(g) ; g)=(g ; g)$. Thus $\tilde{\mathrm{u}}(g) \cdot g=g$ for all $g \in \mathrm{G}^{1}$. So we have

$$
\mathrm{m} \circ\left(\tilde{\mathrm{u}} ; \mathrm{id}_{\mathrm{G}^{1}}\right)=\mathrm{id}_{\mathrm{G}^{1}} .
$$


Consider any composable pair $\left(g ; g_{1}\right)$ of arrows in $\mathrm{G}^{1}$. We have $\tilde{\mathrm{u}}\left(g \cdot g_{1}\right) \cdot g \cdot g_{1}=g \cdot g_{1}$. Thus $\tilde{\mathbf{u}}\left(g \cdot g_{1}\right) \cdot g=g$ by Lemma 3.5 We also have $\tilde{\mathbf{u}}(g) \cdot g=g$. Hence $\tilde{\mathbf{u}}\left(g \cdot g_{1}\right)=\tilde{\mathbf{u}}(g)$. Since the arrow $\left(\mathrm{pr}_{2} ; \mathrm{m}\right)$ is an isomorphism, we have

$$
\tilde{\mathrm{u}}(g)=\tilde{\mathrm{u}}\left(g^{\prime}\right),
$$

for all $g, g^{\prime} \in \mathrm{G}^{1}$ with $\mathrm{r}(g)=\mathrm{r}\left(g^{\prime}\right)$.

The range map is a cover, hence it is a coequaliser of the coordinate projections $\mathrm{pr}_{1}, \mathrm{pr}_{2}: \mathrm{G}^{1} \times_{\mathrm{r}, \mathrm{G}^{0}, \mathrm{r}} \mathrm{G}^{1} \rightrightarrows \mathrm{G}^{1}$. Since $\mathrm{pr}_{1} \circ \tilde{\mathrm{u}}=\mathrm{pr}_{2} \circ \tilde{\mathrm{u}}$, there is a unique arrow $\mathrm{u}: \mathrm{G}^{0} \rightarrow \mathrm{G}^{1}$ such that $\mathrm{u} \circ \mathrm{r}=\tilde{u}$. Denote the element $\mathrm{u}\left(g_{0}\right)$ by $1_{g_{0}}$ for all $g_{0} \in \mathrm{G}^{0}$. So we have $1_{r(g)}=\tilde{u}(g)$ for all $g \in \mathrm{G}^{1}$. The construction of the arrow $\mathrm{u}: \mathrm{G}^{0} \rightarrow \mathrm{G}^{1}$ is done.

The next step is to show the properties in (1) and (2). We proved above that $\mathrm{s} \circ \tilde{\mathrm{u}}=\mathrm{r}$. Thus $\mathrm{s} \circ \mathrm{u} \circ \mathrm{r}=\mathrm{r}$. Thus $\mathrm{s} \circ \mathrm{u}=\mathrm{id}_{\mathrm{G}^{0}}$ because the range map is epic. We also have $r(\tilde{\mathbf{u}}(g))=r(\tilde{\mathbf{u}}(g) \cdot g)=r(g)$ for all $g \in \mathrm{G}^{1}$. Therefore, $r=r \circ \tilde{u}=r \circ \mathbf{u} \circ \mathbf{r}$, hence $r \circ u=i d_{\mathrm{G}^{0}}$. So the proof of (1) is done.

The first part of (2) follows from $\mathrm{m} \circ\left(\tilde{\mathrm{u}} ; \mathrm{id}_{\mathrm{G}^{1}}\right)=\mathrm{id}_{\mathrm{G}^{1}}$ and $\mathrm{u} \circ \mathrm{r}=\tilde{\mathrm{u}}$, which are proved above. For proving the second part consider a composable pair $\left(g ; g_{1}\right)$ of arrows in $\mathrm{G}^{1}$. Since $\mathrm{s}\left(1_{\mathbf{s}(g)}\right)=\mathbf{s}(g)=\mathrm{r}\left(g_{1}\right)$, the pair $\left(1_{\mathbf{s}(g)} ; g_{1}\right)$ is composable, too. We have $g \cdot 1_{\mathrm{s}(g)} \cdot g_{1}=g \cdot 1_{\mathrm{r}\left(g_{1}\right)} \cdot g_{1}=g \cdot g_{1}$. Thus $g \cdot 1_{\mathrm{s}(g)}=g$ by Lemma 3.5. That is, $\mathrm{m} \circ\left(\mathrm{id}_{\mathrm{G}^{1}} ; \mathrm{u} \circ \mathrm{s}\right)=\mathrm{id}_{\mathrm{G}^{1}}$. So (2) is completely proved.

Now we have to construct the arrow $\mathrm{i}: \mathrm{G}^{1} \rightarrow \mathrm{G}^{1}$. We can directly name this arrow. Let $\mathrm{i}: \mathrm{G}^{1} \rightarrow \mathrm{G}^{1}$ be the following composition:

$$
\mathrm{G}^{1} \stackrel{\left(\mathrm{id}_{\mathrm{G}^{1}} ; \mathrm{uos}\right)}{\longrightarrow} \mathrm{G}^{1} \times_{\mathrm{s}, \mathrm{G}^{0}, \mathrm{~s}} \mathrm{G}^{1} \stackrel{\left(\mathrm{pr}_{2} ; \mathrm{m}\right)^{-1}}{\longrightarrow} \mathrm{G}^{1} \times_{\mathrm{s}, \mathrm{G}^{0}, \mathrm{r}} \mathrm{G}^{1} \stackrel{\mathrm{pr}_{1}}{\longrightarrow} \mathrm{G}^{1} \text {. }
$$

The first arrow is well-defined because $\mathrm{s}\left(1_{\mathrm{s}(g)}\right)=\mathrm{s}(g)$. Denote the element $\mathrm{i}(g)$ by $g^{-1}$. We have $\left(\mathrm{pr}_{2} ; \mathrm{m}\right)^{-1}\left(g ; 1_{\mathrm{s}(g)}\right)=\left(g^{-1} ; g\right)$. Hence $\left(g^{-1} ; g\right)$ is a composable pair, that is, $\mathrm{s}\left(g^{-1}\right)=\mathrm{r}(g)$, and $\left(\mathrm{pr}_{2} ; \mathrm{m}\right)\left(g^{-1} ; g\right)=\left(g ; 1_{\mathrm{s}(g)}\right)$. Thus $g^{-1} \cdot g=1_{\mathrm{s}(g)}$, for all $g \in \mathrm{G}^{1}$. So $\mathrm{s} \circ \mathrm{i}=\mathrm{r}$ and $\mathrm{m} \circ\left(\mathrm{i} ; \mathrm{id}_{\mathrm{G}^{1}}\right)=\mathrm{u} \circ \mathrm{s}$. We also have $\mathrm{r}\left(g^{-1}\right)=\mathrm{r}\left(g^{-1} \cdot g\right)=\mathrm{r}\left(1_{\mathrm{s}(g)}\right)=\mathrm{s}(g)$, for all $g \in \mathrm{G}^{1}$. This means that $\mathrm{r} \circ \mathrm{i}=\mathrm{s}$. Hence the pair $\left(g ; g^{-1}\right)$ is composable. We have

$$
\begin{aligned}
\left(g \cdot g^{-1}\right) \cdot g & =g \cdot\left(g^{-1} \cdot g\right) \\
& =g \cdot 1_{\mathrm{s}(g)} \\
& =g \\
& =1_{\mathrm{r}(g)} \cdot g .
\end{aligned}
$$

Therefore, $g \cdot g^{-1}=1_{\mathrm{r}(g)}$, for all $g \in \mathrm{G}^{1}$ by Lemma 3.5 . Hence $\mathrm{m} \circ\left(\mathrm{id}_{\mathrm{G}^{1}} ; \mathrm{i}\right)=\mathrm{u} \circ \mathrm{r}$ and the proof is done.

We call the arrows $\mathrm{u}: \mathrm{G}^{0} \rightarrow \mathrm{G}^{1}$ and $\mathrm{i}: \mathrm{G}^{1} \rightarrow \mathrm{G}^{1}$ described in Lemma 3.8 the unit and inverse maps of the groupoid $G=\left(G^{0}, G^{1}, r, s, m\right)$, respectively. If the arrows $\mathrm{u}: \mathrm{G}^{0} \rightarrow \mathrm{G}^{1}$ and $\mathrm{i}: \mathrm{G}^{1} \rightarrow \mathrm{G}^{1}$ are the unit and inverse maps of the groupoid $\mathrm{G}=\left(\mathrm{G}^{0}, \mathrm{G}^{1}, \mathrm{r}, \mathrm{s}, \mathrm{m}\right)$ we shortly say it is a groupoid $\mathrm{G}=\left(\mathrm{G}^{0}, \mathrm{G}^{1}, \mathrm{r}, \mathrm{s}, \mathrm{m}, \mathrm{u}, \mathrm{i}\right)$.

Corollary 3.9. Let $\mathrm{G}=\left(\mathrm{G}^{0}, \mathrm{G}^{1}, \mathrm{r}, \mathrm{s}, \mathrm{m}, \mathrm{u}, \mathrm{i}\right)$ be a groupoid. then

(1) $\mathrm{m} \circ(\mathrm{u} ; \mathrm{u})=\mathrm{u}, \quad 1_{g_{0}} \cdot 1_{g_{0}}=1_{g_{0}}, \forall g_{0} \in \mathrm{G}^{0}$;

(2) $\mathrm{i} \circ \mathrm{i}=\mathrm{id}_{\mathrm{G}^{1}}, \quad\left(g^{-1}\right)^{-1}=g, \forall g \in \mathrm{G}^{1}$;

(3) $\mathrm{i} \circ \mathrm{u}=\mathrm{u}, \quad\left(1_{g_{0}}\right)^{-1}=1_{g_{0}}, \forall g_{0} \in \mathrm{G}^{0}$;

(4) $\mathrm{m} \circ\left(\mathrm{i} \circ \mathrm{pr}_{1} ; \mathrm{i} \circ \mathrm{pr}_{2}\right)=\mathrm{i} \circ \mathrm{m} \circ\left(\mathrm{pr}_{2} ; \mathrm{pr}_{1}\right), \quad g_{1}^{-1} \cdot g^{-1}=\left(g \cdot g_{1}\right)^{-1}$, for all $g, g_{1} \in \mathrm{G}^{1}$ with $\mathrm{s}(g)=\mathrm{r}\left(g_{1}\right)$. Here $\mathrm{pr}_{1}$ and $\mathrm{pr}_{2}$ are the coordinate projections of $\mathrm{G}^{1} \times_{\mathrm{r}, \mathrm{G}^{0}, \mathrm{~s}} \mathrm{G}^{1}$.

Proof. We already know that $1_{\mathrm{r}(g)} \cdot g=g$, for all $g \in \mathrm{G}^{1}$ and $\mathrm{r}\left(1_{g_{0}}\right)=g_{0}$ for all $g_{0} \in \mathrm{G}^{0}$. Hence $1_{g_{0}} \cdot 1_{g_{0}}=1_{\mathrm{r}\left(1_{g_{0}}\right)} \cdot 1_{g_{0}}=1_{g_{0}}$, for all $g_{0} \in \mathrm{G}^{0} \cdot(1)$ is proved. We also 
have

$$
\begin{aligned}
\left(g^{-1}\right)^{-1} \cdot g^{-1} & =1_{\mathrm{s}\left(g^{-1}\right)} \\
& =1_{\mathrm{r}(g)} \\
& =g \cdot g^{-1},
\end{aligned}
$$

for all $g \in \mathrm{G}^{1}$. Hence $\left(g^{-1}\right)^{-1}=g$, for all $g \in \mathrm{G}^{1}$ by Lemma 3.5. (2) is done. Also

$$
\begin{aligned}
\left(1_{g_{0}}\right)^{-1} \cdot 1_{g_{0}} & =1_{\mathrm{s}\left(1_{g_{0}}\right)} \\
& =1_{g_{0}} \\
& =1_{g_{0}} \cdot 1_{g_{0}} .
\end{aligned}
$$

Therefore, $\left(1_{g_{0}}\right)^{-1}=1_{g_{0}}$, for all $g_{0} \in \mathrm{G}^{0}$. Hence (3) holds. Consider any composable pair $\left(g ; g_{1}\right)$ of arrows in $\mathrm{G}^{1}$. We have

$$
\begin{aligned}
\left(g \cdot g_{1}\right)^{-1} \cdot\left(g \cdot g_{1}\right) & =1_{\mathrm{s}\left(g \cdot g_{1}\right)} \\
& =1_{\mathrm{s}\left(g_{1}\right)} \\
& =g_{1}^{-1} \cdot g_{1} \\
& =g_{1}^{-1} \cdot 1_{\mathrm{r}\left(g_{1}\right)} \cdot g_{1} \\
& =g_{1}^{-1} \cdot 1_{\mathrm{s}(g)} \cdot g_{1} \\
& =g_{1}^{-1} \cdot g^{-1} \cdot g \cdot g_{1} \\
& =\left(g_{1}^{-1} \cdot g^{-1}\right) \cdot\left(g \cdot g_{1}\right) .
\end{aligned}
$$

Therefore, $g_{1}^{-1} \cdot g^{-1}=\left(g \cdot g_{1}\right)^{-1}$, for all $g, g_{1} \in \mathrm{G}^{1}$ with $\mathrm{s}(g)=\mathrm{r}\left(g_{1}\right)$. So the proof is done.

Lemma 3.10. If $\mathrm{G}=\left(\mathrm{G}^{0}, \mathrm{G}^{1}, \mathrm{r}, \mathrm{s}, \mathrm{m}, \mathrm{u}, \mathrm{i}\right)$ is a groupoid, then the multiplication map is a cover. Moreover, it splits.

Proof. The multiplication map is the following composition

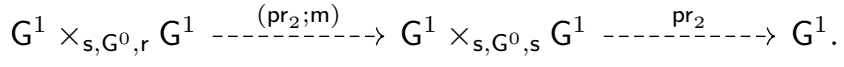

We know that the arrows $\left(\mathrm{pr}_{2} ; \mathrm{m}\right)$ as an isomorphism and $\mathrm{pr}_{2}$ as a pull-back of the source map are partial covers by Definition 2.1. Therefore, $\mathrm{m}$ is a partial cover. Also, we have that $\mathrm{m} \circ\left(\mathbf{u} \circ \mathrm{r} ; \mathrm{id}_{\mathrm{G}^{1}}\right)=\mathrm{id}_{\mathrm{G}^{1}}$. So $\mathrm{m}$ splits. Therefore, it is a cover by Lemma 2.5

Proposition 3.11. Let $\mathrm{G}^{0}$ and $\mathrm{G}^{1}$ be objects and let $\mathrm{r}: \mathrm{G}^{1} \rightarrow \mathrm{G}^{0}$, s: $\mathrm{G}^{1} \rightarrow \mathrm{G}^{0}$, $\mathrm{m}: \mathrm{G}^{1} \times_{\mathrm{s}, \mathrm{G}^{0}, \mathrm{r}} \mathrm{G}^{1} \rightarrow \mathrm{G}^{1}$, $\mathrm{u}: \mathrm{G}^{0} \rightarrow \mathrm{G}^{1}$ and $\mathrm{i}: \mathrm{G}^{1} \rightarrow \mathrm{G}^{1}$ be such that the properties (1), (2) and (3) in Definition 3.1 and all properties in Proposition 3.8 are satisfied. Then $\mathrm{G}=\left(\mathrm{G}^{\circ}, \mathrm{G}^{1}, \mathrm{r}, \mathrm{s}, \mathrm{m}\right)$ is a groupoid.

Proof. We just have to prove that the arrows

$$
\left(\mathrm{pr}_{2} ; \mathrm{m}\right): \mathrm{G}^{1} \times{ }_{\mathrm{s}, \mathrm{G}^{0}, \mathrm{r}} \mathrm{G}^{1} \longrightarrow \mathrm{G}^{1} \times{ }_{\mathrm{s}, \mathrm{G}^{0}, \mathrm{~s}} \mathrm{G}^{1}
$$

and

$$
\left(\mathrm{pr}_{1} ; \mathrm{m}\right): \mathrm{G}^{1} \times_{\mathrm{s}, \mathrm{G}^{0}, \mathrm{r}} \mathrm{G}^{1} \longrightarrow \mathrm{G}^{1} \times_{\mathrm{r}, \mathrm{G}^{0}, \mathrm{r}} \mathrm{G}^{1}
$$

are isomorphisms. We can directly name the inverse arrows of them. Let us show that these arrows are

$$
\left(\mathrm{m} \circ\left(\mathrm{pr}_{2} ; \mathrm{i} \circ \mathrm{pr}_{1}\right) ; \mathrm{pr}_{1}\right): \mathrm{G}^{1} \times_{\mathrm{s}, \mathrm{G}^{0}, \mathrm{~s}} \mathrm{G}^{1} \longrightarrow \mathrm{G}^{1} \times_{\mathrm{s}, \mathrm{G}^{0}, \mathrm{r}} \mathrm{G}^{1}, \quad\left(g ; g_{1}\right) \mapsto\left(g_{1} \cdot g^{-1} ; g\right),
$$

and

$$
\left(\mathrm{pr}_{1} ; \mathrm{m} \circ\left(\mathrm{i} \circ \mathrm{pr}_{1} ; \mathrm{pr}_{2}\right)\right): \mathrm{G}^{1} \times_{\mathrm{r}, \mathrm{G}^{0}, \mathrm{r}} \mathrm{G}^{1} \longrightarrow \mathrm{G}^{1} \times_{\mathrm{s}, \mathrm{G}^{0}, \mathrm{r}} \mathrm{G}^{1}, \quad\left(g ; g_{1}\right) \mapsto\left(g ; g^{-1} \cdot g_{1}\right),
$$

respectively. First of all, they are well-defined: in the first case, we have $\mathbf{s}\left(g_{1}\right)=$ $\mathbf{s}(g)=\mathbf{r}\left(g^{-1}\right)$, so $g_{1}$ and $g^{-1}$ are composable and $\mathbf{s}\left(g_{1} \cdot g^{-1}\right)=\mathbf{s}\left(g^{-1}\right)=\mathbf{r}(g)$; in 
the second case, we have $\mathbf{s}\left(g^{-1}\right)=\mathrm{r}(g)=\mathrm{r}\left(g_{1}\right)$, so $g^{-1}$ and $g_{1}$ are composable and $\mathbf{s}(g)=\mathrm{r}\left(g^{-1}\right)=\mathrm{r}\left(g^{-1} \cdot g_{1}\right)$. At this time we used the property (2) in Definition 3.1 and the property $(3)$ in Proposition 3.8

Now consider the composition:

$$
\begin{aligned}
\left(\mathrm{m} \circ\left(\mathrm{pr}_{2} ; \mathrm{i} \circ \mathrm{pr}_{1}\right) ; \mathrm{pr}_{1}\right)\left(\left(\mathrm{pr}_{2} ; \mathrm{m}\right)\left(g ; g_{1}\right)\right) & =\left(\mathrm{m} \circ\left(\mathrm{pr}_{2} ; \mathrm{i} \circ \mathrm{pr}_{1}\right) ; \mathrm{pr}_{1}\right)\left(g_{1} ; g \cdot g_{1}\right) \\
& =\left(\left(g \cdot g_{1}\right) \cdot g_{1}^{-1} ; g_{1}\right) \\
& =\left(g \cdot\left(g_{1} \cdot g_{1}^{-1}\right) ; g_{1}\right) \\
& =\left(g \cdot 1_{\mathrm{r}\left(g_{1}\right)} ; g_{1}\right) \\
& =\left(g \cdot 1_{\mathrm{s}(g)} ; g_{1}\right) \\
& =\left(g ; g_{1}\right)
\end{aligned}
$$

for all $g, g_{1} \in \mathrm{G}^{1}$ with $\mathrm{s}(g)=\mathrm{r}\left(g_{1}\right)$. Hence

$$
\left(\mathrm{m} \circ\left(\mathrm{pr}_{2} ; \mathrm{i} \circ \mathrm{pr}_{1}\right) ; \mathrm{pr}_{1}\right) \circ\left(\mathrm{pr}_{2} ; \mathrm{m}\right)=\mathrm{id}_{\left(\mathrm{G}^{1} \times_{\mathrm{s}, \mathrm{G}^{0}, \mathrm{r}} \mathrm{G}^{1}\right)} .
$$

Here we used the associativity of the multiplication map $m$ and the properties $(2)$ and (4) in Proposition 3.8 Also, we need the inverse composition:

$$
\begin{aligned}
\left(\mathrm{pr}_{2} ; \mathrm{m}\right)\left(\left(\mathrm{m} \circ\left(\mathrm{pr}_{2} ; \mathrm{i} \circ \mathrm{pr}_{1}\right) ; \mathrm{pr}_{1}\right)\left(g ; g_{1}\right)\right) & =\left(\mathrm{pr}_{2} ; \mathrm{m}\right)\left(g_{1} \cdot g^{-1} ; g\right) \\
& =\left(g ;\left(g_{1} \cdot g^{-1}\right) \cdot g\right) \\
& =\left(g ; g_{1} \cdot\left(g^{-1} \cdot g\right)\right) \\
& =\left(g ; g_{1} \cdot 1_{\mathrm{s}(g)}\right) \\
& =\left(g ; g_{1} \cdot 1_{\mathrm{s}\left(g_{1}\right)}\right) \\
& =\left(g ; g_{1}\right)
\end{aligned}
$$

for all $g, g_{1} \in \mathrm{G}^{1}$ with $\mathrm{s}(g)=\mathrm{s}\left(g_{1}\right)$. Hence $\left(\mathrm{pr}_{2} ; \mathrm{m}\right) \circ\left(\mathrm{m} \circ\left(\mathrm{pr}_{2} ; \mathrm{i} \circ \mathrm{pr}_{1}\right) ; \mathrm{pr}_{1}\right)=$

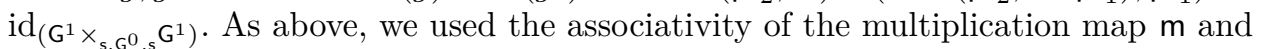
the properties (2) and (4) in Proposition 3.8 . So both compositions are identities and, therefore,

$$
\left(\mathrm{pr}_{2} ; \mathrm{m}\right): \mathrm{G}^{1} \times_{\mathrm{s}, \mathrm{G}^{0}, \mathrm{r}} \mathrm{G}^{1} \longrightarrow \mathrm{G}^{1} \times_{\mathrm{s}, \mathrm{G}^{0}, \mathrm{~s}} \mathrm{G}^{1}
$$

is an isomorphism.

THerefore, for the compositions $\left(\mathrm{pr}_{1} ; \mathrm{m} \circ\left(\mathrm{i} \circ \mathrm{pr}_{1} ; \mathrm{pr}_{2}\right)\right) \circ\left(\mathrm{pr}_{1} ; \mathrm{m}\right)$ and $\left(\mathrm{pr}_{1} ; \mathrm{m}\right) \circ$ $\left(\mathrm{pr}_{1} ; \mathrm{m} \circ\left(\mathrm{i} \circ \mathrm{pr}_{1} ; \mathrm{pr}_{2}\right)\right)$ is similar.

$$
\begin{aligned}
\left(\mathrm{pr}_{1} ; \mathrm{m} \circ\left(\mathrm{i} \circ \mathrm{pr}_{1} ; \mathrm{pr}_{2}\right)\right)\left(\left(\mathrm{pr}_{1} ; \mathrm{m}\right)\left(g ; g_{1}\right)\right) & =\left(\mathrm{pr}_{1} ; \mathrm{m} \circ\left(\mathrm{i} \circ \mathrm{pr}_{1} ; \mathrm{pr}_{2}\right)\right)\left(g ; g \cdot g_{1}\right) \\
& =\left(g ; g^{-1} \cdot\left(g \cdot g_{1}\right)\right) \\
& =\left(g ;\left(g^{-1} \cdot g\right) \cdot g_{1}\right) \\
& =\left(g ; 1_{\mathrm{s}(g)} \cdot g_{1}\right) \\
& =\left(g ; 1_{\mathrm{r}\left(g_{1}\right)} \cdot g_{1}\right) \\
& =\left(g ; g_{1}\right)
\end{aligned}
$$

for all $g, g_{1} \in \mathrm{G}^{1}$ with $\mathrm{s}(g)=\mathrm{r}\left(g_{1}\right)$. Hence

$$
\left(\mathrm{pr}_{1} ; \mathrm{m} \circ\left(\mathrm{i} \circ \mathrm{pr}_{1} ; \mathrm{pr}_{2}\right)\right) \circ\left(\mathrm{pr}_{1} ; \mathrm{m}\right)=\mathrm{id}_{\left(\mathrm{G}^{1} \times_{\mathrm{s}, \mathrm{G}^{0}, \mathrm{r}} \mathrm{G}^{1}\right)} .
$$

Also, for the second composition we have

$$
\begin{aligned}
\left(\mathrm{pr}_{1} ; \mathrm{m}\right)\left(\left(\mathrm{pr}_{1} ; \mathrm{m} \circ\left(\mathrm{i} \circ \mathrm{pr}_{1} ; \mathrm{pr}_{2}\right)\right)\left(g ; g_{1}\right)\right) & =\left(\mathrm{pr}_{1} ; \mathrm{m}\right)\left(g ; g^{-1} \cdot g_{1}\right) \\
& =\left(g ; g \cdot\left(g^{-1} \cdot g_{1}\right)\right) \\
& =\left(g ;\left(g \cdot g^{-1}\right) \cdot g_{1}\right) \\
& =\left(g ; 1_{\mathrm{r}(g)} \cdot g_{1}\right) \\
& =\left(g ; 1_{\mathrm{r}\left(g_{1}\right)} \cdot g_{1}\right) \\
& =\left(g ; g_{1}\right)
\end{aligned}
$$


for all $g, g_{1} \in \mathrm{G}^{1}$ with $\mathrm{r}(g)=\mathrm{r}\left(g_{1}\right)$. Hence

$$
\left(\mathrm{pr}_{1} ; \mathrm{m}\right) \circ\left(\mathrm{pr}_{1} ; \mathrm{m} \circ\left(\mathrm{i} \circ \mathrm{pr}_{1} ; \mathrm{pr}_{2}\right)\right)=\mathrm{id}_{\left(\mathrm{G}^{1} \times_{\mathrm{r}, \mathrm{G}^{0}, \mathrm{r}} \mathrm{G}^{1}\right)} .
$$

As for the arrow $\left(\mathrm{pr}_{2} ; \mathrm{m}\right)$, we used the properties (2) and (4) in Proposition 3.8 and the associativity of the multiplication map $\mathrm{m}$.

Remark 3.12. We did not use the property (1) in the proof, but it has to be required for the arrows in the property (2) to be well-defined.

Remark 3.13. If we require that the arrows $\mathrm{r}: \mathrm{G}^{1} \rightarrow \mathrm{G}^{0}$ and $\mathrm{s}: \mathrm{G}^{1} \rightarrow \mathrm{G}^{0}$ are partial covers instead of the property (1) in Definition 3.1 it would be enough because in this case the arrows $r: G^{1} \rightarrow G^{0}$ and $s: G^{1} \rightarrow G^{0}$ are partial covers which split by $\mathrm{u}: \mathrm{G}^{0} \rightarrow \mathrm{G}^{1}$ and therefore, they are covers by Lemma 2.5

\subsection{Examples of groupoids.}

Example 3.14. Let $\mathbf{X}$ be an object in $\mathcal{C}$. There is a groupoid with the object $\mathbf{X}$ as arrows and as objects, too. The source and range maps are $\mathrm{id}_{\mathrm{X}}: \mathrm{X} \rightarrow \mathrm{X}$, and the multiplication map is the obvious isomorphism $\operatorname{pr}_{1}: X \times_{\text {idx }_{X}, X, i_{X}} X \stackrel{\sim}{\rightarrow} X$. It is easy to check that this defines a groupoid. The unit and inverse maps are identity arrows like the source and range maps. We say that the object $X$ is viewed as a groupoid with only identity arrows.

Example 3.15. Let $f: \mathrm{X} \rightarrow \mathrm{Y}$ be a partial cover. Its Čech groupoid is the groupoid with $X$ as objects and $X \times_{f, Y, f} X$ as arrows. The range and source maps are $\mathrm{pr}_{1}: \mathrm{X} \times_{f, \mathrm{Y}, f} \mathrm{X} \rightarrow \mathrm{X}$ and $\mathrm{pr}_{2}: \mathrm{X} \times_{f, \mathrm{Y}, f} \mathrm{X} \rightarrow \mathrm{X}$, respectively. They are covers because of Corollary 2.8 The multiplication map is

$$
\left(\mathrm{X} \times_{f, \mathrm{Y}, f} \mathrm{X}\right) \times_{\mathrm{pr}_{2}, \mathrm{X}, \mathrm{pr}_{1}}\left(\mathrm{X} \times_{f, \mathrm{Y}, f} \mathrm{X}\right) \stackrel{\left(\mathrm{pr}_{1} \circ \mathrm{pr}_{1} ; \mathrm{pr}_{2} \circ \mathrm{pr}_{2}\right)}{\longrightarrow} \mathrm{X} \times_{f, \mathrm{Y}, f} \mathrm{X}
$$

defined elementwise by $\left(x_{1} ; x_{2}\right) \cdot\left(x_{2} ; x_{3}\right)=\left(x_{1} ; x_{3}\right)$ for all $x_{1}, x_{2}, x_{3} \in \mathbf{X}$ with $f\left(x_{1}\right)=f\left(x_{2}\right)=f\left(x_{3}\right)$. It is easy to check that this defines a groupoid. The unit map is

for all $x \in \mathbf{X}$ and the inverse map is

$$
\left(\operatorname{id}_{\mathbf{X}} ; \mathrm{id}_{\mathrm{X}}\right): \mathrm{X} \rightarrow \mathrm{X} \times_{f, \mathrm{Y}, f} \mathrm{X}, \quad x \mapsto(x ; x)
$$

$$
\left(\mathrm{pr}_{2} ; \mathrm{pr}_{1}\right): \mathrm{X} \times_{f, \mathrm{Y}, f} \mathrm{X} \rightarrow \mathrm{X} \times_{f, \mathrm{Y}, f} \mathrm{X}, \quad\left(x_{1} ; x_{2}\right) \mapsto\left(x_{2} ; x_{1}\right)
$$

for all $x_{1}, x_{2} \in \mathrm{X}$ with $f\left(x_{1}\right)=f\left(x_{2}\right)$.

Example 3.16. Let $\mathrm{G}$ be a groupoid and let $f: X \rightarrow G^{0}$ be a partial cover. We define a groupoid $\mathrm{G}(\mathrm{X}, f)$ with arrows $\left(\mathrm{X} \times_{f, \mathrm{G}^{0}, \mathrm{r}} \mathrm{G}^{1}\right) \times_{\mathrm{pr}_{2}, \mathrm{G}^{1}, \mathrm{pr}_{1}}\left(\mathrm{G}^{1} \times_{\mathrm{s}, \mathrm{G}^{0}, f} \mathrm{X}\right)$ and objects $\mathrm{X}$. The range and source maps are $\mathrm{pr}_{1} \circ \mathrm{pr}_{1}$ and $\mathrm{pr}_{2} \circ \mathrm{pr}_{2}$, respectively. The multiplication map is $\left(\left(\mathrm{pr}_{1} \circ \mathrm{pr}_{1} \circ \mathrm{pr}_{1} ; \hat{\mathrm{m}}\right) ;\left(\hat{\mathrm{m}} ; \mathrm{pr}_{2} \circ \mathrm{pr}_{2} \circ \mathrm{pr}_{2}\right)\right)$, where $\hat{\mathrm{m}}=\mathrm{mo}\left(\mathrm{pr}_{2} \circ \mathrm{pr}_{1} \circ \mathrm{pr}_{1} ; \mathrm{pr}_{2} \circ \mathrm{pr}_{1} \circ \mathrm{pr}_{2}\right)$. Elementwise, $\left(\left(x_{1} ; g\right) ;\left(g ; x_{2}\right)\right) \cdot\left(\left(x_{2} ; g_{1}\right) ;\left(g_{1} ; x_{3}\right)\right)=\left(\left(x_{1} ; g \cdot g_{1}\right) ;\left(g \cdot g_{1} ; x_{3}\right)\right)$. The inverse map is the arrow $\left(\left(\mathrm{pr}_{2} \circ \mathrm{pr}_{2} ; \mathrm{i} \circ \mathrm{pr}_{2} \circ \mathrm{pr}_{1}\right) ;\left(\mathrm{i} \circ \mathrm{pr}_{2} \circ \mathrm{pr}_{1} ; \mathrm{pr}_{1} \circ \mathrm{pr}_{1}\right)\right)$. Elementwise, $\left(\left(x_{1} ; g\right) ;\left(g ; x_{2}\right)\right) \mapsto\left(\left(x_{2} ; g^{-1}\right) ;\left(g^{-1} ; x_{1}\right)\right)$. The unit map is $\left(\left(i d x_{\mathbf{x}} ; \mathbf{u} \circ f\right) ;(\mathbf{u} \circ f ; \operatorname{idx})\right)$. Elementwise, $x \mapsto\left(\left(x ; 1_{f(x)}\right) ;\left(1_{f(x)} ; x\right)\right)$. Since

$$
\begin{aligned}
\mathrm{pr}_{1} \circ \mathrm{pr}_{1} \circ((\mathrm{idx} ; \mathrm{u} \circ f) ;(\mathrm{u} \circ f ; \mathrm{idx})) & =\mathrm{idx}_{\mathbf{x}} \\
& =\mathrm{pr}_{2} \circ \mathrm{pr}_{2} \circ\left(\left(\operatorname{idx}_{\mathbf{x}} ; \mathrm{u} \circ f\right) ;\left(\mathrm{u} \circ f ; \mathrm{idx}_{\mathbf{x}}\right)\right),
\end{aligned}
$$

the range and source maps are split partial covers, Hence they are covers by Lemma 2.5. It is easy to check that these arrows satisfy all conditions from Proposition 3.11 Hence $\mathrm{G}(\mathrm{X}, f)$ is a groupoid.

If the groupoid $\mathrm{G}$ in Example 3.16 is a groupoid with only identity arrows, then the groupoid $\mathrm{G}(\mathrm{X}, f)$ is the Čech groupoid of $f: \mathrm{X} \rightarrow \mathrm{G}^{0}$ defined in Example 3.15 .

If $f_{1}: \mathbf{X}_{1} \rightarrow \mathrm{X}$ is a partial cover then there is a natural groupoid isomorphism

$$
\mathrm{G}\left(\mathrm{X}_{1}, f \circ f_{1}\right) \cong \mathrm{G}(\mathrm{X}, f)\left(\mathrm{X}_{1}, f_{1}\right) \text {. }
$$


Example 3.17. Assume Assumption 2.20 A group is a groupoid $\mathrm{G}=\left(\mathrm{G}^{0}, \mathrm{G}^{1}, \mathrm{r}, \mathrm{s}, \mathrm{m}\right)$, where $\mathrm{G}^{0}$ is the final object.

\section{Morphisms BetWeEn GRoupoids}

The usual morphisms between groupoids in a category $\left(\mathcal{C}, \mathcal{F}_{p}\right)$ with partial covers are functors. They form a category with the groupoids as objects.

Definition 4.1. Let $\mathrm{L}$ and $\mathrm{H}$ be groupoids in $\left(\mathcal{C}, \mathcal{F}_{p}\right)$. A functor from $\mathrm{L}$ to $\mathrm{H}$ is given by arrows $\mathrm{F}^{0}: \mathrm{L}^{0} \rightarrow \mathrm{H}^{0}$ and $\mathrm{F}^{1}: \mathrm{L}^{1} \rightarrow \mathrm{H}^{1}$ that intertwine the source, range and multiplication maps. That is,

(1) $\mathrm{F}^{0} \circ \mathrm{s}_{\mathrm{L}}=\mathrm{s}_{\mathrm{H}} \circ \mathrm{F}^{1} ; \quad \mathrm{F}^{0}\left(\mathrm{~s}_{\mathrm{L}}(l)\right)=\mathrm{s}_{\mathrm{H}}\left(\mathrm{F}^{1}(l)\right), \quad \forall l \in \mathrm{L}^{1} ;$

(2) $\mathrm{F}^{0} \circ \mathrm{r}_{\mathrm{L}}=\mathrm{r}_{\mathrm{H}} \circ \mathrm{F}^{1} ; \quad \mathrm{F}^{0}\left(\mathrm{r}_{\mathrm{L}}(l)\right)=\mathrm{r}_{\mathrm{H}}\left(\mathrm{F}^{1}(l)\right), \quad \forall l \in \mathrm{L}^{1}$;

(3) $\mathrm{F}^{1} \circ \mathrm{m}_{\mathrm{L}}=\mathrm{m}_{\mathrm{H}} \circ\left(\mathrm{F}^{1} \circ \mathrm{pr}_{1} ; \mathrm{F}^{1} \circ \mathrm{pr}_{2}\right) ; \quad \mathrm{F}^{1}\left(l \cdot l_{1}\right)=\mathrm{F}^{1}(l) \cdot \mathrm{F}^{1}\left(l_{1}\right)$ for all $l, l_{1} \in \mathrm{L}^{1}$ with $\mathrm{s}_{\mathrm{L}}(l)=\mathrm{r}_{\mathrm{L}}\left(l_{1}\right)$; here $\mathrm{pr}_{1}$ and $\mathrm{pr}_{2}$ are the coordinate projections $\mathrm{pr}_{1}, \mathrm{pr}_{2}: \mathrm{L}^{1} \times_{\mathrm{S}_{\mathrm{L}}, \mathrm{L}^{0}, \mathrm{r}_{\mathrm{L}}} \mathrm{L}^{1} \rightrightarrows \mathrm{L}^{1}$.

The composition of functors $\mathrm{F}: \mathrm{L} \rightarrow \mathrm{H}$ and $\mathrm{E}: \mathrm{H} \rightarrow \mathrm{R}$ is the functor $\mathrm{E} \circ \mathrm{F}: \mathrm{L} \rightarrow \mathrm{R}$ which is given by the composite arrows $(E \circ F)^{0}=E^{0} \circ F^{0}$ and $(E \circ F)^{1}=E^{1} \circ F^{1}$. We just need to check that this composition intertwines the source, range and multiplication maps of the groupoids $L$ and $R$. It is so because

$$
\begin{aligned}
(\mathrm{E} \circ \mathrm{F})^{0} \circ \mathrm{S}_{\mathrm{L}} & =\mathrm{E}^{0} \circ \mathrm{F}^{0} \circ \mathrm{S}_{\mathrm{L}} \\
& =\mathrm{E}^{0} \circ \mathrm{S}_{\mathrm{H}} \circ \mathrm{F}^{1} \\
& =\mathrm{S}_{\mathrm{R}} \circ \mathrm{E}^{1} \circ \mathrm{F}^{1} \\
& =\mathrm{S}_{\mathrm{R}} \circ(\mathrm{E} \circ \mathrm{F})^{1} .
\end{aligned}
$$

Analogously,

$$
\begin{aligned}
(E \circ F)^{0} \circ r_{L} & =E^{0} \circ F^{0} \circ r_{L} \\
& =E^{0} \circ r_{H} \circ F^{1} \\
& =r_{R} \circ E^{1} \circ F^{1} \\
& =r_{R} \circ(E \circ F)^{1} .
\end{aligned}
$$

Also, for the multiplication maps we have

$$
\begin{aligned}
(\mathrm{E} \circ \mathrm{F})^{1} \circ \mathrm{m}_{\mathrm{L}} & =\mathrm{E}^{1} \circ \mathrm{F}^{1} \circ \mathrm{m}_{\mathrm{L}} \\
& =\mathrm{E}^{1} \circ \mathrm{m}_{\mathrm{H}} \circ\left(\mathrm{F}^{1} \circ \mathrm{pr}_{1} ; \mathrm{F}^{1} \circ \mathrm{pr}_{2}\right) \\
& =\mathrm{m}_{\mathrm{R}} \circ\left(\mathrm{E}^{1} \circ \mathrm{pr}_{1} ; \mathrm{E}^{1} \circ \mathrm{pr}_{2}\right) \circ\left(\mathrm{F}^{1} \circ \mathrm{pr}_{1} ; \mathrm{F}^{1} \circ \mathrm{pr}_{2}\right) \\
& =\mathrm{m}_{\mathrm{R}} \circ\left(\mathrm{E}^{1} \circ \mathrm{F}^{1} \circ \mathrm{pr}_{1} ; \mathrm{E}^{1} \circ \mathrm{F}^{1} \circ \mathrm{pr}_{2}\right) \\
& =\mathrm{m}_{\mathrm{R}} \circ\left((\mathrm{E} \circ \mathrm{F})^{1} \circ \mathrm{pr}_{1} ;(\mathrm{E} \circ \mathrm{F})^{1} \circ \mathrm{pr}_{2}\right) .
\end{aligned}
$$

It is clear that the functor $\mathrm{id}_{\mathrm{L}}: \mathrm{L} \rightarrow \mathrm{L}$ given by the arrows $\operatorname{id}_{\mathrm{L}^{0}}$ and $\operatorname{id}_{\mathrm{L}^{1}}$ is an identity functor on the groupoid L. So groupoids and functors between them form a category.

Lemma 4.2. Any functor $\mathrm{F}: \mathrm{L} \rightarrow \mathrm{H}$ intertwines the unit and inverse maps of the groupoids $\mathrm{L}$ and $\mathrm{H}$.

Proof. For any element $l \in \mathrm{L}^{1}$ the pair $\left(\mathrm{F}^{1}(l) ; \mathrm{F}^{1}\left(1_{\mathrm{s}(l)}\right)\right)$ is composable in $\mathrm{H}$ because $\mathrm{s}_{\mathrm{H}}\left(\mathrm{F}^{1}(l)\right)=\mathrm{F}^{0}\left(\mathrm{~s}_{\mathrm{L}}(l)\right)=\mathrm{F}^{0}\left(\mathrm{r}_{\mathrm{L}}\left(1_{\mathrm{s}_{\mathrm{L}}(l)}\right)\right)=\mathrm{r}_{\mathrm{H}}\left(\mathrm{F}^{1}\left(1_{\mathrm{s}_{\mathrm{L}}(l)}\right)\right)$. We have

$$
\begin{aligned}
\mathrm{F}^{1}(l) \cdot \mathrm{F}^{1}\left(1_{\mathrm{s}(l)}\right) & =\mathrm{F}^{1}\left(l \cdot 1_{\mathrm{s}(l)}\right) \\
& =\mathrm{F}^{1}(l) \\
& =\mathrm{F}^{1}(l) \cdot 1_{\mathrm{SH}_{(}\left(\mathrm{F}^{1}(l)\right)} \\
& =\mathrm{F}^{1}(l) \cdot 1_{\mathrm{F}^{0}\left(\mathrm{~s}_{\mathrm{L}}(l)\right)} .
\end{aligned}
$$


Hence $\mathrm{F}^{1}\left(1_{\mathrm{s}(l)}\right)=1_{\mathrm{F}^{0}\left(\mathrm{~s}_{\mathrm{L}}(l)\right)}$ by Remark 3.6. Since the source map is epic, we have $\mathrm{F}^{1}\left(1_{l_{0}}\right)=1_{\mathrm{F}^{0}\left(l_{0}\right)}$, for all $l_{0} \in \mathrm{L}^{0}$. So $\mathrm{F}: \mathrm{L} \rightarrow \mathrm{H}$ intertwines the unit maps.

Also, for any element $l \in \mathrm{L}^{1}$ the pair $\left(\mathrm{F}^{1}(l) ; \mathrm{F}^{1}\left(l^{-1}\right)\right)$ is composable in $\mathrm{H}$ because $\mathrm{s}_{\mathrm{H}}\left(\mathrm{F}^{1}(l)\right)=\mathrm{F}^{1}\left(\mathrm{~s}_{\mathrm{L}}(l)\right)=\mathrm{F}^{1}\left(\mathrm{r}_{\mathrm{L}}\left(l^{-1}\right)\right)=\mathrm{r}_{\mathrm{H}}\left(\mathrm{F}^{1}\left(l^{-1}\right)\right)$. We have

$$
\begin{aligned}
& \mathrm{F}^{1}(l) \cdot \mathrm{F}^{1}\left(l^{-1}\right)=\mathrm{F}^{1}\left(l \cdot l^{-1}\right) \\
& =\mathrm{F}^{1}\left(1_{\mathrm{r}_{\mathrm{L}}(l)}\right) \\
& =1_{\mathrm{F}^{0}\left(\mathrm{r}_{\mathrm{L}}(l)\right)}=1_{\mathrm{r}_{\mathrm{H}}\left(\mathrm{F}^{1}(l)\right)} \\
& =\mathrm{F}^{1}(l) \cdot\left(\mathrm{F}^{1}(l)\right)^{-1} \text {. }
\end{aligned}
$$

Hence $\mathrm{F}^{1}\left(l^{-1}\right)=\left(\mathrm{F}^{1}(l)\right)^{-1}$, for all $l \in \mathrm{L}^{1}$ by Remark 3.6. So $\mathrm{F}: \mathrm{L} \rightarrow \mathrm{H}$ intertwines the inverse maps.

Definition 4.3. The groupoids $L$ and $H$ are called isomorphic if there is a functor $\mathrm{F}: \mathrm{L} \rightarrow \mathrm{H}$ such that $\mathrm{F}^{1}$ and $\mathrm{F}^{0}$ are isomorphisms. Such functors are called isomorphisms between groupoids.

It is easy to check that if $\mathrm{F}$ is an isomorphism from $\mathrm{L}$ to $\mathrm{H}$ then the pair $\left(\left(\mathrm{F}^{1}\right)^{-1} ;\left(\mathrm{F}^{0}\right)^{-1}\right)=\mathrm{F}^{-1}$ defines a functor from $\mathrm{H}$ to $\mathrm{L}$. Hence it is an isomorphism too.

Lemma 4.4. The groupoid $\mathrm{L}$ is isomorphic to the groupoid with only identity arrows (see Example 3.14) if and only if its source map is an isomorphism. Such groupoids are called 0-groupoid.

Proof. Let $\mathrm{F}$ be a isomorphism from $\mathrm{L}=\left(\mathrm{L}^{0}, \mathrm{~L}^{1}, \mathrm{r}, \mathrm{s}, \mathrm{m}\right)$ to $\mathrm{X}=\left(\mathrm{X}, \mathrm{X}, \mathrm{id} \mathrm{X}, \mathrm{id} \mathrm{X}, \mathrm{pr}_{1}\right)$. Then the arrow $\left(\mathrm{F}^{1}\right)^{-1} \circ \mathrm{F}^{0}: \mathrm{L}^{0} \rightarrow \mathrm{L}^{1}$ is an inverse of the source map, because

$$
\begin{aligned}
\left(\mathrm{F}^{1}\right)^{-1} \circ \mathrm{F}^{0} \circ \mathrm{s} & =\left(\mathrm{F}^{1}\right)^{-1} \circ \mathrm{id} \times \mathrm{F}^{1} \\
& =\left(\mathrm{F}^{1}\right)^{-1} \circ \mathrm{F}^{1} \\
& =\mathrm{id}_{\mathrm{L}^{1}}
\end{aligned}
$$

and

$$
\begin{aligned}
\mathrm{s} \circ\left(\mathrm{F}^{1}\right)^{-1} \circ \mathrm{F}^{0} & =\left(\mathrm{F}^{0}\right)^{-1} \circ \mathrm{id} \mathrm{X} \circ \mathrm{F}^{0} \\
& =\left(\mathrm{F}^{0}\right)^{-1} \circ \mathrm{F}^{0} \\
& =\mathrm{id}_{\mathrm{L}^{0}} .
\end{aligned}
$$

So the source map is an isomorphism and equal to the range map because

$$
\begin{aligned}
\mathrm{s} & =\mathrm{s} \circ \mathrm{F}^{0} \circ\left(\mathrm{F}^{0}\right)^{-1} \\
& =\mathrm{F}^{1} \circ \mathrm{id} \times\left(\mathrm{F}^{0}\right)^{-1} \\
& =r \circ \mathrm{F}^{0} \circ\left(\mathrm{F}^{0}\right)^{-1} \\
& =r .
\end{aligned}
$$

Conversely, let the source map be an isomorphism. Then $\mathrm{s}^{-1}=\mathrm{u}$ because

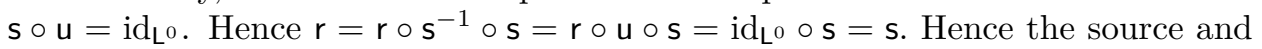
range maps are the same. Now it is easy to check that the pair of isomorphisms $\left(\mathrm{s} ; \mathrm{id}_{\mathrm{L}^{0}}\right)$ intertwines the source and range maps of the groupoids $L=\left(\mathrm{L}^{0}, \mathrm{~L}^{1}, \mathrm{r}, \mathrm{s}, \mathrm{m}\right)$ and $\mathrm{L}^{0}=\left(\mathrm{L}^{0}, \mathrm{~L}^{0}, \mathrm{id}_{\mathrm{L}^{0}}, \mathrm{id}_{\mathrm{L}^{0}}, \mathrm{pr}_{1}\right)$. It intertwines the multiplication maps too because

$$
\begin{aligned}
\mathrm{s}\left(g \cdot g_{1}\right) & =\mathrm{s}\left(g_{1}\right) \\
& =\mathrm{r}\left(g_{1}\right) \\
& =\mathrm{r}\left(g_{1}\right) \cdot \mathrm{r}\left(g_{1}\right) \\
& =\mathrm{s}(g) \cdot \mathrm{s}\left(g_{1}\right)
\end{aligned}
$$

for all $g, g_{1} \in \mathrm{G}^{1}$ with $\mathrm{s}(g)=\mathrm{r}\left(g_{1}\right)$. Therefore, $\left(\mathrm{s} ; \mathrm{id}_{\mathrm{L}^{0}}\right)$ is an isomorphism from $\mathrm{L}$ to $\mathrm{L}^{0}$. 
Remark 4.5. The proof shows that for any 0-goupoid the source and range maps are isomorphisms and the unit map is an inverse of them.

4.1. Groupoid fibrations. We consider special functors which form a full subcategory of the category of groupoids. For topological groupoids, groupoid fibrations are studied in 10 .

Definition 4.6. Let $\mathrm{L}$ and $\mathrm{H}$ be groupoids and let $\mathrm{F}: \mathrm{L} \rightarrow \mathrm{H}$ be a functor between them. We call F a partial groupoid fibration, groupoid fibration, partial groupoid covering or groupoid covering if the arrow

$$
\mathrm{L}^{1} \stackrel{\left(\mathrm{F}^{1} ; \mathrm{s}_{\mathrm{L}}\right)}{\longrightarrow} \mathrm{H}^{1} \times_{\mathrm{SH}_{\mathrm{H}}, \mathrm{H}^{0}, \mathrm{~F}^{0}} \mathrm{~L}^{0}
$$

is a partial cover, cover, monic partial cover or isomorphism, respectively.

The arrow (4.1) is well-defined because a functor between groupoids intertwines the source maps of the groupoids $\mathrm{L}$ and $\mathrm{H}$, that is $\mathrm{S}_{\mathrm{H}} \circ \mathrm{F}^{1}=\mathrm{F}^{0} \circ \mathrm{s}_{\mathrm{L}}$.

Remark 4.7. Any groupoid covering is a groupoid fibration, any groupoid fibration is a partial groupoid fibration and any groupoid covering is a partial groupoid covering.

Remark 4.8. If a functor between groupoids is a groupoid fibration and a partial groupoid covering, then it is a groupoid covering. That follows easily from Lemma 2.11

Lemma 4.9. Let $\mathrm{F}: \mathrm{L} \rightarrow \mathrm{H}$ be a functor between groupoids. Then the arrow

$$
\mathrm{L}^{1} \stackrel{\left(\mathrm{F}^{1} ; \mathrm{sL}_{\mathrm{L}}\right)}{\longrightarrow} \mathrm{H}^{1} \times_{\mathrm{sH}_{\mathrm{H}}, \mathrm{H}^{0}, \mathrm{~F}^{0}} \mathrm{~L}^{0}
$$

is a partial cover, cover, isomorphism or monic if and only if the arrow

$$
\mathrm{L}^{1} \stackrel{\left(\mathrm{F}^{1} ; \mathrm{r}_{\mathrm{L}}\right)}{\longrightarrow} \mathrm{H}^{1} \times_{\mathrm{rH}_{\mathrm{H}}, \mathrm{H}^{0}, \mathrm{~F}^{\circ}} \mathrm{L}^{0}
$$

is a partial cover, cover, isomorphism or monic, respectively.

Proof. Since $\mathrm{r}_{\mathrm{H}} \circ \mathrm{i}_{\mathrm{H}}=\mathrm{s}_{\mathrm{H}}$, there is a rectangle of pull-back squares

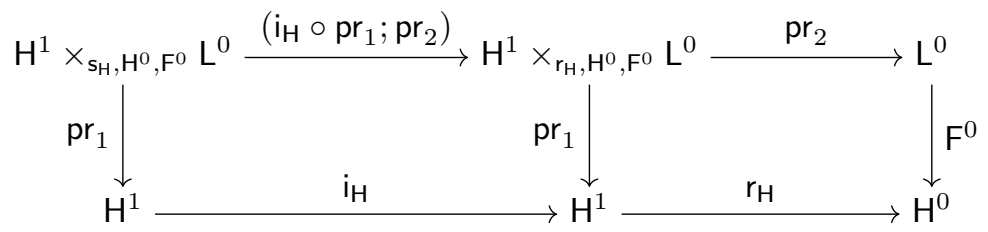

By using the well-known lemma about the rectangle of pull-back squares we can say that the left-side square is a pull-back square. Therefore, the arrow

$$
\mathrm{H}^{1} \times_{\mathrm{SH}_{\mathrm{H}}, \mathrm{H}^{0}, \mathrm{~F}^{0}} \mathrm{~L}^{0} \stackrel{\left(\mathrm{i}_{\mathrm{H}} \mathrm{O} \mathrm{pr}_{1} ; \mathrm{pr}_{2}\right)}{\longrightarrow} \mathrm{H}^{1} \times_{\mathrm{r}_{\mathrm{H}}, \mathrm{H}^{0}, \mathrm{~F}^{0}} \mathrm{~L}^{0}
$$

is an isomorphism because it is the pull-back of the inverse map of the groupoid $\mathrm{H}$, which is an isomorphism because of (2) in Corollary 3.9

Also, we have

$$
\begin{aligned}
\left(\mathrm{i}_{\mathrm{H}} \circ \mathrm{pr}_{1} ; \mathrm{pr}_{2}\right) \circ\left(\mathrm{F}^{1} ; \mathrm{s}_{\mathrm{L}}\right) & =\left(\mathrm{i}_{\mathrm{H}} \circ \mathrm{F}^{1} ; \mathrm{s}_{\mathrm{L}}\right) \\
& =\left(\mathrm{F}^{1} \circ \mathrm{i}_{\mathrm{L}} ; \mathrm{r}_{\mathrm{L}} \circ \mathrm{i}_{\mathrm{L}}\right) \\
& =\left(\mathrm{F}^{1} ; \mathrm{r}_{\mathrm{L}}\right) \circ \mathrm{i}_{\mathrm{L}} .
\end{aligned}
$$


So we have the commuting diagram

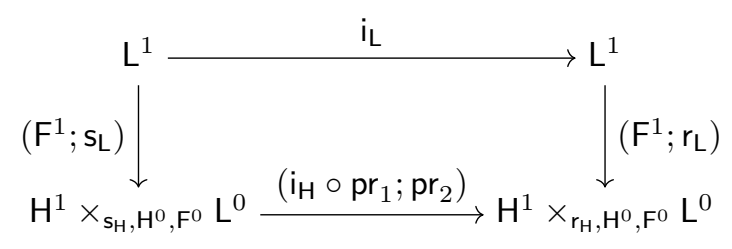

such that the horizontal arrows are isomorphisms. This finishes the proof of the lemma.

Proposition 4.10. A composite of partial groupoid fibrations is a partial groupoid fibration.

Proof. Let $\mathrm{F}: \mathrm{L} \rightarrow \mathrm{H}$ and $\mathrm{E}: \mathrm{H} \rightarrow \mathrm{R}$ be partial groupoid fibrations. We have two partial covers

$$
L^{1}-\left(F^{1} ; S_{L}\right)-\longrightarrow \rightarrow H^{1} \times_{S_{H}, H^{0}, F^{0}} L^{0}
$$

and

$$
H^{1}-\left(E^{1} ; S_{H}\right)-\rightarrow R^{1} \times_{S_{R}, R^{0}, E^{0}} H^{0}
$$

by Definition 4.6 . We need to prove that the arrow

$$
\mathrm{L}^{1} \stackrel{\left(\mathrm{E}^{1} \circ \mathrm{F}^{1} ; \mathrm{S}_{\mathrm{L}}\right)}{\longrightarrow} \mathrm{R}^{1} \times_{\mathrm{SR}_{\mathrm{R}}, \mathrm{R}^{0}, \mathrm{E}^{0} \circ \mathrm{F}^{0}} \mathrm{~L}^{0}
$$

is a partial cover, too, which implies that the composition $\mathrm{E} \circ \mathrm{F}: \mathrm{L} \rightarrow \mathrm{H} \rightarrow \mathrm{R}$ is a partial groupoid fibration.

By using the well-known lemma about the rectangle of pull-back squares we can construct the following diagram, where each square is a pull-back square.

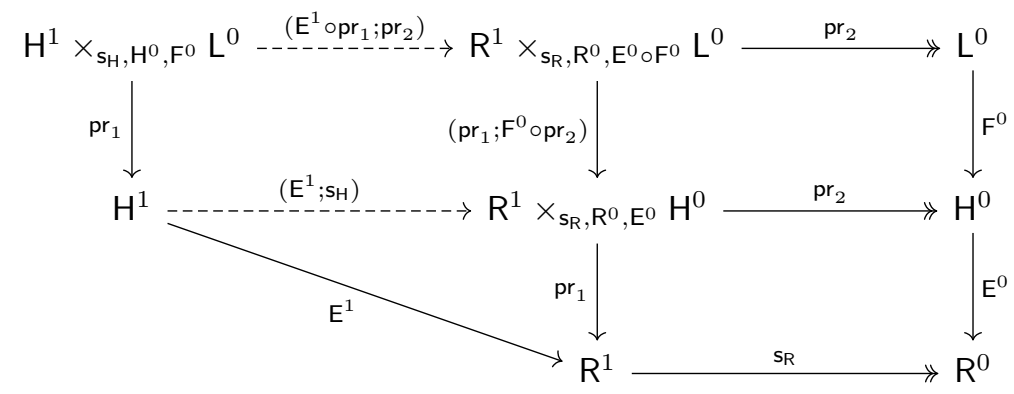

The arrow

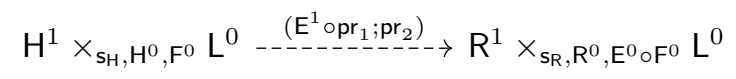

is a partial cover, because it is a pull-back of the arrow $\left(\mathrm{E}^{1} ; \mathrm{s}_{\mathrm{H}}\right)$. Therefore, the composition

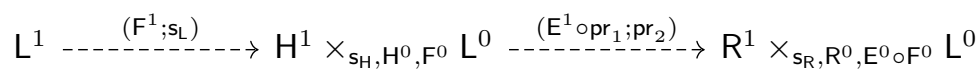

is a partial cover. We have

$$
\begin{aligned}
\left(\mathrm{E}^{1} \circ \mathrm{pr}_{1} ; \mathrm{pr}_{2}\right) \circ\left(\mathrm{F}^{1} ; \mathrm{s}_{\mathrm{L}}\right) & =\left(\mathrm{E}^{1} \circ\left(\mathrm{pr}_{1} \circ\left(\mathrm{F}^{1} ; \mathrm{s}_{\mathrm{L}}\right)\right) ; \mathrm{pr}_{2} \circ\left(\mathrm{F}^{1} ; \mathrm{s}_{\mathrm{L}}\right)\right) \\
& =\left(\mathrm{E}^{1} \circ \mathrm{F}^{1} ; \mathrm{s}_{\mathrm{L}}\right) .
\end{aligned}
$$

Hence the composition $\mathrm{E} \circ \mathrm{F}: \mathrm{L} \rightarrow \mathrm{H} \rightarrow \mathrm{R}$ is a partial groupoid fibration. 
Remark 4.11. The composition of two groupoid coverings is a groupoid covering. The proof is absolutely same. We just use that a pull-back of an isomorphism is an isomorphism and a composition of isomorphisms is an isomorphism. Similarly, a composition of partial groupoid coverings is a partial groupoid covering.

Remark 4.12. Under Assumptions 2.14 and 2.15 compositions of groupoid fibrations are groupoid fibrations, too. The proof is the same as the proof of Proposition 4.10 if we use the term "cover" instead of the term "partial cover".

The identity functor $\left(\operatorname{id}_{\mathrm{L}^{1}} ; \operatorname{id}_{\mathrm{L}^{0}}\right): \mathrm{L} \rightarrow \mathrm{L}$ is a groupoid covering because, in this case,

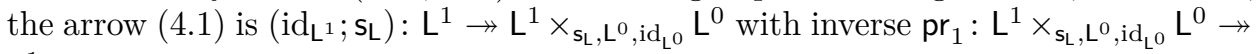
$\mathrm{L}^{1}$. Therefore, it is an identity morphism for each kind of functors defined in Definition 4.6. So, under relevant assumptions, we have four full subcategories of the category of groupoids with functors as arrows between them.

The next goal is to define the fibre of a partial groupoid fibration.

Definition 4.13. Let $\mathrm{F}: \mathrm{L} \rightarrow \mathrm{H}$ be a partial groupoid fibration. A fibre $\mathrm{G}$ consists of

- objects

(1) $\mathrm{G}^{1}=\mathrm{L}^{1} \times\left(\mathrm{F}^{1} ; \mathrm{s}_{\mathrm{L}}\right),\left(\mathrm{H}^{1} \times_{\mathrm{s}_{\mathrm{H}}, \mathrm{H}^{0}, \mathrm{~F}^{0}} \mathrm{~L}^{0}\right),\left(\mathrm{F}^{1} \mathrm{ou}_{\mathrm{L}} ; \mathrm{id}_{\mathrm{L} 0}\right) \mathrm{L}^{0}, \quad$ elements of this object are the pairs $\left(l, l_{0}\right), l \in \mathrm{L}^{1}, l_{0} \in \mathrm{L}^{0}$ with $\mathrm{s}_{\mathrm{L}}(l)=l_{0}$ and $\mathrm{F}^{1}(l)=\mathrm{F}^{1}\left(1_{\mathrm{s}_{\mathrm{L}}(l)}\right)$;

(2) $\mathrm{G}^{0}=\mathrm{L}^{0}$,

- arrows

(1) $\mathrm{s}_{\mathrm{G}}=\mathrm{s}_{\mathrm{L}} \circ \mathrm{pr}_{1}: \mathrm{G}^{1} \rightarrow \mathrm{G}^{0}, \quad \mathrm{~s}_{\mathrm{G}}\left(l ; l_{0}\right)=\mathrm{s}_{\mathrm{L}}(l), \quad \forall\left(l ; l_{0}\right) \in \mathrm{G}^{1}$

(2) $\mathrm{r}_{\mathrm{G}}=\mathrm{r}_{\mathrm{L}} \circ \mathrm{pr}_{1}: \mathrm{G}^{1} \rightarrow \mathrm{G}^{0}, \quad \mathrm{r}_{\mathrm{G}}\left(l ; l_{0}\right)=\mathrm{r}_{\mathrm{L}}(l), \quad \forall\left(l ; l_{0}\right) \in \mathrm{G}^{1}$;

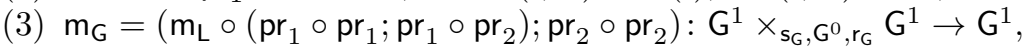
$\left(l ; l_{0}\right) \cdot\left(l^{\prime} ; l_{0}^{\prime}\right)=\left(l \cdot l^{\prime} ; l_{0}^{\prime}\right) \quad \forall\left(l ; l_{0}\right),\left(l^{\prime} ; l_{0}^{\prime}\right) \in \mathrm{G}^{1}$ with $\mathrm{s}_{\mathrm{G}}\left(l ; l_{0}\right)=\mathrm{r}_{\mathrm{G}}\left(l^{\prime} ; l_{0}^{\prime}\right)$.

Lemma 4.14. If we have the data above then there are two important equalities:

(1) $\mathrm{s}_{\mathrm{L}} \circ \mathrm{pr}_{1}=\mathrm{pr}_{2}, \quad \mathrm{~s}_{\mathrm{L}}(l)=l_{0}, \quad \forall\left(l ; l_{0}\right) \in \mathrm{G}^{1}$;

(2) $\mathrm{F}^{1} \circ \mathrm{pr}_{1}=\mathrm{F}^{1} \circ \mathrm{u}_{\mathrm{L}} \circ \mathrm{s}_{\mathrm{L}} \circ \mathrm{pr}_{1}, \quad \mathrm{~F}^{1}(l)=\mathrm{F}^{1}\left(1_{\mathrm{s}_{\mathrm{L}}(l)}\right), \quad \forall\left(l ; l_{0}\right) \in \mathrm{G}^{1}$;

Proof. We have

$$
\begin{aligned}
\left(\mathrm{F}^{1} \circ \mathrm{pr}_{1} ; \mathrm{s}_{\mathrm{L}} \circ \mathrm{pr}_{1}\right) & =\left(\mathrm{F}^{1} ; \mathrm{s}_{\mathrm{L}}\right) \circ \mathrm{pr}_{1} \\
& =\left(\mathrm{F}^{1} \circ \mathrm{u}_{\mathrm{L}} ; \mathrm{id}_{\mathrm{L}^{0}}\right) \circ \mathrm{pr}_{2} \\
& =\left(\mathrm{F}^{1} \circ \mathrm{u}_{\mathrm{L}} \circ \mathrm{pr}_{2} ; \mathrm{pr}_{2}\right) .
\end{aligned}
$$

Therefore, $s_{\mathrm{L}} \circ \mathrm{pr}_{1}=\mathrm{pr}_{2}$ and $\mathrm{F}^{1} \circ \mathrm{pr}_{1}=\mathrm{F}^{1} \circ \mathrm{u}_{\mathrm{L}} \circ \mathrm{pr}_{2}$. Hence $\mathrm{F}^{1} \circ \mathrm{pr}_{1}=\mathrm{F}^{1} \circ \mathrm{u}_{\mathrm{L}} \circ \mathrm{s}_{\mathrm{L}} \circ \mathrm{pr}_{1}$.

Proposition 4.15. The data $\mathrm{G}=\left(\mathrm{G}^{0}, \mathrm{G}^{1}, \mathrm{r}_{\mathrm{G}}, \mathrm{s}_{\mathrm{G}}, \mathrm{m}_{\mathrm{G}}\right)$ in Definition 4.13 is a welldefined groupoid.

Proof. Firstly we have to check that the arrow

$$
\mathrm{L}^{0} \stackrel{\left(\mathrm{F}^{1} \mathrm{ou}_{\mathrm{L}} ; \mathrm{id}_{\mathrm{L}^{0}}\right)}{\longrightarrow} \mathrm{H}^{1} \times_{\mathrm{SH}_{\mathrm{H}}, \mathrm{H}^{0}, \mathrm{~F}^{\mathrm{O}} \mathrm{L}^{0}}
$$

is well-defined. It is so because

$$
\begin{aligned}
\mathrm{s}_{\mathrm{H}} \circ\left(\mathrm{F}^{1} \circ \mathrm{u}_{\mathrm{L}}\right) & =\left(\mathrm{s}_{\mathrm{H}} \circ \mathrm{F}^{1}\right) \circ \mathrm{u}_{\mathrm{L}} \\
& =\left(\mathrm{F}^{0} \circ \mathrm{s}_{\mathrm{L}}\right) \circ \mathrm{u}_{\mathrm{L}} \\
& =\mathrm{F}^{0} \circ\left(\mathrm{s}_{\mathrm{L}} \circ \mathrm{u}_{\mathrm{L}}\right) \\
& =\mathrm{F}^{0} \circ \mathrm{id}_{\mathrm{L}^{0}} .
\end{aligned}
$$

The arrow

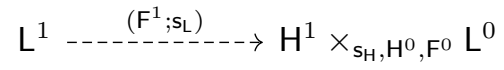


is a partial cover by Definition 4.6. Therefore, the fiber product

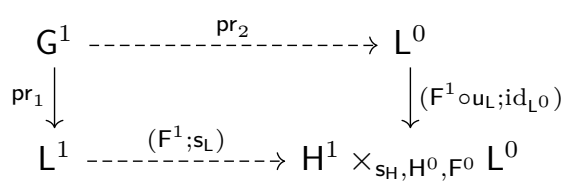

exists and the coordinate projection $\mathrm{pr}_{2}: \mathrm{G}^{1} \rightarrow \mathrm{L}^{0}$ is a partial cover too. We also have

$$
\begin{aligned}
\left(\mathrm{F}^{1} ; \mathrm{s}_{\mathrm{L}}\right) \circ \mathrm{u}_{\mathrm{L}} & =\left(\mathrm{F}^{1} \circ \mathrm{u}_{\mathrm{L}} ; \mathrm{s}_{\mathrm{L}} \circ \mathrm{u}_{\mathrm{L}}\right) \\
& =\left(\mathrm{F}^{1} \circ \mathrm{u}_{\mathrm{L}} ; \mathrm{id}_{\mathrm{L}^{0}}\right) \\
& =\left(\mathrm{F}^{1} \circ \mathrm{u}_{\mathrm{L}} ; \mathrm{id}_{\mathrm{L}^{0}}\right) \circ \mathrm{id}_{\mathrm{L}^{0}} .
\end{aligned}
$$

This means that there is a well-defined arrow $\left(\mathrm{u}_{\mathrm{L}} ; \mathrm{id}_{\mathrm{L}^{0}}\right): \mathrm{L}^{0} \rightarrow \mathrm{G}^{1}$. Since $\mathrm{pr}_{2} \circ$ $\left(\mathrm{u}_{\mathrm{L}} ; \mathrm{id}_{\mathrm{L}^{0}}\right)=\mathrm{id}_{\mathrm{L}^{0}}$, the coordinate projection $\mathrm{pr}_{2}: \mathrm{G}^{1} \rightarrow-\mathrm{L}^{0}$ is a partial cover which splits by $\left(\mathrm{u}_{\mathrm{L}} ; \mathrm{id}_{\mathrm{L}^{0}}\right)$. Hence it is a cover by Lemma 2.5 . Now we can infer that the source map $\mathrm{S}_{\mathrm{G}}: \mathrm{G}^{1} \rightarrow \mathrm{G}^{0}$ is a cover too because $\mathrm{S}_{\mathrm{G}}=\mathrm{S}_{\mathrm{L}} \circ \mathrm{pr}_{1}=\mathrm{pr}_{2}$ because of Lemma 4.14

We need to prove the same for the range map $r_{G}: G^{1} \rightarrow G^{0}$. The inverse map would help us for proving this, so let us construct it firstly.

Consider the arrow

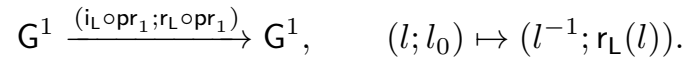

First of all, we have to show that this arrow is well-defined. Let us find out what we need for the arrow $\left(i_{\mathrm{L}} \circ \mathrm{pr}_{1} ; \mathrm{r}_{\mathrm{L}} \circ \mathrm{pr}_{1}\right)$ to be well-defined. We need that the arrows $\left(\mathrm{F}^{1} ; \mathrm{s}_{\mathrm{L}}\right) \circ \mathrm{i}_{\mathrm{L}} \circ \mathrm{pr}_{1}$ and $\left(\mathrm{F}^{1} \circ \mathrm{u}_{\mathrm{L}} ; \mathrm{id}_{\mathrm{L}^{0}}\right) \circ \mathrm{r}_{\mathrm{L}} \circ \mathrm{pr}_{1}$ be equal. The first one is the same as $\left(\mathrm{F}^{1} \circ \mathrm{i}_{\mathrm{L}} \circ \mathrm{pr}_{1} ; \mathrm{s}_{\mathrm{L}} \circ \mathrm{i}_{\mathrm{L}} \circ \mathrm{pr}_{1}\right)$ and the second one is $\left(\mathrm{F}^{1} \circ \mathrm{u}_{\mathrm{L}} \circ \mathrm{r}_{\mathrm{L}} \circ \mathrm{pr}_{1} ; \mathrm{id}_{\mathrm{L}} \circ \circ \mathrm{r}_{\mathrm{L}} \circ \mathrm{pr}_{1}\right)$. It is clear that the right parts of the arrows are equal, $\mathrm{s}_{\mathrm{L}} \circ \mathrm{i}_{\mathrm{L}} \circ \mathrm{pr}_{1}=\mathrm{id}_{\mathrm{L}^{0}} \circ \mathrm{r}_{\mathrm{L}} \circ \mathrm{pr}_{1}$. So we need to prove that the left parts of the arrows are equal too. We have to show that the arrows $\mathrm{F}^{1} \circ \mathrm{i}_{\mathrm{L}} \circ \mathrm{pr}_{1}$ and $\mathrm{F}^{1} \circ \mathrm{u}_{\mathrm{L}} \circ \mathrm{r}_{\mathrm{L}} \circ \mathrm{pr}_{1}$ from $\mathrm{G}^{1}$ to $\mathrm{H}^{1}$ are equal. That is right because we have (3) in Corollary 3.9 and Lemma 4.14 and we can write:

$$
\begin{aligned}
\mathrm{F}^{1}\left(l^{-1}\right) & =\left(\mathrm{F}^{1}(l)\right)^{-1} \\
& =\left(\mathrm{F}^{1}\left(1_{\mathrm{s}_{\mathrm{L}}(l)}\right)\right)^{-1} \\
& =\mathrm{F}^{1}\left(\left(1_{\mathrm{s}_{\mathrm{L}}(l)}\right)^{-1}\right) \\
& =\mathrm{F}^{1}\left(1_{\mathrm{s}_{\mathrm{L}}(l)}\right) \\
& =\mathrm{F}^{1}(l)
\end{aligned}
$$

for all $\left(l ; l_{0}\right) \in \mathrm{G}^{1}$. Therefore, $\mathrm{F}^{1} \circ \mathrm{pr}_{1}=\mathrm{F}^{1} \circ \mathrm{i}_{\mathrm{L}} \circ \mathrm{pr}_{1}$. Hence

$$
\begin{aligned}
\mathrm{F}^{1}\left(1_{\mathrm{r}_{\mathrm{L}}(l)}\right) & =\mathrm{F}^{1}\left(1_{\mathrm{s}_{\mathrm{L}}\left(l^{-1}\right)}\right) \\
& =1_{\mathrm{F}^{0}\left(\mathrm{~s}_{\mathrm{L}}\left(l^{-1}\right)\right)} \\
& =1_{\mathrm{s}_{\mathrm{H}}}\left(\mathrm{F}^{1}\left(l^{-1}\right)\right) \\
& =1_{\mathrm{s}_{\mathrm{H}}\left(\mathrm{F}^{1}(l)\right)} \\
& =1_{\mathrm{F}^{0}\left(\mathrm{~s}_{\mathrm{L}}(l)\right)} \\
& =\mathrm{F}^{1}\left(1_{\mathrm{s}_{\mathrm{L}}(l)}\right) \\
& =\mathrm{F}^{1}(l) \\
& =\mathrm{F}^{1}\left(l^{-1}\right)
\end{aligned}
$$

for all $\left(l ; l_{0}\right) \in \mathrm{G}^{1}$. Therefore, $\left(\mathrm{F}^{1}\left(l^{-1}\right) ; \mathrm{s}_{\mathrm{L}}\left(l^{-1}\right)\right)=\left(\mathrm{F}^{1}\left(1_{\mathrm{r}_{\mathrm{L}}(l)}\right) ; \mathrm{r}_{\mathrm{L}}(l)\right)$ for all $\left(l ; l_{0}\right) \in \mathrm{G}^{1}$. Thus the arrow $\left(\mathrm{i}_{\mathrm{L}} \circ \mathrm{pr}_{1} ; \mathrm{r}_{\mathrm{L}} \circ \mathrm{pr}_{1}\right): \mathrm{G}^{1} \rightarrow \mathrm{G}^{1}$ is well-defined. Denote it by $\mathrm{i}_{\mathrm{G}}$ and 
consider the composition $\mathrm{i}_{\mathrm{G}} \circ \mathrm{i}_{\mathrm{G}}$ :

$$
\begin{aligned}
\mathrm{i}_{\mathrm{G}}\left(\mathrm{i}_{\mathrm{G}}\left(l ; l_{0}\right)\right) & =\mathrm{i}_{\mathrm{G}}\left(l^{-1} ; \mathrm{r}_{\mathrm{L}}(l)\right) \\
& =\left(\left(l^{-1}\right)^{-1} ; \mathrm{r}_{\mathrm{L}}\left(l^{-1}\right)\right) \\
& =\left(l ; \mathrm{s}_{\mathrm{L}}(l)\right) \\
& =\left(l ; l_{0}\right)
\end{aligned}
$$

for all $\left(l ; l_{0}\right) \in \mathrm{G}^{1}$. So $\mathrm{i}_{\mathrm{G}} \circ \mathrm{i}_{\mathrm{G}}=\mathrm{id}_{\mathrm{G}^{1}}$. Hence $\mathrm{i}_{\mathrm{G}}$ is an isomorphism. Also we have

$$
\begin{aligned}
\mathrm{s}_{\mathrm{G}}\left(\mathrm{i}_{\mathrm{G}}\left(l ; l_{0}\right)\right) & =\mathrm{s}_{\mathrm{G}}\left(l^{-1} ; \mathrm{r}_{\mathrm{L}}(l)\right) \\
& =\mathrm{s}_{\mathrm{L}}\left(l^{-1}\right) \\
& =\mathrm{r}_{\mathrm{L}}(l) \\
& =\mathrm{r}_{\mathrm{G}}\left(l ; l_{0}\right)
\end{aligned}
$$

for all $\left(l ; l_{0}\right) \in \mathrm{G}^{1}$. Therefore, $\mathrm{s}_{\mathrm{G}} \circ \mathrm{i}_{\mathrm{G}}=\mathrm{r}_{\mathrm{G}}$. Hence the range map $\mathrm{r}_{\mathrm{G}}: \mathrm{G}^{1} \rightarrow \mathrm{G}^{1}$ is a cover like the source map $\mathrm{s}_{\mathrm{G}}: \mathrm{G}^{1} \rightarrow \mathrm{G}^{1}$. So the condition (1) in Definition 3.1 is satisfied. Now let us prove the condition $(2)$.

$$
\begin{aligned}
\mathrm{r}_{\mathrm{G}}\left(\left(l ; l_{0}\right) \cdot\left(l^{\prime} ; l_{0}^{\prime}\right)\right) & =\mathrm{r}_{\mathrm{G}}\left(l \cdot l^{\prime} ; l_{0}^{\prime}\right) \\
& =\mathrm{r}_{\mathrm{L}}\left(l \cdot l^{\prime}\right) \\
& =\mathrm{r}_{\mathrm{L}}(l)=\mathrm{r}_{\mathrm{G}}\left(\left(l ; l_{0}\right)\right)
\end{aligned}
$$

and

$$
\begin{aligned}
\mathrm{s}_{\mathrm{G}}\left(\left(l ; l_{0}\right) \cdot\left(l^{\prime} ; l_{0}^{\prime}\right)\right) & =\mathrm{s}_{\mathrm{G}}\left(l \cdot l^{\prime} ; l_{0}^{\prime}\right) \\
& =\mathrm{s}_{\mathrm{L}}\left(l \cdot l^{\prime}\right) \\
& =\mathrm{s}_{\mathrm{L}}\left(l^{\prime}\right) \\
& =\mathrm{s}_{\mathrm{G}}\left(\left(l^{\prime} ; l_{0}^{\prime}\right)\right)
\end{aligned}
$$

for all $\left(l ; l_{0}\right),\left(l^{\prime} ; l_{0}^{\prime}\right) \in \mathrm{G}^{1}$ with $\mathrm{s}_{\mathrm{G}}\left(l ; l_{0}\right)=\mathrm{r}_{\mathrm{G}}\left(l^{\prime} ; l_{0}^{\prime}\right)$. So the condition $(2)$ is satisfied.

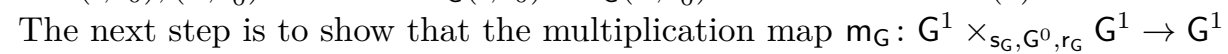
is associative. We have

$$
\begin{aligned}
\left(\left(l ; l_{0}\right) \cdot\left(l^{\prime} ; l_{0}^{\prime}\right)\right) \cdot\left(l^{\prime \prime} ; l_{0}^{\prime \prime}\right) & =\left(l \cdot l^{\prime} ; l_{0}^{\prime}\right) \cdot\left(l^{\prime \prime} ; l_{0}^{\prime \prime}\right) \\
& =\left(\left(l \cdot l^{\prime}\right) \cdot l^{\prime \prime} ; l_{0}^{\prime \prime}\right) \\
& =\left(l \cdot\left(l^{\prime} \cdot l^{\prime \prime}\right) ; l_{0}^{\prime \prime}\right) \\
& =\left(l ; l_{0}\right) \cdot\left(\left(l^{\prime} ; l_{0}^{\prime}\right) \cdot\left(l^{\prime \prime} ; l_{0}^{\prime \prime}\right)\right)
\end{aligned}
$$

for all $\left(l ; l_{0}\right),\left(l^{\prime} ; l_{0}^{\prime}\right),\left(l^{\prime \prime} ; l_{0}^{\prime \prime}\right) \in \mathrm{G}^{1}$ with $\mathrm{s}_{\mathrm{G}}\left(l ; l_{0}\right)=\mathrm{r}_{\mathrm{G}}\left(l^{\prime} ; l_{0}^{\prime}\right)$ and $\mathrm{s}_{\mathrm{G}}\left(l^{\prime} ; l_{0}^{\prime}\right)=\mathrm{r}_{\mathrm{G}}\left(l^{\prime \prime} ; l_{0}^{\prime \prime}\right)$. So the multiplication map $\mathrm{m}_{\mathrm{G}}$ is associative.

We have proved the properties (1) (2) and (3) in Definition 3.1. The next step is to construct the arrow $\mathrm{u}_{\mathrm{G}}: \mathrm{G}^{0} \rightarrow \mathrm{G}^{1}$ and prove all properties in Proposition 3.8 . which then allows us to use Proposition 3.11

Consider the arrow

$$
\mathrm{G}^{0} \stackrel{\left(\mathrm{u}_{\mathrm{L}} ; \mathrm{id}_{\mathrm{L} 0}\right)}{\longrightarrow} \mathrm{G}^{1}, \quad l_{0} \mapsto\left(1_{l_{0}} ; l_{0}\right)
$$

for all $l_{0} \in \mathrm{L}^{0}$. It is well-defined because $\left(\mathrm{F}^{1}\left(1_{l_{0}}\right) ; \mathrm{s}_{\mathrm{L}}\left(1_{l_{0}}\right)\right)=\left(\mathrm{F}^{1}\left(1_{l_{0}}\right) ; l_{0}\right)$ for all $l_{0} \in \mathrm{L}^{0}$. Denote it by $\mathrm{u}_{\mathrm{G}}$. The property (1) in Proposition 3.8 is clear:

$$
\begin{aligned}
\mathrm{s}_{\mathrm{G}}\left(\mathrm{u}_{\mathrm{G}}\left(l_{0}\right)\right) & =\mathrm{s}_{\mathrm{G}}\left(1_{l_{0}} ; l_{0}\right) \\
& =\mathrm{s}_{\mathrm{L}}\left(1_{l_{0}}\right) \\
& =l_{0}
\end{aligned}
$$


and

$$
\begin{aligned}
\mathrm{r}_{\mathrm{G}}\left(\mathrm{u}_{\mathrm{G}}\left(l_{0}\right)\right) & =\mathrm{r}_{\mathrm{G}}\left(1_{l_{0}} ; l_{0}\right) \\
& =\mathrm{r}_{\mathrm{L}}\left(1_{l_{0}}\right) \\
& =l_{0}
\end{aligned}
$$

for all $l_{0} \in \mathrm{L}^{0}$. Also, we have

$$
\begin{aligned}
\mathrm{u}_{\mathrm{G}}\left(\mathrm{r}_{\mathrm{G}}\left(l ; l_{0}\right)\right) \cdot\left(l ; l_{0}\right) & =\mathrm{u}_{\mathrm{G}}\left(\mathrm{r}_{\mathrm{L}}(l)\right) \cdot\left(l ; l_{0}\right) \\
& =\left(1_{\mathrm{r}_{\mathrm{L}}(l)} ; \mathrm{r}_{\mathrm{L}}(l)\right) \cdot\left(l ; l_{0}\right) \\
& =\left(1_{\mathrm{r}_{\mathrm{L}}(l)} \cdot l ; l_{0}\right) \\
& =\left(l ; l_{0}\right)
\end{aligned}
$$

and

$$
\begin{aligned}
\left(l ; l_{0}\right) \cdot \mathrm{u}_{\mathrm{G}}\left(\mathrm{s}_{\mathrm{G}}\left(l ; l_{0}\right)\right) & =\left(l ; l_{0}\right) \cdot \mathrm{u}_{\mathrm{G}}\left(\mathrm{s}_{\mathrm{L}}(l)\right) \\
& =\left(l ; l_{0}\right) \cdot\left(1_{\mathrm{s}_{\mathrm{L}}(l)} ; \mathrm{s}_{\mathrm{L}}(l)\right) \\
& =\left(l \cdot 1_{\mathrm{s}_{\mathrm{L}}(l)} ; \mathrm{s}_{\mathrm{L}}(l)\right) \\
& =\left(l ; l_{0}\right)
\end{aligned}
$$

for all $\left(l ; l_{0}\right) \in \mathrm{G}^{1}$. So the property $(2)$ is proved.

We proved above that $\mathrm{r}_{\mathrm{G}}=\mathrm{s}_{\mathrm{G}} \circ \mathrm{i}_{\mathrm{G}}$ and $\mathrm{i}_{\mathrm{G}} \circ \mathrm{i}_{\mathrm{G}}=\mathrm{id}_{\mathrm{G}^{1}}$, thus $\mathrm{s}_{\mathrm{G}}=\mathrm{s}_{\mathrm{G}} \circ \mathrm{i}_{\mathrm{G}} \circ \mathrm{i}_{\mathrm{G}}=\mathrm{r}_{\mathrm{G}} \circ \mathrm{i}_{\mathrm{G}}$. So property (3) is done.

The last step is to prove the property (4) in Proposition 3.8. We have

$$
\begin{aligned}
\mathrm{i}_{\mathrm{G}}\left(l ; l_{0}\right) \cdot\left(l ; l_{0}\right) & =\left(l^{-1} ; \mathrm{r}_{\mathrm{L}}(l)\right) \cdot\left(l ; l_{0}\right) \\
& =\left(l^{-1} \cdot l ; l_{0}\right) \\
& =\left(1_{\mathrm{s}_{\mathrm{L}}(l)} ; \mathrm{s}_{\mathrm{L}}(l)\right) \\
& =\left(1_{\mathrm{s}_{\mathrm{G}}\left(l ; l_{0}\right)} ; \mathrm{s}_{\mathrm{G}}\left(l ; l_{0}\right)\right) \\
& =\mathrm{u}_{\mathrm{G}}\left(\mathrm{s}_{\mathrm{G}}\left(l ; l_{0}\right)\right)
\end{aligned}
$$

and

$$
\begin{aligned}
\left(l ; l_{0}\right) \cdot \mathrm{i}_{\mathrm{G}}\left(l ; l_{0}\right) & =\left(l ; l_{0}\right) \cdot\left(l^{-1} ; \mathrm{r}_{\mathrm{L}}(l)\right) \\
& =\left(l \cdot l^{-1} ; \mathrm{r}_{\mathrm{L}}(l)\right) \\
& =\left(1_{\mathrm{r}_{\mathrm{L}}(l)} ; \mathrm{r}_{\mathrm{L}}(l)\right) \\
& =\left(1_{\mathrm{r}_{\mathrm{G}}\left(l ; l_{0}\right)} ; \mathrm{r}_{\mathrm{G}}\left(l ; l_{0}\right)\right) \\
& =\mathrm{u}_{\mathrm{G}}\left(\mathrm{r}_{\mathrm{G}}\left(l ; l_{0}\right)\right)
\end{aligned}
$$

for all $\left(l ; l_{0}\right) \in \mathrm{G}^{1}$. So we have all required properties in Proposition 3.11 Therefore, $\mathrm{G}=\left(\mathrm{G}^{0}, \mathrm{G}^{1}, \mathrm{r}_{\mathrm{G}}, \mathrm{s}_{\mathrm{G}}, \mathrm{m}_{\mathrm{G}}\right)$ is a groupoid.

Remark 4.16. We shortly denote the element $\left(l ; l_{0}\right) \in \mathrm{G}^{1}$ by $g$ and we always mean an element $g \in \mathrm{G}^{1}$ is equivalent to $g \in \mathrm{L}^{1}$ and with $\mathrm{F}^{1}(g)=\mathrm{F}^{1}\left(1_{\mathrm{S}_{\mathrm{L}}(g)}\right)$.

Remark 4.17. Let $G$ be the fibre of the partial groupoid fibration $F: L \rightarrow H$. The pair $\left(\mathrm{pr}_{1} ; \mathrm{id}_{\mathrm{L} 0}\right)$ defines a functor from $\mathrm{G}$ to $\mathrm{L}$. It intertwines the source, range and multiplication maps by definition. Let us call this functor an inclusion. This functor is always monic. Here $\mathrm{I}$ mean that the arrow $\mathrm{pr}_{1}: \mathrm{G}^{1} \longmapsto \mathrm{L}^{1}$ is monic. That is true because if for some parallel pair of arrows $x_{1}, x_{2}: \mathrm{X} \rightrightarrows \mathrm{G}^{1}$ we have $\mathrm{pr}_{1} \circ x_{1}=\mathrm{pr}_{1} \circ x_{2}$ then $\mathrm{pr}_{2} \circ x_{1}=\mathrm{s}_{\mathrm{L}} \circ \mathrm{pr}_{1} \circ x_{1}=\mathrm{s}_{\mathrm{L}} \circ \mathrm{pr}_{1} \circ x_{2}=\mathrm{pr}_{2} \circ x_{2}$. Therefore, $x_{1}=x_{2}$ by universal property of the fibre product. Hence $\mathrm{pr}_{1}: \mathrm{G}^{1} \longmapsto \mathrm{L}^{1}$ is monic.

We will use the notation $\mathrm{G} \hookrightarrow \mathrm{L} \rightarrow \mathrm{H}$ to denote that we have a partial groupoid fibration from $\mathrm{L}$ to $\mathrm{H}$ with fibre $\mathrm{G}$. 
Example 4.18. Any functor $\mathrm{F}$ from a groupoid $\mathrm{L}$ to a 0 -groupoid $\mathrm{H}$ is a groupoid fibration with fibre isomorphic to $L$. Here the source map $\mathrm{s}_{\mathrm{H}}: \mathrm{H}^{1} \rightarrow \mathrm{H}^{0}$ is an

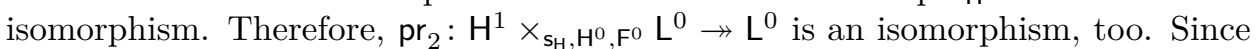
$\left(\mathrm{F}^{1} ; \mathrm{s}_{\mathrm{L}}\right)=\left(\mathrm{pr}_{2}\right)^{-1} \circ \mathrm{s}_{\mathrm{L}}$, the arrow $\left(\mathrm{F}^{1} ; \mathrm{s}_{\mathrm{L}}\right)$ is a partial cover. Moreover, it splits by $\mathrm{u}_{\mathrm{L}} \circ \mathrm{pr}_{2}$. Thus it is a cover. Hence $\mathrm{F}$ is a groupoid fibration. Since $\mathrm{pr}_{2} \circ\left(\mathrm{F}^{1} \circ \mathrm{u}_{\mathrm{L}} ; \mathrm{id}_{\mathrm{L}^{0}}\right)=$ $\mathrm{id}_{\mathrm{L}^{0}}$, we have $\left(\mathrm{pr}_{2}\right)^{-1}=\left(\mathrm{F}^{1} \circ \mathrm{u}_{\mathrm{L}} ; \mathrm{id}_{\mathrm{L}^{0}}\right)$. Therefore, $\left(\mathrm{F}^{1} \circ \mathrm{u}_{\mathrm{L}} ; \mathrm{id}_{\mathrm{L}^{0}}\right)$ is an isomorphism. Hence its pull-back is an isomorphism, too, and the functor described in Remark 4.17 is an identity on objects and an isomorphism on arrows. Therefore, the fibre of $\mathrm{F}$ is isomorphic to $\mathrm{L}$.

Lemma 4.19. The fibre of a partial groupoid fibration $\mathrm{F}: \mathrm{L} \rightarrow \mathrm{H}$ is a 0 -groupoid if and only if $\mathrm{F}$ is a partial groupoid covering.

Proof. Suppose that $\mathrm{F}: \mathrm{L} \rightarrow \mathrm{H}$ is a partial groupoid covering. That is, the arrow

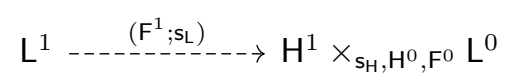

is a partial cover and monic. We are going to prove that $\mathrm{u}_{\mathrm{G}} \circ \mathrm{s}_{\mathrm{G}}=\mathrm{id}_{\mathrm{G}^{1}}$, where $\mathrm{G}$ is the fibre of $\mathrm{F}$. We have

$$
\begin{aligned}
\left(\mathrm{F}^{1} ; \mathrm{s}_{\mathrm{L}}\right)(g) & =\left(\mathrm{F}^{1}(g) ; \mathrm{s}_{\mathrm{L}}(g)\right) \\
& =\left(1_{\mathrm{s}_{\mathrm{L}}(g)} ; \mathrm{s}_{\mathrm{L}}(g)\right) \\
& =\left(1_{\mathrm{s}_{\mathrm{L}}(g)} ; \mathrm{s}_{\mathrm{L}}\left(1_{\mathrm{S}_{\mathrm{L}}(g)}\right)\right) \\
& =\left(\mathrm{F}^{1}\left(1_{\mathrm{S}_{\mathrm{L}}(g)}\right) ; \mathrm{s}_{\mathrm{L}}\left(1_{\mathrm{S}_{\mathrm{L}}(g)}\right)\right) \\
& =\left(\mathrm{F}^{1} ; \mathrm{s}_{\mathrm{L}}\right)\left(1_{\mathrm{S}_{\mathrm{L}}(g)}\right)
\end{aligned}
$$

for all $g \in \mathrm{G}^{1}$. Since $\left(\mathrm{F}^{1} ; \mathrm{s}_{\mathrm{L}}\right)$ is monic, $1_{\mathrm{s}_{\mathrm{L}}(g)}=g$ for all $g \in \mathrm{G}^{1}$. Therefore, $\mathrm{u}_{\mathrm{G}} \circ \mathrm{s}_{\mathrm{G}}=\mathrm{id}_{\mathrm{G}^{1}}$. Also we know that $\mathrm{s}_{\mathrm{G}} \circ \mathrm{u}_{\mathrm{G}}=\mathrm{id}_{\mathrm{G}^{0}}$. Hence the source map of $\mathrm{G}$ is an isomorphism. Therefore, $\mathrm{G}$ is a 0 -groupoid by Lemma 4.4

Conversely, suppose that $\mathrm{G}$ is a 0 -groupoid. That is, the source map $\mathrm{s}_{\mathrm{G}}$ is an isomorphism. Suppose that there are elements $l_{1}, l_{2} \in \mathrm{L}^{1}$ such that $\left(\mathrm{F}^{1} ; \mathrm{s}_{\mathrm{L}}\right)\left(l_{1}\right)=$ $\left(\mathrm{F}^{1} ; \mathrm{s}_{\mathrm{L}}\right)\left(l_{2}\right)$. Hence $\left(\mathrm{F}^{1}\left(l_{1}\right) ; \mathrm{s}_{\mathrm{L}}\left(l_{1}\right)\right)=\left(\mathrm{F}^{1}\left(l_{2}\right) ; \mathrm{s}_{\mathrm{L}}\left(l_{2}\right)\right)$. Thus $\mathrm{F}^{1}\left(l_{1}\right)=\mathrm{F}^{1}\left(l_{2}\right)$ and $\mathrm{s}_{\mathrm{L}}\left(l_{1}\right)=$

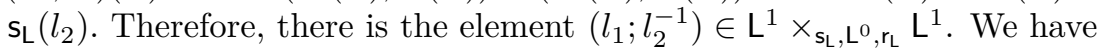

$$
\begin{aligned}
\mathrm{F}^{1}\left(l_{1} \cdot l_{2}^{-1}\right) & =\mathrm{F}^{1}\left(l_{1}\right) \cdot \mathrm{F}^{1}\left(l_{2}^{-1}\right) \\
& =\mathrm{F}^{1}\left(l_{2}\right) \cdot \mathrm{F}^{1}\left(l_{2}\right)^{-1} \\
& =1_{\mathrm{r}_{\mathrm{H}}\left(\mathrm{F}^{1}\left(l_{2}\right)\right)} \\
& =1_{\mathrm{F}^{0}\left(\mathrm{r}_{\mathrm{L}}\left(l_{2}\right)\right)} .
\end{aligned}
$$

Therefore, the element $l_{1} \cdot l_{2}^{-1}$ is in G. Also, the element $l_{2} \cdot l_{2}^{-1}$ is in G. We have $\mathrm{s}_{\mathrm{G}}\left(l_{1} \cdot l_{2}^{-1}\right)=\mathrm{s}_{\mathrm{G}}\left(l_{2}^{-1}\right)=\mathrm{s}_{\mathrm{G}}\left(l_{2} \cdot l_{2}^{-1}\right)$. We know that $\mathrm{s}_{\mathrm{G}}$ is an isomorphism. Thus $l_{1}=l_{2}$. Hence $\left(\mathrm{F}^{1} ; \mathrm{s} \mathrm{L}\right)$ is monic and, therefore, $\mathrm{F}: \mathrm{L} \rightarrow \mathrm{H}$ is a partial groupoid covering.

Corollary 4.20. The fibre of a groupoid fibration $\mathrm{F}: \mathrm{L} \rightarrow \mathrm{H}$ is a 0 -groupoid if and only if $\mathrm{F}$ is a groupoid covering.

Proof. If $\mathrm{F}: \mathrm{L} \rightarrow \mathrm{H}$ is a groupoid covering then it is a partial groupoid covering by Lemma 4.19. Therefore, the fibre of $\mathrm{F}: \mathrm{L} \rightarrow \mathrm{H}$ is a 0 -groupoid.

Conversely, if the fibre of the groupoid fibration $\mathrm{F}: \mathrm{L} \rightarrow \mathrm{H}$ is a 0 -groupoid, then $\mathrm{F}$ is a partial groupoid covering by Lemma 4.19 So $\mathrm{F}$ is a groupoid fibration and a partial groupoid covering. Therefore, it is a groupoid covering by Remark 4.8

Lemma 4.21. The groupoid fibration described in Example 4.18 is a groupoid covering if and only if $\mathrm{L}$ is a 0-groupoid. 
Proof. If $\mathrm{L}$ is a 0 -groupoid, then the fibre of $\mathrm{F}$ is a 0 -groupoid, too. Therefore, $\mathrm{F}$ is a groupoid covering by Corollary 4.20 Conversely, if $\mathrm{F}$ is a groupoid covering, then

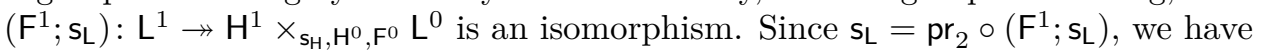
that $S_{\mathrm{L}}$ is an isomorphism, too. Therefore, $\mathrm{L}$ is a 0 -groupoid.

Proposition 4.22. Let $\mathrm{F}: \mathrm{L} \rightarrow \mathrm{H}$ and $\mathrm{E}: \mathrm{H} \rightarrow \mathrm{R}$ be a composable pair of partial groupoid fibrations with fibres $\mathrm{G}_{1}$ and $\mathrm{G}_{2}$, respectively. Let $\mathrm{G}$ be the fibre of their composition. There is a partial groupoid fibration $\left.\mathrm{F}\right|_{\mathrm{G}}: \mathrm{G} \rightarrow \mathrm{G}_{2}$ which commutes with inclusions and $\mathrm{F}$, and its fibre is isomorphic to $\mathrm{G}_{1}$, as in the diagram

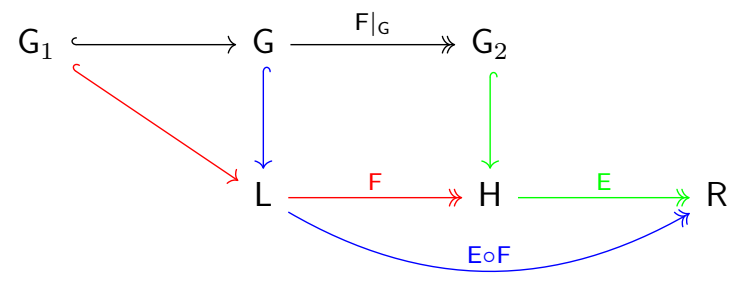

Proof. Firstly, we construct the functor $\left.F\right|_{G}$ from $G$ to $G_{2}$. The objects of these groupoids are $\mathrm{L}^{0}$ and $\mathrm{H}^{0}$. Let $\left.\mathrm{F}\right|_{\mathrm{G}}$ be $\mathrm{F}^{0}$ on objects and let $\left.\mathrm{F}\right|_{\mathrm{G}} ^{1}=\left(\mathrm{F}^{1} \circ \mathrm{pr}_{1} ; \mathrm{F}^{0} \circ \mathrm{pr}_{2}\right)$. It must be well-defined. We have

$$
\begin{aligned}
\mathrm{E}^{1}\left(\mathrm{~F}^{1}(g)\right) & =\mathrm{E}^{1}\left(\mathrm{~F}^{1}\left(1_{\mathrm{S}_{\mathrm{L}}(g)}\right)\right) \\
& =\mathrm{E}^{1}\left(1_{\mathrm{F}^{0}\left(\mathrm{sL}_{\mathrm{L}}(g)\right)}\right) \\
& =\mathrm{E}^{1}\left(1_{\mathrm{SH}_{\mathrm{H}}\left(\mathrm{F}^{1}(g)\right)}\right)
\end{aligned}
$$

for all $g \in \mathrm{G}^{1}$. Therefore, $\mathrm{F}^{1}(g) \in \mathrm{G}_{2}^{1}$. Now, we need to prove that $\left.\mathrm{F}\right|_{\mathrm{G}}$ intertwines the source, range and multiplication maps of $G$ and $G_{2}$. There is an important commutation which holds between arrows of $G, G_{2}$, $L$ and $H$. That is

$$
\begin{aligned}
\left.\mathrm{pr}_{1} \circ \mathrm{F}\right|_{\mathrm{G}} ^{1} & =\mathrm{pr}_{1} \circ\left(\mathrm{F}^{1} \circ \mathrm{pr}_{1} ; \mathrm{F}^{0} \circ \mathrm{pr}_{2}\right) \\
& =\mathrm{F}^{1} \circ \mathrm{pr}_{1} .
\end{aligned}
$$

Therefore, we have

$$
\begin{aligned}
\mathrm{F}^{0} \circ \mathrm{r}_{\mathrm{G}} & =\mathrm{F}^{0} \circ \mathrm{r}_{\mathrm{L}} \circ \mathrm{pr}_{1} \\
& =\mathrm{r}_{\mathrm{H}} \circ \mathrm{F}^{1} \circ \mathrm{pr}_{1} \\
& =\left.\mathrm{r}_{\mathrm{H}} \circ \mathrm{pr}_{1} \circ \mathrm{F}\right|_{\mathrm{G}} ^{1} \\
& =\left.\mathrm{r}_{\mathrm{G}_{2}} \circ \mathrm{F}\right|_{\mathrm{G}} ^{1} .
\end{aligned}
$$

Analogously, $\left.\mathrm{F}\right|_{\mathrm{G}}$ intertwines the source maps: $\mathrm{F}^{0} \circ \mathrm{s}_{\mathrm{G}}=\left.\mathrm{s}_{\mathrm{G}_{2}} \circ \mathrm{F}\right|_{\mathrm{G}} ^{1}$.

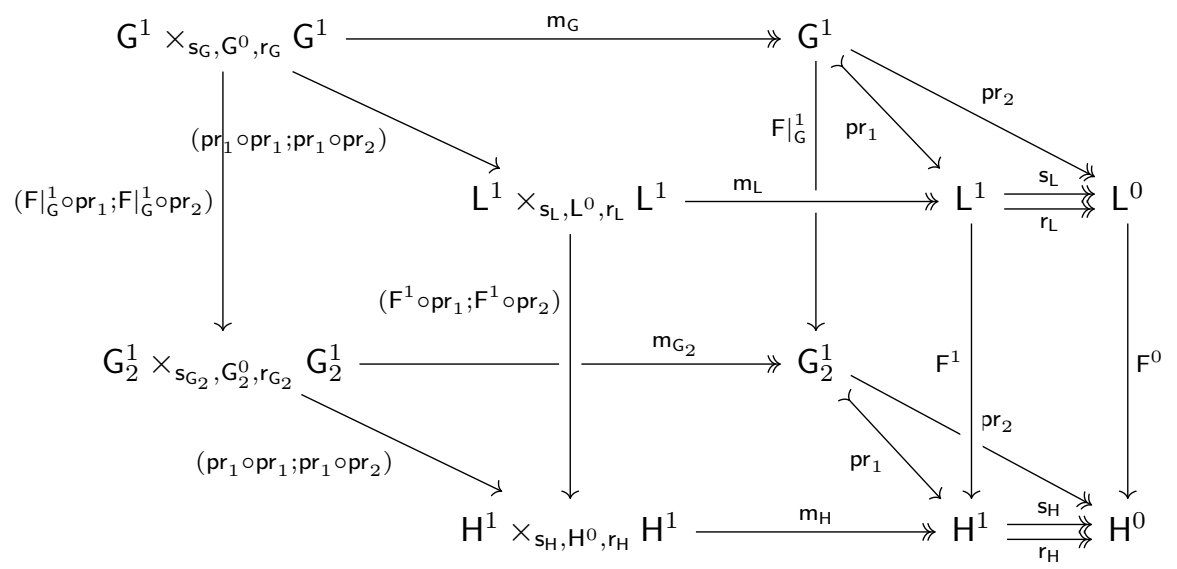


It is easy to check that every relevant square commutes in the diagram. The up and bottom squares commutes because of the definition of the multiplication map of the fibre. The left square commutes because the right one does. The square in front of the reader commutes because the functor $F$ intertwines the multiplication maps of $L$ and $H$. Finally, we have

$$
\begin{aligned}
\left.\mathrm{pr}_{1} \circ \mathrm{F}\right|_{\mathrm{G}} ^{1} \circ \mathrm{m}_{\mathrm{G}} & =\mathrm{F}^{1} \circ \mathrm{pr}_{1} \circ \mathrm{m}_{\mathrm{G}} \\
& =\mathrm{F}^{1} \circ \mathrm{m}_{\mathrm{L}} \circ\left(\mathrm{pr}_{1} \circ \mathrm{pr}_{1} ; \mathrm{pr}_{1} \circ \mathrm{pr}_{2}\right) \\
& =\mathrm{m}_{\mathrm{H}} \circ\left(\mathrm{F}^{1} \circ \mathrm{pr}_{1} ; \mathrm{F}^{1} \circ \mathrm{pr}_{2}\right) \circ\left(\mathrm{pr}_{1} \circ \mathrm{pr}_{1} ; \mathrm{pr}_{1} \circ \mathrm{pr}_{2}\right) \\
& =\mathrm{m}_{\mathrm{H}} \circ\left(\mathrm{pr}_{1} \circ \mathrm{pr}_{1} ; \mathrm{pr}_{1} \circ \mathrm{pr}_{2}\right) \circ\left(\left.\mathrm{F}\right|_{\mathrm{G}} ^{1} \circ \mathrm{pr}_{1} ;\left.\mathrm{F}\right|_{\mathrm{G}} ^{1} \circ \mathrm{pr}_{2}\right) \\
& =\mathrm{pr}_{1} \circ \mathrm{m}_{\mathrm{G}_{2}} \circ\left(\left.\mathrm{F}\right|_{\mathrm{G}} ^{1} \circ \mathrm{pr}_{1} ;\left.\mathrm{F}\right|_{\mathrm{G}} ^{1} \circ \mathrm{pr}_{2}\right) .
\end{aligned}
$$

We know from Remark 4.17 that $\mathrm{pr}_{1}: \mathrm{G}_{2}^{1} \longmapsto \mathrm{H}^{1}$ is monic. Therefore, we have $\left.\mathrm{F}\right|_{\mathrm{G}} ^{1} \circ \mathrm{m}_{\mathrm{G}}=\mathrm{m}_{\mathrm{G}_{2}} \circ\left(\left.\mathrm{F}\right|_{\mathrm{G}} ^{1} \circ \mathrm{pr}_{1} ;\left.\mathrm{F}\right|_{\mathrm{G}} ^{1} \circ \mathrm{pr}_{2}\right)$. Hence $\left.\mathrm{F}\right|_{\mathrm{G}}$ intertwines the multiplication maps, too.

Now, we have to show that this well-defined functor $\left.F\right|_{G}: G \rightarrow G_{2}$ is a partial groupoid fibration. First step is to show that the following diagram

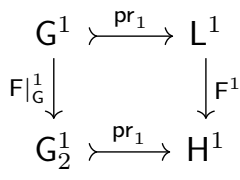

is a pull-back square. Suppose that there are arrows $x_{1}: \mathrm{X} \rightarrow \mathrm{L}^{1}$ and $x_{2}: \mathrm{X} \rightarrow \mathrm{G}_{2}^{1}$ such that $\mathrm{F}^{1} \circ x_{1}=\mathrm{pr}_{1} \circ x_{2}$. Hence

$$
\begin{aligned}
\mathrm{E}^{1} \circ \mathrm{F}^{1} \circ x_{1} & =\mathrm{E}^{1} \circ \mathrm{pr}_{1} \circ x_{2} \\
& =\mathrm{E}^{1} \circ \mathrm{u}_{\mathrm{H}} \circ \mathrm{s}_{\mathrm{H}} \circ \mathrm{pr}_{1} \circ x_{2} \\
& =\mathrm{E}^{1} \circ \mathrm{u}_{\mathrm{H}} \circ \mathrm{s}_{\mathrm{H}} \circ \mathrm{F}^{1} \circ x_{1}
\end{aligned}
$$

by Lemma 4.14 Therefore, we have a well-defined arrow $\left(x_{1} ; \mathrm{s}_{\mathrm{L}} \circ x_{1}\right): \mathrm{X} \rightarrow \mathrm{G}^{1}$ because

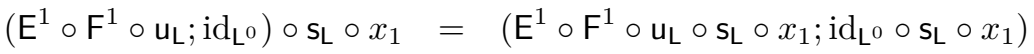

$$
\begin{aligned}
& =\left(\mathrm{E}^{1} \circ \mathrm{u}_{\mathrm{H}} \circ \mathrm{s}_{\mathrm{H}} \circ \mathrm{F}^{1} \circ x_{1} ; \mathrm{s}_{\mathrm{L}} \circ x_{1}\right) \\
& =\left(\mathrm{E}^{1} \circ \mathrm{F}^{1} \circ x_{1} ; \mathrm{s}_{\mathrm{L}} \circ x_{1}\right) \\
& =\left(\mathrm{E}^{1} \circ \mathrm{F}^{1} ; \mathrm{S}_{\mathrm{L}}\right) \circ x_{1} \text {. }
\end{aligned}
$$

Also, $\operatorname{pr}_{1} \circ\left(x_{1} ; \mathrm{s}_{\mathrm{L}} \circ x_{2}\right)=x_{1}$ and

$$
\begin{aligned}
\left.\mathrm{pr}_{1} \circ \mathrm{F}\right|_{\mathrm{G}} ^{1} \circ\left(x_{1} ; \mathrm{s}_{\mathrm{L}} \circ x_{1}\right) & =\mathrm{F}^{1} \circ \mathrm{pr}_{1} \circ\left(x_{1} ; \mathrm{s}_{\mathrm{L}} \circ x_{1}\right) \\
& =\mathrm{F}^{1} \circ x_{1}=\mathrm{pr}_{1} \circ x_{2} .
\end{aligned}
$$

Since $\mathrm{pr}_{1}$ is monic, $\left.\mathrm{F}\right|_{\mathrm{G}} ^{1} \circ\left(x_{1} ; \mathrm{s}_{\mathrm{L}} \circ x_{1}\right)=x_{2}$. Hence the arrow $\left(x_{1} ; \mathrm{s}_{\mathrm{L}} \circ x_{1}\right)$ commutes with $\left.F\right|_{G} ^{1}: G^{1} \rightarrow G_{2}^{1}$ and $\mathrm{pr}_{1}: \mathrm{G}^{1} \longmapsto \mathrm{L}^{1}$. Such an arrow is unique because $\mathrm{pr}_{1}: \mathrm{G}^{1} \longmapsto \mathrm{L}^{1}$ is monic. So the diagram above is a pull-back square. 
Now consider the following diagram:

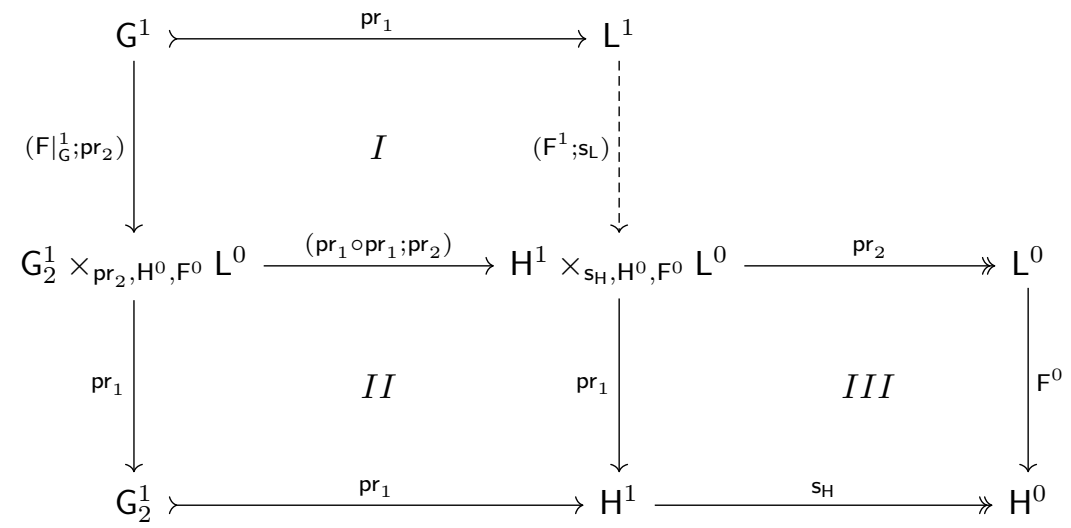

Since $I I I$ and $(I I ; I I I)$ are pull-back squares, $I I$ is a pull-back square. We have shown above that $(I ; I I)$ is a pull-back square, thus $\mathrm{I}$ is a pull-back square, too.

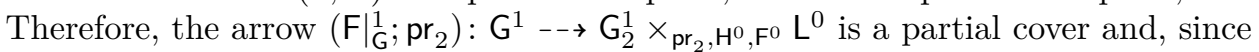
$\mathrm{pr}_{2}=\mathrm{s}_{\mathrm{L}} \circ \mathrm{pr}_{1}=\mathrm{s}_{\mathrm{G}}$, that is why the functor $\left.\mathrm{F}\right|_{\mathrm{G}}: \mathrm{G} \rightarrow \mathrm{G}_{2}$ is a partial groupoid fibration.

The pull-back square $I$ allows to say that the arrows of the fibre of the partial groupoid fibration $\left.F\right|_{G}: G \rightarrow G_{2}$ is isomorphic to $G_{1}^{1}$ because there is a diagram of pull-back squares

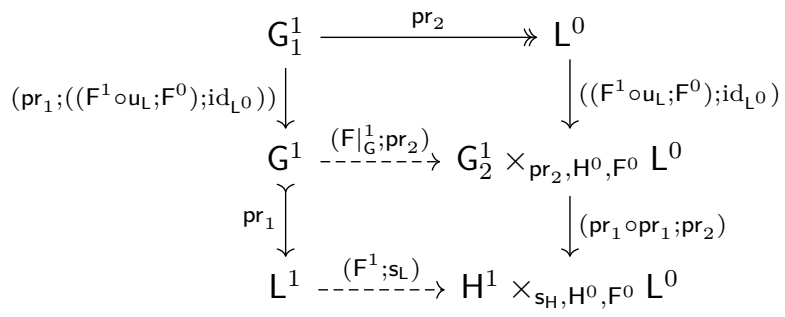

which shows that there is a canonical isomorphism $\left(\mathrm{pr}_{1} \circ \mathrm{pr}_{1} ; \mathrm{pr}_{1}\right)$ between arrows of the fibres of $\left.F\right|_{G}$ and $F$. This isomorphism is natural. The objects of these groupoids are the same, thus they are isomorphic.

Corollary 4.23. Assume all data from Proposition 4.22, Then $\mathrm{F}: \mathrm{L} \rightarrow \mathrm{H}$ is a partial groupoid covering if and only if $\left.\mathrm{F}\right|_{\mathrm{G}}$ is so. If $\mathrm{E}: \mathrm{H} \rightarrow \mathrm{R}$ is a partial groupoid covering, then $\mathrm{G}$ and $\mathrm{G}_{1}$ are isomorphic.

Proof. $\mathrm{F}$ is a partial groupoid covering if and only if $\mathrm{G}_{1}$ is a 0 -groupoid. That is equivalent to $\left.\mathrm{F}\right|_{\mathrm{G}}$ being a partial groupoid covering by Lemma 4.19

If $E$ is a partial groupoid covering, then $G_{2}$ is a 0 -groupoid. Therefore, $G$ and $G_{1}$ are isomorphic by Example 4.18 .

Remark 4.24. Under Assumption 2.15 Proposition 4.22 and Corollary 4.23 are true in global cases instead of partial situations. The proofs are similar ([10], Propositions 2.10 and 2.21).

Example 4.25. Let $f: \mathrm{X} \rightarrow \rightarrow \mathrm{G}^{0}$ be a partial cover over the objects of the groupoid $\mathrm{G}$. The functor $\left(\mathrm{pr}_{1} \circ \mathrm{pr}_{2} ; f\right)$ from the groupoid $\mathrm{G}(\mathrm{X}, f)$, defined in Example 3.16, to $\mathrm{G}$ is a partial groupoid fibration. We just need to check that the arrow (4.1) is a partial cover. It is equal to

$$
\mathrm{pr}_{2}:\left(\mathrm{X} \times_{f, \mathrm{G}^{0}, \mathrm{r}} \mathrm{G}^{1}\right) \times_{\mathrm{pr}_{2}, \mathrm{G}_{1}, \mathrm{pr}_{1}}\left(\mathrm{G}^{1} \times_{\mathrm{s}, \mathrm{G}^{0}, f} \mathrm{X}\right) \rightarrow\left(\mathrm{G}^{1} \times_{\mathrm{s}, \mathrm{G}^{0}, f} \mathrm{X}\right)
$$


and it is a partial cover because it is a pull-back of $\mathrm{pr}_{2}: X \times_{f, \mathrm{G}^{0}, \mathrm{r}} \mathrm{G}^{1} \rightarrow-\mathrm{G}^{1}$, which is a partial cover because it is a pull-back of $f$. It is easy to check that the arrow $\left(\mathrm{pr}_{1} \circ \mathrm{pr}_{1} ; \mathrm{pr}_{2} \circ \mathrm{pr}_{2}\right) \circ \mathrm{pr}_{1}$ is an isomorphism between the arrows of the fibre and the arrows of the Cech groupoid of $f$. Since they have the same objects, the fibre and the Čech groupoid of $f$ are isomorphic. Under Assumption 2.15, we can deduce that if $f: X \rightarrow G^{0}$ is a cover, then this functor is a groupoid fibration.

\section{GROUPOID ACTIONS}

Definition 5.1. Let $\mathrm{G}=\left(\mathrm{G}^{0}, \mathrm{G}^{1}, \mathrm{r}, \mathrm{s}, \mathrm{m}, \mathrm{u}, \mathrm{i}\right)$ be a groupoid. A right $\mathrm{G}$-action $\left(X ; m_{X} ; s_{X}\right)$ is an object $X$ with arrows $s_{X}: X \rightarrow G^{0}$ and $m_{X}: X \times_{s_{X}, G^{0}, r} G^{1} \rightarrow X$ such that

(1) $\mathrm{s}_{\mathrm{X}} \circ \mathrm{m}_{\mathrm{X}}=\mathrm{s} \circ \mathrm{pr}_{2}, \quad \mathrm{sX}_{\mathrm{X}}(x \cdot g)=\mathrm{s}(g)$ for all $x \in \mathrm{X}, g \in \mathrm{G}^{1}$ with $\mathrm{s}_{\mathrm{X}}(x)=\mathrm{r}(g)$;

(2) $\mathrm{m}_{\mathrm{X}} \circ\left(\mathrm{m}_{\mathrm{X}} \circ \mathrm{pr}_{1} ; \mathrm{pr}_{2} \circ \mathrm{pr}_{2}\right)=\mathrm{m}_{\mathrm{X}} \circ\left(\mathrm{pr}_{1} \circ \mathrm{pr}_{1} ; \mathrm{m} \circ \mathrm{pr}_{2}\right), \quad(x \cdot g) \cdot g_{1}=x \cdot\left(g \cdot g_{1}\right)$, for all $x \in \mathrm{X}, g, g_{1} \in \mathrm{G}^{1}$ with $\mathrm{s}_{\mathbf{X}}(x)=\mathrm{r}(g)$ and $\mathrm{s}(g)=\mathrm{r}\left(g_{1}\right)$;

That is, the following diagram commutes:

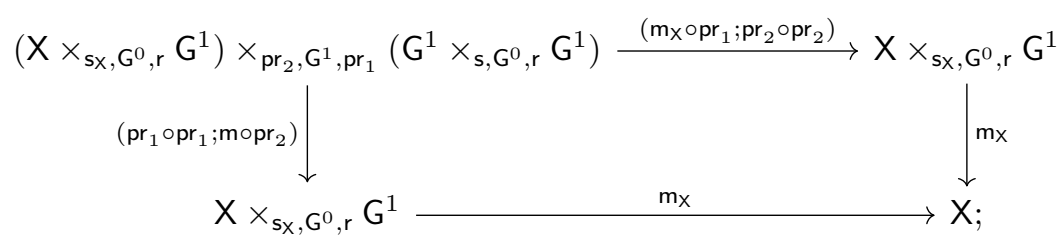

(3) the arrow

$$
\left(\mathrm{m}_{\mathrm{X}} ; \mathrm{pr}_{2}\right): \mathrm{X} \times_{\mathrm{sX}_{\mathrm{X}}, \mathrm{G}^{0}, \mathrm{r}} \mathrm{G}^{1} \longrightarrow \mathrm{X} \times_{\mathrm{sX}_{\mathrm{X}}, \mathrm{G}^{0}, \mathrm{~s}} \mathrm{G}^{1}, \quad(x ; g) \mapsto(x \cdot g ; g),
$$

is an isomorphism.

If the arrow $\mathrm{sX}_{\mathrm{X}} \mathrm{X} \rightarrow \mathrm{G}^{0}$ is a partial cover or cover then this right action is called a partial sheaf or sheaf, respectively.

We call the arrows $\mathrm{s}_{\mathrm{X}}: \mathrm{X} \rightarrow \mathrm{G}^{0}$ and $\mathrm{m}_{\mathrm{X}}: \mathrm{X} \times{ }_{\mathrm{sX}_{\mathrm{X}}, \mathrm{G}^{0}, \mathrm{r}} \mathrm{G}^{1} \rightarrow \mathrm{X}$ the anchor and action maps, respectively.

Remark 5.2. All objects in this definition exist because the range and source maps are covers. The arrows $\left(\mathrm{m}_{\mathrm{x}} \circ \mathrm{pr}_{1} ; \mathrm{pr}_{2} \circ \mathrm{pr}_{2}\right),\left(\mathrm{pr}_{1} \circ \mathrm{pr}_{1} ; \mathrm{m} \circ \mathrm{pr}_{2}\right)$ and $\left(\mathrm{m}_{\mathrm{x}} ; \mathrm{pr}_{2}\right)$ are well-defined because of $(1)$

Definition 5.3. Let $\left(\mathrm{X} ; \mathrm{m}_{\mathrm{X}} ; \mathrm{s}_{\mathrm{X}}\right)$ be a right $\mathrm{G}$-action. An arrow $f: \mathrm{X} \rightarrow \mathrm{Y}$ is called $\mathrm{G}$-invariant if $f \circ \mathrm{m}_{\mathbf{X}}=f \circ \mathrm{pr}_{1}$. Elementwise, $f(x \cdot g)=f(x)$.

Lemma 5.4. Let $\mathrm{G}=\left(\mathrm{G}^{0}, \mathrm{G}^{1}, \mathrm{r}, \mathrm{s}, \mathrm{m}\right)$ be a groupoid and let $\left(\mathrm{X} ; \mathrm{m}_{\mathrm{X}} ; \mathrm{s} \mathrm{X}\right)$ be a right G-action. Let $x_{1}, x_{2}$ and $g$ be such that $\left(x_{1} ; g\right),\left(x_{2} ; g\right) \in \mathcal{C}\left(-, \mathrm{X} \times_{\mathbf{s}_{\mathrm{X}}, \mathrm{G}^{0}, \mathrm{r}} \mathrm{G}^{1}\right)$ are well-defined arrows and $\mathrm{m}_{\mathbf{X}} \circ\left(x_{1} ; g\right)=\mathrm{m}_{\mathbf{X}} \circ\left(x_{2} ; g\right)$, then $x_{1}=x_{2}$.

Proof. We have

$$
\begin{aligned}
\left(\mathrm{m}_{\mathbf{X}} ; \mathrm{pr}_{2}\right) \circ\left(x_{1} ; g\right) & =\left(\mathrm{m}_{\mathbf{X}} \circ\left(x_{1} ; g\right) ; g\right) \\
& =\left(\mathrm{m}_{\mathbf{X}} \circ\left(x_{2} ; g\right) ; g\right) \\
& =\left(\mathrm{m}_{\mathbf{X}} ; \mathrm{pr}_{2}\right) \circ\left(x_{2} ; g\right) .
\end{aligned}
$$

We know that the arrow $\left(\mathrm{m}_{\mathbf{x}} ; \mathrm{pr}_{2}\right)$ is an isomorphism. Therefore, $\left(x_{1} ; g\right)=\left(x_{2} ; g\right)$, hence $x_{1}=x_{2}$.

Remark 5.5. The elementwise notation of Lemma 5.4 is the following. If $x_{1} \cdot g=x_{2} \cdot g$ then $x_{1}=x_{2}$.

Lemma 5.6. Let $\mathrm{G}$ be a groupoid and let $\left(\mathrm{X} ; \mathrm{m}_{\mathrm{X}} ; \mathrm{s}_{\mathrm{X}}\right)$ be a right $\mathrm{G}$-action. Then the action map $\mathrm{m}_{\mathrm{X}}: \mathrm{X} \times{ }_{\mathrm{sX}_{\mathrm{X}}, \mathrm{G}^{0}, \mathrm{r}} \mathrm{G}^{1} \rightarrow \mathrm{X}$ is a cover. Moreover it splits by the arrow $\left(\mathrm{id}_{\mathrm{X}} ; \mathrm{u} \circ \mathrm{s}_{\mathrm{X}}\right): \mathrm{X} \rightarrow \mathrm{X} \times_{\mathrm{s}_{\mathrm{X}}, \mathrm{G}^{0}, \mathrm{r}} \mathrm{G}^{1}$. 
Proof. The action map $\mathrm{m}_{\mathrm{X}}: \mathrm{X} \times_{\mathrm{sX}_{\mathrm{X}}, \mathrm{G}^{0}, \mathrm{r}} \mathrm{G}^{1} \rightarrow \mathrm{X}$ is the following composition:

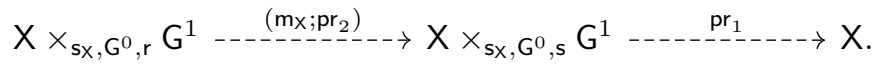

Also, the coordinate projection $\mathrm{pr}_{1}: \mathrm{X} \times_{\mathrm{sX}_{\mathrm{X}}, \mathrm{G}^{0}, \mathrm{~s}} \mathrm{G}^{1} \rightarrow \mathrm{X}$ is a partial cover because it is a pull-back of the source map $s: G^{1} \rightarrow G^{0}$, and the arrow $\left(m_{x} ; p_{2}\right)$ is a partial cover because it is an isomorphism. Therefore, their composition $\mathrm{m}_{\mathrm{X}}$ is a partial cover, too.

Consider the following arrow

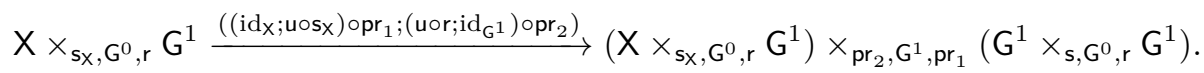

This map is defined elementwise by $(x ; g) \mapsto\left(\left(x ; 1_{\mathrm{sx}_{\mathrm{X}}(x)}\right) ;\left(1_{\mathrm{r}(g)} ; g\right)\right)$. It is well-defined because $\mathrm{r}\left(1_{\mathrm{s}_{\mathrm{X}}(x)}\right)=\mathrm{sX}(x), \mathrm{s}\left(1_{\mathrm{r}(g)}\right)=\mathrm{r}(g)$ and $1_{\mathrm{s}_{\mathrm{X}}(x)}=1_{\mathrm{r}(g)}$, for all $x \in \mathrm{X}, g \in \mathrm{G}^{1}$ with $\mathrm{s}_{\mathrm{X}}(x)=\mathrm{r}(g)$. The condition $(2)$ in Definition 5.1 implies

$$
\begin{aligned}
\left(x \cdot 1_{\mathrm{sX}(x)}\right) \cdot g & =x \cdot\left(1_{\mathrm{r}(g)} \cdot g\right) \\
& =x \cdot g .
\end{aligned}
$$

Because of Lemma 5.4 we can deduce that $x \cdot 1_{\mathrm{sX}(x)}=x$, for all $x \in \mathrm{X}$. Therefore, $\mathrm{m}_{\mathrm{X}} \circ\left(\mathrm{id} \mathrm{X} ; \mathrm{u} \circ \mathrm{s}_{\mathrm{X}}\right) \circ \mathrm{pr}_{1}=\mathrm{pr}_{1}$. Also we know that $\mathrm{pr}_{1}: \mathrm{X} \times_{\mathrm{sX}_{\mathrm{X}}, \mathrm{G}^{0}, \mathrm{r}} \mathrm{G}^{1} \rightarrow \mathrm{X}$ is a cover because it is a pull-back of the range map $r: G^{1} \rightarrow G^{0}$, which is a cover and splits by the unit map $\mathrm{u}: \mathrm{G}^{0} \rightarrow \mathrm{G}^{1}$. Therefore, Corollary 2.7 works. So $\mathrm{pr}_{1}$ is a coequaliser. Hence it is epic and we can infer that

$$
\mathrm{m}_{\mathrm{X}} \circ\left(\mathrm{id} \mathrm{x} ; \mathrm{u} \circ \mathrm{s}_{\mathrm{X}}\right)=\mathrm{id}_{\mathrm{X}} \text {. }
$$

So the action map $m_{\mathbf{X}}$ is a partial cover and it splits by (idx; $\left.u \circ s_{\mathbf{X}}\right)$. Therefore, it is a cover by Lemma 2.5 .

Lemma 5.7. Let $\mathrm{G}=\left(\mathrm{G}^{0}, \mathrm{G}^{1}, \mathrm{r}, \mathrm{s}, \mathrm{m}, \mathrm{u}, \mathrm{i}\right)$ be a groupoid. Assume that the triple $\left(\mathrm{X} ; \mathrm{m}_{\mathrm{X}} ; \mathrm{s}_{\mathrm{X}}\right)$ satisfies the conditions $(1),(2)$ and that the equation $(5.2)$ holds. Then $\left(\mathrm{X} ; \mathrm{m}_{\mathrm{X}} ; \mathrm{s}_{\mathrm{X}}\right)$ is a right $\mathrm{G}-$ action.

Proof. We only need to prove that the arrow (5.1) is an isomorphism. Consider the arrow

$$
\mathrm{X} \times_{\mathrm{sX}_{\mathrm{X}}, \mathrm{G}^{0}, \mathrm{~s}} \mathrm{G}^{1} \stackrel{\left(\mathrm{m}_{\mathrm{X}} \circ\left(\mathrm{pr}_{1} ; \mathrm{iopr}_{2}\right) ; \mathrm{pr}_{2}\right)}{\longrightarrow} \mathrm{X} \times_{\mathrm{sX}_{\mathrm{X}}, \mathrm{G}^{0}, \mathrm{r}} \mathrm{G}^{1}, \quad(x ; g) \mapsto\left(x \cdot g^{-1} ; g\right) .
$$

It is well-defined because $\mathrm{sx}_{\mathbf{X}}\left(x \cdot g^{-1}\right)=\mathrm{s}\left(g^{-1}\right)=\mathrm{r}(g)$. Consider the composition

$$
\begin{aligned}
\left(\mathrm{m}_{\mathbf{X}} ; \mathrm{pr}_{2}\right)\left(\left(\mathrm{m}_{\mathbf{X}} \circ\left(\mathrm{pr}_{1} ; \mathrm{i} \circ \mathrm{pr}_{2}\right) ; \mathrm{pr}_{2}\right)(x ; g)\right) & =\left(\mathrm{m}_{\mathbf{X}} ; \mathrm{pr}_{2}\right)\left(x \cdot g^{-1} ; g\right) \\
& =\left(\left(x \cdot g^{-1}\right) \cdot g ; g\right) \\
& =\left(x \cdot\left(g^{-1} \cdot g\right) ; g\right) \\
& =\left(x \cdot 1_{\mathrm{s}(g)} ; g\right) \\
& =\left(x \cdot 1_{\mathrm{s}_{X}(x)} ; g\right) \\
& =(x ; g)
\end{aligned}
$$

for all $x \in \mathrm{X}, g \in \mathrm{G}^{1}$ with $\mathrm{s}_{\mathbf{X}}(x)=\mathrm{s}(g)$. We also have

$$
\begin{aligned}
\left(\mathrm{m}_{\mathrm{X}} \circ\left(\mathrm{pr}_{1} ; \mathrm{i} \circ \mathrm{pr}_{2}\right) ; \mathrm{pr}_{2}\right)\left(\left(\mathrm{m}_{\mathrm{X}} ; \mathrm{pr}_{2}\right)(x ; g)\right) & =\left(\mathrm{m}_{\mathrm{X}} \circ\left(\mathrm{pr}_{1} ; \mathrm{i} \circ \mathrm{pr}_{2}\right) ; \mathrm{pr}_{2}\right)(x \cdot g ; g) \\
& =\left((x \cdot g) \cdot g^{-1} ; g\right) \\
& =\left(x \cdot\left(g \cdot g^{-1}\right) ; g\right) \\
& =\left(x \cdot 1_{\mathrm{r}(g)} ; g\right) \\
& =\left(x \cdot 1_{\mathrm{sX}(x)} ; g\right) \\
& =(x ; g)
\end{aligned}
$$


for all $x \in \mathrm{X}, g \in \mathrm{G}^{1}$ with $\mathrm{sx}(x)=\mathrm{r}(g)$. Therefore, the arrow $\left(\mathrm{m}_{\mathrm{X}} \circ\left(\mathrm{pr}_{1} ; \mathrm{i} \circ \mathrm{pr}_{2}\right) ; \mathrm{pr}_{2}\right)$ is an inverse of $\left(\mathrm{m}_{\mathbf{X}} ; \mathrm{pr}_{2}\right)$. Thus $[5.2)$ is an isomorphism. Hence $\left(\mathrm{X} ; \mathrm{m}_{\mathrm{X}} ; \mathrm{sX}_{\mathrm{X}}\right)$ is a right G-action.

The definitions of right and left actions are similar.

Definition 5.8. Let $\mathrm{G}=\left(\mathrm{G}^{0}, \mathrm{G}^{1}, \mathrm{r}, \mathrm{s}, \mathrm{m}, \mathrm{u}, \mathrm{i}\right)$ be a groupoid. A left $\mathrm{G}$-action $\left(X ; m_{X} ; r_{X}\right)$ is an object $X$ with arrows $r_{X}: X \rightarrow G^{0}$ and $m_{X}: G^{1} \times_{s, G^{0}, r_{X}} X \rightarrow X$ such that

(1) $\mathrm{r}_{\mathrm{X}} \circ \mathrm{m}_{\mathrm{X}}=\mathrm{r} \circ \mathrm{pr}_{1}, \quad \mathrm{rX}_{\mathbf{X}}(g \cdot x)=\mathrm{r}(g)$ for all $x \in \mathrm{X}, g \in \mathrm{G}^{1}$ with $\mathrm{s}(g)=\mathrm{rX}_{\mathbf{X}}(x)$;

(2) $\mathrm{m}_{\mathrm{X}} \circ\left(\mathrm{pr}_{1} \circ \mathrm{pr}_{1} ; \mathrm{m}_{\mathrm{X}} \circ \mathrm{pr}_{2}\right)=\mathrm{m}_{\mathrm{X}} \circ\left(\mathrm{m} \circ \mathrm{pr}_{1} ; \mathrm{pr}_{2} \circ \mathrm{pr}_{2}\right), \quad g \cdot\left(g_{1} \cdot x\right)=\left(g \cdot g_{1}\right) \cdot x$, for all $x \in \mathrm{X}, g, g_{1} \in \mathrm{G}^{1}$ with $\mathrm{s}(g)=\mathrm{r}\left(g_{1}\right)$ and $\mathrm{s}\left(g_{1}\right)=\mathrm{r}_{\mathbf{X}}(x)$;

That is, the following diagram commutes:

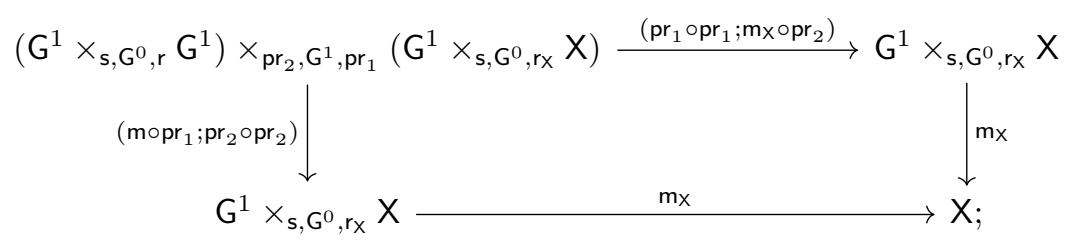

(3) the arrow

$$
\left(\mathrm{pr}_{1} ; \mathrm{m}_{\mathrm{X}}\right): \mathrm{G}^{1} \times_{\mathrm{s}, \mathrm{G}^{0}, \mathrm{r}_{\mathrm{X}}} \mathrm{X} \longrightarrow \mathrm{G}^{1} \times_{\mathrm{r}, \mathrm{G}^{0}, \mathrm{r}_{\mathrm{X}}} \mathrm{X}, \quad(g ; x) \mapsto(g ; g \cdot x)
$$

is an isomorphism.

If the arrow $r_{X}: X \rightarrow G^{0}$ is a partial cover or cover then this left action is called a partial sheaf or sheaf, respectively.

Remark 5.9. Lemma 5.4 has an analogue for left actions. If we have the elements $x_{1}, x_{2}$ and $g$ with all required properties and $g \cdot x_{1}=g \cdot x_{2}$, then $x_{1}=x_{2}$. The proof is similar. We have to use the isomorphism (5.3) instead of (5.1). The elementwise notation of this fact is the following. If $g \cdot x_{1}=g \cdot x_{2}$ then $x_{1}=x_{2}$.

Remark 5.10. The action map $\mathrm{m}_{\mathrm{X}}$ of a left $\mathrm{G}$-action is a splitting cover. The proof is the same as the proof of Lemma 5.6. We just have to use the isomorphism (5.3)

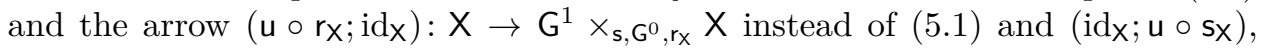
respectively.

Remark 5.11. Lemma 5.7 has an analogue for left actions. If the triple $\left(\mathrm{X} ; \mathrm{m}_{\mathbf{X}} ; \mathrm{r}_{\mathbf{X}}\right)$ satisfies the conditions (1) (2) in Definition 5.8 and the equation $1_{\mathrm{rX}_{\mathrm{X}}(x)} \cdot x=x$ holds, then $\left(X ; m_{X} ; s_{X}\right)$ is a left G-action. The proof is almost the same. We just need to use the arrow $\left(\mathrm{pr}_{1} ; \mathrm{m}_{\mathrm{X}} \circ\left(\mathrm{i} \circ \mathrm{pr}_{1} ; \mathrm{pr}_{2}\right)\right)$ instead of $\left(\mathrm{m}_{\mathrm{X}} \circ\left(\mathrm{pr}_{1} ; \mathrm{i} \circ \mathrm{pr}_{2}\right) ; \mathrm{pr}_{2}\right)$.

Definition 5.12. Let $G=\left(G^{0}, G^{1}, r, s, m, u, i\right)$ be a groupoid and let $X$ and $Y$ be right $\mathrm{G}$-actions. An arrow $f: \mathrm{X} \rightarrow \mathrm{Y}$ is called a right $\mathrm{G}$-map if it satisfies the following conditions:

(1) $\mathrm{s}_{\mathrm{X}}=\mathrm{s}_{\mathrm{Y}} \circ f, \quad \mathrm{~s}_{\mathrm{X}}(x)=\mathrm{s}_{\mathrm{Y}}(f(x)), \quad \forall x \in \mathrm{X}$;

(2) $f \circ \mathrm{m}_{\mathrm{X}}=\mathrm{m}_{\mathrm{Y}} \circ\left(f \circ \mathrm{pr}_{1} ; \mathrm{pr}_{2}\right), \quad f(x \cdot g)=f(x) \cdot g$ for all $x \in \mathrm{X}, g \in \mathrm{G}^{1}$ with $\mathrm{s}_{\mathbf{X}}(x)=\mathrm{r}(g)$; that is, the following diagram commutes:

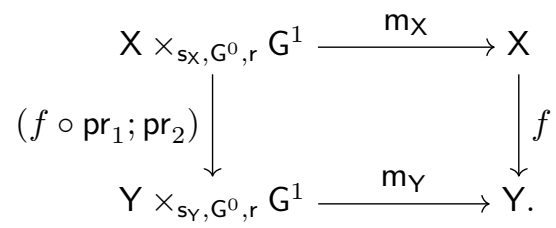

The arrow $\left(f \circ \mathrm{pr}_{1} ; \mathrm{pr}_{2}\right): \mathrm{X} \times_{\mathrm{sX}_{\mathrm{X}}, \mathrm{G}^{0}, \mathrm{r}} \mathrm{G}^{1} \rightarrow \mathrm{Y} \times_{\mathrm{sY}_{\mathrm{Y}} \mathrm{G}^{0}, \mathrm{r}} \mathrm{G}^{1}$ is well-defined because $\mathrm{s}_{\mathbf{Y}}(f(x))=\mathrm{s}_{\mathbf{X}}(x)=\mathrm{r}(g)$ for all $x \in \mathrm{X}, g \in \mathrm{G}^{1}$ with $\mathrm{s}_{\mathbf{X}}(x)=\mathrm{r}(g)$. 
Remark 5.13. If $f: \mathrm{X} \rightarrow \mathrm{Y}$ is a right $\mathrm{G}$-map and it is invertible, then its inverse is a right G-map, too. We just need to remark that $\left(f^{-1} \circ \mathrm{pr}_{1} ; \mathrm{pr}_{2}\right)$ is inverse to $\left(f \circ \mathrm{pr}_{1} ; \mathrm{pr}_{2}\right)$, and the needed commutation works automatically.

Lemma 5.14. For any groupoid $\mathrm{G}=\left(\mathrm{G}^{0}, \mathrm{G}^{1}, \mathrm{r}, \mathrm{s}, \mathrm{m}, \mathrm{u}, \mathrm{i}\right)$, the right $\mathrm{G}$-actions as objects and the right $\mathrm{G}-m a p s$ as arrows form a category. Denote it by $\mathcal{C}(\mathrm{G})$.

Proof. It is enough to check that the composition of two composable right G-maps is a right $\mathrm{G}$-map too. Let $f_{1}: \mathrm{X} \rightarrow \mathrm{Y}$ and $f_{2}: \mathrm{Y} \rightarrow \mathrm{Z}$ be right $\mathrm{G}$-maps. We have $\mathrm{s}_{\mathrm{Z}}\left(f_{2}\left(f_{1}(x)\right)\right)=\mathrm{sY}\left(f_{1}(x)\right)=\mathrm{sX}_{\mathbf{X}}(x)$ and $f_{2}\left(f_{1}(x \cdot g)\right)=f_{2}\left(f_{1}(x) \cdot g\right)=f_{2}\left(f_{1}(x)\right) \cdot g$ for all $x \in \mathrm{X}, g \in \mathrm{G}^{1}$ with $\mathrm{s} \times(x)=\mathrm{r}(g)$. So the composition $f_{2} \circ f_{1}$ satisfies the conditions. Hence it is a G-map too.

Remark 5.15. There are full subcategories of right partial G-sheaves and right G-sheaves. Denote them by $\mathcal{C}_{\mathcal{F}_{p}}(\mathrm{G})$ and $\mathcal{C}_{\mathcal{F}}(\mathrm{G})$, respectively.

Analogously, we can define the categories of left G-actions, left partial G-sheaves and left G-sheaves.

Definition 5.16. Let $G=\left(G^{0}, G^{1}, r, s, m, u, i\right)$ be a groupoid and let $X$ and $Y$ be left $\mathrm{G}$-actions. The arrow $f: \mathrm{X} \rightarrow \mathrm{Y}$ is called a left $\mathrm{G}$-map if it satisfies the following conditions:

(1) $\mathrm{r}_{\mathrm{X}}=\mathrm{r}_{\mathbf{Y}} \circ f, \quad \mathrm{r}_{\mathrm{X}}(x)=\mathrm{r}_{\mathbf{Y}}(f(x))$ for all $x \in \mathrm{X}$;

(2) $f \circ \mathrm{m}_{\mathrm{X}}=\mathrm{m}_{\mathrm{Y}} \circ\left(\mathrm{pr}_{1} ; f \circ \mathrm{pr}_{2}\right), \quad f(g \cdot x)=g \cdot f(x)$ for all $x \in \mathrm{X}, g \in \mathrm{G}^{1}$ with $\mathrm{s}(g)=\mathrm{r}_{\mathbf{X}}(x)$; that is, the following diagram commutes:

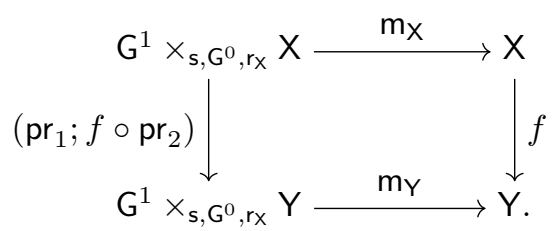

The arrow $\left(\mathrm{pr}_{1} ; f \circ \mathrm{pr}_{2}\right): \mathrm{G}^{1} \times_{\mathrm{sX}_{\mathrm{X}}, \mathrm{G}^{0}, \mathrm{r}} \mathrm{X} \rightarrow \mathrm{G}^{1} \times_{\mathrm{sY}_{\mathrm{Y}}, \mathrm{G}^{0}, \mathrm{r}} \mathrm{Y}$ is well-defined because $\mathrm{r}_{\mathbf{Y}}(f(x))=\mathrm{rX}_{\mathbf{X}}(x)=\mathrm{s}(g)$.

Remark 5.17. The left G-actions as objects and the left G-maps as arrows form a category. The proof is similar to the proof of the Lemma 5.14

Lemma 5.18. The categories of right and left $\mathrm{G}$-actions are isomorphic.

Proof. We turn a right $\mathrm{G}$-action $\left(\mathrm{X} ; \mathrm{m}_{\mathbf{X}} ; \mathrm{s}_{\mathrm{X}}\right)$ into a left $\mathrm{G}$-action $\left(\mathrm{X} ; \hat{\mathrm{m}}_{\mathrm{X}} ; \mathrm{r}_{\mathbf{X}}\right)$ by $\mathrm{r}_{\mathbf{X}}=\mathrm{s}_{\mathrm{X}}$ and $\hat{\mathrm{m}}_{\mathrm{X}}=\mathrm{m}_{\mathrm{X}} \circ\left(\mathrm{pr}_{2} ; \mathrm{i} \circ \mathrm{pr}_{1}\right)$. Elementwise, $g \cdot x=x \cdot g^{-1}$ for all $x \in \mathrm{X}, g \in \mathrm{G}^{1}$ with $\mathrm{s}(g)=\mathrm{r}_{\mathbf{X}}(x)$. The arrow

$$
\mathrm{G}^{1} \times_{\mathrm{s}, \mathrm{G}^{0}, \mathrm{rx}_{\mathrm{X}}} \mathrm{X} \stackrel{\left(\mathrm{pr}_{2} ; \mathrm{iopr}_{1}\right)}{\longrightarrow} \mathrm{X} \times \times_{\mathrm{s}_{\mathrm{X}}, \mathrm{G}^{0}, \mathrm{r}} \mathrm{G}^{1}
$$

is well-defined because $r\left(g^{-1}\right)=s(g)=r_{X}(x)=s_{X}(x)$.

We have to prove that $\left(\mathrm{X} ; \mathrm{m}_{\mathbf{X}} \circ\left(\mathrm{pr}_{2} ; \mathrm{i} \circ \mathrm{pr}_{1}\right) ; \mathrm{sX}_{\mathrm{X}}\right)$ defines a left G-action. It is clear that $\mathrm{s}_{\mathbf{X}}(g \cdot x)=\mathrm{s}_{\mathbf{X}}\left(x \cdot g^{-1}\right)=\mathrm{s}\left(g^{-1}\right)=\mathrm{r}(g)$ for all $x \in \mathrm{X}, g \in \mathrm{G}^{1}$ with $\mathrm{s}(g)=\mathrm{rX}(x)$. So we have the property (1) in Definition 5.8

We also have

$$
\begin{aligned}
g \cdot\left(g_{1} \cdot x\right) & =g \cdot\left(x \cdot g_{1}^{-1}\right) \\
& =\left(x \cdot g_{1}^{-1}\right) \cdot g^{-1} \\
& =x \cdot\left(g_{1}^{-1} \cdot g^{-1}\right) \\
& =x \cdot\left(g \cdot g_{1}\right)^{-1} \\
& =\left(g \cdot g_{1}\right) \cdot x
\end{aligned}
$$


for all $x \in \mathrm{X}, g, g_{1} \in \mathrm{G}^{1}$ with $\mathrm{s}(g)=\mathrm{r}\left(g_{1}\right)$ and $\mathrm{s}\left(g_{1}\right)=\mathrm{r}_{\mathbf{X}}(x)$. So the property $(2)$ is satisfied.

The arrow

$$
\mathrm{X} \times_{\mathrm{sX}, \mathrm{G}^{0}, \mathrm{r}} \mathrm{G}^{1} \stackrel{\left(\mathrm{iopr}_{2} ; \mathrm{pr}_{1}\right)}{\longrightarrow} \mathrm{G}^{1} \times_{\mathrm{s}, \mathrm{G}^{0}, \mathrm{rX}} \mathrm{X}, \quad(x ; g) \mapsto\left(g^{-1} ; x\right)
$$

is well-defined because $\mathrm{s}\left(g^{-1}\right)=\mathrm{r}(g)=\mathrm{s}_{\mathbf{X}}(x)=\mathrm{rX}_{\mathbf{X}}(x)$ for all $x \in \mathrm{X}, g \in \mathrm{G}^{1}$ with $\mathrm{s}(g)=\mathrm{r}_{\mathbf{X}}(x)$. We have

$$
\begin{aligned}
\left(\mathrm{i} \circ \mathrm{pr}_{2} ; \mathrm{pr}_{1}\right)\left(\left(\mathrm{pr}_{2} ; \mathrm{i} \circ \mathrm{pr}_{1}\right)(g ; x)\right) & =\left(\mathrm{i} \circ \mathrm{pr}_{2} ; \mathrm{pr}_{1}\right)\left(x ; g^{-1}\right) \\
& =\left(\left(g^{-1}\right)^{-1} ; x\right) \\
& =(g ; x)
\end{aligned}
$$

for all $x \in \mathrm{X}, g \in \mathrm{G}^{1}$ with $\mathrm{s}(g)=\operatorname{r\times }(x)$ and

$$
\begin{aligned}
\left(\mathrm{pr}_{2} ; \mathrm{i} \circ \mathrm{pr}_{1}\right)\left(\left(\mathrm{i} \circ \mathrm{pr}_{2} ; \mathrm{pr}_{1}\right)(x ; g)\right) & =\left(g^{-1} ; x\right) \\
& \left.=\mathrm{pr}_{2} ; \mathrm{i} \circ \mathrm{pr}_{1}\right)\left(x ;\left(g^{-1}\right)^{-1}\right) \\
& =(x ; g)
\end{aligned}
$$

for all $x \in \mathrm{X}, g \in \mathrm{G}^{1}$ with $\mathrm{sX}(x)=\mathrm{r}(g)$. Therefore, $\left(\mathrm{pr}_{2} ; \mathrm{i} \circ \mathrm{pr}_{1}\right)$ is an isomorphism. Analogously, we have the following isomorphism

$$
\left(\mathrm{i} \circ \mathrm{pr}_{2} ; \mathrm{pr}_{1}\right): \mathrm{X} \times_{\mathrm{sX}_{\mathrm{X}}, \mathrm{G}^{0}, \mathrm{~s}} \mathrm{G}^{1} \stackrel{\sim}{\rightarrow} \mathrm{G}^{1} \times_{\mathrm{r}, \mathrm{G}^{0}, \mathrm{r}_{\mathrm{X}}} \mathrm{X}, \quad(x ; g) \mapsto\left(g^{-1} ; x\right) .
$$

Consider the composition of isomorphisms

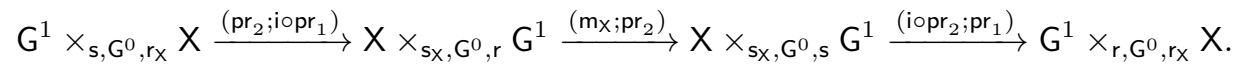

We have

$$
\begin{aligned}
\left(\mathrm{i} \circ \mathrm{pr}_{2} ; \mathrm{pr}_{1}\right)\left(\left(\mathrm{m}_{\mathrm{X}} ; \mathrm{pr}_{2}\right)\left(\left(\mathrm{pr}_{2} ; \mathrm{i} \circ \mathrm{pr}_{1}\right)(g ; x)\right)\right) & =\left(\mathrm{i} \circ \mathrm{pr}_{2} ; \mathrm{pr}_{1}\right)\left(\left(\mathrm{m}_{\mathbf{X}} ; \mathrm{pr}_{2}\right)\left(x ; g^{-1}\right)\right) \\
& =\left(\mathrm{i} \circ \mathrm{pr}_{2} ; \mathrm{pr}_{1}\right)\left(x \cdot g^{-1} ; g^{-1}\right) \\
& =\left(g^{-1} ; x \cdot g^{-1}\right) \\
& =(g ; g \cdot x) \\
& =\left(\mathrm{pr}_{1} ; \hat{\mathrm{m}}_{\mathrm{X}}\right)(g ; x)
\end{aligned}
$$

for all $x \in \mathrm{X}, g \in \mathrm{G}^{1}$ with $\mathrm{s}(g)=\mathrm{r}_{\mathbf{X}}(x)$. Hence the arrow $\left(\mathrm{pr}_{1} ; \hat{\mathrm{m}}_{\mathbf{X}}\right)$ is an isomorphism. So the property $(3)$ is satisfied, too, and $\left(\mathrm{X} ; \mathrm{m}_{\mathrm{X}} \circ\left(\mathrm{pr}_{2} ; \mathrm{i} \circ \mathrm{pr}_{1}\right) ; \mathrm{sX}\right)$ is a left G-action.

Analogously, we can prove that if $\left(X ; m_{X} ; r_{X}\right)$ is a left $G$-action then there is a corresponding right $\mathrm{G}$-action $\left(\mathrm{X} ; \mathrm{m}_{\mathrm{X}} \circ\left(\mathrm{i} \circ \mathrm{pr}_{2} ; \mathrm{pr}_{1}\right) ; \mathrm{r}_{\mathrm{X}}\right)$. Finally, we can deduce that this construction of the corresponding left and right $\mathrm{G}$-actions gives an isomorphism between these categories because the arrows $\left(\mathrm{i} \circ \mathrm{pr}_{2} ; \mathrm{pr}_{1}\right)$ and $\left(\mathrm{pr}_{2} ; \mathrm{i} \circ \mathrm{pr}_{1}\right)$ are inverses of each other.

Remark 5.19. In the proof of Lemma 5.18 during the construction of the corresponding left and right G-actions we do not change the anchor map. Therefore, the categories of left and right partial G-sheaves are isomorphic and the categories of left and right G-sheaves are isomorphic, too.

Lemma 5.20. Let $\mathrm{G}=\left(\mathrm{G}^{0}, \mathrm{G}^{1}, \mathrm{r}, \mathrm{s}, \mathrm{m}, \mathrm{u}, \mathrm{i}\right)$ be a groupoid. Suppose that $\left(\mathrm{X} ; \mathrm{m}_{\mathrm{X}} ; \mathrm{s} \mathrm{X}\right)$, $\left(\mathrm{X}_{1} ; \mathrm{m}_{\mathrm{X}_{1}} ; \mathrm{s}_{\mathrm{X}_{1}}\right)$ and $\left(\mathrm{X}_{2} ; \mathrm{m}_{\mathrm{X}_{2}} ; \mathrm{s}_{\mathrm{X}_{2}}\right)$ are the right $\mathrm{G}$-actions and that $f_{1}: \mathrm{X}_{1} \rightarrow \mathrm{X}$ and $f_{2}: \mathrm{X}_{2} \rightarrow \mathrm{X}$ are the right $\mathrm{G}$-maps. If the fibre product $\mathrm{X}_{1} \times_{f_{1}, \mathbf{X}_{f_{2}}} \mathrm{X}_{2}$ exists in $\mathcal{C}$ then it exists in $\mathcal{C}(\mathrm{G})$, too. Call it the fibre product of $\mathrm{G}-$ maps with the same target.

Proof. We must define a right G-action $\left(\mathrm{X}_{1} \times_{f_{1}, \mathrm{X}, f_{2}} \mathrm{X}_{2} ; \mathrm{m}_{0} ; \mathrm{s}_{0}\right)$. Let the anchor map be $\mathrm{s}_{0}\left(x_{1} ; x_{2}\right)=\mathrm{s}_{X}\left(f_{1}\left(x_{1}\right)\right)$ and let $\left(x_{1} ; x_{2}\right) \cdot g=\left(x_{1} \cdot g ; x_{2} \cdot g\right)$ for all $x_{1} \in \mathrm{X}_{1}$, $x_{2} \in \mathrm{X}_{2}$ and $g \in \mathrm{G}^{1}$ with $f_{1}\left(x_{1}\right)=f_{2}\left(x_{2}\right)$ and $\mathrm{s}_{0}\left(x_{1} ; x_{2}\right)=\mathrm{r}(g)$. This action map is well-defined because $f_{1}\left(x_{1} \cdot g\right)=f_{1}\left(x_{1}\right) \cdot g=f_{2}\left(x_{2}\right) \cdot g=f_{2}\left(x_{2} \cdot g\right)$. Also we have 
$\mathrm{s}_{0}\left(\left(x_{1} ; x_{2}\right) \cdot g\right)=\mathrm{s}_{0}\left(x_{1} \cdot g ; x_{2} \cdot g\right)=\mathrm{s}_{\mathbf{X}}\left(f_{1}\left(x_{1} \cdot g\right)\right)=\mathrm{s}_{\mathbf{X}}\left(f_{1}\left(x_{1}\right) \cdot g\right)=\mathrm{r}(g)$. Hence the property (1) holds. Associativity holds because

$$
\begin{aligned}
\left(x_{1} ; x_{2}\right) \cdot\left(g_{1} \cdot g_{2}\right) & =\left(x_{1} \cdot\left(g_{1} \cdot g_{2}\right) ; x_{2} \cdot\left(g_{1} \cdot g_{2}\right)\right) \\
& =\left(\left(x_{1} \cdot g_{1}\right) \cdot g_{2} ;\left(x_{2} \cdot g_{1}\right) \cdot g_{2}\right) \\
& =\left(x_{1} \cdot g_{1} ; x_{2} \cdot g_{1}\right) \cdot g_{2} \\
& =\left(\left(x_{1} ; x_{2}\right) \cdot g_{1}\right) \cdot g_{2}
\end{aligned}
$$

for all $x_{1} \in \mathrm{X}_{1}, x_{2} \in \mathrm{X}_{2}$ and $g_{1}, g_{2} \in \mathrm{G}^{1}$ with $f_{1}\left(x_{1}\right)=f_{2}\left(x_{2}\right), \mathrm{s}_{0}\left(x_{1} ; x_{2}\right)=\mathrm{r}\left(g_{1}\right)$ and $\mathrm{s}\left(g_{1}\right)=\mathrm{r}\left(g_{2}\right)$. Also, we have $\left(x_{1} ; x_{2}\right) \cdot 1_{\mathrm{sx}_{\mathrm{X}}\left(f_{1}\left(x_{1}\right)\right)}=\left(x_{1} \cdot 1_{\mathrm{sx}_{1}\left(x_{1}\right)} ; x_{2} \cdot 1_{\mathrm{sx}_{2}\left(x_{2}\right)}\right)=\left(x_{1} ; x_{2}\right)$. Therefore, $\left(\mathrm{X}_{1} \times_{f_{1}, \mathrm{X}, f_{2}} \mathrm{X}_{2} ; \mathrm{m}_{0} ; \mathrm{s}_{0}\right)$ is a right $\mathrm{G}$-action by Lemma 5.7

We must also show that the coordinate projections $\mathrm{pr}_{1}: \mathrm{X}_{1} \times_{f_{1}, \mathrm{X}, f_{2}} \mathrm{X}_{2} \rightarrow \mathrm{X}_{1}$ and $\mathrm{pr}_{2}: \mathrm{X}_{1} \times_{f_{1}, \mathrm{X}, f_{2}} \mathrm{X}_{2} \rightarrow \mathrm{X}_{2}$ are G-maps. We have

$$
\begin{aligned}
\operatorname{pr}_{1}\left(\left(x_{1} ; x_{2}\right) \cdot g\right) & =\operatorname{pr}_{1}\left(x_{1} \cdot g ; x_{2} \cdot g\right) \\
& =x_{1} \cdot g \\
& =\operatorname{pr}_{1}\left(x_{1} ; x_{2}\right) \cdot g .
\end{aligned}
$$

Analogously, the second coordinate projection $\mathrm{pr}_{2}$ is a G-map.

Now consider a G-action (A; $\hat{\mathrm{m}} ; \hat{\mathrm{s}})$ and two G-maps $\alpha: \mathrm{A} \rightarrow \mathrm{X}_{1}$ and $\beta: \mathrm{A} \rightarrow \mathrm{X}_{2}$ such that $f_{1} \circ \alpha=f_{2} \circ \beta$. The unique arrow $(\alpha ; \beta): \mathrm{A} \rightarrow \mathrm{X}_{1} \times_{f_{1}, \mathrm{X}, f_{2}} \mathrm{X}_{2}$ is a $\mathrm{G}$-map because

$$
\begin{aligned}
(\alpha ; \beta)(a \cdot g) & =(\alpha(a \cdot g) ; \beta(a \cdot g)) \\
& =(\alpha(a) \cdot g ; \beta(a) \cdot g) \\
& =(\alpha(a) ; \beta(a)) \cdot g \\
& =(\alpha ; \beta)(a) \cdot g
\end{aligned}
$$

for all $a \in \mathrm{A}$ and $g \in \mathrm{G}^{1}$ with $\hat{\mathrm{s}}(a)=\mathrm{r}(g)$. Therefore, $\mathrm{X}_{1} \times_{f_{1}, \mathrm{X}, f_{2}} \mathrm{X}_{2}$ is a fibre product in $\mathcal{C}(\mathrm{G})$, too.

\subsection{Examples of groupoid actions.}

Example 5.21. If $\mathrm{G}$ is a groupoid then there is a right $\mathrm{G}$-sheaf $\left(\mathrm{G}^{0} ; \mathrm{s} \circ \mathrm{pr}_{2} ; \mathrm{id}_{\mathrm{G}^{0}}\right)$. It is clear that the property (1) in Definition 5.1 is satisfied. The property (3) is satisfied because the arrow $\left(\mathrm{s} \circ \mathrm{pr}_{2} ; \mathrm{pr}_{2}\right)$ has the inverse $\left(\mathrm{r} \circ \mathrm{pr}_{2} ; \mathrm{pr}_{2}\right)$. We also have

$$
\begin{aligned}
\left(g_{0} \cdot g\right) \cdot g_{1} & =\mathrm{s}(g) \cdot g_{1} \\
& =\mathrm{s}\left(g_{1}\right)=\mathrm{s}\left(g \cdot g_{1}\right) \\
& =g_{0} \cdot\left(g \cdot g_{1}\right)
\end{aligned}
$$

for all $g_{0} \in \mathrm{G}^{0}, g, g_{1} \in \mathrm{G}^{1}$ with $g_{0}=\mathrm{r}(g)$ and $\mathrm{s}(g)=\mathrm{r}\left(g_{1}\right)$. The property (2) is done. So we have a right $\mathrm{G}$-action. It is a sheaf because the anchor map is a cover. The left case is similar. If $\mathrm{G}$ is a groupoid then there is a left $\mathrm{G}$-sheaf $\left(\mathrm{G}^{0} ; \mathrm{r} \circ \mathrm{pr}_{1} ; \mathrm{id}_{\mathrm{G}^{0}}\right)$.

Proposition 5.22. $\left(\mathrm{G}^{0} ; \mathrm{s} \circ \mathrm{pr}_{2} ; \mathrm{id}_{\mathrm{G}^{0}}\right)$ is a final object in $\mathcal{C}(\mathrm{G}), \mathcal{C}_{\mathcal{F}_{p}}(\mathrm{G})$ and $\mathcal{C}_{\mathcal{F}}(\mathrm{G})$.

Proof. Let $\left(\mathrm{X} ; \mathrm{m}_{\mathbf{X}} ; \mathrm{s}_{\mathbf{X}}\right)$ be any right $\mathrm{G}$-action. The anchor map $\mathrm{s}_{\mathbf{X}}: \mathrm{X} \rightarrow \mathrm{G}^{0}$ is a right G-map because $\mathrm{s}_{\mathrm{X}}=\mathrm{id}_{\mathrm{G}^{0}} \circ \mathrm{sX}_{\mathrm{X}}$ and $\mathrm{s}_{\mathrm{X}} \circ \mathrm{m}_{\mathrm{X}}=\mathrm{s} \circ \mathrm{pr}_{2}=\mathrm{s} \circ \mathrm{pr}_{2} \circ\left(\mathrm{s}_{\mathrm{X}} \circ \mathrm{pr}_{2} ; \mathrm{pr}_{2}\right)$. Also if any arrow $f: \mathrm{Y} \rightarrow \mathrm{G}^{0}$ is a right $\mathrm{G}$-map then $\mathrm{s}_{\mathrm{Y}}=\mathrm{id}_{\mathrm{G}^{0}} \circ f$. Therefore, any right $\mathrm{G}$-map to $\mathrm{G}^{0}$ is an anchor map. Hence $\left(\mathrm{G}^{0} ; \mathrm{s} \circ \mathrm{pr}_{2} ; \mathrm{id}_{\mathrm{G}^{0}}\right)$ is a final object in $\mathcal{C}(\mathrm{G})$. It is a final object in $\mathcal{C}_{\mathcal{F}_{p}}(\mathrm{G})$ and $\mathcal{C}_{\mathcal{F}}(\mathrm{G})$ too because of Remark 5.15

Example 5.23. Let $f: \mathrm{X} \rightarrow \mathrm{Y}$ be an arrow. If the object $\mathrm{Y}$ is viewed as a 0 -groupoid as in Example 3.14 then there is a right $\mathrm{Y}$-action $\left(\mathrm{X} ; \mathrm{pr}_{1} ; f\right)$. All required properties of the action are clearly satisfied. A Y-map between the $\mathrm{Y}$-actions $\left(\mathrm{X}_{1} ; \mathrm{pr}_{1} ; f_{1}\right)$ and $\left(\mathrm{X}_{2} ; \mathrm{pr}_{1} ; f_{2}\right)$ is an arrow $g: \mathrm{X}_{1} \rightarrow \mathrm{X}_{2}$ with $f_{2} \circ g=f_{1}$. Thus the category of $\mathrm{Y}$-actions $\mathcal{C}(Y)$ is the slice category $\mathcal{C} \downarrow \mathrm{Y}$ of objects in $\mathcal{C}$ over $\mathrm{Y}$. 
Example 5.24. If $\mathrm{G}=\left(\mathrm{G}^{0} ; \mathrm{G}^{1} ; \mathrm{r} ; \mathrm{s} ; \mathrm{m}\right)$ is a groupoid then there is a right $\mathrm{G}$-sheaf $\left(\mathrm{G}^{1} ; \mathrm{m} ; \mathrm{s}\right)$. All required properties of an action are clearly satisfied. Call this action the right translation action. Analogously, we have the left translation G-sheaf $\left(\mathrm{G}^{1} ; \mathrm{m} ; \mathrm{r}\right)$.

Example 5.25. Let $\left(\mathrm{X} ; \mathrm{m}_{\mathrm{X}} ; \mathrm{s}_{\mathrm{X}}\right)$ and $\left(\mathrm{Y} ; \mathrm{m}_{\mathrm{Y}} ; \mathrm{s}_{\mathrm{Y}}\right)$ be right $\mathrm{G}$-actions. If the object

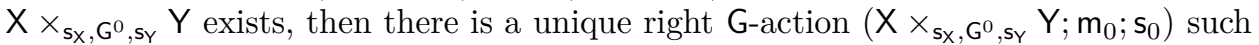
that both coordinate projections are G-maps. Call this action the fibre product

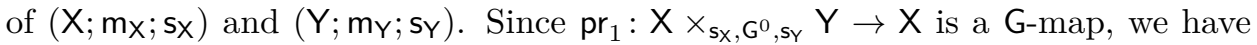
$\mathrm{s}_{0}=\mathrm{s}_{\mathrm{X}} \circ \mathrm{pr}_{1}$. Elementwise $\mathrm{s}_{0}(x ; y)=\mathrm{s}_{\mathbf{X}}(x)$ for all $x \in \mathrm{X}, y \in \mathrm{Y}, g \in \mathrm{G}^{1}$ with $\mathrm{s}_{\mathrm{X}}(x)=\mathrm{s}_{\mathrm{Y}}(y)$. Hence the anchor map $\mathrm{s}_{0}$ is defined uniquely. For the same reason,

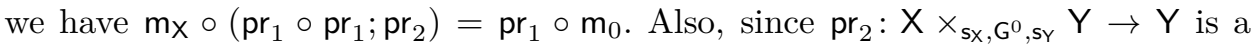
G-map, we have $\mathrm{m}_{\mathrm{Y}} \circ\left(\mathrm{pr}_{2} \circ \mathrm{pr}_{1} ; \mathrm{pr}_{2}\right)=\mathrm{pr}_{2} \circ \mathrm{m}_{0}$. Therefore, the action map $\mathrm{m}_{0}$ is defined uniquely and $\mathrm{m}_{0}=\left(\mathrm{m}_{\mathrm{X}} \circ\left(\mathrm{pr}_{1} \circ \mathrm{pr}_{1} ; \mathrm{pr}_{2}\right) ; \mathrm{m}_{\mathrm{Y}} \circ\left(\mathrm{pr}_{2} \circ \mathrm{pr}_{1} ; \mathrm{pr}_{2}\right)\right)$. Elementwise, $(x ; y) \cdot g=(x \cdot g ; y \cdot g)$ for all $x \in \mathrm{X}, y \in \mathrm{Y}, g \in \mathrm{G}^{1}$ with $\mathrm{sX}(x)=\mathrm{sY}(y)=\mathrm{r}(g)$. We

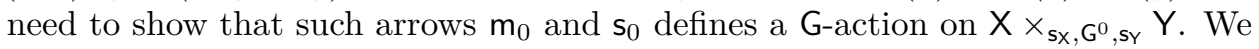
have

$$
\begin{aligned}
\mathrm{s}_{0}((x ; y) \cdot g) & =\mathrm{s}_{0}(x \cdot g ; y \cdot g) \\
& =\mathrm{sx}_{\mathbf{X}}(x \cdot g) \\
& =\mathbf{s}(g)
\end{aligned}
$$

for all $x \in \mathrm{X}, y \in \mathrm{Y}, g \in \mathrm{G}^{1}$ with $\mathrm{s}_{\mathbf{X}}(x)=\mathrm{sY}_{\mathbf{Y}}(y)=\mathrm{r}(g)$. We have a property (1). The property (2) holds because

$$
\begin{aligned}
((x ; y) \cdot g) \cdot g_{1} & =(x \cdot g ; y \cdot g) \cdot g_{1} \\
& =\left((x \cdot g) \cdot g_{1} ;(y \cdot g) \cdot g_{1}\right) \\
& =\left(x \cdot\left(g \cdot g_{1}\right) ; y \cdot\left(g \cdot g_{1}\right)\right) \\
& =(x ; y) \cdot\left(g \cdot g_{1}\right)
\end{aligned}
$$

for all $x \in \mathrm{X}, y \in \mathrm{Y}, g, g_{1} \in \mathrm{G}^{1}$ with $\mathrm{s}_{\mathrm{X}}(x)=\mathrm{s}_{\mathrm{Y}}(y)=\mathrm{r}(g)$ and $\mathrm{s}(g)=\mathrm{r}\left(g_{1}\right)$. We also have

$$
\begin{aligned}
(x ; y) \cdot 1_{\mathrm{s}_{0}(x, y)} & =\left(x \cdot 1_{\mathrm{s}_{0}(x, y)} ; y \cdot 1_{\mathrm{s}_{0}(x, y)}\right) \\
& =\left(x \cdot 1_{\mathrm{s}_{\mathrm{X}}(x)} ; y \cdot 1_{\mathrm{s}_{\mathrm{Y}}(y)}\right) \\
& =(x ; y)
\end{aligned}
$$

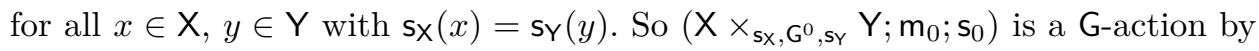
Lemma 5.7

Example 5.26. Let $\mathrm{F}: \mathrm{G} \rightarrow \mathrm{H}$ be a functor between groupoids. There is a left G-sheaf $\left(X ; m_{X} ; r_{X}\right)$, where $X=G^{0} \times{ }_{F^{0}, H^{0}, r_{H}} H^{1}, r_{X}=p r_{1}: G^{0} \times{ }_{F^{0}, H^{0}, r_{H}} H^{1} \rightarrow G^{0}$ and

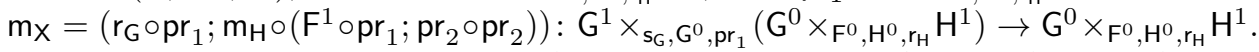
Elementwise, $\left.g \cdot\left(g_{0} ; h\right)\right)=\left(\mathrm{r}_{\mathrm{G}}(g) ; \mathrm{F}^{1}(g) \cdot h\right)$, for all $g_{0} \in \mathrm{G}^{0}, g \in \mathrm{G}^{1}, h \in \mathrm{H}^{1}$ with $\mathrm{s}_{\mathrm{G}}(g)=g_{0}$ and $\mathrm{F}^{0}\left(g_{0}\right)=\mathrm{r}_{\mathrm{H}}(h)$. The object $\mathrm{X}$ exists because $\mathrm{r}_{\mathrm{H}}: \mathrm{H}^{1} \rightarrow \mathrm{H}^{0}$ is a cover. Since it is a splitting cover, the anchor map $r_{X}=p r_{1}: G^{0} \times_{F^{0}, H^{0}, r_{H}} H^{1} \rightarrow G^{0}$ is a cover by Corollary 2.7. We have $\mathrm{r}_{\mathrm{X}}\left(g \cdot\left(g_{0} ; h\right)\right)=\mathrm{pr}_{1}\left(\mathrm{r}_{\mathrm{G}}(g) ; \mathrm{F}^{1}(g) \cdot h\right)=\mathrm{r}_{\mathrm{G}}(g)$. That is the property (1) in Definition $5.8 \mathrm{~m}_{\mathrm{X}}$ commutes with $\mathrm{m}_{\mathrm{G}}$ because

$$
\begin{aligned}
\left(g \cdot g_{1}\right) \cdot\left(g_{0} ; h\right) & =\left(\mathrm{r}_{\mathrm{G}}\left(g \cdot g_{1}\right) ; \mathrm{F}^{1}\left(g \cdot g_{1}\right) \cdot h\right) \\
& =\left(\mathrm{r}_{\mathrm{G}}(g) ; \mathrm{F}^{1}(g) \cdot \mathrm{F}^{1}\left(g_{1}\right) \cdot h\right) \\
& =g \cdot\left(\mathrm{s}_{\mathrm{G}}(g) ; \mathrm{F}^{1}\left(g_{1}\right) \cdot h\right) \\
& =g \cdot\left(\mathrm{r}_{\mathrm{G}}\left(g_{1}\right) ; \mathrm{F}^{1}\left(g_{1}\right) \cdot h\right) \\
& =g \cdot\left(g_{1} \cdot\left(\mathrm{s}_{\mathrm{G}}(g) ; h\right)\right) \\
& =g \cdot\left(g_{1} \cdot\left(g_{0} ; h\right)\right)
\end{aligned}
$$


for all $g_{0} \in \mathrm{G}^{0}, g, g_{1} \in \mathrm{G}^{1}, h \in \mathrm{H}^{1}$ with $\mathrm{s}_{\mathrm{G}}\left(g_{1}\right)=g_{0}, \mathrm{r}_{\mathrm{G}}\left(g_{1}\right)=\mathrm{s}_{\mathrm{G}}(g)$ and $\mathrm{F}^{0}\left(g_{0}\right)=$ $\mathrm{r}_{\mathrm{H}}(h)$. We also have

$$
\begin{aligned}
1_{\mathrm{s}_{0}\left(g_{0} ; h\right)} \cdot\left(g_{0} ; h\right) & =1_{g_{0}} \cdot\left(g_{0} ; h\right) \\
& =\left(\mathrm{r}_{\mathrm{G}}\left(1_{g_{0}}\right) ; \mathrm{F}^{1}\left(1_{g_{0}}\right) \cdot h\right) \\
& =\left(g_{0} ; 1_{\mathrm{F}^{0}\left(g_{0}\right)} \cdot h\right) \\
& =\left(g_{0} ; 1_{\mathrm{r}_{\mathrm{H}}(h)} \cdot h\right) \\
& =\left(g_{0} ; h\right)
\end{aligned}
$$

for all $g_{0} \in \mathrm{G}^{0}, h \in \mathrm{H}^{1}$ with $\mathrm{F}^{0}\left(g_{0}\right)=\mathrm{r}_{\mathrm{H}}(h)$. So we have all required properties in Remark 5.11 and, therefore, the triple $\left(\mathrm{X} ; \mathrm{m}_{\mathrm{X}} ; \mathrm{rX}_{\mathrm{X}}\right)$ defines a G-sheaf.

Example 5.27. Let $\mathrm{G}$ be the fibre of the partial groupoid fibration $\mathrm{F}: \mathrm{L} \rightarrow \mathrm{H}$. There is a left $G$-sheaf $\left(L^{1} ; m ; r\right)$, where $r=r_{L}$ and $m=m_{L} \circ\left(p_{1} \circ p_{1} ; p_{2}\right)$. Since the element $g \in \mathrm{G}^{1}$ can be understood as an element in $\mathrm{L}^{1}$, the action map can be defined elementwise by $g \cdot l=g \cdot l$, for all $g \in \mathrm{G}^{1}, l \in \mathrm{L}^{1}$ with $\mathrm{s}_{\mathrm{G}}(g)=\mathrm{r}_{\mathrm{L}}(l)$. All required properties are clearly satisfied by Remark 5.11. Therefore, $\left(\mathrm{L}^{1} ; \mathrm{m} ; \mathrm{r}\right)$ is a left G-action.

Remark 5.28. There is also a right $\mathrm{G}$-sheaf as in Example 5.27, namely, $\left(\mathrm{L}^{1} ; \mathrm{m}_{\mathrm{L}} \circ\right.$ $\left.\left(\mathrm{pr}_{1} ; \mathrm{pr}_{1} \circ \mathrm{pr}_{2}\right) ; \mathrm{s}_{\mathrm{L}}\right)$. The proof is absolutely similar.

\subsection{Transformation groupoids.}

Definition 5.29. Let $\left(X ; m_{X} ; s_{X}\right)$ be a right $G$-action. There is a transformation groupoid $X \rtimes G$ with $X$ as objects and $X \times_{s_{x}, G^{0}, r} G^{1}$ as arrows. The range map $r_{(X \rtimes G)}$ and the source map $s_{(X \rtimes G)}$ are $\operatorname{pr}_{1}$ and $m_{X}$, respectively. The multiplication map $\mathrm{m}_{(\mathrm{X} \rtimes \mathrm{G})}$ is

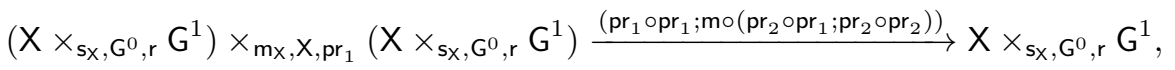

defined elementwise by $(x ; g) \cdot\left(x_{1} ; g_{1}\right)=\left(x ; g \cdot g_{1}\right)$, for all $x, x_{1} \in \mathrm{X}, g, g_{1} \in \mathrm{G}^{1}$ with $\mathrm{s} \mathrm{x}(x)=\mathrm{r}(g), \mathrm{sX}_{\mathbf{X}}\left(x_{1}\right)=\mathrm{r}\left(g_{1}\right)$ and $x \cdot g=x_{1}$.

Lemma 5.30. The data in Definition 5.29 defines a groupoid.

Proof. The source map $\mathrm{m}_{\mathrm{X}}$ is a cover by Lemma 5.6. The range map $\mathrm{pr}_{1}$ is a cover because it is a pull-back of $r$ : $G^{1} \rightarrow G^{0}$, which splits by $u: G^{0} \rightarrow G^{1}$, so that Corollary 2.7 works. So condition (1) in Definition 3.1 holds.

Also we have

$$
\begin{aligned}
\mathrm{s}_{(\mathrm{X} \rtimes \mathrm{G})}\left((x ; g) \cdot\left(x_{1} ; g_{1}\right)\right) & =\mathrm{m}_{(\mathrm{X} \rtimes \mathrm{G})}\left(x ; g \cdot g_{1}\right) \\
& =x \cdot\left(g \cdot g_{1}\right)=(x \cdot g) \cdot g_{1} \\
& =x_{1} \cdot g_{1} \\
& =\mathbf{s}_{(\mathrm{X} \rtimes \mathrm{G})}\left(x_{1} ; g_{1}\right)
\end{aligned}
$$

for all $x, x_{1} \in \mathrm{X}, g, g_{1} \in \mathrm{G}^{1}$ with $\mathrm{s}_{\mathbf{X}}(x)=\mathrm{r}(g), \mathrm{s}_{\mathbf{X}}\left(x_{1}\right)=\mathrm{r}\left(g_{1}\right)$ and $x \cdot g=x_{1}$. So the condition $[(2)$ is satisfied.

We also have to show that the multiplication map $\mathrm{m}_{(\mathrm{X} \rtimes \mathrm{G})}$ is associative. We have

$$
\begin{aligned}
\left((x ; g) \cdot\left(x_{1} ; g_{1}\right)\right) \cdot\left(x_{2} ; g_{2}\right) & =\left(x ; g \cdot g_{1}\right) \cdot\left(x_{2} ; g_{2}\right) \\
& =\left(x ;\left(g \cdot g_{1}\right) \cdot g_{2}\right) \\
& =\left(x ; g \cdot\left(g_{1} \cdot g_{2}\right)\right) \\
& =(x ; g) \cdot\left(x_{1} ; g_{1} \cdot g_{2}\right) \\
& =(x ; g) \cdot\left(\left(x_{1} ; g_{1}\right) \cdot\left(x_{2} ; g_{2}\right)\right)
\end{aligned}
$$

for all $x, x_{1}, x_{2} \in \mathrm{X}, g, g_{1}, g_{2} \in \mathrm{G}^{1}$ with $\mathrm{s}_{\mathbf{X}}(x)=\mathrm{r}(g), \mathrm{s}_{\mathbf{X}}\left(x_{1}\right)=\mathrm{r}\left(g_{1}\right), \mathrm{s}_{\mathbf{X}}\left(x_{2}\right)=\mathrm{r}\left(g_{2}\right)$, $x \cdot g=x_{1}$ and $x_{1} \cdot g_{1}=x_{2}$. So the multiplication map $\mathrm{m}_{(\mathrm{X} \rtimes \mathrm{G})}$ is associative. 
We proved the properties (1) (2) and $(3)$ in Definition 3.1. The next step is to construct the arrows $\mathrm{u}_{(\mathrm{X} \rtimes \mathrm{G})}:(\mathrm{X} \rtimes \mathrm{G})^{0} \rightarrow(\mathrm{X} \rtimes \mathrm{G})^{1}$ and $\mathrm{i}_{(\mathrm{X} \rtimes \mathrm{G})}:(\mathrm{X} \rtimes \mathrm{G})^{1} \rightarrow(\mathrm{X} \rtimes \mathrm{G})^{1}$ and prove all properties in Proposition 3.8 , which then allows to use Proposition 3.11

We know from the proof of Lemma 5.6 that there is a well-defined arrow

$$
\mathrm{u}_{(\mathrm{X} \rtimes \mathrm{G})}: \mathrm{X} \stackrel{\left(\mathrm{idx}_{\mathrm{C}} \text { uosx }\right)}{\longrightarrow} \mathrm{X} \times_{\mathrm{sX}_{\mathrm{X}}, \mathrm{G}^{0}, \mathrm{r}} \mathrm{G}^{1}, \quad x \mapsto\left(x ; 1_{\mathrm{sX}(x)}\right) .
$$

We have the following: $\mathrm{s}_{(\mathrm{X} \rtimes \mathrm{G})}\left(\mathrm{u}_{(\mathrm{X} \rtimes \mathrm{G})}(x)\right)=\mathrm{s}_{(\mathrm{X} \rtimes \mathrm{G})}\left(x ; 1_{\mathrm{SX}_{X}(x)}\right)=x \cdot 1_{\mathrm{SX}_{X}(x)}=x$ and $\mathrm{r}_{(\mathrm{X} \rtimes \mathrm{G})}\left(\mathrm{u}_{(\mathrm{X} \rtimes \mathrm{G})}(x)\right)=\mathrm{r}_{(\mathrm{X} \rtimes \mathrm{G})}\left(x ; 1_{\mathrm{s}_{\mathrm{X}}(x)}\right)=x$ for all $x \in \mathrm{X}$. So (1) in Propesition 3.11 is done. We also have

$$
\begin{aligned}
1_{\mathrm{r}_{(\mathrm{X} \rtimes \mathrm{G})}(x ; g)} \cdot(x ; g) & =1_{x} \cdot(x ; g) \\
& =\left(x ; 1_{\mathrm{s}_{\mathrm{X}}(x)}\right) \cdot(x ; g) \\
& =\left(x ; 1_{\mathrm{r}(g)} \cdot g\right) \\
& =(x ; g)
\end{aligned}
$$

and

$$
\begin{aligned}
(x ; g) \cdot 1_{\mathrm{s}_{(\mathrm{X} \rtimes \mathrm{G})}(x ; g)} & =(x ; g) \cdot 1_{x \cdot g} \\
& =(x ; g) \cdot\left(x \cdot g ; 1_{\mathrm{s}_{\mathbf{X}}(x \cdot g)}\right) \\
& =\left(x ; g \cdot 1_{\mathrm{s}(g)}\right) \\
& =(x ; g)
\end{aligned}
$$

for all $x \in \mathrm{X}, g \in \mathrm{G}^{1}$ with $\mathrm{sX}_{\mathrm{X}}(x)=\mathrm{r}(g)$. So condition $(2)$ is satisfied.

Now consider the arrow

$$
\mathrm{i}_{(\mathrm{X} \rtimes \mathrm{G})}: \mathrm{X} \times_{\mathrm{s}_{\mathrm{X}}, \mathrm{G}^{0}, \mathrm{r}} \mathrm{G}^{1} \stackrel{\left(\mathrm{m}_{\mathrm{X}} ; \mathrm{iopr}_{2}\right)}{\longrightarrow} \mathrm{X} \times_{\mathrm{sX}_{\mathrm{X}}, \mathrm{G}^{0}, \mathrm{r}} \mathrm{G}^{1}, \quad(x ; g) \mapsto\left(x \cdot g ; g^{-1}\right) .
$$

It is well-defined because $\mathrm{sx}_{\mathbf{X}}(x \cdot g)=\mathrm{s}(g)=\mathrm{r}\left(g^{-1}\right)$. We have

$$
\begin{aligned}
\mathrm{s}_{(\mathrm{X} \rtimes \mathrm{G})}\left(\mathrm{i}_{(\mathrm{X} \rtimes \mathrm{G})}(x ; g)\right) & =\mathrm{s}_{(\mathrm{X} \rtimes \mathrm{G})}\left(x \cdot g ; g^{-1}\right) \\
& =(x \cdot g) \cdot g^{-1} \\
& =x \cdot\left(g \cdot g^{-1}\right) \\
& =x \cdot 1_{\mathrm{r}(g)} \\
& =x \cdot 1_{\mathrm{s}_{X}(x)} \\
& =x \\
& =\mathrm{r}_{(\mathrm{X} \rtimes \mathrm{G})}(x ; g)
\end{aligned}
$$

and

$$
\begin{aligned}
\mathrm{r}_{(\mathrm{X} \rtimes \mathrm{G})}\left(\mathrm{i}_{(\mathrm{X} \rtimes \mathrm{G})}(x ; g)\right) & =\mathrm{r}_{(\mathrm{X} \rtimes \mathrm{G})}\left(x \cdot g ; g^{-1}\right) \\
& =x \cdot g \\
& =\mathrm{s}_{(\mathrm{X} \rtimes \mathrm{G})}(x ; g)
\end{aligned}
$$

for all $x \in \mathrm{X}, g \in \mathrm{G}^{1}$ with $\mathrm{sx}_{\mathbf{X}}(x)=\mathrm{r}(g)$. So all conditions in Proposition (3) hold.

We also have

$$
\begin{aligned}
\mathrm{i}_{(\mathrm{X} \rtimes \mathrm{G})}(x ; g) \cdot(x ; g) & =\left(x \cdot g ; g^{-1}\right) \cdot(x ; g) \\
& =\left(x \cdot g ; g^{-1} \cdot g\right) \\
& =\left(x \cdot g ; 1_{\mathrm{s}(g)}\right) \\
& =\left(x \cdot g ; 1_{\mathrm{s} \times}(x \cdot g)\right) \\
& =1_{x \cdot g} \\
& =\mathrm{u}_{(\mathrm{X} \rtimes \mathrm{G})}\left(\mathrm{s}_{(\mathrm{X} \rtimes \mathrm{G})}(x ; g)\right)
\end{aligned}
$$


and

$$
\begin{aligned}
(x ; g) \cdot \mathrm{i}_{(\mathrm{X} \rtimes \mathrm{G})}(x ; g) & =(x ; g) \cdot\left(x \cdot g ; g^{-1}\right) \\
& =\left(x ; g \cdot g^{-1}\right) \\
& =\left(x ; 1_{\mathrm{r}(g)}\right) \\
& =\left(x ; 1_{\mathrm{s}_{X}(x)}\right) \\
& =1_{x} \\
& =\mathrm{u}_{(\mathrm{X} \rtimes \mathrm{G})}\left(\mathrm{r}_{(\mathrm{X} \rtimes \mathrm{G})}(x ; g)\right)
\end{aligned}
$$

for all $x \in \mathrm{X}, g \in \mathrm{G}^{1}$ with $\mathrm{sx}(x)=\mathrm{r}(g)$.

We proved all required properties for Proposition 3.11.Therefore, we can deduce that the transformation groupoid is a well-defined groupoid.

We can define the transformation groupoid of a left action analogously. Let $\left(\mathrm{X} ; \mathrm{m}_{\mathrm{X}} ; r_{\mathrm{X}}\right)$ be a left $\mathrm{G}$-action. There is a transformation groupoid $\mathrm{G} \ltimes \mathrm{X}$ with $\mathrm{X}$ as objects and $\mathrm{G}^{1} \times_{\mathrm{s}, \mathrm{G}^{0}, \mathrm{r}_{\mathbf{X}}} \mathrm{X}$ as arrows. The range and source maps are $\mathrm{m}_{\mathbf{X}}$ and $\mathrm{pr}_{2}$, respectively. The multiplication map is

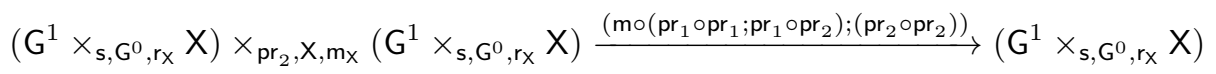

defined elementwise by $(g ; x) \cdot\left(g_{1} ; x_{1}\right)=\left(g \cdot g_{1} ; x_{1}\right)$, for all $x, x_{1} \in \mathrm{X}, g, g_{1} \in \mathrm{G}^{1}$ with $\mathbf{s}(g)=\mathbf{r}_{\mathbf{X}}(x), \mathbf{s}\left(g_{1}\right)=\mathbf{r}_{\mathbf{X}}\left(x_{1}\right)$ and $x=g_{1} \cdot x_{1}$.

Transformation groupoids give an important example of a groupoid covering.

Example 5.31. Let $\left(\mathrm{X} ; \mathrm{m}_{\mathbf{X}} ; \mathrm{r}_{\mathbf{X}}\right)$ be a left $\mathrm{G}$-action. There is a groupoid covering from the transformation groupoid of $\left(X ; m_{X} ; r_{X}\right)$ to $G$, which is $p_{1}: G^{1} \times_{s, G^{0}, r_{X}} X \rightarrow G^{1}$ on arrows and $r_{X}: X \rightarrow G^{0}$ on objects. These maps clearly intertwine the source and range maps. $\mathrm{pr}_{1}$ intertwines the multiplication maps because

$$
\begin{aligned}
\operatorname{pr}_{1}\left((g ; x) \cdot\left(g_{1} ; x_{1}\right)\right) & =\operatorname{pr}_{1}\left(g \cdot g_{1} ; x_{1}\right) \\
& =g \cdot g_{1} \\
& =\operatorname{pr}_{1}(g ; x) \cdot \operatorname{pr}_{1}\left(g_{1} ; x_{1}\right) .
\end{aligned}
$$

This functor is a groupoid covering because the arrow (4.1) in this case is $\left(\mathrm{pr}_{1} ; \mathrm{pr}_{2}\right)$, which is the identity arrow on $G^{1} \times_{s, G^{0}, r x} X$. Analogously, for a right action, we have a groupoid covering $F: X \rtimes G \rightarrow G$, which is the arrow $\mathrm{pr}_{2}: X \times_{s_{X}, G^{0}, r} G^{1} \rightarrow G^{1}$ on arrows and $\mathrm{s}_{X}: \mathrm{X} \rightarrow \mathrm{G}^{0}$ on objects.

Proposition 5.32. Let $\left(\mathrm{X}, \mathrm{m}_{\mathrm{X}}, \mathrm{s}_{\mathrm{X}}\right)$ be a $\mathrm{G}$-action. An action of the transformation groupoid $\mathrm{X} \rtimes \mathrm{G}$ on an object $\mathrm{Y}$ is equivalent to an action of $\mathrm{G}$ on $\mathrm{Y}$ together with a $\mathrm{G}-$ map $f: \mathrm{Y} \rightarrow \mathrm{X}$. Furthermore, the following groupoids are isomorphic:

$$
\mathrm{Y} \rtimes(\mathrm{X} \rtimes \mathrm{G}) \cong \mathrm{Y} \rtimes \mathrm{G} .
$$

A map $\mathrm{Y} \rightarrow \mathrm{Z}$ is $\mathrm{X} \rtimes \mathrm{G}$-invariant if and only if it is $\mathrm{G}$-invariant, and a map between two $\mathrm{X} \rtimes \mathrm{G}$-actions is an $\mathrm{X} \rtimes \mathrm{G}$-map if and only if it is a $\mathrm{G}-$ map over $\mathrm{X}$.

Proof. Let $\left(\mathrm{Y} ; \mathrm{m}_{\mathrm{Y}} ; \mathrm{s}_{\mathrm{Y}}\right)$ be a $\mathrm{G}$-action and let $f: \mathrm{Y} \rightarrow \mathrm{X}$ be a $\mathrm{G}-\mathrm{map}$. There is an $\mathrm{X} \rtimes \mathrm{G}$-action $\left(\mathrm{Y} ; \hat{\mathrm{m}}_{\mathrm{Y}} ; \hat{\mathrm{s}}_{\mathrm{Y}}\right)$ where $\hat{\mathrm{s}}_{\mathrm{Y}}=f$ and $\hat{\mathrm{m}}_{\mathrm{Y}}=\mathrm{m}_{\mathrm{Y}} \circ\left(\mathrm{pr}_{1} ; \mathrm{pr}_{2} \circ \mathrm{pr}_{2}\right)$. Elementwise, $y \cdot(x ; g)=y \cdot g$ for all $x \in \mathrm{X}, y \in \mathrm{Y}, g \in \mathrm{G}^{1}$ with $x=f(y)$ and $\mathrm{sX}(x)=\mathrm{r}(g)$. We have

$$
\begin{aligned}
\hat{\mathrm{s}}_{\mathbf{Y}}(y \cdot(x ; g)) & =f(y \cdot g) \\
& =f(y) \cdot g \\
& =x \cdot g \\
& =\mathrm{s}_{(\mathrm{X} \rtimes \mathrm{G})}(x ; g)
\end{aligned}
$$


for all $x \in \mathrm{X}, y \in \mathrm{Y}, g \in \mathrm{G}^{1}$ with $x=f(y)$ and $\mathrm{s}_{\mathbf{X}}(x)=\mathrm{r}(g)$. So we have the condition (1) in Definition 5.1. Also

$$
\begin{aligned}
(y \cdot(x ; g)) \cdot\left(x_{1} ; g_{1}\right) & =(y \cdot g) \cdot\left(x_{1} ; g_{1}\right) \\
& =(y \cdot g) \cdot g_{1} \\
& =y \cdot\left(g \cdot g_{1}\right) \\
& =y \cdot\left(x ; g \cdot g_{1}\right) \\
& =y \cdot\left((x ; g) \cdot\left(x_{1} ; g_{1}\right)\right)
\end{aligned}
$$

for all $x, x_{1} \in \mathrm{X}, y \in \mathrm{Y}, g, g_{1} \in \mathrm{G}^{1}$ with $x=f(y), \mathrm{s}_{\mathbf{X}}(x)=\mathrm{r}(g), \mathrm{s}_{\mathbf{X}}\left(x_{1}\right)=\mathrm{r}\left(g_{1}\right)$ and $x_{1}=x \cdot g$. Therefore, the action map $\hat{\mathrm{m}}_{\mathrm{Y}}$ commutes with the multiplication map $\mathrm{m}_{\mathbf{x} \rtimes \mathrm{G}}$. We also have

$$
\begin{aligned}
y \cdot 1_{\hat{\mathrm{S}}(y)} & =y \cdot 1_{f(y)} \\
& =y \cdot\left(f(y) ; 1_{\mathrm{s}_{\mathbf{X}}(f(y))}\right) \\
& =y \cdot 1_{\mathrm{SY}(y)} \\
& =y
\end{aligned}
$$

for all $y \in \mathrm{Y}$. Therefore, $\left(\mathrm{Y} ; \hat{\mathrm{m}}_{\mathrm{Y}} ; \hat{\mathrm{s}}_{\mathrm{Y}}\right)$ is an $\mathrm{X} \rtimes \mathrm{G}$-action by Lemma 5.7 .

Conversely, if $\left(\mathrm{Y} ; \hat{\mathrm{m}}_{\mathrm{Y}} ; \hat{\mathrm{s}}_{\mathrm{Y}}\right)$ is a right $\mathrm{X} \rtimes \mathrm{G}$-action then there is a right $\mathrm{G}$-action $\left(\mathrm{Y} ; \overline{\mathrm{m}_{Y}} ; \overline{\mathrm{s}_{Y}}\right)$, such that the anchor map $\hat{s}_{Y}: \mathrm{Y} \rightarrow \mathrm{X}$ is a $\mathrm{G}$-map, where $\overline{\mathrm{s}_{Y}}=\mathrm{s}_{\mathrm{X}} \circ \hat{\mathrm{s}} \mathrm{Y}$ and $\overline{\mathrm{m}_{\mathrm{Y}}}=\hat{\mathrm{m}}_{\mathrm{Y}} \circ\left(\mathrm{pr}_{1} ;\left(\hat{\mathrm{s}}_{\mathrm{Y}} \circ \mathrm{pr}_{1} ; \mathrm{pr}_{2}\right)\right)$. Elementwise, $\overline{\mathrm{s}_{\mathrm{Y}}}(y)=\mathrm{s}_{\mathrm{X}}\left(\hat{\mathrm{s}}_{\mathrm{Y}}(y)\right)$ and $y \cdot g=y \cdot\left(\hat{\mathrm{s}}_{\mathrm{Y}}(y) ; g\right)$ for all $y \in \mathrm{Y}, g \in \mathrm{G}^{1}$ with $\mathrm{s}_{\mathbf{X}}\left(\hat{\mathrm{s}}_{\mathbf{Y}}(y)\right)=\mathrm{r}(g)$.

We are going to prove the conditions (1) and (2) in Definition 5.1. We have

$$
\begin{aligned}
\overline{\mathrm{s}_{\mathbf{Y}}}(y \cdot g) & =\mathrm{s}_{\mathbf{X}}\left(\hat{\mathrm{s}}_{\mathbf{Y}}\left(y \cdot\left(\hat{\mathrm{s}}_{\mathbf{Y}}(y) ; g\right)\right)\right) \\
& \left.=\mathrm{s}_{\mathbf{X}}\left(\mathrm{s}_{\mathbf{X} \rtimes \mathrm{G}}\right)\left(\hat{\mathrm{s}}_{\mathbf{Y}}(y) ; g\right)\right) \\
& =\mathrm{s}_{\mathbf{X}}\left(\hat{\mathrm{s}}_{\mathbf{Y}}(y) \cdot g\right) \\
& =\mathrm{s}(g)
\end{aligned}
$$

for all $y \in \mathrm{Y}, g \in \mathrm{G}^{1}$ with $\mathrm{s}_{\mathrm{X}}\left(\hat{\mathrm{s}}_{\mathrm{Y}}(y)\right)=\mathrm{r}(g)$. The condition (1) is done. We also have

$$
\begin{aligned}
(y \cdot g) \cdot g_{1} & =\left(y \cdot\left(\hat{\mathrm{s}}_{\mathbf{Y}}(y) ; g\right)\right) \cdot g_{1} \\
& =\left(y \cdot\left(\hat{\mathrm{s}}_{\mathbf{Y}}(y) ; g\right)\right) \cdot\left(\hat{\mathrm{s}}_{\mathbf{Y}}\left(y \cdot\left(\hat{\mathrm{s}}_{\mathbf{Y}}(y) ; g\right) ; g_{1}\right)\right. \\
& =\left(y \cdot\left(\hat{\mathrm{s}}_{\mathbf{Y}}(y) ; g\right)\right) \cdot\left(\mathbf{s}_{(\mathrm{X} \rtimes \mathrm{G}}\left(\hat{\mathrm{s}}_{\mathbf{Y}}(y) ; g\right) ; g_{1}\right) \\
& =\left(y \cdot\left(\hat{\mathrm{s}}_{\mathbf{Y}}(y) ; g\right)\right) \cdot\left(\hat{\mathrm{s}}_{\mathbf{Y}}(y) \cdot g ; g_{1}\right) \\
& =y \cdot\left(\left(\hat{\mathrm{s}}_{\mathbf{Y}}(y) ; g\right) \cdot\left(\hat{\mathrm{s}}_{\mathbf{Y}}(y) \cdot g ; g_{1}\right)\right) \\
& =y \cdot\left(\hat{\mathrm{s}}_{\mathbf{Y}}(y) ; g \cdot g_{1}\right) \\
& =y \cdot\left(g \cdot g_{1}\right)
\end{aligned}
$$

for all $y \in \mathrm{Y}, g, g_{1} \in \mathrm{G}^{1}$ with $\mathrm{s}_{\mathbf{X}}\left(\hat{\mathrm{s}}_{\mathrm{Y}}(y)\right)=\mathrm{r}(g)$ and $\mathrm{s}(g)=\mathrm{r}\left(g_{1}\right)$. Hence the condition (2) holds. The next step is to check (5.2). We have

$$
\begin{aligned}
y \cdot 1_{\overline{\mathrm{s}_{\mathrm{Y}}}(y)} & =y \cdot\left(\hat{\mathrm{s}}_{\mathrm{Y}}(y) ; 1_{\overline{\mathrm{s}_{\mathbf{Y}}}(y)}\right) \\
& =y \cdot\left(\hat{\mathrm{s}}_{\mathbf{Y}}(y) ; 1_{\mathrm{s}_{\mathrm{X}}\left(\hat{\mathrm{s}}_{\mathrm{Y}}(y)\right)}\right) \\
& =y \cdot 1_{\hat{\mathrm{s}}_{\mathbf{Y}}(y)} \\
& =y
\end{aligned}
$$

for all $y \in \mathrm{Y}$. So we have all properties which are required in Lemma 5.7 Hence $\left(\mathrm{Y} ; \overline{m_{Y}} ; \overline{s_{Y}}\right)$ is a $\mathrm{G}$-action. The arrow $\hat{s}_{Y}: \mathrm{Y} \rightarrow \mathrm{X}$ is a $\mathrm{G}$-map because $\overline{\mathrm{s}_{\mathrm{Y}}}=\mathrm{s}_{\mathrm{X}} \circ \hat{\mathrm{s}}_{\mathrm{Y}}$ and

$$
\begin{aligned}
\hat{\mathrm{s}}_{\mathbf{Y}}(y \cdot g) & =\hat{\mathbf{s}}_{\mathbf{Y}}\left(y \cdot\left(\hat{\mathrm{s}}_{\mathbf{Y}}(y) ; g\right)\right) \\
& =\mathbf{s}_{(\mathbf{X} \boldsymbol{G})}\left(\hat{\mathbf{s}}_{\mathbf{Y}}(y) ; g\right) \\
& =\hat{\mathbf{s}}_{\mathbf{Y}}(y) \cdot g
\end{aligned}
$$


for all $y \in \mathrm{Y}, g \in \mathrm{G}^{1}$ with $\mathrm{s}_{\mathbf{X}}\left(\hat{\mathbf{s}}_{\mathbf{Y}}(y)\right)=\mathrm{r}(g)$. It is clear that these two processes are inverse to each other. So the first part of the lemma is proved.

Consider an arrow $\alpha: \mathrm{Y} \rightarrow \mathrm{Z}$ which is G-invariant. Then

$$
\begin{aligned}
\alpha(y \cdot(x ; g)) & =\alpha(y \cdot g) \\
& =\alpha(y)
\end{aligned}
$$

for all $x \in \mathrm{X}, y \in \mathrm{Y}, g \in \mathrm{G}^{1}$ with $x=\hat{\mathrm{s}}_{\mathrm{Y}}(y)$ and $\mathrm{s}_{\mathrm{X}}(x)=\mathrm{r}(g)$. So $\alpha: \mathrm{Y} \rightarrow \mathrm{Z}$ is $\mathrm{X} \rtimes \mathrm{G}$-invariant. Conversely, if $\alpha: \mathrm{Y} \rightarrow \mathrm{Z}$ is $\mathrm{X} \rtimes \mathrm{G}$-invariant

$$
\begin{aligned}
\alpha(y \cdot g) & =\alpha\left(y \cdot\left(\hat{\mathrm{S}}_{\mathbf{Y}}(y) ; g\right)\right) \\
& =\alpha(y)
\end{aligned}
$$

for all $y \in \mathrm{Y}, g \in \mathrm{G}^{1}$ with $\mathrm{s}_{\mathbf{X}}\left(\hat{\mathrm{s}}_{\mathbf{Y}}(y)\right)=\mathrm{r}(g)$. Hence the arrow $\alpha: \mathrm{Y} \rightarrow \mathrm{Z}$ is $\mathrm{G}$-invariant if and only if it is $\mathrm{X} \rtimes \mathrm{G}$-invariant.

Consider an arrow $\beta: \mathrm{Y}_{1} \rightarrow \mathrm{Y}_{2}$ which is an $\mathrm{X} \rtimes \mathrm{G}$-map. That is, $\hat{\mathrm{s}}_{\mathrm{Y}_{1}}(y)=\hat{\mathrm{s}}_{\mathrm{Y}_{2}}(\beta(y))$ and $\beta(y \cdot(x ; g))=\beta(y) \cdot(x ; g)$ for all $y \in \mathrm{Y}_{1}, x \in \mathrm{X}, g \in \mathrm{G}^{1}$ with $x=\hat{\mathbf{s}}_{\mathrm{Y}_{1}}(y)$ and $\mathrm{s}_{\mathbf{X}}(x)=\mathrm{r}(g)$. We have $\overline{\mathrm{s}_{1}}(y)=\mathrm{s}_{\mathbf{X}}\left(\hat{\mathrm{s}}_{\mathrm{Y}_{1}}(y)\right)=\mathrm{s}_{\mathbf{X}}\left(\hat{\mathrm{s}}_{\mathbf{Y}_{2}}(\beta(y))=\overline{\mathrm{s}_{\mathbf{Y}}}(\beta(y))\right.$ and

$$
\begin{aligned}
\beta(y \cdot g) & =\beta\left(y \cdot\left(\hat{\mathrm{s}}_{\mathbf{Y}_{1}}(y) ; g\right)\right) \\
& =\beta(y) \cdot\left(\hat{\mathrm{s}}_{\mathbf{Y}_{1}}(y) ; g\right) \\
& =\beta(y) \cdot\left(\hat{\mathrm{s}}_{\mathbf{Y}}(\beta(y)) ; g\right) \\
& =\beta(y) \cdot g
\end{aligned}
$$

for all $y \in \mathrm{Y}_{1}, g \in \mathrm{G}^{1}$ with $\mathrm{sX}_{\mathbf{X}}\left(\hat{\mathrm{s}}_{\mathbf{Y}}(y)\right)=\mathrm{r}(g)$. Hence the arrow $\beta: \mathrm{Y}_{1} \rightarrow \mathrm{Y}_{2}$ is a G-map. Conversely, if we have three $\mathrm{G}$-maps $\beta, f_{1}, f_{2}$ such that the following diagram commutes

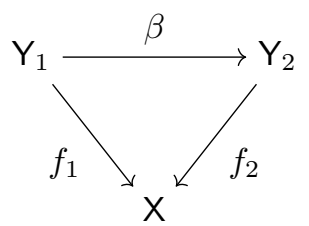

then $\beta: \mathrm{Y}_{1} \rightarrow \mathrm{Y}_{2}$ is an $\mathrm{X} \rtimes \mathrm{G}$-map. This is true because $f_{1}=f_{2} \circ \beta$ and

$$
\begin{aligned}
\beta(y \cdot(x ; g)) & =\beta(y \cdot g) \\
& =\beta(y) \cdot g \\
& =\beta(y) \cdot\left(\hat{\mathrm{s}}_{Y_{2}}(\beta(y)) ; g\right) \\
& =\beta(y) \cdot\left(\hat{\mathrm{s}}_{Y_{1}}(y) ; g\right) \\
& =\beta(y) \cdot(x ; g)
\end{aligned}
$$

for all $y \in \mathrm{Y}_{1}, x \in \mathrm{X}, g \in \mathrm{G}^{1}$ with $x=\hat{\mathrm{s}}_{\mathbf{Y}_{1}}(y)$ and $\mathrm{sX}_{\mathbf{X}}(x)=\mathrm{r}(g)$. Hence a map between two $X \rtimes \mathrm{G}$-actions is an $\mathrm{X} \rtimes \mathrm{G}$-map if and only if it is a $\mathrm{G}$-map and over $\mathrm{X}$.

The transformation groupoids $Y \rtimes(X \rtimes G)$ and $Y \rtimes G$ have the same objects $Y$. The arrows $\left(\mathrm{pr}_{1} ; \mathrm{pr}_{2} \circ \mathrm{pr}_{2}\right)$ and $\left(\mathrm{pr}_{1} ;\left(\hat{\mathrm{s}}_{\mathrm{Y}} \circ \mathrm{pr}_{1} ; \mathrm{pr}_{2}\right)\right)$ are maps between the arrows of these transformation groupoids, which are inverses of each other. These isomorphisms clearly intertwine the sorce, range and multiplication maps of these transformation groupoids. So they give a natural isomorphism between the groupoids $Y \rtimes(X \rtimes G)$ and $Y \rtimes G$.

5.3. Principal bundles. Let $\mathrm{G}=\left(\mathrm{G}^{0}, \mathrm{G}^{1}, \mathrm{r}, \mathrm{s}, \mathrm{m}, \mathrm{u}, \mathrm{i}\right)$ be a groupoid in a category $\left(\mathcal{C}, \mathcal{F}_{p}\right)$ with partial covers.

Definition 5.33. A right $\mathrm{G}$-action $\left(\mathrm{X} ; \mathrm{m}_{\mathrm{X}} ; \mathrm{s}_{\mathrm{X}}\right)$ is called a right $\mathrm{G}$-bundle over $\mathrm{p}: \mathbf{X} \rightarrow \mathbf{Z}$ if $\mathrm{p}: \mathbf{X} \rightarrow \mathbf{Z}$ is $\mathrm{G}$-invariant. A right $\mathrm{G}$-bundle over $\mathrm{p}: \mathbf{X} \rightarrow \mathbf{Z}$ is partially principal if 
(1) $\mathrm{p}$ is a partial cover;

(2) the arrow

$$
\left(\mathrm{pr}_{1} ; \mathrm{m}_{\mathrm{X}}\right): \mathrm{X} \times_{\mathrm{sX}_{\mathrm{X}}, \mathrm{G}_{0}, \mathrm{r}} \mathrm{G}^{1} \rightarrow \mathrm{X} \times_{\mathrm{p}, \mathrm{Z}, \mathrm{p}} \mathrm{X}, \quad(x ; g) \mapsto(x ; x \cdot g),
$$

is invertible.

A partially principal right G-bundle over $\mathrm{p}: \mathrm{X} \rightarrow \mathrm{Z}$ is called principal if $\mathrm{p}$ is a cover. Then we call $\mathbf{Z}$ and $\mathrm{p}$ an orbit space and orbit space projection of the G-action $\left(\mathrm{X} ; \mathrm{m}_{\mathrm{X}} ; \mathrm{s}_{\mathrm{X}}\right)$, respectively.

Lemma 5.34. An orbit space projection of a principal right $\mathrm{G}$-action $\left(\mathrm{X} ; \mathrm{m}_{\mathrm{X}} ; \mathrm{s}_{\mathrm{X}}\right)$ is a coequaliser of the arrows $\mathrm{pr}_{1}, \mathrm{~m}_{\mathrm{X}}: \mathrm{X} \times_{\mathrm{s}_{\mathrm{X}}, \mathrm{G}_{0}, \mathrm{r}} \mathrm{G}^{1} \rightrightarrows \mathrm{X}$.

Proof. Since (5.4) is an isomorphism, the equations $f \circ \mathrm{m}_{\mathrm{X}}=f \circ \mathrm{pr}_{1}$ and $f \circ \mathrm{pr}_{1}=f \circ \mathrm{pr}_{2}$ are equivalent. Since $\mathrm{p}$ is a cover, it is a coequaliser of $\mathrm{pr}_{1}, \mathrm{pr}_{2}: \mathrm{X} \times_{\mathrm{p}, \mathrm{Z}, \mathrm{p}} \mathrm{X} \rightrightarrows \mathrm{X}$. Therefore, it is a coequaliser of $\mathrm{pr}_{1}, \mathrm{~m}_{\mathrm{X}}: \mathrm{X} \times_{\mathrm{s}_{\mathrm{X}}, \mathrm{G}_{0}, \mathrm{r}} \mathrm{G}^{1} \rightrightarrows \mathrm{X}$.

Corollary 5.35. If a right $\mathrm{G}$-action $\left(\mathrm{X} ; \mathrm{m}_{\mathrm{X}} ; \mathrm{s}_{\mathrm{X}}\right)$ is a principal bundle, then the orbit space is unique up to isomorphism.

Proof. A coequaliser is unique up to isomorphism.

Remark 5.36. A left G-bundle is defined similarly. A left G-bundle is partially principal if $\mathrm{p}: \mathrm{X} \rightarrow \mathrm{Z}$ is a partial cover and the arrow

$$
\left(\mathrm{m}_{\mathrm{X}} ; \mathrm{pr}_{2}\right): \mathrm{G}^{1} \times_{\mathrm{s}_{\mathrm{G}}, \mathrm{G}_{0}, \mathrm{r}_{\mathrm{X}}} \mathrm{X} \stackrel{\sim}{\rightarrow} \mathrm{X} \times \times_{\mathrm{p}, \mathrm{Z}, \mathrm{p}} \mathrm{X}, \quad(g ; x) \mapsto(g \cdot x ; x),
$$

is invertible. If $\mathrm{p}$ is a cover then it is called principal.

Remark 5.37. A right $\mathrm{G}$-action is (partially) principal over $\mathrm{p}: \mathbf{X} \rightarrow \mathbf{Z}$ if and only if the corresponding left G-action described in Lemma 5.18 is (partially) principal over $\mathrm{p}: \mathrm{X} \rightarrow \mathrm{Z}$.

Remark 5.38. We denote the element $\mathrm{p}(x)$ in $\mathbf{Z}$ by $[x]$ for an element $x$ in $\mathbf{X}$. There are other elements in $\mathbf{Z}$ which are not given by the composition of $p$, but in the case of principal bundles it is enough to check some condition only for elements which are given by the composition with $\mathrm{p}$ because $\mathrm{p}$ is epic.

Definition 5.39. Let $\left(X ; m_{X} ; s_{X}\right)$ and $\left(\tilde{X} ; \tilde{m}_{X} ; \tilde{s}_{X}\right)$ be $G$-bundles over $p: X \rightarrow Z$ and $\tilde{\mathrm{p}}: \tilde{\mathrm{X}} \rightarrow \mathrm{Z}$, respectively. An arrow $f: \tilde{X} \rightarrow X$ is called a G-bundle map if it is a G-map and $\mathrm{p} \circ f=\tilde{\mathrm{p}}$. Elementwise $\mathrm{s}_{\mathbf{X}}(f(x))=\tilde{\mathbf{s}}_{\mathbf{X}}(x), f(x \cdot g)=f(x) \cdot g$ and $[f(x)]=[x]$ for all $x \in \tilde{\mathrm{X}}, g \in \mathrm{G}^{1}$ with $\tilde{\mathbf{s}}_{\mathbf{X}}(x)=\mathrm{r}(g)$.

\subsection{Examples of principal bundles.}

Example 5.40. Let $f: \mathrm{X} \rightarrow \mathrm{Y}$ be an arrow in $\left(\mathcal{C}, \mathcal{F}_{p}\right)$. The right $\mathrm{Y}$-action $\left(\mathrm{X} ; \mathrm{pr}_{1} ; f\right)$ described in Example 5.23 is a principal bundle over id $\mathrm{X}: \mathrm{X} \rightarrow \mathrm{X}$ because there is an obvious isomorphism $\left(\mathrm{pr}_{1} ; \mathrm{pr}_{1}\right): \mathrm{X} \times_{f, \mathrm{Y}, \mathrm{id} \mathrm{Y}} \mathrm{Y} \stackrel{\sim}{\rightarrow} \mathrm{X} \times_{\mathrm{idx}, \mathrm{X}, \mathrm{idx}} \mathrm{X}$.

Example 5.41. Let $\mathrm{p}: \mathrm{X} \rightarrow \mathrm{Z}$ be a partial cover and let $\mathrm{G}$ be the Čech groupoid of $\mathrm{p}$. The action of $\mathrm{G}$ on its objects $\left(\mathrm{X} ; \mathrm{pr}_{2} \circ \mathrm{pr}_{2} ; \mathrm{idx}\right.$ ) (see Example 5.21) is a partially principal bundle over $\mathrm{p}: X \rightarrow Z$. We just need to check that the arrow $\left(\mathrm{pr}_{1} ; \mathrm{pr}_{2} \circ \mathrm{pr}_{2}\right): \mathrm{X} \times_{\mathrm{id}_{\mathrm{X}}, \mathrm{X}, \mathrm{pr}_{1}}\left(\mathrm{X} \times_{\mathrm{p}, \mathrm{Z}, \mathrm{p}} \mathrm{X}\right) \rightarrow \mathrm{X} \times_{\mathrm{p}, \mathrm{Z}, \mathrm{p}} \mathrm{X}$ is an isomorphism. That is true because $\left(\mathrm{pr}_{1} ; \mathrm{pr}_{2} \circ \mathrm{pr}_{2}\right)=\left(\mathrm{pr}_{1} \circ \mathrm{pr}_{2} ; \mathrm{pr}_{2} \circ \mathrm{pr}_{2}\right)=\left(\mathrm{pr}_{1} ; \mathrm{pr}_{2}\right) \circ \mathrm{pr}_{2}=\mathrm{pr}_{2}$, and this coordinate projection is an isomorphism because it is a pull-back of idx.

Example 5.42. Let $\mathrm{G}=\left(\mathrm{G}^{0} ; \mathrm{G}^{1} ; \mathrm{r} ; \mathrm{s} ; \mathrm{m}\right)$ be a groupoid. The right translation action described in Example 5.24 is a principal G-bundle over $r: G^{1} \rightarrow G^{0}$. This follows from the well-defined isomorphism $(3.2)$. 
Example 5.43. The G-action $\left(\mathrm{L}^{1} ; \mathrm{m} ; \mathrm{r}\right)$ described in Example 5.27 is a partially principal bundle over $\left(\mathrm{F}^{1} ; \mathrm{s}_{\mathrm{L}}\right): \mathrm{L}^{1} \rightarrow \mathrm{H}^{1} \times_{\mathrm{S}_{\mathrm{H}}, \mathrm{H}^{0}, \mathrm{~F}^{0}} \mathrm{~L}^{0}$. We need to check that the arrow

$$
\mathrm{G}^{1} \times_{\mathrm{s}_{\mathrm{G}}, \mathrm{L}^{0}, \mathrm{r}_{\mathrm{L}}} \mathrm{L}^{1} \stackrel{\left(\mathrm{m} ; \mathrm{pr}_{2}\right)}{\longrightarrow} \mathrm{L}^{1} \times_{\left(\mathrm{F}^{1} ; \mathrm{sL}_{\mathrm{L}}\right),\left(\mathrm{H}^{1} \times_{\mathrm{S}_{\mathrm{H}}, \mathrm{H}^{0}, \mathrm{~F}^{0}} \mathrm{~L}^{0}\right),\left(\mathrm{F}^{1} ; \mathrm{S}_{\mathrm{L}}\right)} \mathrm{L}^{1}, \quad(g ; l) \mapsto(g \cdot l ; l),
$$

is well-defined and invertible. It is well-defined, and therefore, $\left(\mathrm{F}^{1} ; \mathrm{s}_{\mathrm{L}}\right)$ is $\mathrm{G}$-invariant, because $\mathrm{s}_{\mathrm{L}}(g \cdot l)=\mathrm{s}_{\mathrm{L}}(l)$ and

$$
\begin{aligned}
\mathrm{F}^{1}(g \cdot l) & =\mathrm{F}^{1}(g) \cdot \mathrm{F}^{1}(l) \\
& =\mathrm{F}^{1}\left(1_{\mathrm{s}_{\mathrm{L}}(g)}\right) \cdot \mathrm{F}^{1}(l) \\
& =1_{\mathrm{F}^{0}\left(\mathrm{r}_{\mathrm{L}}(l)\right)} \cdot \mathrm{F}^{1}(l) \\
& =1_{\mathrm{r}_{\mathrm{H}}\left(\mathrm{F}^{1}(l)\right)} \cdot \mathrm{F}^{1}(l) \\
& =\mathrm{F}^{1}(l)
\end{aligned}
$$

for all $g \in \mathrm{G}^{1}, l \in \mathrm{L}^{1}$ with $\mathrm{s}_{\mathrm{L}}(g)=\mathrm{r}_{\mathrm{L}}(l)$.

Consider the arrow

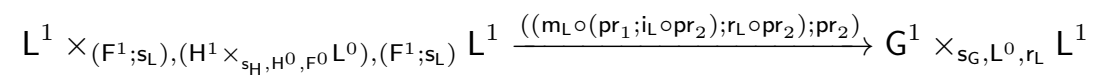

defined elementwise by $\left(l ; l_{1}\right) \mapsto\left(l \cdot l_{1}^{-1} ; l_{1}\right)$ for all $l, l_{1} \in \mathrm{L}^{1}$ with $\mathrm{F}^{1}(l)=\mathrm{F}^{1}\left(l_{1}\right)$ and $\mathrm{s}_{\mathrm{L}}(l)=\mathrm{s}_{\mathrm{L}}\left(l_{1}\right)$. It is well-defined because

$$
\begin{aligned}
\mathrm{F}^{1}\left(l \cdot l_{1}^{-1}\right) & =\mathrm{F}^{1}(l) \cdot \mathrm{F}^{1}\left(l_{1}^{-1}\right) \\
& =\mathrm{F}^{1}(l) \cdot\left(\mathrm{F}^{1}\left(l_{1}\right)\right)^{-1} \\
& =\mathrm{F}^{1}(l) \cdot\left(\mathrm{F}^{1}(l)\right)^{-1} \\
& =1_{\mathrm{rH}_{\mathrm{H}}\left(\mathrm{F}^{1}(l)\right)} \\
& =1_{\mathrm{r}_{\mathrm{H}}\left(\mathrm{F}^{1}\left(l_{1}\right)\right)} \\
& =1_{\mathrm{F}^{0}\left(\mathrm{r}_{\mathrm{L}}\left(l_{1}\right)\right)} \\
& =1_{\mathrm{F}^{0}\left(\mathrm{~s}_{\mathrm{L}}\left(l_{1}^{-1}\right)\right)} \\
& =1_{\mathrm{F}^{0}\left(\mathrm{~s}_{\mathrm{L}}\left(l \cdot l_{1}^{-1}\right)\right)} \\
& =\mathrm{F}^{1}\left(1_{\mathrm{s}_{\mathrm{L}}\left(l \cdot l_{1}^{-1}\right)}\right)
\end{aligned}
$$

and $\mathrm{s}_{\mathrm{L}}\left(l \cdot l_{1}^{-1}\right)=\mathrm{s}_{\mathrm{L}}\left(l_{1}^{-1}\right)=\mathrm{r}_{\mathrm{L}}\left(l_{1}\right)$ for all $l, l_{1} \in \mathrm{L}^{1}$ with $\mathrm{F}^{1}(l)=\mathrm{F}^{1}\left(l_{1}\right)$ and $\mathrm{s}_{\mathrm{L}}(l)=$ $\mathrm{s}_{\mathrm{L}}\left(l_{1}\right)$. We also have

$$
\begin{aligned}
\left(\mathrm{m} ; \mathrm{pr}_{2}\right)\left(\left(\left(\mathrm{m}_{\mathrm{L}} \circ\left(\mathrm{pr}_{1} ; \mathrm{i}_{\mathrm{L}} \circ \mathrm{pr}_{2}\right) ; \mathrm{r}_{\mathrm{L}} \circ \mathrm{pr}_{2}\right) ; \mathrm{pr}_{2}\right)\left(l ; l_{1}\right)\right) & =\left(\mathrm{m} ; \mathrm{pr}_{2}\right)\left(l \cdot l_{1}^{-1} ; l_{1}\right) \\
& =\left(\left(l \cdot l_{1}^{-1}\right) \cdot l_{1} ; l_{1}\right) \\
& =\left(l \cdot\left(l_{1}^{-1} \cdot l_{1}\right) ; l_{1}\right) \\
& =\left(l \cdot 1_{\mathrm{s}_{\mathrm{L}}\left(l_{1}\right)} ; l_{1}\right) \\
& =\left(l \cdot 1_{\mathrm{s}_{\mathrm{L}}(l)} ; l_{1}\right) \\
& =\left(l ; l_{1}\right)
\end{aligned}
$$


for all $l, l_{1} \in \mathrm{L}^{1}$ with $\mathrm{F}^{1}(l)=\mathrm{F}^{1}\left(l_{1}\right)$ and $\mathrm{s}_{\mathrm{L}}(l)=\mathrm{s}_{\mathrm{L}}\left(l_{1}\right)$. We also need to compute the composition in inverse order

$$
\begin{aligned}
& \left(\left(\mathrm{m}_{\mathrm{L}} \circ\left(\mathrm{pr}_{1} ; \mathrm{i}_{\mathrm{L}} \circ \mathrm{pr}_{2}\right) ; \mathrm{r}_{\mathrm{L}} \circ \mathrm{pr}_{2}\right) ; \mathrm{pr}_{2}\right)\left(\left(\mathrm{m} ; \mathrm{pr}_{2}\right)(g ; l)\right) \\
= & \left(\left(\mathrm{m}_{\mathrm{L}} \circ\left(\mathrm{pr}_{1} ; \mathrm{i}_{\mathrm{L}} \circ \mathrm{pr}_{2}\right) ; \mathrm{r}_{\mathrm{L}} \circ \mathrm{pr}_{2}\right) ; \mathrm{pr}_{2}\right)(g \cdot l ; l) \\
= & \left((g \cdot l) \cdot l^{-1} ; l\right) \\
= & \left(g \cdot\left(l \cdot l^{-1}\right) ; l\right) \\
= & \left(g \cdot 1_{\mathrm{r}_{\mathrm{L}}(l)} ; l\right) \\
= & \left(g \cdot 1_{\mathrm{s}_{\mathrm{L}}(g)} ; l\right) \\
= & (g ; l)
\end{aligned}
$$

for all $g \in \mathrm{G}^{1}, l \in \mathrm{L}^{1}$ with $\mathrm{s}_{\mathrm{L}}(g)=\mathrm{r}_{\mathrm{L}}(l)$. So $\left(\mathrm{m} ; \mathrm{pr}_{2}\right)$ is invertible. Therefore, the G-action $\left(\mathrm{L}^{1} ; \mathrm{m} ; \mathrm{r}\right)$ described in Example 5.27 is a partially principal bundle over

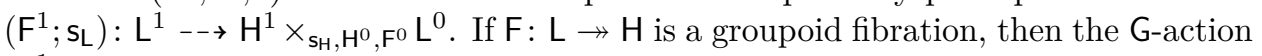
$\left(\mathrm{L}^{1} ; \mathrm{m} ; \mathrm{r}\right)$ is a principal bundle.

5.5. Pull-back of a bundle. Let $\mathrm{G}=\left(\mathrm{G}^{0} ; \mathrm{G}^{1} ; \mathrm{r} ; \mathrm{s} ; \mathrm{m}\right)$ be a groupoid in the category $\left(\mathcal{C}, \mathcal{F}_{p}\right)$ with partial covers.

Proposition 5.44. Let $\left(\mathrm{Y} ; \mathrm{m}_{\mathrm{Y}} ; \mathrm{s}_{\mathrm{Y}}\right)$ be a $\mathrm{G}$-bundle over $\mathrm{p}_{\mathrm{Y}}: \mathrm{Y} \rightarrow \mathrm{Z}_{\mathrm{Y}}$. If the diagram

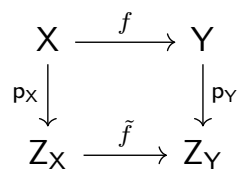

is a pull-back square, then there is a $\mathrm{G}$-bundle $\left(\mathrm{X} ; \mathrm{m}_{\mathbf{X}} ; \mathrm{s}_{\mathbf{X}}\right)$ over $\mathrm{p}_{\mathbf{X}}: \mathrm{X} \rightarrow \mathrm{Z}_{\mathbf{X}}$, such that $f: \mathrm{X} \rightarrow \mathrm{Y}$ is a $\mathrm{G}-m a p$, and this construction is unique.

This G-bundle is called the pull-back of the G-bundle (Y; $\mathrm{m}_{Y} ; \mathrm{s}_{\mathrm{Y}}$ ) along the arrow $\tilde{f}: \mathbf{Z}_{\mathbf{X}} \rightarrow \mathbf{Z}_{\mathbf{Y}}$.

Proof. Firstly, it is clear that the anchor map sx has to be equal to syof. We also need the following equations to hold: $\mathrm{p}_{\mathrm{X}} \circ \mathrm{m}_{\mathrm{X}}=\mathrm{p}_{\mathrm{X}} \circ \mathrm{pr}_{1}$ and $f \circ \mathrm{m}_{\mathrm{X}}=\mathrm{m}_{\mathrm{Y}} \circ\left(f \circ \mathrm{pr}_{1} ; \mathrm{pr}_{2}\right)$. This determines $\mathrm{m}_{\mathrm{X}}$ uniquely. Therefore, $\mathrm{m}_{\mathrm{X}}=\left(\mathrm{p}_{\mathrm{X}} \circ \mathrm{pr}_{1} ; \mathrm{m}_{\mathrm{Y}} \circ\left(f \circ \mathrm{pr}_{1} ; \mathrm{pr}_{2}\right)\right)$. Elementwise, $(z ; y) \cdot g=(z ; y \cdot g)$ for all $z \in \mathrm{Z}_{\mathbf{X}}, y \in \mathrm{Y}, g \in \mathrm{G}^{1}$ with $\tilde{f}(z)=\operatorname{pr}_{\mathbf{Y}}(y)$ and $\mathrm{s}_{\mathbf{Y}}(y)=\mathrm{r}(g)$. The map $\mathrm{m}_{\mathrm{X}}$ is well-defined because $\tilde{f}(z)=\mathrm{p}_{\mathbf{Y}}(y)=\mathrm{p}_{\mathbf{Y}}(y \cdot g)$.

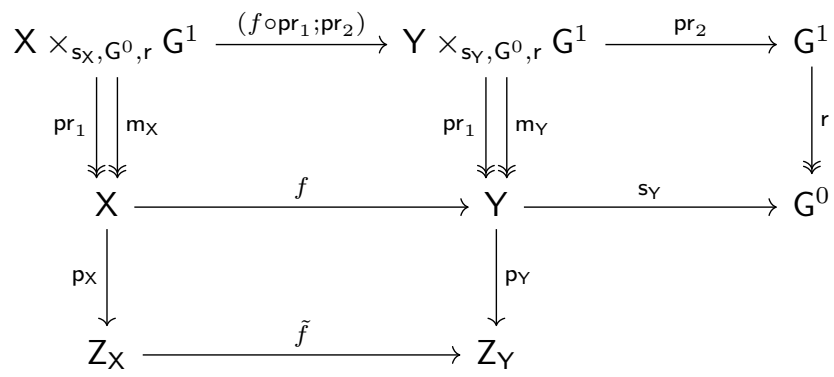

We have to show that $\left(\mathrm{X} ; \mathrm{m}_{\mathbf{X}} ; \mathrm{sX}_{\mathbf{X}}\right)$ is a $\mathrm{G}$-action. We have

$$
\begin{aligned}
\mathrm{sX}_{\mathrm{X}}((z ; y) \cdot g) & =\mathrm{sX}(z ; y \cdot g) \\
& =\mathrm{s}_{\mathbf{Y}}(y \cdot g) \\
& =\mathrm{s}(g)
\end{aligned}
$$


for all $z \in \mathrm{Z}_{\mathbf{X}}, y \in \mathrm{Y}, g \in \mathrm{G}^{1}$ with $\tilde{f}(z)=\mathrm{p}_{\mathbf{Y}}(y)$ and $\mathrm{s}_{\mathbf{Y}}(y)=\mathrm{r}(g)$. Property (1) in Definition 5.1 is shown. $\mathrm{m}_{\mathrm{X}}$ commutes with $\mathrm{m}$ because

$$
\begin{aligned}
((z ; y) \cdot g) \cdot g_{1} & =(z ; y \cdot g) \cdot g_{1} \\
& =\left(z ;(y \cdot g) \cdot g_{1}\right) \\
& =\left(z ; y \cdot\left(g \cdot g_{1}\right)\right) \\
& =(z ; y) \cdot\left(g \cdot g_{1}\right)
\end{aligned}
$$

for all $z \in \mathbf{Z}_{\mathbf{X}}, y \in \mathbf{Y}, g, g_{1} \in \mathrm{G}^{1}$ with $\tilde{f}(z)=\mathrm{p}_{\mathbf{Y}}(y), \mathrm{s}_{\mathbf{Y}}(y)=\mathrm{r}(g)$ and $\mathrm{s}(g)=\mathrm{r}\left(g_{1}\right)$. We also have

$$
\begin{aligned}
(z ; y) \cdot 1_{\mathrm{sX}(z ; y)} & =\left(z ; y \cdot 1_{\mathrm{sX}_{(}(z ; y)}\right) \\
& =\left(z ; y \cdot 1_{\mathrm{sY}_{(}(y)}\right) \\
& =(z ; y)
\end{aligned}
$$

for all $z \in \mathrm{Z}_{\mathbf{X}}, y \in \mathrm{Y}$, with $\tilde{f}(z)=\mathrm{p}_{\mathbf{Y}}(y)$. So Lemma 5.7 works. Hence $\left(\mathrm{X} ; \mathrm{m}_{\mathbf{X}} ; \mathbf{s}_{\mathbf{X}}\right)$ is a G-action, and therefore, it is a G-bundle over $p_{X}: X \rightarrow Z_{X}$.

Remark 5.45. The construction in Proposition 5.44 is for a right G-action. For a left action the construction is similar. The only change is the action map. The pull back of a left $G$-bundle $\left(Y ; m_{Y} ; r_{Y}\right)$ over $p_{Y}: Y \rightarrow Z_{Y}$ along the arrow $\tilde{f}: Z_{X} \rightarrow Z_{Y}$ is a G-bundle $\left(\mathrm{X} ;\left(\mathrm{p}_{\mathrm{X}} \circ \mathrm{pr}_{2} ; \mathrm{m}_{Y} \circ\left(\mathrm{pr}_{1} ; f \circ \mathrm{pr}_{2}\right)\right) ; \mathrm{r}_{\mathbf{Y}} \circ f\right)$ over $\mathrm{p}_{\mathrm{X}}: \mathrm{X} \rightarrow Z_{\mathrm{X}}$. The action map is defined elementwise by $g \cdot(z ; y)=(z ; g \cdot y)$ for all $z \in \mathrm{Z}_{\mathrm{X}}, y \in \mathrm{Y}, g \in \mathrm{G}^{1}$ with $\tilde{f}(z)=\operatorname{pr}_{\mathbf{Y}}(y)$ and $\mathrm{r}_{\mathbf{Y}}(y)=\mathrm{s}(g)$.

Proposition 5.46. Let $\left(\mathrm{X} ; \mathrm{m}_{\mathbf{X}} ; \mathrm{s}_{\mathrm{X}}\right)$ be a partially principal $\mathrm{G}$-bundle over $\mathrm{p}: \mathrm{X} \rightarrow \mathrm{Z}$. Let $f: \tilde{Z} \rightarrow Z$ be any arrow. There are a partially principal $\mathrm{G}$-bundle $\left(\tilde{\mathbf{X}} ; \tilde{m}_{\mathbf{X}} ; \tilde{s}_{\mathbf{X}}\right)$ over $\tilde{\mathrm{p}}: \tilde{\mathrm{X}} \rightarrow \tilde{\mathrm{Z}}$ and $a \mathrm{G}-$ map $\alpha: \tilde{\mathrm{X}} \rightarrow \mathrm{X}$ with $\mathrm{p} \circ \alpha=f \circ \tilde{\mathrm{p}}$.

This partially principal G-bundle is called the pull-back of the partially principal G-bundle $\left(\mathrm{X} ; \mathrm{m}_{\mathbf{X}} ; \mathrm{sX}\right)$ over $\mathrm{p}: \mathrm{X} \rightarrow \mathrm{Z}$ along the arrow $f: \tilde{Z} \rightarrow \mathrm{Z}$.

Proof. Let $\tilde{\mathrm{X}}=\tilde{\mathrm{Z}} \times_{f, Z, \mathrm{p}} \mathrm{X}, \tilde{\mathrm{p}}=\mathrm{pr}_{1}$ and $\alpha=\mathrm{pr}_{2}$. We know that this fibre product exists and $\tilde{p}=\mathrm{pr}_{1}$ is a partial cover because $p: X \rightarrow Z$ is. Now we can use Proposition 5.44 Consider the pull-back of the G-bundle $\left(\mathrm{X} ; \mathrm{m}_{\mathrm{X}} ; \mathrm{s}_{\mathrm{X}}\right)$ over $\mathrm{p}: \mathrm{X} \rightarrow \mathrm{Z}$ along the arrow $f: \tilde{Z} \rightarrow Z$. That is $\left(\tilde{Z} \times_{f, Z, p} X ;\left(p_{1} \circ p_{1} ; \mathrm{m}_{\mathbf{X}} \circ\left(\mathrm{pr}_{2} \circ \mathrm{pr}_{1} ; \mathrm{pr}_{2}\right)\right) ; s_{\mathbf{X}} \circ \mathrm{pr}_{2}\right)$ over $\mathrm{pr}_{1}: \tilde{Z} \times_{f, Z, \mathrm{p}} \mathrm{X} \rightarrow \tilde{Z}$. This action is defined elementwise by $(z ; x) \cdot g=(z ; x \cdot g)$ for all $z \in \tilde{Z}, x \in \mathrm{X}, g \in \mathrm{G}^{1}$ with $f(z)=\mathrm{p}(y)$ and $\mathrm{s}_{\mathbf{X}}(x)=\mathrm{r}(g)$. We have to prove that it is partially principal. We need to check that $\left(\mathrm{pr}_{1} ; \tilde{\mathrm{m}}_{X}\right)$ is invertible, where $\tilde{\mathrm{m}}_{\mathrm{X}}=\left(\mathrm{pr}_{1} \circ \mathrm{pr}_{1} ; \mathrm{m}_{\mathrm{X}} \circ\left(\mathrm{pr}_{2} \circ \mathrm{pr}_{1} ; \mathrm{pr}_{2}\right)\right)$. Let us show that the following arrow

$$
\left(\tilde{\mathrm{Z}} \times_{f, Z, \mathrm{p}} \mathrm{X}\right) \times_{\mathrm{sx}_{\mathrm{X}} \circ \mathrm{or}_{2}, \mathrm{G}^{0}, \mathrm{r}} \mathrm{G}^{1} \stackrel{\left(\mathrm{pr}_{1} ; \tilde{\mathrm{m}}_{\mathrm{X}}\right)}{\longrightarrow}\left(\tilde{\mathrm{Z}} \times_{f, \mathrm{Z}, \mathrm{p}} \mathrm{X}\right) \times_{\mathrm{pr}_{1}, \tilde{Z}, \mathrm{pr}_{1}}\left(\tilde{\mathrm{Z}} \times_{f, Z, \mathrm{p}} \mathrm{X}\right)
$$

is an inverse of $\left(\mathrm{pr}_{1} ; \tilde{\mathrm{m}}_{\mathrm{X}}\right)$. It is defined elementwise by $\left((z ; x) ;\left(z ; x_{1}\right)\right) \mapsto((z ; x) ; g)$, for all $z \in \tilde{\mathbf{Z}}, x, x_{1} \in \mathrm{X}$ with $\mathrm{p}(x)=f(z)=\mathrm{p}\left(x_{1}\right)$, where the element $g \in \mathrm{G}^{1}$ is the unique one with $g \cdot g=x_{1}$. This arrow is well-defined because

$$
\begin{aligned}
\mathrm{sX}_{\mathbf{X}}(x) & =\mathrm{sx}_{\mathbf{X}}\left(x_{1} \cdot g^{-1}\right) \\
& =\mathrm{s}\left(g^{-1}\right) \\
& =\mathrm{r}(g) .
\end{aligned}
$$


Consider the composition:

$$
\begin{aligned}
& \left(\mathrm{pr}_{1} ; \mathrm{pr}_{2} \circ\left(\mathrm{pr}_{1} ; \mathrm{m}_{\mathrm{X}}\right)^{-1} \circ\left(\mathrm{pr}_{2} \circ \mathrm{pr}_{1} ; \mathrm{pr}_{2} \circ \mathrm{pr}_{2}\right)\right)\left(\left(\mathrm{pr}_{1} ; \tilde{\mathrm{m}}_{\mathrm{X}}\right)((z ; x) ; g)\right) \\
= & \left(\mathrm{pr}_{1} ; \mathrm{pr}_{2} \circ\left(\mathrm{pr}_{1} ; \mathrm{m}_{\mathrm{X}}\right)^{-1} \circ\left(\mathrm{pr}_{2} \circ \mathrm{pr}_{1} ; \mathrm{pr}_{2} \circ \mathrm{pr}_{2}\right)\right)((z ; x) ;(z ; x) \cdot g) \\
= & \left(\mathrm{pr}_{1} ; \mathrm{pr}_{2} \circ\left(\mathrm{pr}_{1} ; \mathrm{m}_{\mathrm{X}}\right)^{-1} \circ\left(\mathrm{pr}_{2} \circ \mathrm{pr}_{1} ; \mathrm{pr}_{2} \circ \mathrm{pr}_{2}\right)\right)((z ; x) ;(z ; x \cdot g)) \\
= & ((z ; x) ; g)
\end{aligned}
$$

for all $z \in \tilde{\mathrm{Z}}, x \in \mathrm{X}, g \in \mathrm{G}^{1}$ with $\mathrm{p}(x)=f(z)$ and $\mathrm{s}_{\mathbf{X}}(x)=\mathrm{r}(g)$. Also,

$$
\begin{aligned}
& \left(\mathrm{pr}_{1} ; \tilde{\mathrm{m}}_{\mathrm{X}}\right)\left(\left(\mathrm{pr}_{1} ; \mathrm{pr}_{2} \circ\left(\mathrm{pr}_{1} ; \mathrm{m}_{\mathbf{X}}\right)^{-1} \circ\left(\mathrm{pr}_{2} \circ \mathrm{pr}_{1} ; \mathrm{pr}_{2} \circ \mathrm{pr}_{2}\right)\right)\left((z ; x) ;\left(z ; x_{1}\right)\right)\right) \\
= & \left(\mathrm{pr}_{1} ; \tilde{\mathrm{m}}_{\mathrm{X}}\right)((z ; x) ; g) \\
= & ((z ; x) ;(z ; x) \cdot g) \\
= & ((z ; x) ;(z ; x \cdot g)) \\
= & \left((z ; x) ;\left(z ; x_{1}\right)\right)
\end{aligned}
$$

for all $z \in \tilde{Z}, x, x_{1} \in \mathrm{X}$ with $\mathrm{p}(x)=f(z)=\mathrm{p}\left(x_{1}\right)$. So $\left(\mathrm{pr}_{1} ; \tilde{\mathrm{m}}_{\mathbf{X}}\right)$ is an isomorphism and therefore, $\left(\tilde{Z} \times_{f, Z, p} X ;\left(\mathrm{pr}_{1} \circ \mathrm{pr}_{1} ; \mathrm{m}_{\mathrm{X}} \circ\left(\mathrm{pr}_{2} \circ \mathrm{pr}_{1} ; \mathrm{pr}_{2}\right)\right) ; \mathrm{sX}_{\mathrm{X}} \circ \mathrm{pr}_{2}\right)$ is a partially principal G-bundle over $\operatorname{pr}_{1}: \tilde{Z} \times_{f, Z, p} X \rightarrow \tilde{Z}$.

Remark 5.47. Under Assumption 2.15 we have the same result as Proposition 5.46 in the global case. Let $\left(\mathbf{X} ; \mathrm{m}_{\mathbf{X}} ; \mathrm{s}_{\mathbf{X}}\right)$ be a principal G-bundle over $\mathrm{p}: \mathbf{X} \rightarrow \mathbf{Z}$. Let $f: \mathbf{Z} \rightarrow \mathbf{Z}$ be any arrow. There are a principal G-bundle $\left(\tilde{X} ; \tilde{m}_{X} ; \tilde{s}_{X}\right)$ over $\tilde{p}: \tilde{X} \rightarrow \tilde{Z}$ and a G-map $\alpha: \tilde{X} \rightarrow \mathrm{X}$ with $\mathrm{p} \circ \alpha=f \circ \tilde{\mathrm{p}}$. The construction is absolutely the same. The only difference is the conclusion that the coordinate projection $\operatorname{pr}_{1}: \tilde{Z} \times_{f, Z, p} X \rightarrow \tilde{Z}$ is a cover instead of a partial cover by Assumption 2.15

Proposition 5.48. Assume Assumption 2.15. Let $\left(\mathrm{X} ; \mathrm{m}_{\mathrm{X}} ; \mathrm{s}_{\mathrm{X}}\right)$ be a partially principal G-bundle over $\mathrm{p}: \mathrm{X} \rightarrow \mathrm{Z}$. Let $f: \tilde{\mathrm{Z}} \rightarrow \mathbf{Z}$ be any arrow. Let $\left(\hat{\mathbf{X}} ; \hat{\mathrm{m}}_{\mathbf{X}} ; \hat{\mathrm{s}}_{\mathbf{X}}\right)$ be a principal $\mathrm{G}$-bundle over $\hat{\mathrm{p}}: \hat{\mathrm{X}} \rightarrow \tilde{\mathbf{Z}}$ with a $\mathrm{G}-$ map $\alpha: \hat{\mathrm{X}} \rightarrow \mathrm{X}$ with $\mathrm{p} \circ \alpha=f \circ \hat{\mathrm{p}}$. Then this principal G-bundle is bundle isomorphic to the pull-back of the partially principal $\mathrm{G}$-bundle $\left(\mathrm{X} ; \mathrm{m}_{\mathbf{X}} ; \mathrm{s}_{\mathbf{X}}\right)$ over $\mathrm{p}: \mathbf{X} \rightarrow \mathbf{Z}$ along the arrow $f: \tilde{\mathbf{Z}} \rightarrow \mathbf{Z}$ (described in Proposition 5.46), which automatically is a principal G-bundle.

Proof. Our data is depicted in the following diagram:

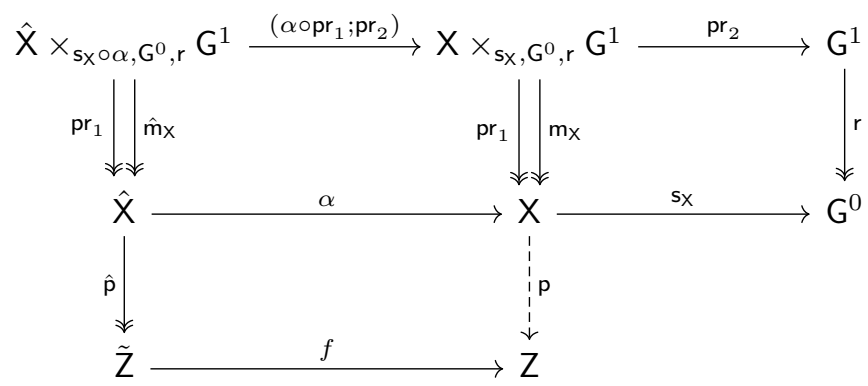

There is a well-defined arrow $(\hat{\mathbf{p}} ; \alpha): \hat{\mathbf{X}} \rightarrow \tilde{\mathbf{Z}} \times_{f, \mathrm{Z}, \mathrm{p}} \mathrm{X}$. It is a G-bundle map because $\operatorname{pr}_{1} \circ(\hat{\mathrm{p}} ; \alpha)=\hat{\mathrm{p}}$ and $(\hat{\mathrm{p}} ; \alpha) \circ \hat{\mathrm{m}}_{\mathbf{X}}=\tilde{\mathrm{m}}_{\mathrm{X}} \circ\left((\hat{\mathrm{p}} ; \alpha) \circ \mathrm{pr}_{1} ; \mathrm{pr}_{2}\right)$, where $\tilde{\mathrm{m}}_{\mathrm{X}}$ is the action map of the pull-back of the partially principal G-bundle $\left(X ; m_{X} ; s_{X}\right)$ over $p: X-\rightarrow Z$ along $f: \tilde{Z} \rightarrow Z$. The previous equation holds because

$$
\begin{aligned}
(\hat{\mathrm{p}} ; \alpha)(x \cdot g) & =(\hat{\mathrm{p}}(x \cdot g) ; \alpha(x \cdot g)) \\
& =(\hat{\mathrm{p}}(x) ; \alpha(x) \cdot g) \\
& =(\hat{\mathrm{p}}(x) ; \alpha(x)) \cdot g \\
& =(\hat{\mathrm{p}} ; \alpha)(x) \cdot g
\end{aligned}
$$


for all $x \in \hat{\mathrm{X}}, g \in \mathrm{G}^{1}$ with $\hat{\mathrm{s}}_{\mathbf{X}}(x)=\mathrm{r}(g)$. So $(\hat{\mathrm{p}} ; \alpha)$ is a G-bundle map. We are going to show that $(\hat{\mathrm{p}} ; \alpha)$ is an isomorphism. Consider the following diagram:

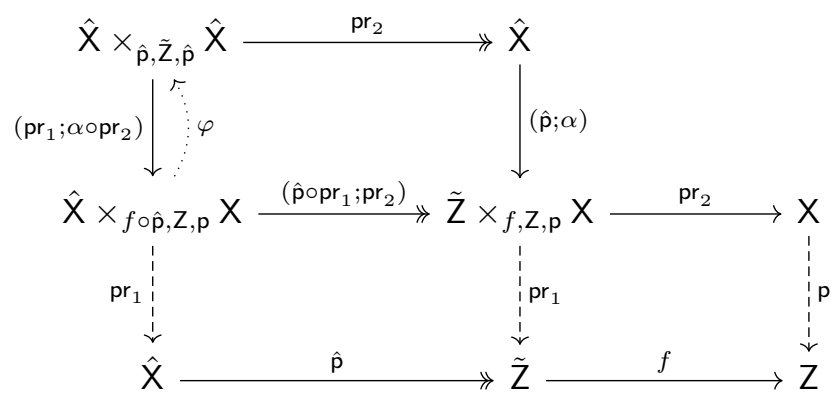

It is clear that all squares are pull-back squares by the well-known lemma about a rectangle of a pull-back squares. The arrow

$$
\left(\hat{\mathrm{p}} \circ \mathrm{pr}_{1} ; \mathrm{pr}_{2}\right): \hat{\mathrm{X}} \times_{f \circ \hat{\mathrm{p}}, \mathrm{Z}, \mathrm{p}} \mathrm{X} \rightarrow \tilde{\mathrm{Z}} \times_{f, \mathrm{Z}, \mathrm{p}} \mathrm{X}
$$

is a cover because of Assumption 2.15. Let us show that the arrow

$$
\left(\mathrm{pr}_{1} ; \alpha \circ \mathrm{pr}_{2}\right): \hat{\mathrm{X}} \times_{\hat{\mathrm{p}}, \tilde{\mathrm{Z}}, \hat{\mathrm{p}}} \hat{\mathrm{X}} \rightarrow \hat{\mathrm{X}} \times_{f \circ \hat{\mathrm{p}}, \mathrm{Z}, \mathrm{p}} \mathrm{X}
$$

has an inverse $\varphi$. Let $\varphi=\left(\operatorname{pr}_{1} ; \hat{m}_{\mathbf{X}}\right) \circ\left(\operatorname{pr}_{1} ; \psi\right)$, where $\psi$ is the composition

$$
\hat{\mathrm{X}} \times_{f \circ \hat{p}, Z, \mathrm{p}} \mathrm{X} \stackrel{\left(\alpha \circ \mathrm{pr}_{1} ; \mathrm{pr}_{2}\right)}{\longrightarrow} \mathrm{X} \times_{\mathrm{p}, \mathrm{Z}, \mathrm{p}} \mathrm{X} \stackrel{\left(\mathrm{pr}_{1} ; \mathrm{m}_{\mathrm{X}}\right)^{-1}}{\longrightarrow} \mathrm{X} \times_{\mathrm{sX}_{\mathrm{X}}, \mathrm{G}^{0}, \mathrm{r}} \mathrm{G}^{1} \stackrel{\mathrm{pr}_{2}}{\longrightarrow} \mathrm{G}^{1},
$$

defined elementwise by $\psi\left(x ; x_{1}\right)=g$ for all $x \in \hat{\mathrm{X}}, x_{1} \in \mathrm{X}$ with $f(\hat{\mathrm{p}}(x))=\mathrm{p}\left(x_{1}\right)$, where $g$ is the unique element in $\mathrm{G}^{1}$ such that $\alpha(x) \cdot g=x_{1}$. So $\varphi$ is defined elementwise by $\varphi\left(x ; x_{1}\right)=(x ; x \cdot g)$ for all $x \in \hat{\mathbf{X}}, x_{1} \in \mathbf{X}$ with $f(\hat{\mathrm{p}}(x))=\mathrm{p}\left(x_{1}\right)$, where $g$ is the unique element in $\mathrm{G}^{1}$ such that $\alpha(x) \cdot g=x_{1}$. Here the arrow $\left(\alpha \circ \mathrm{pr}_{1} ; \mathrm{pr}_{2}\right)$ is well-defined because $\mathrm{p} \circ \alpha \circ \mathrm{pr}_{1}=f \circ \hat{\mathrm{p}} \circ \mathrm{pr}_{1}=\mathrm{p} \circ \mathrm{pr}_{2}$. The arrow

$$
\left(\mathrm{pr}_{1} ; \psi\right): \hat{\mathrm{X}} \times_{f \circ \hat{\mathrm{p}}, \mathrm{Z}, \mathrm{p}} \mathrm{X} \rightarrow \hat{\mathrm{X}} \times_{\mathrm{sx} \circ \alpha, \mathrm{G}^{0}, \mathrm{r}} \mathrm{G}^{1}
$$

is well-defined because $\mathbf{s}_{\mathbf{X}}(\alpha(x))=\mathbf{s}_{\mathbf{X}}\left(x_{1} \cdot g^{-1}\right)=\mathbf{s}\left(g^{-1}\right)=\mathrm{r}(g)$ for all $x, x_{1} \in \mathbf{X}$ with $f(\hat{\mathrm{p}}(x))=\mathrm{p}\left(x_{1}\right)$.

We compute

$$
\begin{aligned}
\left(\mathrm{pr}_{1} ; \alpha \circ \mathrm{pr}_{2}\right)\left(\varphi\left(x ; x_{1}\right)\right) & =\left(\mathrm{pr}_{1} ; \alpha \circ \mathrm{pr}_{2}\right)(x ; x \cdot g) \\
& =(x ; \alpha(x \cdot g)) \\
& =(x ; \alpha(x) \cdot g) \\
& =\left(x ; x_{1}\right)
\end{aligned}
$$

for all $x, x_{1} \in \mathbf{X}$ with $f(\hat{\mathrm{p}}(x))=\mathrm{p}\left(x_{1}\right)$ and

$$
\begin{aligned}
\varphi\left(\left(\mathrm{pr}_{1} ; \alpha \circ \mathrm{pr}_{2}\right)\left(x ; x_{1}\right)\right) & =\varphi\left(x ; \alpha\left(x_{1}\right)\right) \\
& =(x ; x \cdot g)
\end{aligned}
$$

for all $x, x_{1} \in \mathrm{X}$ with $\hat{\mathrm{p}}(x)=\hat{\mathrm{p}}\left(x_{1}\right)$, where $g$ is the unique element in $\mathrm{G}^{1}$ such that $\alpha(x) \cdot g=\alpha\left(x_{1}\right)$ for all $x, x_{1} \in \mathbf{X}$ with $\hat{\mathrm{p}}(x)=\hat{\mathrm{p}}\left(x_{1}\right)$. Since $\hat{\mathrm{p}}(x)=\hat{\mathrm{p}}\left(x_{1}\right)$, there is the unique $g_{1} \in \mathrm{G}^{1}$ such that $x \cdot g_{1}=x_{1}$. Thus $\alpha\left(x_{1}\right)=\alpha\left(x \cdot g_{1}\right)=\alpha(x) \cdot g_{1}$. Therefore, $g=g_{1}$. Thus $\varphi\left(\left(\mathrm{pr}_{1} ; \alpha \circ \mathrm{pr}_{2}\right)\left(x ; x_{1}\right)\right)=(x ; x \cdot g)=\left(x ; x \cdot g_{1}\right)=\left(x ; x_{1}\right)$ for all $x, x_{1} \in \mathrm{X}$ with $\hat{\mathrm{p}}(x)=\hat{\mathrm{p}}\left(x_{1}\right)$. So $\left(\mathrm{pr}_{1} ; \alpha \circ \mathrm{pr}_{2}\right)$ is an isomorphism. Since it is the pull-back of $(\hat{\mathrm{p}} ; \alpha)$ along the cover $\left(\hat{\mathrm{p}} \circ \mathrm{pr}_{1} ; \mathrm{pr}_{2}\right),(\hat{\mathrm{p}} ; \alpha)$ is an isomorphism by Lemma 2.13 Thus the principal G-bundle $\left(\hat{X} ; \hat{m}_{X} ; \hat{s} X\right)$ over $\hat{p}: \hat{X} \rightarrow \tilde{Z}$ is isomorphic to the pull-back of the partially principal G-bundle $\left(X ; m_{X} ; s_{X}\right)$ over $p: X-\rightarrow Z$ along the arrow $f: \tilde{Z} \rightarrow Z$. Hence this pull-back is a principal bundle, too. Thus the coordinate projection $\mathrm{pr}_{1}: \tilde{Z} \times_{f, Z, \mathrm{p}} \mathrm{X} \rightarrow \tilde{\mathrm{Z}}$ is a cover. 
Corollary 5.49. Assume Assumption 2.15. Let $\left(\mathrm{X} ; \mathrm{m}_{\mathrm{X}} ; \mathrm{s}_{\mathrm{X}}\right)$ be a principal $\mathrm{G}-$ bundle over $\mathrm{p}: \mathrm{X} \rightarrow \mathrm{Z}$. Then its pull-back along any $f: \tilde{\mathrm{Z}} \rightarrow \mathbf{Z}$ is a principal bundle, and it is unique in the following sense: for any principal $\mathrm{G}-$ bundle $\left(\hat{\mathrm{X}} ; \hat{\mathrm{m}}_{\mathbf{X}} ; \hat{\mathrm{s}}_{\mathbf{X}}\right)$ over $\hat{\mathrm{p}}: \hat{\mathrm{X}} \rightarrow \mathbf{Z}$ and for any $\mathrm{G}-$ map $g: \hat{\mathrm{X}} \rightarrow \mathbf{X}$ with $\mathrm{p} \circ g=f \circ \hat{\mathrm{p}}$, this principal G-bundle is bundle isomorphic to the pull-back of $\left(\mathrm{X} ; \mathrm{m}_{\mathbf{X}} ; \mathrm{s}_{\mathbf{X}}\right)$ along $f: \tilde{\mathrm{Z}} \rightarrow \mathbf{Z}$.

Proof. The coordinate projection $\mathrm{pr}_{1}: \tilde{Z} \times_{f, \mathrm{Z}, \mathrm{p}} \mathrm{X} \rightarrow \tilde{\mathrm{Z}}$ is a cover by Assumption 2.15 because $\mathrm{p}: \mathrm{X} \rightarrow \mathrm{Z}$ is a cover. The other things are the same as in the proof of Proposition 5.48

Lemma 5.50. Let $\left(\mathrm{X} ; \mathrm{m}_{\mathrm{X}} ; \mathrm{s}_{\mathrm{X}}\right)$ be the pull-back of a $\mathrm{G}$-bundle $\left(\mathrm{Y} ; \mathrm{m}_{\mathrm{Y}} ; \mathrm{s}_{\mathrm{Y}}\right)$ over $\beta: \mathrm{Y} \rightarrow \mathrm{A}$ along a partial cover $f: \mathrm{A} \rightarrow \mathrm{B}$ (Proposition 5.44). If $\left(\mathrm{Y} ; \mathrm{m}_{\mathrm{Y}} ; \mathrm{s}_{\mathrm{Y}}\right)$ is a principal $\mathrm{G}$-bundle over $\mathrm{p}_{\mathrm{Y}}: \mathrm{Y} \rightarrow \mathrm{Z}$, then $\left(\mathrm{X} ; \mathrm{m}_{\mathrm{X}} ; \mathrm{s}_{\mathrm{X}}\right)$ is a partially principal $\mathrm{G}$ bundle over $\left(\mathrm{pr}_{1} ; \mathrm{p}_{\mathrm{Y}} \circ \mathrm{pr}_{2}\right): \mathrm{X} \rightarrow \mathrm{B} \times_{f, \mathrm{~A}, \alpha} \mathrm{Z}$, where $\alpha: \mathrm{Z} \rightarrow \mathrm{A}$ is a unique factorization of $\mathrm{p}_{\mathrm{Y}}: \mathrm{Y} \rightarrow \mathrm{Z}$ by $\beta: \mathrm{Y} \rightarrow \mathrm{A}$. Under Assumption 2.15. $\left(\mathrm{X} ; \mathrm{m}_{\mathbf{X}} ; \mathrm{s}_{\mathbf{X}}\right)$ is a principal bundle, too.

Proof. The arrow $\alpha: Z \rightarrow A$ exists because $\mathrm{p}_{Y}: \mathrm{Y} \rightarrow \mathrm{Z}$ is a coequaliser of the parallel arrows $\mathrm{pr}_{1}, \mathrm{~m}_{\mathrm{Y}}: \mathrm{Y} \times_{\mathrm{sY}_{\mathrm{Y}}, \mathrm{G}^{0}, \mathrm{r}} \mathrm{G}^{1} \rightrightarrows \mathrm{Y}$ and $\beta \circ \mathrm{pr}_{1}=\beta \circ \mathrm{m}_{\mathrm{Y}}$. For any element $y$ in $\mathrm{Y}$ we have $\alpha([y])=\beta(y)$.

We have the following diagram

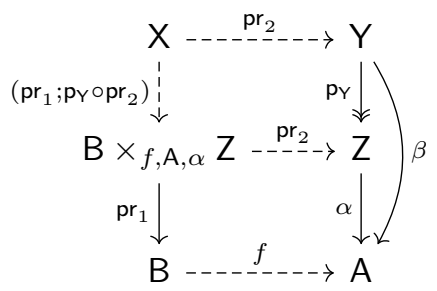

of pull-back squares, which shows that the upper square is a pull-back square, too. Therefore, the arrow $\left(\mathrm{pr}_{1} ; \mathrm{pY}_{\mathrm{Y}} \circ \mathrm{pr}_{2}\right): \mathrm{X} \rightarrow \mathrm{B} \times_{f, \mathrm{~A}, \alpha} \mathrm{Z}$ is a partial cover because it is the pull-back of the cover $p_{Y}: Y \rightarrow Z$. Because of Propositions 5.46 and 5.44 the G-bundle $\left(X ; m_{X} ; s_{X}\right)$ over $\left(\mathrm{pr}_{1} ; \mathrm{p}_{\mathbf{Y}} \circ \mathrm{pr}_{2}\right): \mathrm{X} \rightarrow \mathrm{B} \times_{f, \mathrm{~A}, \alpha} \mathrm{Z}$ is the pull-back of the principal G-bundle $\left(\mathrm{Y} ; \mathrm{m}_{\mathrm{Y}} ; \mathrm{s}_{\mathrm{Y}}\right)$ over $\mathrm{p}_{\mathrm{Y}}: \mathrm{Y} \rightarrow \mathrm{Z}$ along $\mathrm{pr}_{2}: \mathrm{B} \times_{f, \mathrm{~A}, \alpha} \mathrm{Z} \rightarrow \mathrm{Z}$. Therefore, it is a partially principal bundle. It is clear that under Assumption 2.15 the arrow $\left(\mathrm{pr}_{1} ; \mathrm{pY}_{\mathrm{Y}} \circ \mathrm{pr}_{2}\right): \mathrm{X} \rightarrow \mathrm{B} \times_{f, \mathrm{~A}, \alpha} \mathrm{Z}$ is a cover and, therefore, the G-bundle $\left(\mathrm{X} ; \mathrm{m}_{\mathrm{X}} ; \mathrm{sX}\right)$ over $\left(\mathrm{pr}_{1} ; \mathrm{p}_{\mathrm{Y}} \circ \mathrm{pr}_{2}\right): \mathrm{X} \rightarrow \mathrm{B} \times_{f, \mathrm{~A}, \alpha} \mathrm{Z}$ is principal.

Lemma 5.51. Let $\left(\mathrm{X} ; \mathrm{m}_{\mathrm{X}} ; \mathrm{s}_{\mathrm{X}}\right)$ be a principal $\mathrm{G}$-bundle over $\mathrm{p}_{\mathrm{X}}: \mathrm{X} \rightarrow \mathrm{Z}_{\mathrm{X}}$ and let $\left(\mathrm{Y} ; \mathrm{m}_{\mathrm{Y}} ; \mathrm{s}_{\mathrm{Y}}\right)$ be a partially principal $\mathrm{G}$-bundle over $\mathrm{p}_{\mathrm{Y}}: \mathrm{Y} \rightarrow \mathrm{Z}_{\mathrm{Y}}$. Any $\mathrm{G}-\mathrm{map}$ $f: \mathrm{X} \rightarrow \mathrm{Y}$ induces an arrow $\tilde{f}: \mathrm{Z}_{\mathrm{X}} \rightarrow \mathrm{Z}_{\mathrm{Y}}$ such that $\tilde{f} \circ \mathrm{p}_{\mathrm{X}}=\mathrm{p}_{\mathrm{Y}} \circ f$.

Proof. Let $f: X \rightarrow Y$ be a G-map. We know that $\mathrm{p}_{\mathbf{X}}: \mathrm{X} \rightarrow \mathrm{Z}_{\mathrm{X}}$ is a coequaliser of the pair of parallel arrows $\mathrm{pr}_{1}, \mathrm{~m}_{\mathrm{X}}: \mathrm{X} \times_{\mathrm{sX}_{\mathrm{X}}, \mathrm{G}^{0}, \mathrm{r}} \mathrm{G}^{1} \rightrightarrows \mathrm{X}$, and

$$
\mathrm{p}_{\mathrm{Y}} \circ f \circ \mathrm{pr}_{1}=\mathrm{pY}_{\mathrm{Y}} \circ \mathrm{pr}_{1} \circ\left(f \circ \mathrm{pr}_{1} ; \mathrm{pr}_{2}\right)=\mathrm{p}_{\mathrm{Y}} \circ \mathrm{m}_{\mathrm{Y}} \circ\left(f \circ \mathrm{pr}_{1} ; \mathrm{pr}_{2}\right)=\mathrm{p}_{\mathrm{Y}} \circ f \circ \mathrm{m}_{\mathbf{X}} \text {. }
$$

Therefore, there exists an arrow $\tilde{f}: Z_{X} \rightarrow Z_{Y}$ which is a factorization of the arrow $\mathrm{p}_{\boldsymbol{\gamma}} \circ f: \mathrm{X} \rightarrow \mathrm{Z}_{\mathrm{Y}}$. Hence $\tilde{f} \circ \mathrm{p}_{\mathbf{X}}=\mathrm{p}_{\mathrm{Y}} \circ f$. The arrow $\tilde{f}$ is defined elementwise by $\tilde{f}([x])=[f(x)]$ for all $x \in \mathbf{X}$.

The equality in Lemma 5.51 means that the diagram

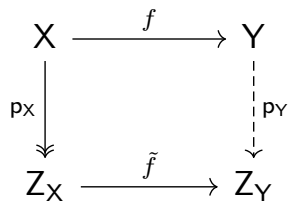


commutes. Under Assumption 2.15 it is a pull-back square by Proposition 5.48 The following corollaries give extra information about the arrows $f: \mathrm{X} \rightarrow \mathrm{Y}$ and $\tilde{f}: Z_{X} \rightarrow Z_{Y}$ under additional assumptions.

Corollary 5.52. Under Assumption 2.15, if $\tilde{f}: \mathrm{Z}_{X} \rightarrow \mathrm{Z}_{Y}$ is a partial cover, so is $f: \mathrm{X} \rightarrow \mathrm{Y}$, and if $\tilde{f}: \mathrm{Z}_{\mathrm{X}} \rightarrow \mathrm{Z}_{\mathrm{Y}}$ is a cover, so is $f: \mathrm{X} \rightarrow \mathrm{Y}$.

Proof. We just use Proposition 5.48 and Assumption 2.15 and the proof is obvious.

Corollary 5.53. Assume Assumptions 2.15 and 2.18 . Let $\left(\mathrm{X} ; \mathrm{m}_{\mathrm{X}} ; \mathrm{s}_{\mathrm{X}}\right)$ and $\left(\mathrm{Y} ; \mathrm{m}_{\mathrm{Y}} ; \mathrm{s}_{\mathrm{Y}}\right)$ be principal $\mathrm{G}$-bundles over $\mathrm{p}_{\mathrm{X}}: \mathrm{X} \rightarrow \mathrm{Z}_{\mathrm{X}}$ and $\mathrm{p}_{\mathrm{Y}}: \mathrm{Y} \rightarrow \mathrm{Z}_{\mathrm{Y}}$, respectively, and let $f: \mathrm{X} \rightarrow \mathrm{Y}$ be a $\mathrm{G}-$ map that induces the arrow $\tilde{f}: \mathbf{Z}_{\mathrm{X}} \rightarrow \mathbf{Z}_{\mathrm{Y}} . f: \mathrm{X} \rightarrow \mathrm{Y}$ is a cover if and only if $\tilde{f}: Z_{X} \rightarrow Z_{Y}$ is.

Proof. If $\tilde{f}: Z_{X} \rightarrow Z_{Y}$ is a cover, then so is $f: X \rightarrow Y$ because of Corollary 5.52 If $f: X \rightarrow Y$ is a cover, then so is $\tilde{f}: Z_{X} \rightarrow Z_{Y}$ because of Assumption 2.18.

Corollary 5.54. Under Assumption 2.15, $\tilde{f}: Z_{X} \rightarrow Z_{Y}$ is an isomorphism if and only if $f: \mathrm{X} \rightarrow \mathrm{Y}$ is.

Proof. Since $\mathrm{p}_{\mathrm{X}}: \mathrm{X} \rightarrow \mathrm{Z}_{\mathrm{X}}$ is a cover, it is epic. Therefore, Lemma 2.13 works. The converse holds in any pull-back square.

5.6. Basic actions and assumptions on it. In this subsection, we define basic and partially basic actions and consider an extra assumption about basic actions. Let $\mathrm{G}$ be a groupoid in the category $\left(\mathcal{C}, \mathcal{F}_{p}\right)$ with partial covers.

Definition 5.55. A G-action $\left(\mathrm{X} ; \mathrm{m}_{\mathrm{X}} ; \mathrm{s}_{\mathrm{X}}\right)$ is called partially basic if it is a partially principal G-bundle over some partial cover $\mathrm{p}: \mathrm{X} \rightarrow \mathrm{Z}$. A partially basic action is basic if this G-bundle is principal.

Proposition 5.56. A G-action $\left(\mathrm{X} ; \mathrm{m}_{\mathrm{X}} ; \mathrm{s}_{\mathrm{X}}\right)$ is partially basic if and only if the tranformation groupoid $\mathrm{X} \rtimes \mathrm{G}$ is isomorphic to a Čech groupoid of some partial cover $\mathrm{p}: \mathrm{X} \rightarrow \mathrm{Z}$.

Here we mean that the isomorphism between these groupoids is an identity arrow on objects.

Proof. Suppose that the G-action $\left(\mathrm{X} ; \mathrm{m}_{\mathbf{X}} ; \mathrm{s}_{\mathbf{X}}\right)$ is partially basic. That is, it is a partially principal G-bundle over a partial cover $\mathrm{p}: \mathbf{X} \rightarrow \mathbf{Z}$. We are going to show that the transformation groupoid is isomorphic to the Čech groupoid of $\mathrm{p}: \mathrm{X} \rightarrow \mathrm{Z}$. Notice that the pair of arrows $\left(\mathrm{pr}_{1} ; \mathrm{m}_{\mathrm{X}}\right): \mathrm{X} \times_{\mathrm{s}_{\mathrm{X}}, \mathrm{G}^{0}, \mathrm{r}} \mathrm{G}^{1} \rightarrow \mathrm{X} \times \times_{\mathrm{p}, Z, \mathrm{p}} \mathrm{X}$ and id ${ }_{X}: X \rightarrow X$ gives the functor from the transformation groupoid to the Cech groupoid of $\mathrm{p}: \mathrm{X} \rightarrow \mathrm{Z}$. The equalities $\mathrm{pr}_{1} \circ\left(\mathrm{pr}_{1} ; \mathrm{m}_{\mathrm{X}}\right)=\mathrm{pr}_{1}$ and $\mathrm{pr}_{2} \circ\left(\mathrm{pr}_{1} ; \mathrm{m}_{\mathrm{X}}\right)=\mathrm{m}_{\mathrm{X}}$ mean that $\left(\mathrm{pr}_{1} ; \mathrm{m}_{\mathrm{X}}\right)$ and $\mathrm{id} \mathrm{X}$ intertwine the source and range maps of these groupoids. We need to check it for the multiplication maps. We have

$$
\begin{aligned}
\left(\mathrm{pr}_{1} ; \mathrm{m}_{\mathbf{X}}\right)\left((x ; g) \cdot\left(x_{1} ; g_{1}\right)\right) & =\left(\mathrm{pr}_{1} ; \mathrm{m}_{\mathbf{X}}\right)\left(x ; g \cdot g_{1}\right) \\
& =\left(x ; x \cdot\left(g \cdot g_{1}\right)\right) \\
& =\left(x ;(x \cdot g) \cdot g_{1}\right) \\
& =\left(x ; x_{1} \cdot g_{1}\right) \\
& =\left(x ; x_{1}\right) \cdot\left(x_{1} ; x_{1} \cdot g_{1}\right) \\
& =(x ; x \cdot g) \cdot\left(x_{1} ; x_{1} \cdot g_{1}\right) \\
& =\left(\mathrm{pr}_{1} ; \mathrm{m}_{\mathbf{X}}\right)(x ; g) \cdot\left(\mathrm{pr}_{1} ; \mathrm{m}_{\mathbf{X}}\right)\left(x_{1} ; g_{1}\right)
\end{aligned}
$$

for all $x, x_{1} \in \mathrm{X}, g, g_{1} \in \mathrm{G}^{1}$ with $\mathrm{s}_{\mathbf{X}}(x)=\mathrm{r}(g)$, sX $\left(x_{1}\right)=\mathrm{r}\left(g_{1}\right)$ and $x \cdot g=x_{1}$. So the multiplication maps are intertwined, too. Since $\left(X ; m_{X} ; s_{X}\right)$ is a partially 
principal G-bundle over $\mathrm{p}: \mathrm{X} \rightarrow \mathrm{Z}$, the arrow $\left(\mathrm{pr}_{1} ; \mathrm{m}_{\mathrm{X}}\right)$ is invertible. So we have an isomorphism between the transformation groupoid $X \rtimes G$ and the Čech groupoid of $\mathrm{p}: \mathrm{X} \rightarrow \mathrm{Z}$.

Conversely, let the transformation groupoid be isomorphic to the Čech groupoid of $p: X \rightarrow Z$. We assume that this isomorphism is an identity arrow on objects and an isomorphism $\varphi: X \times_{\mathrm{sX}_{\mathrm{X}} \mathrm{G}^{0}, \mathrm{r}} \mathrm{G}^{1} \rightarrow \mathrm{X} \times_{\mathrm{p}, \mathrm{Z}, \mathrm{p}} \mathrm{X}$ on arrows. Since it has to intertwine the source and range maps, we have $\mathrm{pr}_{1} \circ \varphi=\mathrm{pr}_{1}$ and $\mathrm{pr}_{2} \circ \varphi=\mathrm{m}_{\mathrm{X}}$. Therefore, $\varphi=\left(\mathrm{pr}_{1} ; \mathrm{m}_{\mathrm{X}}\right)$. So $\left(\mathrm{pr}_{1} ; \mathrm{m}_{\mathrm{X}}\right): \mathrm{X} \times_{\mathrm{s}_{\mathrm{X}}, \mathrm{G}^{0}, \mathrm{r}} \mathrm{G}^{1} \rightarrow \mathrm{X} \times_{\mathrm{p}, \mathrm{Z}, \mathrm{p}} \mathrm{X}$ is invertible. Thus the $\mathrm{G}$-action $\left(\mathrm{X} ; \mathrm{m}_{\mathrm{X}} ; \mathrm{s}_{\mathrm{X}}\right)$ is partially basic.

Remark 5.57. The global case of Proposition 5.56 with its proof is absolutely the same. A G-action $\left(X ; m_{X} ; s_{X}\right)$ is basic if and only if the tranformation groupoid $X \rtimes G$ is isomorphic to a Čech groupoid of some cover $\mathrm{p}: \mathrm{X} \rightarrow \mathbf{Z}$.

Definition 5.58. A groupoid G is called partially basic or basic if the canonical action on its objects (described in Example 5.21) is partially basic or basic, respectively.

Lemma 5.59. A groupoid $\mathrm{G}=\left(\mathrm{G}^{0}, \mathrm{G}^{1}, \mathrm{r}, \mathrm{s}, \mathrm{m}\right)$ is partially basic if and only if there is a partial cover $\mathrm{p}: \mathrm{G}^{0} \rightarrow \mathrm{Z}$ such that the arrow $(\mathrm{r} ; \mathrm{s}): \mathrm{G}^{1} \rightarrow \mathrm{G}^{0} \times_{\mathrm{p}, \mathrm{Z}, \mathrm{p}} \mathrm{G}^{0}$ is well-defined and invertible.

Proof. Suppose that a groupoid G is partially basic. That is, the action described in Example 5.21 is partially basic. Therefore, there is a G-invariant partial

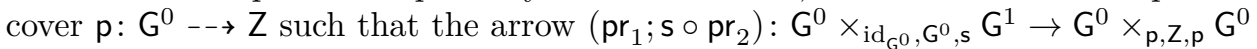
is invertible. The arrow $\mathrm{G}^{0} \times \times_{\mathrm{id}_{\mathrm{G}^{0}}, \mathrm{G}^{0}, \mathrm{~s}} \mathrm{G}^{1} \rightarrow \mathrm{G}^{1}$ is an isomorphism because it is a pull-back of $\mathrm{id}_{\mathrm{G}^{0}}$. Also, $\mathrm{pr}_{1}=\mathrm{r} \circ \mathrm{pr}_{2}$. Therefore, $(\mathrm{r} ; \mathrm{s})=\left(\mathrm{pr}_{1} ; \mathrm{s} \circ \mathrm{pr}_{2}\right) \circ \mathrm{pr}_{2}^{-1}$. Thus the arrow $(r ; s): G^{1} \rightarrow G^{0} \times_{p, Z, p} G^{0}$ is well-defined and invertible.

Conversely, if the arrow $(r ; s): G^{1} \rightarrow G^{0} \times_{p, Z, p} G^{0}$ is well-defined and invertible, then the arrow $\left(\mathrm{pr}_{1}\right.$; s $\left.\circ \mathrm{pr}_{2}\right): \mathrm{G}^{0} \times_{\mathrm{id}_{\mathrm{G}}, \mathrm{G}^{0}, \mathrm{~s}} \mathrm{G}^{1} \rightarrow \mathrm{G}^{0} \times_{\mathrm{p}, \mathrm{Z}, \mathrm{p}} \mathrm{G}^{0}$ is well-defined and invertible because $\left(\mathrm{pr}_{1} ; \mathrm{s} \circ \mathrm{pr}_{2}\right)=(\mathrm{r} ; \mathrm{s}) \circ \mathrm{pr}_{2}$.

It is clear that we have the same in the case of the global situation.

Corollary 5.60. A groupoid $\mathrm{G}=\left(\mathrm{G}^{0}, \mathrm{G}^{1}, \mathrm{r}, \mathrm{s}, \mathrm{m}\right)$ is partially basic if and only if it is isomorphic to the Čech groupoid of some partial cover $\mathrm{p}: \mathrm{G}^{0} \rightarrow-\rightarrow \mathrm{Z}$.

Here we mean that the isomorphism between these groupoids is an identity arrow on objects.

Proof. If $\mathrm{G}$ is partially basic then there are a partial cover $\mathrm{p}: \mathrm{G}^{0} \rightarrow \mathrm{Z}$ and a welldefined isomorphism $(r ; s): G^{1} \rightarrow G^{0} \times_{p, Z, p} G^{0}$. This isomorphism and the identity arrow on objects intertwine the range and source maps of the groupoid $G$ and the Čech groupoid of $\mathrm{p}: \mathrm{G}^{0} \rightarrow-\rightarrow$ Z. They intertwine the multiplication maps, too because we have

$$
\begin{aligned}
(\mathrm{r} ; \mathrm{s})\left(g \cdot g_{1}\right) & =\left(\mathrm{r}\left(g \cdot g_{1}\right) ; \mathrm{s}\left(g \cdot g_{1}\right)\right) \\
& =\left(\mathrm{r}(g) ; \mathrm{s}\left(g_{1}\right)\right) \\
& =(\mathrm{r}(g) ; \mathrm{s}(g)) \cdot\left(\mathrm{s}(g) ; \mathrm{s}\left(g_{1}\right)\right) \\
& =(\mathrm{r}(g) ; \mathrm{s}(g)) \cdot\left(\mathrm{r}\left(g_{1}\right) ; \mathrm{s}\left(g_{1}\right)\right) \\
& =(\mathrm{r} ; \mathrm{s})(g) \cdot(\mathrm{r} ; \mathrm{s})\left(g_{1}\right)
\end{aligned}
$$

for all $g, g_{1} \in \mathrm{G}^{1}$ with $\mathrm{s}(g)=\mathrm{r}\left(g_{1}\right)$. Therefore, we have an isomorphism between the groupoid $G$ and the Čech groupoid of $p: G^{0} \rightarrow Z$. 
Remark 5.61. There is an analogous corollary in the global case: A groupoid $\mathrm{G}=\left(\mathrm{G}^{0}, \mathrm{G}^{1}, \mathrm{r}, \mathrm{s}, \mathrm{m}\right)$ is basic if and only if it is isomorphic to the Čech groupoid of some cover $\mathrm{p}: \mathrm{G}^{0} \rightarrow \mathrm{Z}$.

Corollary 5.62. A G-action $\left(\mathrm{X} ; \mathrm{m}_{\mathrm{X}} ; \mathrm{s}_{\mathrm{X}}\right)$ is partially basic if and only if the transformation groupoid $\mathrm{X} \rtimes \mathrm{G}$ is partially basic. A $\mathrm{G}$-action $\left(\mathrm{X} ; \mathrm{m}_{\mathbf{X}} ; \mathrm{s}_{\mathbf{X}}\right)$ is basic if and only if the tranformation groupoid $\mathrm{X} \rtimes \mathrm{G}$ is basic.

Proof. This follows from Proposition 5.56 and Corollary 5.60

The following useful lemmas cannot be proven without the following extra assumption about basic groupoids and basic actions.

Assumption 5.63. Any action of a basic groupoid is basic.

Lemma 5.64. Let $\left(\mathrm{X} ; \mathrm{m}_{\mathrm{X}} ; \mathrm{sX}_{\mathrm{X}}\right)$ and $\left(\mathrm{Y} ; \mathrm{m}_{\mathrm{Y}} ; \mathrm{s}_{\mathrm{Y}}\right)$ be a $\mathrm{G}$-actions and let $f: \mathrm{Y} \rightarrow \mathrm{X}$ be a G-map. Under Assumption 5.63, if $\left(\mathrm{X} ; \mathrm{m}_{\mathrm{X}} ; \mathrm{s}_{\mathrm{X}}\right)$ is basic then so is $\left(\mathrm{Y} ; \mathrm{m}_{\mathrm{Y}} ; \mathrm{s}_{\mathrm{Y}}\right)$.

Proof. Since the $\mathrm{G}$-action $\left(\mathrm{X} ; \mathrm{m}_{\mathrm{X}} ; \mathrm{s}_{\mathrm{X}}\right)$ is basic, the transformation groupoid $\mathrm{X} \rtimes \mathrm{G}$ is basic by Corollary 5.62 The G-action ( $\mathrm{Y} ; \mathrm{m}_{\mathrm{Y}} ; \mathrm{s}_{\mathrm{Y}}$ ) together with a G-map $f: \mathrm{Y} \rightarrow \mathrm{X}$ is equivalent to an $\mathrm{X} \rtimes \mathrm{G}$-action over $\mathrm{Y}$ by Proposition 5.32 Therefore, the G-action $\left(\mathrm{Y} ; \mathrm{m}_{\mathrm{Y}} ; \mathrm{s}_{\mathrm{Y}}\right)$ is basic by Assumption 5.63 .

Lemma 5.65. Assume Assumptions 2.15 and 5.63. Let $\left(\mathrm{X} ; \mathrm{m}_{\mathrm{X}} ; \mathrm{sX}_{\mathrm{X}}\right),\left(\mathrm{X}_{1} ; \mathrm{m}_{\mathrm{X}_{1}} ; \mathrm{s}_{\mathrm{X}_{1}}\right)$ and $\left(\mathrm{X}_{2} ; \mathrm{m}_{\mathrm{X}_{2}} ; \mathrm{s}_{\mathrm{X}_{2}}\right)$ be principal $\mathrm{G}$-bundles over $\mathrm{p}: \mathrm{X} \rightarrow \mathrm{Z}, \mathrm{p}_{1}: \mathrm{X}_{1} \rightarrow \mathrm{Z}_{1}$ and $\mathrm{p}_{2}: \mathrm{X}_{2} \rightarrow$ $\mathrm{Z}_{2}$, respectively. Let $f_{1}: \mathrm{X}_{1} \rightarrow \mathrm{X}$ and $f_{2}: \mathrm{X}_{2} \rightarrow \mathrm{X}$ be $\mathrm{G}-$ maps and let them induce $\tilde{f}_{1}: \mathbf{Z}_{1} \rightarrow \mathbf{Z}$ and $\tilde{f}_{2}: \mathbf{Z}_{2} \rightarrow \mathbf{Z}$, respectively. If the fibre products $\mathbf{X}_{1} \times_{f_{1}, \mathrm{X}_{f_{2}}} \mathbf{X}_{2}$ and $\mathrm{Z}_{1} \times \tilde{f}_{1}, \mathrm{Z}, \tilde{f}_{2} \mathbf{Z}_{2}$ exist, then the fibre product of $\mathrm{G}$-maps $f_{1}: \mathrm{X}_{1} \rightarrow \mathrm{X}$ and $f_{2}: \mathbf{X}_{2} \rightarrow \mathbf{X}$, discribed in Lemma 5.20, is a principal G-bundle over

$$
\left(\mathrm{p}_{1} \circ \mathrm{pr}_{1} ; \mathrm{p}_{2} \circ \mathrm{pr}_{2}\right): \mathrm{X}_{1} \times_{f_{1}, \mathrm{X}, f_{2}} \mathrm{X}_{2} \rightarrow \mathrm{Z}_{1} \times_{\tilde{f_{1}}, \mathrm{Z}, \tilde{f_{2}}} \mathrm{Z}_{2}, \quad\left(x_{1} ; x_{2}\right) \mapsto\left(\left[x_{1}\right] ;\left[x_{2}\right]\right) .
$$

Proof. We know by construction that the coordinate projections $\mathrm{pr}_{1}$ and $\mathrm{pr}_{2}$ of $\mathrm{X}_{1} \times_{f_{1}, \mathrm{X}, f_{2}} \mathrm{X}_{2}$ are G-maps (see Lemma 5.20). So the G-action $\left(\mathrm{X}_{1} \times_{f_{1}, \mathrm{X}, f_{2}} \mathrm{X}_{2} ; \mathrm{m}_{0} ; \mathrm{s}_{0}\right)$ is a principal G-bundle over some cover $\mathrm{p}_{12}: \mathrm{X}_{1} \times_{f_{1}, \mathrm{X}, f_{2}} \mathrm{X}_{2} \rightarrow \mathrm{Z}_{12}$ by Lemma 5.64. Let $\tilde{\mathrm{pr}}_{1}: \mathrm{Z}_{12} \rightarrow \mathrm{Z}_{1}$ and $\tilde{\mathrm{pr}}_{2}: \mathrm{Z}_{12} \rightarrow \mathrm{Z}_{2}$ be arrows induced by $\mathrm{pr}_{1}$ and $\mathrm{pr}_{2}$, respectively. We must show that $Z_{12} \cong Z_{1} \times \tilde{f}_{1}, \mathrm{Z}, \tilde{f}_{2} Z_{2}$ and $\mathrm{p}_{12}=\left(\mathrm{p}_{1} \circ \mathrm{pr}_{1} ; \mathrm{p}_{2} \circ \mathrm{pr}_{2}\right)$.

Consider any object $A$ and arrows $z_{1}: A \rightarrow Z_{1}$ and $z_{2}: A \rightarrow Z_{2}$ such that $\tilde{f}_{1} \circ z_{1}=\tilde{f}_{2} \circ z_{2}$. Let G-action $(\mathrm{B} ; \mathrm{m} ; \mathrm{s})$ over $\alpha: \mathrm{B} \rightarrow \mathrm{A}$ be a pull-back of the principal G-bundle $\left(\mathbf{X} ; \mathrm{m}_{\mathbf{X}} ; \mathrm{s}_{\mathbf{X}}\right)$ over $\mathrm{p}: \mathbf{X} \rightarrow \mathbf{Z}$ along the arrow $\tilde{f}_{1} \circ z_{1}=\tilde{f}_{2} \circ z_{2}$. On the one hand it is a pull-back of the principal G-bundle $\left(\mathrm{X}_{1} ; \mathrm{m}_{\mathrm{X}_{1}} ; \mathrm{s}_{\mathrm{X}_{1}}\right)$ over $\mathrm{p}_{1}: \mathrm{X}_{1} \rightarrow \mathrm{Z}_{1}$ along the arrow $z_{1}: A \rightarrow Z_{1}$ and on the other hand it is a pull-back of the principal G-bundle $\left(\mathrm{X}_{2} ; \mathrm{m}_{\mathrm{X}_{2}} ; \mathrm{s}_{\mathrm{X}_{2}}\right)$ over $\mathrm{p}_{2}: \mathrm{X}_{2} \rightarrow \mathrm{Z}_{2}$ along the arrow $z_{2}: \mathrm{A} \rightarrow \mathrm{Z}_{2}$. Therefore, there are G-maps $x_{1}: \mathrm{B} \rightarrow \mathrm{X}_{1}$ and $x_{2}: \mathrm{B} \rightarrow \mathrm{X}_{2}$ which induce the arrows $z_{1}: \mathrm{A} \rightarrow \mathrm{Z}_{1}$ and $z_{2}: \mathrm{A} \rightarrow \mathrm{Z}_{2}$, respectively. Since the lifting of the arrow $\tilde{f}_{1} \circ z_{1}=\tilde{f}_{2} \circ z_{2}$ is unique, we have $f_{1} \circ x_{1}=f_{2} \circ x_{2}$. Thus there is a unique arrow $\left(x_{1} ; x_{2}\right): \mathrm{B} \rightarrow \mathrm{X}_{1} \times_{f_{1}, \mathrm{X}, f_{2}} \mathrm{X}_{2}$ which is a G-map by Lemma 5.20 . Therefore, it induces a unique arrow $z_{12}: \mathrm{A} \rightarrow \mathrm{Z}_{12}$ such that $z_{12} \circ \alpha=\mathrm{p}_{12} \circ\left(x_{1} ; x_{2}\right)$. Since $\operatorname{pr}_{1} \circ\left(x_{1} ; x_{2}\right)=x_{1}$ and $\operatorname{pr}_{2} \circ\left(x_{1} ; x_{2}\right)=x_{2}$, we have $\tilde{\mathrm{pr}}_{1} \circ z_{12}=z_{1}$ and $\tilde{\mathrm{pr}}_{2} \circ z_{12}=z_{2}$. By construction, such arrow $z_{12}$ is unique, and therefore, we have $Z_{12} \cong Z_{1} \times_{\tilde{f}_{1}, Z, \tilde{f}_{2}} Z_{2}$. Since $\tilde{p r}_{1} \circ p_{12}=p_{1} \circ p_{1}$ and $\tilde{p r}_{2} \circ p_{12}=p_{2} \circ p_{2}$, we can deduce that the fibre product of the G-maps $f_{1}: \mathbf{X}_{1} \rightarrow \mathbf{X}$ and $f_{2}: \mathbf{X}_{2} \rightarrow \mathrm{X}$ is a principal G-bundle over $\left(\mathrm{p}_{1} \circ \mathrm{pr}_{1} ; \mathrm{p}_{2} \circ \mathrm{pr}_{2}\right): \mathrm{X}_{1} \times_{f_{1}, \mathrm{X}, f_{2}} \mathrm{X}_{2} \rightarrow \mathrm{Z}_{1} \times_{\tilde{f}_{1}, \mathrm{Z}, \tilde{f}_{2}} \mathrm{Z}_{2}$.

There is a weaker assumption about basic groupoids and basic action.

Assumption 5.66. Any sheaf over a basic groupoid is basic.

Lemma 5.67. Let $\left(\mathrm{X} ; \mathrm{m}_{\mathrm{X}} ; \mathrm{s}_{\mathrm{X}}\right)$ and $\left(\mathrm{Y} ; \mathrm{m}_{\mathrm{Y}} ; \mathrm{s}_{\mathrm{Y}}\right)$ be $\mathrm{G}$-actions and let the cover $f: \mathrm{Y} \rightarrow$ $\mathrm{X}$ be a $\mathrm{G}$-map. Under Assumption 5.66, if $\left(\mathrm{X} ; \mathrm{m}_{\mathbf{X}} ; \mathrm{s}_{\mathrm{X}}\right)$ is basic then so is $\left(\mathrm{Y} ; \mathrm{m}_{\mathrm{Y}} ; \mathrm{s}_{\mathrm{Y}}\right)$. 
Proof. Since the G-action $\left(\mathrm{X} ; \mathrm{m}_{\mathrm{X}} ; \mathrm{s}_{\mathrm{X}}\right)$ is basic, the transformation groupoid $\mathrm{X} \rtimes \mathrm{G}$ is basic by Corollary 5.62 The G-action $\left(\mathrm{Y} ; \mathrm{m}_{\mathrm{Y}} ; \mathrm{s}_{\mathrm{Y}}\right)$ together with a G-map $f: \mathrm{Y} \rightarrow \mathrm{X}$ is equivalent to anX $\rtimes \mathrm{G}$-action on $\mathrm{Y}$ by Proposition 5.32. Therefore, the G-action $\left(\mathrm{Y} ; \mathrm{m}_{\mathrm{Y}} ; \mathrm{s}_{\mathrm{Y}}\right)$ is basic by Assumption 5.63 .

\subsection{Groupoid fibrations with basic fibre.}

Proposition 5.68. Assume Assumption 2.15, Let $\mathrm{F}: \mathrm{L} \rightarrow \mathrm{H}$ be a groupoid fibration with basic fibre $\mathrm{G}$. There is a groupoid $\mathrm{L} / \mathrm{G}$, call it a quotient groupoid, with a canonical groupoid fibration $\mathrm{F}_{1}: \mathrm{L} \rightarrow \mathrm{L} / \mathrm{G}$ with fibre $\mathrm{G}$, which is a cover on objects, and with a quotient groupoid covering $\mathrm{F}_{2}: \mathrm{L} / \mathrm{G} \rightarrow \mathrm{H}$ such that $\mathrm{F}=\mathrm{F}_{2} \circ \mathrm{F}_{1}$.

Proof. Since the groupoid $\mathrm{G}$ is basic, there are an object $\mathbf{Z}$ and a cover $\mathrm{p}: \mathrm{L}^{0} \rightarrow \mathbf{Z}$ which are an orbit space and orbit space projection of the right canonical action of $\mathrm{G}$ on its objects $\mathrm{L}^{0}$ (see Example 5.21). It is clear that the left canonical action of $\mathrm{G}$ on its objects $\mathrm{L}^{0}$ has the same orbit space and orbit space projection.

Notice that the arrow $\mathrm{F}^{0}: \mathrm{L}^{0} \rightarrow \mathrm{H}^{0}$ is G-invariant because

$$
\begin{aligned}
\mathrm{F}^{0}\left(l_{0} \cdot g\right) & =\mathrm{F}^{0}\left(\mathrm{~s}_{\mathrm{L}}(g)\right) \\
& =\mathrm{r}_{\mathrm{H}}\left(1_{\mathrm{F}^{0}\left(\mathrm{~s}_{\mathrm{L}}(g)\right)}\right) \\
& =\mathrm{r}_{\mathrm{H}}\left(\mathrm{F}^{1}(g)\right) \\
& =\mathrm{F}^{0}\left(\mathrm{r}_{\mathrm{L}}(g)\right) \\
& =\mathrm{F}^{0}\left(l_{0}\right)
\end{aligned}
$$

for all $l_{0} \in \mathrm{L}^{0}, g \in \mathrm{G}^{1}$ with $l_{0}=\mathrm{r}_{\mathrm{L}}(g)$. Thus we have a unique arrow $\alpha: \mathrm{Z} \rightarrow \mathrm{H}^{0}$ such that $\mathrm{F}^{0}=\alpha \circ \mathrm{p}$. Elementwise $\alpha\left(\left[l_{0}\right]\right)=\mathrm{F}^{0}\left(l_{0}\right)$ for all $l_{0} \in \mathrm{L}^{0}$.

There is a left principal G-bundle $\left(\mathrm{L}^{1} ; \mathrm{m} ; \mathrm{r}_{\mathrm{L}}\right)$ over the arrow

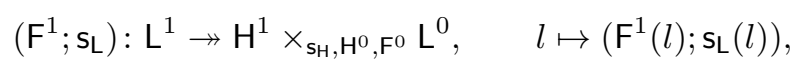

which is described in Example 5.27. Also, we have a canonical left principal Gbundle over $p: L^{0} \rightarrow Z$. Let us show that the range map $r_{L}: L^{1} \rightarrow L^{0}$ is a $G$-map. It obviously commutes with anchor maps. We also have

$$
\begin{aligned}
\mathrm{r}_{\mathrm{L}}(g \cdot l) & =\mathrm{r}_{\mathrm{L}}(g) \\
& =g \cdot \mathrm{s}_{\mathrm{L}}(g) \\
& =g \cdot \mathrm{r}_{\mathrm{L}}(l)
\end{aligned}
$$

for all $g \in \mathrm{G}^{1}, l \in \mathrm{L}^{1}$ with $\mathrm{s}_{\mathrm{L}}(g)=\mathrm{r}_{\mathrm{L}}(l)$. Therefore, the $\mathrm{G}$-map $\mathrm{r}_{\mathrm{L}}: \mathrm{L}^{1} \rightarrow \mathrm{L}^{0}$ induces

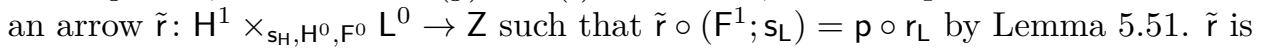
defined elementwise by $\tilde{\mathrm{r}}\left(\mathrm{F}^{1}(l) ; \mathrm{s}_{\mathrm{L}}(l)\right)=\left[\mathrm{r}_{\mathrm{L}}(l)\right]$ for all $l \in \mathrm{L}^{1}$.

Consider the pull-back of the right G-bundle $\left(\mathrm{L}^{0} ; \mathrm{s}_{\mathrm{G}} \circ \mathrm{pr}_{2} ; \mathrm{id}_{\mathrm{L}^{0}}\right)$ over $\mathrm{F}^{0}: \mathrm{L}^{0} \rightarrow \mathrm{H}^{0}$ along the cover $\mathrm{s}_{\mathrm{H}}: \mathrm{H}^{1} \rightarrow \mathrm{H}^{0}$. An action map of this action is defined elementwise by $\left(h ; l_{0}\right) \cdot g=\left(h ; \mathrm{s}_{\mathrm{L}}(g)\right)$ for all $h \in \mathrm{H}^{1}, l_{0} \in \mathrm{L}^{0}, g \in \mathrm{G}^{1}$ with $\mathrm{s}_{\mathrm{H}}(h)=\mathrm{F}^{0}\left(l_{0}\right)$ and $l_{0}=\mathrm{r}_{\mathrm{L}}(g)$. We know that, under Assumption 2.15 this action is a principal G-bundle

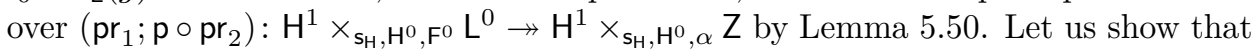

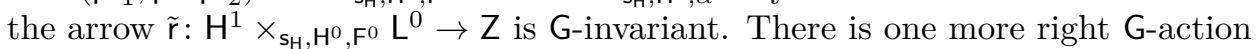
defined in Remark 5.28 That is $\left(\mathrm{L}^{1} ; \mathrm{m}_{2} ; \mathrm{s}\right)$, where $\mathrm{m}_{2}=\mathrm{m}_{\mathrm{L}} \circ\left(\mathrm{pr}_{1} ; \mathrm{pr}_{1} \circ \mathrm{pr}_{2}\right)$. In the 
diagram

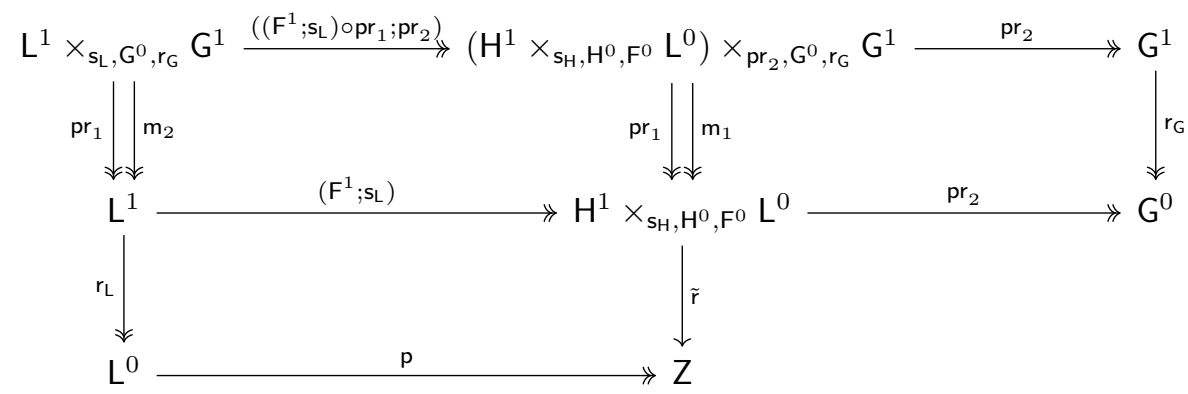

each square is a pull-back square. The arrow $\left(\left(\mathrm{F}^{1} ; \mathrm{s}_{\mathrm{L}}\right) \circ \mathrm{pr}_{1} ; \mathrm{pr}_{2}\right)$ is a cover by Assumption 2.15 because it is a pull-back of $\left(\mathrm{F}^{1} ; \mathrm{s}_{\mathrm{L}}\right)$. So it is a coequaliser and therefore, it is epic. For any $l$ in $\mathrm{L}^{1}$ and for any $g$ in $\mathrm{G}^{1}$ with $\mathrm{s}_{\mathrm{L}}(l)=\mathrm{r}_{\mathrm{L}}(g)$ we have

$$
\begin{aligned}
\tilde{\mathrm{r}}\left(\left(\mathrm{F}^{1}(l) ; \mathrm{s}_{\mathrm{L}}(l)\right) \cdot g\right) & =\tilde{\mathrm{r}}\left(\mathrm{F}^{1}(l) ; \mathrm{r}_{\mathrm{L}}(g)\right) \\
& =\tilde{\mathrm{r}}\left(\mathrm{F}^{1}(l) ; \mathrm{s}_{\mathrm{L}}(l)\right) \\
& =\left[\mathrm{r}_{\mathrm{L}}(l)\right] \\
& =\tilde{\mathrm{r}}\left(\mathrm{F}^{1}(l) ; \mathrm{s}_{\mathrm{L}}(l)\right) .
\end{aligned}
$$

Therefore, we have $\tilde{r} \circ \mathrm{m}_{1} \circ\left(\left(\mathrm{F}^{1} ; \mathrm{sL}_{\mathrm{L}}\right) \circ \mathrm{pr}_{1} ; \mathrm{pr}_{2}\right)=\tilde{\mathrm{r}} \circ \mathrm{pr}_{1} \circ\left(\left(\mathrm{F}^{1} ; \mathrm{sL}_{\mathrm{L}}\right) \circ \mathrm{pr}_{1} ; \mathrm{pr}_{2}\right)$. Since

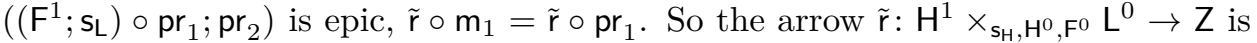
G-invariant. Therefore, there is a unique arrow $\mathrm{m}_{\mathrm{Z}}: \mathrm{H}^{1} \times_{\mathrm{s}_{\mathrm{H}}, \mathrm{H}^{0}, \alpha} \mathrm{Z} \rightarrow \mathrm{Z}$ such that $\mathrm{m}_{\mathrm{Z}} \circ\left(\mathrm{pr}_{1} ; \mathrm{p} \circ \mathrm{pr}_{2}\right)=\tilde{\mathrm{r}}$. Elementwise $h \cdot\left[l_{0}\right]=\tilde{\mathrm{r}}\left(h ; l_{0}\right)$ for all $h \in \mathrm{H}^{1}, l_{0} \in \mathrm{L}^{0}$ with $\mathrm{s}_{\mathrm{H}}(h)=\mathrm{F}^{0}\left(l_{0}\right)$. For any element $l$ in $\mathrm{L}^{1}$ we have $\mathrm{F}^{1}(l) \cdot\left[\mathrm{s}_{\mathrm{L}}(l)\right]=\left[\mathrm{r}_{\mathrm{L}}(l)\right]$. We are going to show that $\left(\mathrm{Z} ; \mathrm{m}_{\mathrm{Z}} ; \alpha\right)$ is a left $\mathrm{H}$-action.

Since the arrows $\left(\mathrm{pr}_{1} ; \mathrm{p} \circ \mathrm{pr}_{2}\right)$ and $\left(\mathrm{F}^{1} ; \mathrm{s}_{\mathrm{L}}\right)$ are covers, they are coequalisers and therefore, they are epics. It is clear that the composition of epics is epic. So $\left(\mathrm{pr}_{1} ; \mathrm{p} \circ \mathrm{pr}_{2}\right) \circ\left(\mathrm{F}^{1} ; \mathrm{s}_{\mathrm{L}}\right)$ is epic. We also have

$$
\begin{aligned}
\alpha\left(\mathrm{F}^{1}(l) \cdot\left[\mathrm{s}_{\mathrm{L}}(l)\right]\right) & =\alpha\left(\left[\mathrm{r}_{\mathrm{L}}(l)\right]\right) \\
& =\mathrm{F}^{0}\left(\mathrm{r}_{\mathrm{L}}(l)\right) \\
& =\mathrm{r}_{\mathrm{H}}\left(\mathrm{F}^{1}(l)\right)
\end{aligned}
$$

for all $l \in \mathrm{L}^{1}$. Therefore, $\alpha \circ \mathrm{m}_{\mathrm{Z}} \circ\left(\mathrm{pr}_{1} ; \mathrm{p} \circ \mathrm{pr}_{2}\right) \circ\left(\mathrm{F}^{1} ; \mathrm{s}_{\mathrm{L}}\right)=\mathrm{r}_{\mathrm{H}} \circ \mathrm{pr}_{1} \circ\left(\mathrm{pr}_{1} ; \mathrm{p} \circ \mathrm{pr}_{2}\right) \circ\left(\mathrm{F}^{1} ; \mathrm{s}_{\mathrm{L}}\right)$. Thus $\alpha \circ \mathrm{m}_{\mathrm{Z}}=\mathrm{r}_{\mathrm{H}} \circ \mathrm{pr}_{1}$. This is the condition (1)

The next goal is to show that the arrow $\mathrm{m}_{\mathrm{Z}}: \mathrm{H}^{1} \times_{\mathrm{s}_{\mathrm{H}}, \mathrm{H}^{0}, \alpha} \mathrm{Z} \rightarrow \mathrm{Z}$ commutes with the multiplication map of $\mathrm{H}$.

Consider the following diagram

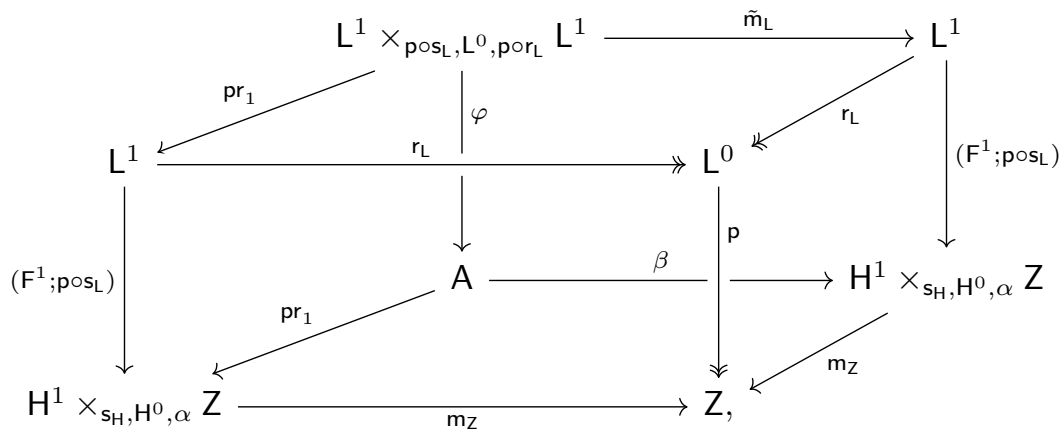

where

$$
\begin{gathered}
\mathrm{A}=\left(\mathrm{H}^{1} \times_{\mathrm{sH}_{\mathrm{H}}, \mathrm{H}^{0}, \alpha} \mathrm{Z}\right) \times_{\mathrm{pr}_{2}, \mathrm{Z}, \mathrm{m}_{\mathrm{Z}}}\left(\mathrm{H}^{1} \times_{\mathrm{sH}_{\mathrm{H}} \mathrm{H}^{0}, \alpha} \mathrm{Z}\right), \\
\beta=\left(\mathrm{m}_{\mathrm{H}} \circ\left(\mathrm{pr}_{1} \circ \mathrm{pr}_{1} ; \mathrm{pr}_{1} \circ \mathrm{pr}_{2}\right) ; \mathrm{pr}_{2} \circ \mathrm{pr}_{2}\right), \quad\left((h ; z) ;\left(h_{1} ; z_{1}\right)\right) \mapsto\left(h \cdot h_{1} ; z_{1}\right),
\end{gathered}
$$




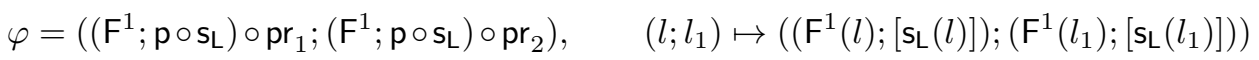
and $\tilde{\mathrm{m}}_{\mathrm{L}}=\mathrm{m}_{\mathrm{L}} \circ\left(\mathrm{pr}_{1} ; \mathrm{m}_{\mathrm{L}} \circ\left(\mathrm{pr}_{1} \circ \psi ; \mathrm{pr}_{2}\right)\right)$, where $\psi$ is the following composition:

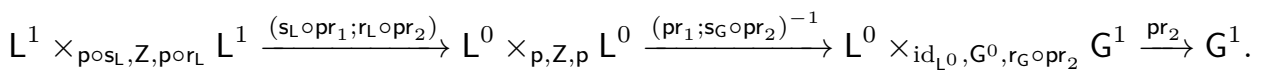

The arrow $\tilde{\mathrm{m}}_{\mathrm{L}}$ is defined elementwise by $\tilde{\mathrm{m}}_{\mathrm{L}}\left(l ; l_{1}\right)=l \cdot g \cdot l_{1}$ for all $l, l_{1} \in \mathrm{L}^{1}$ with $\left[\mathrm{s}_{\mathrm{L}}(l)\right]=\left[\mathrm{r}_{\mathrm{L}}\left(l_{1}\right)\right]$, where $g$ is the unique element in $\mathrm{G}^{1}$ such that $\mathrm{s}_{\mathrm{L}}(l) \cdot g=\mathrm{r}_{\mathrm{L}}\left(l_{1}\right)$. Since $\mathrm{pr}_{2}: \mathrm{H}^{1} \times_{\mathrm{s}_{\mathrm{H}}, \mathrm{H}^{0}, \alpha} \mathrm{Z} \rightarrow \mathrm{Z}$ is a pull-back of $\mathrm{s}_{\mathrm{H}}$, it is a cover, and therefore, the object $\mathrm{A}$ exists. Since $\mathrm{s}_{\mathrm{H}} \circ \mathrm{F}^{1}=\mathrm{F}^{0} \circ \mathrm{S}_{\mathrm{L}}=\alpha \circ \mathrm{p} \circ \mathrm{s}_{\mathrm{L}}$, the arrow $\left(\mathrm{F}^{1} ; \mathrm{p} \circ \mathrm{s}_{\mathrm{L}}\right)$ is well-defined. The arrow $\beta$ is well-defined because

$$
\begin{aligned}
\mathrm{s}_{\mathrm{H}}\left(h \cdot h_{1}\right) & =\mathrm{s}_{\mathrm{H}}\left(h_{1}\right) \\
& =\alpha\left(z_{1}\right)
\end{aligned}
$$

for all $h, h_{1} \in \mathrm{H}^{1}, z_{1} \in \mathrm{Z}$ with $\mathrm{s}_{\mathrm{H}}\left(h_{1}\right)=\alpha\left(z_{1}\right)$. The arrow $\varphi$ is well-defined because

$$
\begin{aligned}
\mathrm{F}^{1}\left(l_{1}\right) \cdot\left[\mathrm{s}_{\mathrm{L}}\left(l_{1}\right)\right] & =\left[\mathrm{r}_{\mathrm{L}}\left(l_{1}\right)\right] \\
& =\left[\mathrm{s}_{\mathrm{L}}(l)\right]
\end{aligned}
$$

for all $l, l_{1} \in \mathrm{L}^{1}$ with $\left[\mathrm{s}_{\mathrm{L}}(l)\right]=\left[\mathrm{r}_{\mathrm{L}}\left(l_{1}\right)\right]$. We are going to prove that each square commutes in the diagram above.

Since $\mathrm{r}_{\mathrm{L}}\left(l \cdot g \cdot l_{1}\right)=\mathrm{r}_{\mathrm{L}}(l)$ forall $l, l_{1} \in \mathrm{L}^{1}$ with $\left[\mathrm{s}_{\mathrm{L}}(l)\right]=\left[\mathrm{r}_{\mathrm{L}}\left(l_{1}\right)\right]$, the upper square commutes. We also have

$$
\begin{aligned}
\left(\mathrm{F}^{1}\left(l \cdot g \cdot l_{1}\right) ;\left[\mathrm{s}_{\mathrm{L}}\left(l \cdot g \cdot l_{1}\right)\right]\right) & =\left(\mathrm{F}^{1}(l) \cdot \mathrm{F}^{1}(g) \cdot \mathrm{F}^{1}\left(l_{1}\right) ;\left[\mathrm{s}_{\mathrm{L}}\left(l_{1}\right)\right]\right) \\
& =\left(\mathrm{F}^{1}(l) \cdot \mathrm{F}^{1}\left(l_{1}\right) ;\left[\mathrm{s}_{\mathrm{L}}\left(l_{1}\right)\right]\right) \\
& =\beta\left(\left(\mathrm{F}^{1}(l) ;\left[\mathrm{s}_{\mathrm{L}}(l)\right]\right) ;\left(\mathrm{F}^{1}\left(l_{1}\right) ;\left[\mathrm{s}_{\mathrm{L}}\left(l_{1}\right)\right]\right)\right) \\
& =\beta\left(\varphi\left(l ; l_{1}\right)\right)
\end{aligned}
$$

for all $l, l_{1} \in \mathrm{L}^{1}$ with $\left[\mathrm{s}_{\mathrm{L}}(l)\right]=\left[\mathrm{r}_{\mathrm{L}}\left(l_{1}\right)\right]$. Therefore, $\beta \circ \varphi=\left(\mathrm{F}^{1} ; \mathrm{p} \circ \mathrm{s}_{\mathrm{L}}\right) \circ \tilde{\mathrm{m}}_{\mathrm{L}}$. The left and right squares and the square face to the reader commute obviously. Finally, We have

$$
\begin{aligned}
\mathrm{m}_{\mathrm{Z}} \circ \mathrm{pr}_{1} \circ \varphi & =\mathrm{m}_{\mathrm{Z}} \circ\left(\mathrm{F}^{1} ; \mathrm{p} \circ \mathrm{s}_{\mathrm{L}}\right) \circ \mathrm{pr}_{1} \\
& =\mathrm{p} \circ \mathrm{r}_{\mathrm{L}} \circ \mathrm{pr}_{1} \\
& =\mathrm{p} \circ \mathrm{r}_{\mathrm{L}} \circ \mathrm{m}_{\mathrm{L}} \\
& =\mathrm{m}_{\mathrm{Z}} \circ\left(\mathrm{F}^{1} ; \mathrm{p} \circ \mathrm{s}_{\mathrm{L}}\right) \circ \mathrm{m}_{\mathrm{L}} \\
& =\mathrm{m}_{\mathrm{Z}} \circ \beta \circ \varphi .
\end{aligned}
$$

Hence $\mathrm{m}_{\mathrm{Z}} \circ \mathrm{pr}_{1} \circ \varphi=\mathrm{m}_{\mathrm{Z}} \circ \beta \circ \varphi$. The next goal is to show that the arrow $\varphi$ is epic. Consider the following diagram:

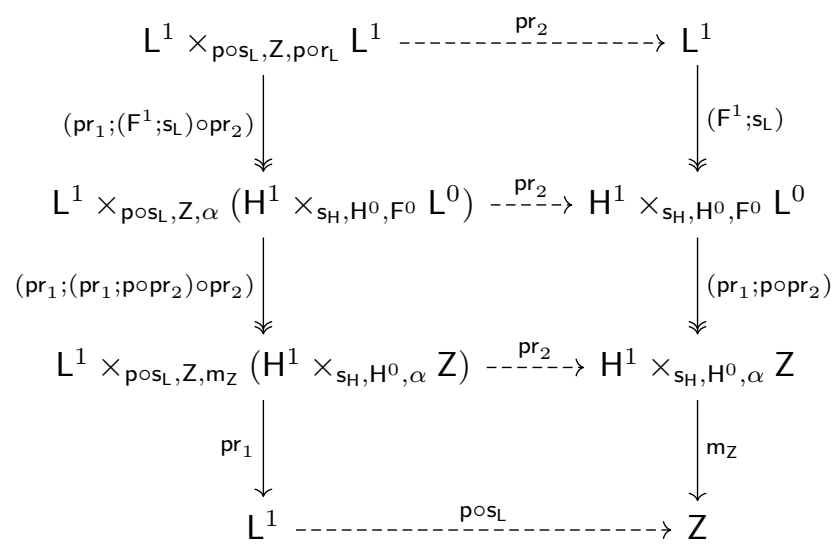


Each square is a pull-back square in it. Under Assumption 2.15 the arrows $\left(\mathrm{pr}_{1} ;\left(\mathrm{F}^{1} ; \mathrm{s}_{\mathrm{L}}\right) \circ \mathrm{pr}_{2}\right)$ and $\left(\mathrm{pr}_{1} ;\left(\mathrm{pr}_{1} ; \mathrm{p} \circ \mathrm{pr}_{2}\right) \circ \mathrm{pr}_{2}\right)$ are covers. Hence they are coequalisers and therefore, epics. Thus their composition

$$
\left(\mathrm{pr}_{1} ;\left(\mathrm{pr}_{1} ; \mathrm{p} \circ \mathrm{pr}_{2}\right) \circ \mathrm{pr}_{2}\right) \circ\left(\mathrm{pr}_{1} ;\left(\mathrm{F}^{1} ; \mathrm{s}_{\mathrm{L}}\right) \circ \mathrm{pr}_{2}\right)=\left(\mathrm{pr}_{1} ;\left(\mathrm{F}^{1} ; \mathrm{p} \circ \mathrm{s}_{\mathrm{L}}\right) \circ \mathrm{pr}_{2}\right)
$$

is epic. Analogously, if we consider the pull-backs of the following sequence of the composable covers

$$
\mathrm{L}^{1} \stackrel{\left(\mathrm{F}^{1} ; \mathrm{sL}_{\mathrm{L}}\right)}{\longrightarrow} \mathrm{H}^{1} \times_{\mathrm{s}_{\mathrm{H}}, \mathrm{H}^{0}, \mathrm{~F}^{0}} \quad \mathrm{~L}^{0} \stackrel{\left(\left(\mathrm{pr}_{1} ; \mathrm{popr}_{2}\right) \mathrm{opr}_{1} ; \mathrm{pr}_{2}\right)}{\longrightarrow} \mathrm{H}^{1} \times_{\mathrm{SH}_{\mathrm{H}}, \mathrm{H}^{0}, \alpha} \quad \mathrm{Z} \stackrel{\mathrm{pr}_{2}}{\longrightarrow \mathrm{Z}}
$$

along the arrow $\mathrm{m}_{\mathrm{Z}}: \mathrm{H}^{1} \times_{\mathrm{SH}_{\mathrm{H}} \mathrm{H}^{0}, \alpha} \mathrm{Z} \rightarrow \mathrm{Z}$, we get that the following composition

$$
\left(\left(\mathrm{pr}_{1} ; \mathrm{p} \circ \mathrm{pr}_{2}\right) \circ \mathrm{pr}_{1} ; \mathrm{pr}_{2}\right) \circ\left(\left(\mathrm{F}^{1} ; \mathrm{sL}_{\mathrm{L}}\right) \circ \mathrm{pr}_{1} ; \mathrm{pr}_{2}\right)=\left(\left(\mathrm{F}^{1} ; \mathrm{p} \circ \mathrm{s}_{\mathrm{L}}\right) \circ \mathrm{pr}_{1} ; \mathrm{pr}_{2}\right)
$$

is epic. Finally,

$$
\begin{aligned}
& \left(\left(\mathrm{F}^{1} ; \mathrm{p} \circ \mathrm{s}_{\mathrm{L}}\right) \circ \mathrm{pr}_{1} ; \mathrm{pr}_{2}\right) \circ\left(\mathrm{pr}_{1} ;\left(\mathrm{F}^{1} ; \mathrm{p} \circ \mathrm{s}_{\mathrm{L}}\right) \circ \mathrm{pr}_{2}\right) \\
= & \left(\left(\mathrm{F}^{1} ; \mathrm{p} \circ \mathrm{s}_{\mathrm{L}}\right) \circ \mathrm{pr}_{1} ;\left(\mathrm{F}^{1} ; \mathrm{p} \circ \mathrm{s}_{\mathrm{L}}\right) \circ \mathrm{pr}_{2}\right) \\
= & \varphi
\end{aligned}
$$

is epic. Therefore, $\mathrm{m}_{\mathrm{Z}} \circ \beta=\mathrm{m}_{\mathrm{Z}} \circ \mathrm{pr}_{1}$. Elementwise $\left(h \cdot h_{1}\right) \cdot z_{1}=h \cdot z$ for all $\left((h ; z) ;\left(h_{1} ; z_{1}\right)\right) \in$ A. Since $z=h_{1} \cdot z_{1}$, we have $\left(h \cdot h_{1}\right) \cdot z_{1}=h \cdot\left(h_{1} \cdot z_{1}\right)$ for all $h, h_{1} \in \mathrm{H}^{1}, z_{1} \in \mathrm{Z}$ with $\mathrm{s}_{\mathbf{H}}(h)=\mathrm{r}_{\mathbf{H}}\left(h_{1}\right)$ and $\mathrm{s}_{\mathbf{H}}\left(h_{1}\right)=\alpha\left(z_{1}\right)$. So the condition (2) holds.

We also have

$$
\begin{aligned}
1_{\alpha\left(\left[l_{0}\right]\right)} \cdot\left[l_{0}\right] & =\tilde{\mathrm{r}}\left(1_{\alpha\left(\left[l_{0}\right]\right)} ;\left[l_{0}\right]\right) \\
& =\tilde{\mathrm{r}}\left(1_{\mathrm{F}^{0}\left(l_{0}\right)} ; l_{0}\right) \\
& =\tilde{\mathrm{r}}\left(\mathrm{F}^{1}\left(1_{\left(l_{0}\right)}\right) ; l_{0}\right) \\
& =\tilde{\mathrm{r}}\left(\mathrm{F}^{1}\left(1_{\left(l_{0}\right)}\right) ; \mathrm{s}_{\mathrm{L}}\left(1_{\left(l_{0}\right)}\right)\right) \\
& =\left[\mathrm{r}_{\mathrm{L}}\left(1_{\left(l_{0}\right)}\right)\right] \\
& =\left[l_{0}\right]
\end{aligned}
$$

for all $l_{0} \in \mathrm{L}^{0}$. Since $\mathrm{p}: \mathrm{L}^{0} \rightarrow \mathrm{Z}$ is a cover, it is epic and therefore, $\mathrm{m}_{\mathrm{Z}} \circ\left(\mathrm{u}_{\mathrm{H}} \circ \alpha ; \mathrm{id}_{\mathrm{Z}}\right)=$ $\mathrm{id}_{\mathrm{Z}}$. So we can deduce that $\left(\mathrm{Z} ; \mathrm{m}_{\mathrm{Z}} ; \alpha\right)$ is a left $\mathrm{H}$-action by Remark 5.11 .

Let the groupoid $L / G$ be the transformation groupoid of the left $\mathrm{H}$-action $\left(\mathrm{Z} ; \mathrm{m}_{\mathrm{Z}} ; \alpha\right)$. We know from Example 5.31 that there is a groupoid covering $\mathrm{F}_{2}: \mathrm{H} \ltimes \mathrm{Z} \rightarrow$ $\mathrm{H}$ where $\mathrm{F}_{2}^{1}=\mathrm{pr}_{1}: \mathrm{H}^{1} \times_{\mathrm{s}_{\mathrm{H}}, \mathrm{H}^{0}, \alpha} \mathrm{Z} \rightarrow \mathrm{H}^{1}$ and $\mathrm{F}_{2}^{0}=\alpha: \mathrm{Z} \rightarrow \mathrm{H}^{0}$.

There is a functor $\mathrm{F}_{1}: \mathrm{L} \rightarrow \mathrm{H} \ltimes \mathrm{Z}$, where $\mathrm{F}_{1}^{1}=\left(\mathrm{F}^{1} ; \mathrm{p} \circ \mathrm{s}_{\mathrm{L}}\right): \mathrm{L}^{1} \rightarrow \mathrm{H}^{1} \times_{\mathrm{sH}_{\mathrm{H}} \mathrm{H}^{0}, \alpha} \mathrm{Z}$ and $F_{1}^{0}=p: L^{0} \rightarrow Z$. These arrows intertwine the range maps because the right square commutes in the diagram above. They intertwine the source maps because $\mathrm{pr}_{2} \circ\left(\mathrm{F}^{1} ; \mathrm{p} \circ \mathrm{s}_{\mathrm{L}}\right)=\mathrm{p} \circ \mathrm{s}_{\mathrm{L}}$. Notice that the multiplication map of $\mathrm{H} \ltimes \mathrm{Z}$ is $\beta$. We have

$$
\begin{aligned}
\left(\mathrm{F}^{1} ; \mathrm{p} \circ \mathrm{s}_{\mathrm{L}}\right)\left(l \cdot l_{1}\right) & =\left(\mathrm{F}^{1}\left(l \cdot l_{1}\right) ;\left[\mathrm{s}_{\mathrm{L}}\left(l \cdot l_{1}\right)\right]\right) \\
& =\left(\mathrm{F}^{1}(l) \cdot \mathrm{F}^{1}\left(l_{1}\right) ;\left[\mathrm{s}_{\mathrm{L}}\left(l_{1}\right)\right]\right) \\
& =\left(\mathrm{F}^{1}(l) ;\left[\mathrm{s}_{\mathrm{L}}(l)\right]\right) \cdot\left(\mathrm{F}^{1}\left(l_{1}\right) ;\left[\mathrm{s}_{\mathrm{L}}\left(l_{1}\right)\right]\right) \\
& =\left(\mathrm{F}^{1} ; \mathrm{p} \circ \mathrm{s}_{\mathrm{L}}\right)(l) \cdot\left(\mathrm{F}^{1} ; \mathrm{p} \circ \mathrm{s}_{\mathrm{L}}\right)\left(l_{1}\right)
\end{aligned}
$$

for all $l, l_{1} \in \mathrm{L}^{1}$ with $\mathrm{s}_{\mathrm{L}}(l)=\mathrm{r}_{\mathrm{L}}\left(l_{1}\right)$. Therefore, $\mathrm{F}_{1}: \mathrm{L} \rightarrow \mathrm{H} \ltimes \mathrm{Z}$ intertwines the multiplication maps, too. We need to check that this functor is a groupoid fibration. There is a canonical isomorphism

$$
\left(\mathrm{H}^{1} \times_{\mathrm{s}_{\mathrm{H}}, \mathrm{H}^{0}, \alpha} \mathrm{Z}\right) \times_{\mathrm{pr}_{2}, \mathrm{Z}, \mathrm{p}} \mathrm{L}^{0} \stackrel{\left(\mathrm{pr}_{1} \mathrm{opr}_{1} ; \mathrm{pr}_{2}\right)}{\longrightarrow} \mathrm{H}^{1} \times_{\mathrm{s}_{\mathrm{H}}, \mathrm{H}^{0}, \mathrm{~F}^{0}} \mathrm{~L}^{0} .
$$




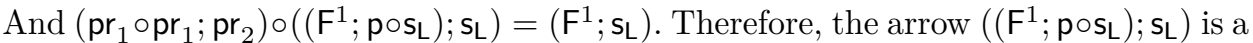
cover, which shows that $F_{1}: L \rightarrow H \ltimes Z$ is a groupoid fibration. Since $\operatorname{pr}_{1} \circ\left(F^{1} ; s_{L}\right)=F^{1}$ and $\alpha \circ \mathrm{p}=\mathrm{F}^{0}$, the following diagram commutes

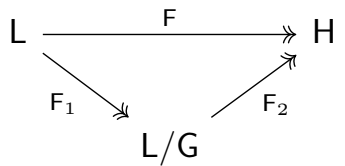

Finally, the fibre of $\mathrm{F}_{1}: \mathrm{L} \rightarrow \mathrm{L} / \mathrm{G}$ is $\mathrm{G}$ by Corollary 4.23

Proposition 5.69. Let $\mathrm{F}: \mathrm{L} \rightarrow \mathrm{H}$ be a groupoid fibration with fibre $\mathrm{G}$. Under Assumptions 5.63, 2.15 and 2.14, if the groupoids $\mathrm{G}$ and $\mathrm{H}$ are basic, then so is $\mathrm{L}$.

Proof. Since the groupoid $\mathrm{H}$ is basic, the left $\mathrm{H}$-action $\left(\mathrm{Z} ; \mathrm{m}_{\mathrm{Z}} ; \alpha\right)$, used in the proof of Proposition 5.68 is basic by Assumption 5.63. Therefore, the quotient groupoid $\mathrm{L} / \mathrm{G}$ is basic by Corollary 5.62 in the global case. That is, there are an object $\mathrm{Q}$ and a cover $\mathrm{q}: \mathrm{Z} \rightarrow \mathrm{Q}$ such that the arrow

$$
\mathrm{H}^{1} \times_{\mathrm{sH}_{\mathrm{H}}, \mathrm{H}^{0}, \alpha} \mathrm{Z} \stackrel{\left(\mathrm{pr}_{2} ; \mathrm{m}_{\mathrm{Z}}\right)}{\longrightarrow} \mathrm{Z} \times_{\mathrm{q}, \mathrm{Q}, \mathrm{q}} \mathrm{Z}, \quad(h ; z) \mapsto(h ; h \cdot z),
$$

is a well-defined isomorphism. We are going to show that the arrow

$$
\mathrm{L}^{1} \stackrel{\left(\mathrm{s}_{\mathrm{L}} ; \mathrm{r}_{\mathrm{L}}\right)}{\longrightarrow} \mathrm{L}^{0} \times_{\mathrm{qop}, \mathrm{Q}, \mathrm{q} \circ \mathrm{p}} \mathrm{L}^{0} \quad l \mapsto\left(\mathrm{s}_{\mathrm{L}}(l) ; \mathrm{r}_{\mathrm{L}}(l)\right)
$$

is a well-defined isomorphism, which gives that the groupoid $L$ is basic. It is well-defined because

$$
\begin{aligned}
{\left[\left[\mathrm{r}_{\mathrm{L}}(l)\right]\right] } & =\left[\mathrm{F}^{1}(l) \cdot\left[\mathrm{s}_{\mathrm{L}}(l)\right]\right] \\
& =\left[\left[\mathrm{s}_{\mathrm{L}}(l)\right]\right]
\end{aligned}
$$

for all $l \in \mathrm{L}^{1}$.

Consider the pull-back of the G-bundle $\left(\mathrm{L}^{0} ; \mathrm{s}_{\mathrm{G}} \circ \mathrm{pr}_{2} ; \mathrm{id}_{\mathrm{G}^{0}}\right)$ over $\mathrm{q} \circ \mathrm{p}: \mathrm{L}^{0} \rightarrow \mathrm{Q}$ along $\mathrm{q} \circ \mathrm{p}: \mathrm{L}^{0} \rightarrow \mathrm{Q}$. That is $\left(\mathrm{L}^{0} \times_{\mathrm{qop}, \mathrm{Q}, \mathrm{q} \circ \mathrm{p}} \mathrm{L}^{0} ; \mathrm{m}_{1} ; \mathrm{pr}_{2}\right)$, where $\mathrm{m}_{1}=\left(\mathrm{pr}_{1} \circ \mathrm{pr}_{1} ; \mathrm{s}_{\mathrm{G}} \circ \mathrm{pr}_{2}\right)$. Elementwise $\left(l_{0} ; l_{0}^{\prime}\right) \cdot g=\left(l_{0} ; l_{0}^{\prime} \cdot g\right)$ for all $l_{0}, l_{0}^{\prime} \in \mathrm{L}^{0}, g \in \mathrm{G}^{1}$ with $\left[\left[l_{0}\right]\right]=\left[\left[l_{0}^{\prime}\right]\right]$ and $l_{0}^{\prime}=\mathrm{r}_{\mathrm{G}}(g)$. Since the G-bundle $\left(\mathrm{L}^{0} ; \mathrm{s}_{\mathrm{G}} \circ \mathrm{pr}_{2} ; \mathrm{id}_{\mathrm{G}^{0}}\right)$ over $\mathrm{q} \circ \mathrm{p}: \mathrm{L}^{0} \rightarrow \mathrm{Q}$ is a principal G-bundle over $p: L^{0} \rightarrow Z$, its pull-back along the arrow $q \circ p: L^{0} \rightarrow Q$ is a principal $G$ bundle over $\left(\mathrm{pr}_{1} ; \mathrm{p}\right.$ o $\left.\mathrm{pr}_{2}\right): \mathrm{L}^{0} \times_{\mathrm{qop}, \mathrm{Q}, \mathrm{qop}} \mathrm{L}^{0} \rightarrow \mathrm{L}^{0} \times_{\mathrm{q} o \mathrm{p}, \mathrm{Q}, \mathrm{q}} \mathrm{Z}$ by Lemma 5.50 Also, there

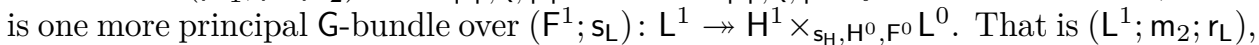
where $\mathrm{m}_{2}=\mathrm{m}_{\mathrm{L}} \circ\left(\mathrm{pr}_{1} ; \mathrm{i}_{\mathrm{L}} \circ \mathrm{pr}_{1} \circ \mathrm{pr}_{2}\right)$. Elementwise $l \cdot g=g^{-1} \cdot l$ for all $l \in \mathrm{L}^{1}, g \in \mathrm{G}^{1}$ with $\mathrm{r}_{\mathrm{L}}(l)=\mathrm{r}_{\mathrm{G}}(g)$. We are going to show that the arrow $\left(\mathrm{s}_{\mathrm{L}} ; \mathrm{r}_{\mathrm{L}}\right): \mathrm{L}^{1} \rightarrow \mathrm{L}^{0} \times_{\mathrm{qop}, \mathrm{Q}, \mathrm{qop}} \mathrm{L}^{0}$

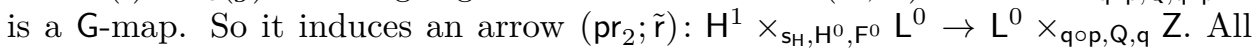
needed arrows are in the following diagram:

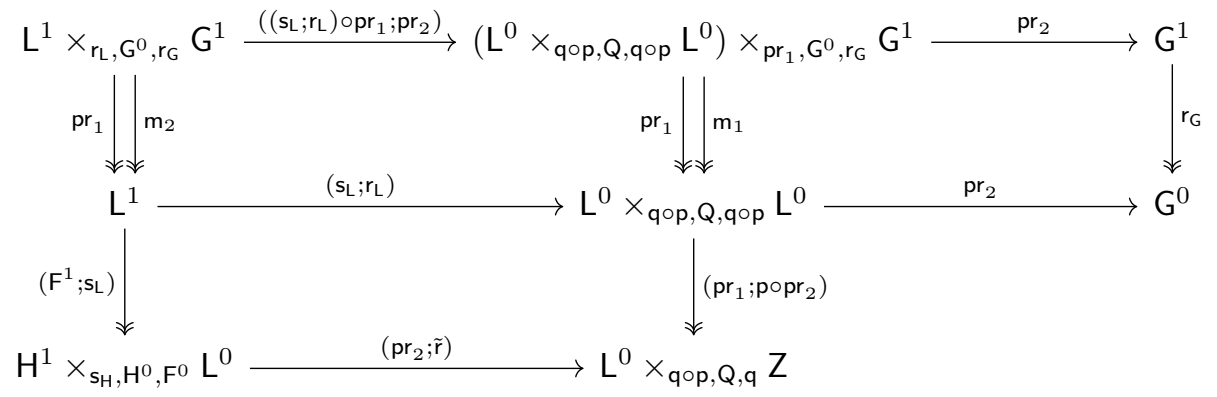


The arrow $\left(s_{\mathrm{L}} ; r_{\mathrm{L}}\right): \mathrm{L}^{1} \rightarrow \mathrm{L}^{0} \times_{\mathrm{qop}, \mathrm{Q}, \mathrm{qop}} \mathrm{L}^{0}$ commutes with the anchor maps, that is $\mathrm{pr}_{2} \circ\left(\mathrm{s}_{\mathrm{L}} ; \mathrm{r}_{\mathrm{L}}\right)=\mathrm{r}_{\mathrm{L}}$. It also commutes with the action maps because

$$
\begin{aligned}
\left(\mathrm{s}_{\mathrm{L}} ; \mathrm{r}_{\mathrm{L}}\right)(l \cdot g) & =\left(\mathrm{s}_{\mathrm{L}} ; r_{\mathrm{L}}\right)\left(g^{-1} \cdot l\right) \\
& =\left(\mathrm{s}_{\mathrm{L}}\left(g^{-1} \cdot l\right) ; \mathrm{r}_{\mathrm{L}}\left(g^{-1} \cdot l\right)\right) \\
& =\left(\mathrm{s}_{\mathrm{L}}(l) ; \mathrm{r}_{\mathrm{L}}\left(g^{-1}\right)\right) \\
& =\left(\mathrm{s}_{\mathrm{L}}(l) ; \mathrm{s}_{\mathrm{L}}(g)\right) \\
& =\left(\mathrm{s}_{\mathrm{L}}(l) ; \mathrm{r}_{\mathrm{L}}(g) \cdot g\right) \\
& =\left(\mathrm{s}_{\mathrm{L}}(l) ; \mathrm{r}_{\mathrm{L}}(l) \cdot g\right) \\
& =\left(\mathrm{s}_{\mathrm{L}}(l) ; \mathrm{r}_{\mathrm{L}}(l)\right) \cdot g \\
& =\left(\mathrm{s}_{\mathrm{L}} ; \mathrm{r}_{\mathrm{L}}\right)(l) \cdot g
\end{aligned}
$$

for all $l \in \mathrm{L}^{1}, g \in \mathrm{G}^{1}$ with $\mathrm{r}_{\mathrm{L}}(l)=\mathrm{r}_{\mathrm{L}}(g)$. So the arrow $\left(\mathrm{s}_{\mathrm{L}} ; \mathrm{r}_{\mathrm{L}}\right): \mathrm{L}^{1} \rightarrow \mathrm{L}^{0} \times_{\mathrm{qop}, \mathrm{Q}, \mathrm{qop}} \mathrm{L}^{0}$ is a $\mathrm{G}$-map. Also, the bottom square commutes because

$$
\begin{aligned}
\left(\mathrm{pr}_{2} ; \tilde{\mathrm{r}}\right)\left(\left(\mathrm{F}^{1} ; \mathrm{s}_{\mathrm{L}}\right)(l)\right) & =\left(\mathrm{pr}_{2} ; \tilde{\mathrm{r}}\right)\left(\mathrm{F}^{1}(l) ; \mathrm{s}_{\mathrm{L}}(l)\right) \\
& =\left(\mathrm{s}_{\mathrm{L}}(l) ; \tilde{\mathrm{r}}\left(\mathrm{F}^{1}(l) ; \mathrm{s}_{\mathrm{L}}(l)\right)\right) \\
& =\left(\mathrm{s}_{\mathrm{L}}(l) ;\left[\mathrm{r}_{\mathrm{L}}(l)\right]\right) \\
& =\left(\mathrm{pr}_{1} ; \mathrm{p} \circ \mathrm{pr}_{2}\right)\left(\mathrm{s}_{\mathrm{L}}(l) ; \mathrm{r}_{\mathrm{L}}(l)\right) \\
& =\left(\mathrm{pr}_{1} ; \mathrm{p} \circ \mathrm{pr}_{2}\right)\left(\left(\mathrm{s}_{\mathrm{L}} ; \mathrm{r}_{\mathrm{L}}\right)(l)\right)
\end{aligned}
$$

for all $l \in \mathrm{L}^{1}$. Therefore, the bottom square is a pull-back square by Proposition 5.48 . Hence $\left(\mathrm{s}_{\mathrm{L}} ; \mathrm{r}_{\mathrm{L}}\right)$ induces $\left(\mathrm{pr}_{2} ; \tilde{\mathrm{r}}\right)$. Hence one of them is an isomorphism if and only if the other one is by Corollary 5.54 .

Consider the pull-back of the G-bundle $\left(\mathrm{L}^{0} ; \mathrm{s}_{\mathrm{G}} \circ \mathrm{pr}_{2} ; \mathrm{id}_{\mathrm{G}^{0}}\right)$ over $\mathrm{q} \circ \mathrm{p}: \mathrm{L}^{0} \rightarrow \mathrm{Q}$ along $\mathrm{q}: \mathrm{Z} \rightarrow \mathrm{Q}$. That is $\left(\mathrm{Z} \times_{\mathrm{q}, \mathrm{Q}, \mathrm{q} \circ \mathrm{p}} \mathrm{L}^{0} ; \tilde{\mathrm{m}}_{1} ; \mathrm{pr}_{2}\right)$, where $\tilde{\mathrm{m}}_{1}=\left(\mathrm{pr}_{1} \circ \mathrm{pr}_{1} ; \mathrm{s}_{\mathrm{G}} \circ \mathrm{pr}_{2}\right)$. Elementwise $\left(z ; l_{0}\right) \cdot g=\left(z ; l_{0} \cdot g\right)$ for all $z \in \mathrm{Z}, l_{0} \in \mathrm{L}^{0}, g \in \mathrm{G}^{1}$, with $[z]=\left[\left[l_{0}\right]\right]$ and $l_{0}=\mathrm{r}_{\mathrm{G}}(g)$. Analogously, we can say that this action is a principal G-bundle over the arrow $\left(\mathrm{pr}_{1} ; \mathrm{p} \circ \mathrm{pr}_{2}\right): \mathrm{Z} \times_{\mathrm{q}, \mathrm{Q}, \mathrm{q} \circ \mathrm{p}} \mathrm{L}^{0} \rightarrow \mathrm{Z} \times_{\mathrm{q}, \mathrm{Q}, \mathrm{q}} \mathrm{Z}$ by Lemma 5.50 Also, we have one more G-

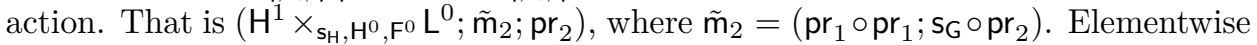
$\left(h ; l_{0}\right) \cdot g=\left(h ; l_{0} \cdot g\right)$ for all $h \in \mathrm{H}^{1}, l_{0} \in \mathrm{L}^{0}, g \in \mathrm{G}^{1}$ with $\mathrm{s}_{\mathrm{H}}(h)=\mathrm{F}^{0}\left(l_{0}\right)$ and $l_{0}=\mathrm{r}_{\mathrm{G}}(g)$.

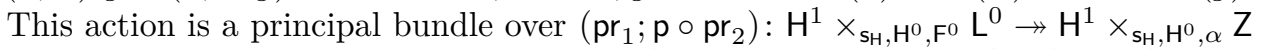
because it is a pull-back of a G-bundle $\left(\mathrm{L}^{0} ; \mathrm{s}_{\mathrm{G}} \circ \mathrm{pr}_{2} ; \mathrm{id}_{\mathrm{G}^{0}}\right)$ over $\mathrm{F}^{0}: \mathrm{L}^{0} \rightarrow \mathrm{H}^{0}$ along $s_{L}: H^{1} \rightarrow H^{0}$, which is principal over $p: L^{0} \rightarrow Z$. We are going to show that the

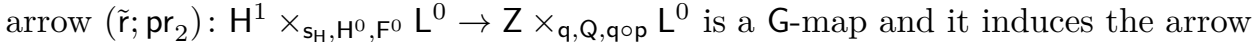
$\left(\mathrm{m}_{\mathrm{Z}} ; \mathrm{pr}_{2}\right): \mathrm{H}^{1} \times_{\mathrm{s}_{\mathrm{H}}, \mathrm{H}^{0}, \alpha} \mathrm{Z} \rightarrow \mathrm{Z} \times_{\mathrm{q}, \mathrm{Q}, \mathrm{q}} \mathrm{Z}$. All needed arrows are in the following diagram:

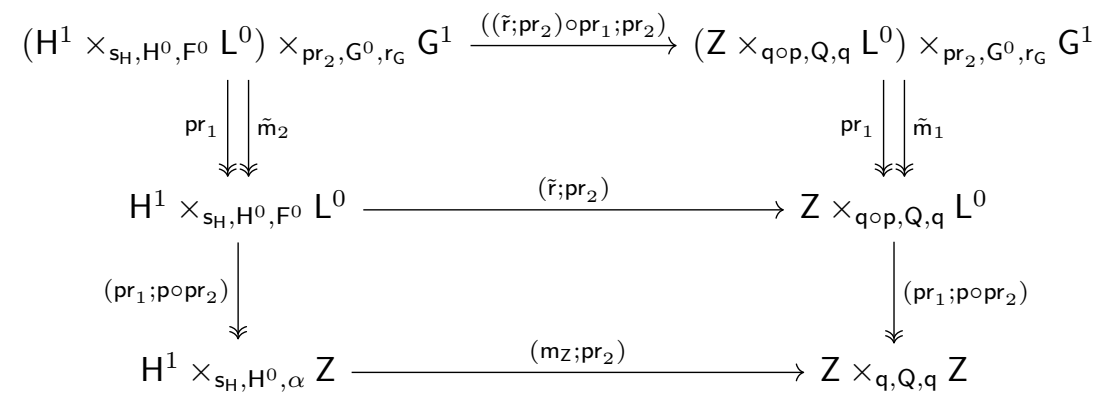


The arrow $\left(\tilde{\mathrm{r}} ; \mathrm{pr}_{2}\right)$ clearly commutes with the anchor maps, $\mathrm{pr}_{2} \circ\left(\tilde{\mathrm{r}} ; \mathrm{pr}_{2}\right)=\mathrm{pr}_{2}$. It also commutes with the action maps because

$$
\begin{aligned}
\left(\tilde{\mathrm{r}} ; \mathrm{pr}_{2}\right)\left(\left(h ; l_{0}\right) \cdot g\right) & =\left(\tilde{\mathrm{r}} ; \mathrm{pr}_{2}\right)\left(h ; l_{0} \cdot g\right) \\
& =\left(\tilde{\mathrm{r}}\left(h ; l_{0} \cdot g\right) ; l_{0} \cdot g\right) \\
& =\left(h \cdot\left[l_{0} \cdot g\right] ; l_{0} \cdot g\right) \\
& =\left(h \cdot\left[l_{0}\right] ; l_{0} \cdot g\right) \\
& =\left(\tilde{\mathrm{r}}\left(h ; l_{0}\right) ; l_{0} \cdot g\right) \\
& =\left(\tilde{\mathrm{r}}\left(h ; l_{0}\right) ; l_{0}\right) \cdot g \\
& =\left(\tilde{\mathrm{r}} ; \mathrm{pr}_{2}\right)\left(h ; l_{0}\right) \cdot g
\end{aligned}
$$

for all $h \in \mathrm{H}^{1}, l_{0} \in \mathrm{L}^{0}, g \in \mathrm{G}^{1}$ with $\mathrm{s}_{\mathrm{H}}(h)=\mathrm{F}^{0}\left(l_{0}\right)$ and $l_{0}=\mathrm{r}_{\mathrm{G}}(g)$. The bottom square commutes because

$$
\begin{aligned}
\left(\mathrm{pr}_{1} ; \mathrm{p} \circ \mathrm{pr}_{2}\right)\left(\left(\tilde{\mathrm{r}} ; \mathrm{pr}_{2}\right)\left(h ; l_{0}\right)\right) & =\left(\mathrm{pr}_{1} ; \mathrm{p} \circ \mathrm{pr}_{2}\right)\left(\tilde{\mathrm{r}}\left(h ; l_{0}\right) ; l_{0}\right) \\
& =\left(\mathrm{pr}_{1} ; \mathrm{p} \circ \mathrm{pr}_{2}\right)\left(h \cdot\left[l_{0}\right] ; l_{0}\right) \\
& =\left(h \cdot\left[l_{0}\right] ;\left[l_{0}\right]\right) \\
& =\left(\mathrm{m}_{\mathrm{Z}} ; \mathrm{pr}_{2}\right)\left(h ;\left[l_{0}\right]\right) \\
& =\left(\mathrm{m}_{\mathrm{Z}} ; \mathrm{pr}_{2}\right)\left(\left(\mathrm{pr}_{1} ; \mathrm{p} \circ \mathrm{pr}_{2}\right)\left(h ; l_{0}\right)\right)
\end{aligned}
$$

for all $h \in \mathrm{H}^{1}, l_{0} \in \mathrm{L}^{0}$ with $\mathrm{s}_{\mathrm{H}}(h)=\mathrm{F}^{0}\left(l_{0}\right)$. Therefore, the arrow $\left(\mathrm{m}_{\mathrm{Z}} ; \mathrm{pr}_{2}\right)$ induces $\left(\tilde{\mathrm{r}} ; \mathrm{pr}_{2}\right)$. Hence $\left(\tilde{\mathrm{r}} ; \mathrm{pr}_{2}\right)$ is an isomorphism by Corollary 5.54. Thus the arrow

$$
\left(\mathrm{s}_{\mathrm{L}} ; \mathrm{r}_{\mathrm{L}}\right): \mathrm{L}^{1} \rightarrow \mathrm{L}^{0} \times_{\mathrm{qop}, \mathrm{Q}, \mathrm{qop}} \mathrm{L}^{0}
$$

is an isomorphism for the same reason. So the groupoid $L$ is basic because the arrow $\mathrm{q} \circ \mathrm{p}$ is a cover by Assumption 2.14

\section{Generalised morphisms Between Groupoids}

There are several types of generalised morphisms between groupoids which form categories with groupoids as objects.

6.1. Actors. Let $\mathrm{G}=\left(\mathrm{G}^{0}, \mathrm{G}^{1}, \mathrm{r}_{\mathrm{G}}, \mathrm{s}_{\mathrm{G}}, \mathrm{m}_{\mathrm{G}}, \mathrm{u}_{\mathrm{G}}, \mathrm{i}_{\mathrm{G}}\right)$ and $\mathrm{H}=\left(\mathrm{H}^{0}, \mathrm{H}^{1}, \mathrm{r}_{\mathrm{H}}, \mathrm{s}_{\mathrm{H}}, \mathrm{m}_{\mathrm{H}}, \mathrm{u}_{\mathrm{H}}, \mathrm{i}_{\mathrm{H}}\right)$ be groupoids in a category $\left(\mathcal{C}, \mathcal{F}_{p}\right)$ with partial covers.

Definition 6.1. [47, Definition 4.15] An actor from $\mathrm{G}$ to $\mathrm{H}$ is a pair of arrows $(\mathrm{m} ; \mathrm{r})$ such that $\left(\mathrm{H}^{1} ; \mathrm{m} ; \mathrm{r}\right)$ is a left $\mathrm{G}$-action which commutes with the right translation action $\left(\mathrm{H}^{1} ; \mathrm{m}_{\mathrm{H}} ; \mathrm{s}_{\mathrm{H}}\right)$. That is,

(1) $\mathrm{s}_{\mathrm{H}} \circ \mathrm{m}=\mathrm{s}_{\mathrm{H}} \circ \mathrm{pr}_{2} \quad \mathrm{~s}_{\mathrm{H}}(g \cdot h)=\mathrm{s}_{\mathrm{H}}(h), \quad \forall g \in \mathrm{G}^{1}, \forall h \in \mathrm{H}^{1}$ with $\mathrm{s}_{\mathrm{G}}(g)=\mathrm{r}(h)$;

(2) $r \circ \mathrm{m}_{\mathrm{H}}=\mathrm{r} \circ \mathrm{pr}_{1} \quad \mathrm{r}\left(h \cdot h_{1}\right)=\mathrm{r}(h), \quad \forall h, h_{1} \in \mathrm{H}^{1}$ with $\mathrm{s}_{\mathrm{H}}(h)=\mathrm{r}_{\mathrm{H}}\left(h_{1}\right)$;

(3) $\mathrm{m}_{\mathrm{H}} \circ\left(\mathrm{m} \circ \mathrm{pr}_{1} ; \mathrm{pr}_{2} \circ \mathrm{pr}_{2}\right)=\mathrm{m} \circ\left(\mathrm{pr}_{1} \circ \mathrm{pr}_{1} ; \mathrm{m}_{\mathrm{H}} \circ \mathrm{pr}_{2}\right) \quad(g \cdot h) \cdot h_{1}=g \cdot\left(h \cdot h_{1}\right)$ for all $g \in \mathrm{G}^{1}, h, h_{1} \in \mathrm{H}^{1}$ with $\mathrm{s}_{\mathrm{G}}(g)=\mathrm{r}(h)$ and $\mathrm{s}_{\mathrm{H}}(h)=\mathrm{r}_{\mathrm{H}}\left(h_{1}\right)$; that is, the following diagram commutes:

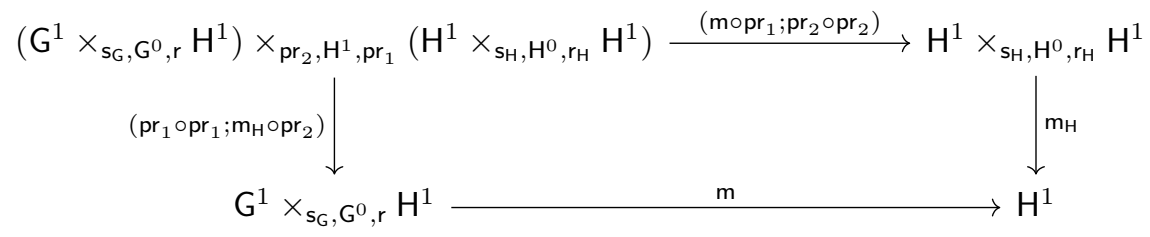

The following lemma allows us to compose actors.

Lemma 6.2. Let $\left(\mathrm{m}_{1} ; \mathrm{r}_{1}\right): \mathrm{G} \rightarrow \mathrm{H}$ and $\left(\mathrm{m}_{2} ; \mathrm{r}_{2}\right): \mathrm{H} \rightarrow \mathrm{L}$ be actors between groupoids. The pair $(\mathrm{m} ; \mathrm{r})$, where $\mathrm{r}=\mathrm{r}_{1} \circ \mathrm{u}_{\mathrm{H}} \circ \mathrm{r}_{2}$, elementwise $\mathrm{r}(l)=\mathrm{r}_{1}\left(1_{\mathrm{r}_{2}(l)}\right)$ for all $l \in \mathrm{L}^{1}$ and $\mathrm{m}=\mathrm{m}_{2} \circ\left(\mathrm{m}_{1} \circ\left(\mathrm{pr}_{1} ; \mathrm{u}_{\mathrm{H}} \circ \mathrm{r}_{2} \circ \mathrm{pr}_{2}\right) ; \mathrm{pr}_{2}\right)$, elementwise $g \cdot l=\left(g \cdot 1_{\mathrm{r}_{2}(l)}\right) \cdot l$, for all $g \in \mathrm{G}^{1}, l \in \mathrm{L}^{1}$ with $\mathrm{s}_{\mathrm{G}}(g)=\mathrm{r}(l)$ defines an actor from $\mathrm{G}$ to $\mathrm{L}$. 
Proof. We have to check the properties required in Definition 6.1

$$
\begin{aligned}
\mathrm{s}_{\mathrm{L}}(g \cdot l) & =\mathrm{s}_{\mathrm{L}}\left(\left(g \cdot 1_{\mathrm{r}_{2}(l)}\right) \cdot l\right) \\
& =\mathrm{s}_{\mathrm{L}}(l)
\end{aligned}
$$

for all $g \in \mathrm{G}^{1}, l \in \mathrm{L}^{1}$ with $\mathrm{s}_{\mathrm{G}}(g)=\mathrm{r}(l)$. That is (1) Also we have

$$
\begin{aligned}
\mathrm{r}\left(l \cdot l_{1}\right) & =\mathrm{r}_{1}\left(1_{\mathrm{r}_{2}\left(l \cdot l_{1}\right)}\right) \\
& =\mathrm{r}_{1}\left(1_{\mathrm{r}_{2}(l)}\right) \\
& =\mathrm{r}(l)
\end{aligned}
$$

for all $l, l_{1} \in \mathrm{L}^{1}$ with $\mathrm{s}_{\mathrm{L}}(l)=\mathrm{r}_{\mathrm{L}}\left(l_{1}\right)$. Hence the property $(2)$ is satisfied. In case of the property $(3)$ we compute

$$
\begin{aligned}
(g \cdot l) \cdot l_{1} & =\left(\left(g \cdot 1_{\mathrm{r}_{2}(l)}\right) \cdot l\right) \cdot l_{1} \\
& =\left(g \cdot 1_{\mathrm{r}_{2}(l)}\right) \cdot\left(l \cdot l_{1}\right) \\
& =\left(g \cdot 1_{\mathrm{r}_{2}\left(l \cdot l_{1}\right)}\right) \cdot\left(l \cdot l_{1}\right) \\
& =g \cdot\left(l \cdot l_{1}\right)
\end{aligned}
$$

for all $g \in \mathrm{G}^{1}, l, l_{1} \in \mathrm{L}^{1}$ with $\mathrm{s}_{\mathrm{G}}(g)=\mathrm{r}(l)$ and $\mathrm{s}_{\mathrm{L}}(l)=\mathrm{r}_{\mathrm{L}}\left(l_{1}\right)$. Therefore, $(\mathrm{m} ; \mathrm{r})$ is an actor from $G$ to $L$.

Example 6.3. If $\mathrm{G}$ is a groupoid then the left and right translation actions of $\mathrm{G}$ on $\mathrm{G}^{1}$ commute. Therefore, $\left(\mathrm{m}_{\mathrm{G}} ; \mathrm{r}_{\mathrm{G}}\right)$ is an actor from $\mathrm{G}$ to itself.

It is easy to check that the actor described in Example 6.3 is an identity actor in the sense of the composition defined in Lemma 6.2. Therefore, groupoids and actors between them form a category.

Generally, actors do not come from functors and vice versa. The following example describes actors and functors that are associated to each other.

Example 6.4. Let $\mathrm{F}: \mathrm{G} \rightarrow \mathrm{H}$ be a functor with invertible $\mathrm{F}^{0}$. Then the pair $(\mathrm{m} ; \mathrm{r})$, where $\mathrm{m}=\mathrm{m}_{\mathrm{H}} \circ\left(\mathrm{F}^{1} \circ \mathrm{pr}_{1} ; \mathrm{pr}_{2}\right)$ and $\mathrm{r}=\left(\mathrm{F}^{0}\right)^{-1} \circ \mathrm{r}_{\mathrm{H}}$, defines an actor from $\mathrm{G}$ to $\mathrm{H}$. Conversely, if we have an actor $(\mathrm{m} ; \mathrm{r})$ from $\mathrm{G}$ to $\mathrm{H}$ and if $\mathrm{r} \circ \mathrm{u}_{\mathrm{H}}$ is invertible then this actor comes from the functor $\mathrm{F}: \mathrm{G} \rightarrow \mathrm{H}$, defined by $\mathrm{F}^{0}=\left(\mathrm{r} \circ \mathrm{u}_{\mathrm{H}}\right)^{-1}$ and $\mathrm{F}^{1}=\mathrm{m} \circ\left(\mathrm{id}_{\mathrm{G}^{1}} ; \mathrm{u}_{\mathrm{H}} \circ\left(\mathrm{r} \circ \mathrm{u}_{\mathrm{H}}\right)^{-1} \circ \mathrm{s}_{\mathrm{G}}\right)$.

Example 6.5. Given partial groupoid fibration $\mathrm{F}: \mathrm{L} \rightarrow \mathrm{H}$ there is a functor $\left(\mathrm{pr}_{1} ; \mathrm{id}_{\mathrm{L}^{0}}\right)$ from the fibre to $\mathrm{L}$. Therefore, $\left(\mathrm{m}_{\mathrm{L}} \circ\left(\mathrm{pr}_{1} \circ \mathrm{pr}_{1} ; \mathrm{pr}_{2}\right) ; \mathrm{r}_{\mathrm{L}}\right)$ defines an actor from the fibre of $F$ to $L$.

6.2. Bibundle actors. The generalised morphisms of groupoids described in this subsection are a special kind of bibundles of groupoids. Let $G=\left(G^{0}, G^{1}, r_{G}, s_{G}, m_{G}\right)$ and $\mathrm{H}=\left(\mathrm{H}^{0}, \mathrm{H}^{1}, \mathrm{r}_{\mathrm{H}}, \mathrm{s}_{\mathrm{H}}, \mathrm{m}_{\mathrm{H}}\right)$ be groupoids.

Definition 6.6. 47, Definition 2.1] A G, H-bibundle is $\left(X ; m_{1 X} ; m_{2 X} ; s_{X} ; r_{X}\right)$, where $\left(X ; m_{1 X} ; r_{X}\right)$ is a left $G$-bundle over $s_{X}: X \rightarrow H^{0}$ and $\left(X ; m_{2 X} ; s_{X}\right)$ is a right $H$-bundle over $r_{X}: X \rightarrow G^{0}$ such that the action maps commute: $m_{2 X} \circ\left(m_{1 X} \circ \mathrm{pr}_{1} ; \mathrm{pr}_{2} \circ \mathrm{pr}_{2}\right)=$ $\mathrm{m}_{1 \mathrm{X}} \circ\left(\mathrm{pr}_{1} \circ \mathrm{pr}_{1} ; \mathrm{m}_{2 \mathrm{X}} \circ \mathrm{pr}_{2}\right)$. Elementwise $(g \cdot x) \cdot h=g \cdot(x \cdot h)$ for all $g \in \mathrm{G}^{1}, x \in \mathrm{X}$, $h \in \mathrm{H}^{1}$ with $\mathrm{s}_{\mathrm{G}}(g)=\mathrm{r}_{\mathbf{X}}(x)$ and $\mathrm{s}_{\mathrm{X}}(x)=\mathrm{r}_{\mathrm{H}}(h)$. That is, the following diagram commutes

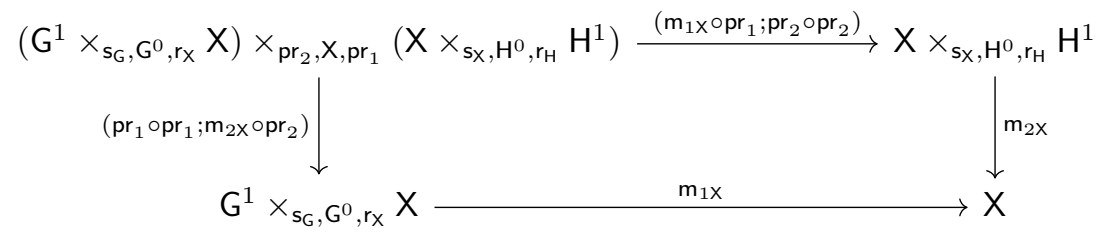


An arrow $f: \tilde{X} \rightarrow \mathbf{X}$ is called a $\mathbf{G}, \mathrm{H}$-bibundle map between two $\mathbf{G}, \mathrm{H}$-bibundles $\left(\tilde{X} ; m_{1 \tilde{X}} ; m_{2 \tilde{X}} ; s_{\tilde{X}} ; r_{\tilde{X}}\right)$ and $\left(X ; m_{1 X} ; m_{2 X} ; s \mathbf{X} ; r_{X}\right)$ if it is a $\mathrm{G}$-bundle and an H-bundle map.

Definition 6.7. A (partial) bibundle actor from $\mathrm{G}$ to $\mathrm{H}$ is the $\mathrm{G}, \mathrm{H}$-bibundle $\left(\mathrm{X} ; \mathrm{m}_{1 \mathrm{X}} ; \mathrm{m}_{2 \mathrm{X}} ; \mathrm{s}_{\mathrm{X}} ; \mathrm{r}_{\mathrm{X}}\right)$, where the right $\mathrm{H}$-action $\left(\mathrm{X} ; \mathrm{m}_{2 \mathrm{X}} ; \mathrm{s}_{\mathrm{X}}\right)$ is basic and a (partial) sheaf.

An important example is the bibundle actor from the fibre of a groupoid fibration to itself.

Example 6.8. Let $\mathrm{F}: \mathrm{L} \rightarrow \mathrm{H}$ be a groupoid fibration with fibre $\mathrm{G}$. The left and right G-actions $\left(\mathrm{L}^{1} ; \mathrm{m} ; \mathrm{r}\right)$ and $\left(\mathrm{L}^{1} ; \mathrm{m}^{\prime} ; \mathrm{s}\right)$, described in Example 5.27 define a $\mathrm{G}, \mathrm{G}$-bibundle $\left(\mathrm{L}^{1} ; \mathrm{m} ; \mathrm{m}^{\prime} ; \mathrm{s} ; \mathrm{r}\right)$ because $\mathrm{s}\left(g \cdot l_{1}\right)=\mathrm{s}\left(l_{1}\right), \mathrm{r}(l \cdot g)=\mathrm{r}(l)$ and $l \cdot\left(g \cdot l_{1}\right)=(l \cdot g) \cdot l_{1}$ for all $g \in \mathrm{G}^{1}, l, l_{1} \in \mathrm{L}^{1}$ with $\mathrm{s}_{\mathrm{L}}(g)=\mathrm{r}_{\mathrm{L}}\left(l_{1}\right)$ and $\mathrm{s}_{\mathrm{L}}(l)=\mathrm{r}_{\mathrm{L}}(g)$. It is a bibundle actor because $\mathrm{s}=\mathrm{s}_{\mathrm{L}}$ is a cover and $\left(\mathrm{L}^{1} ; \mathrm{m}^{\prime} ; \mathrm{s}\right)$ is basic by Example 5.43

Lemma 6.9. Under Assumptions 2.15 and 5.63, a partial bibundle actor from $\mathrm{G}$ to $\mathrm{H}$ induces a functor from the category of right $\mathrm{H}$-actions to the category of left G-actions:

$$
\mathcal{C}(\mathrm{H}) \rightarrow \mathcal{C}(\mathrm{G}), \quad \mathrm{Y} \mapsto \mathrm{X} \times{ }_{\mathrm{H}} \mathrm{Y} .
$$

Proof. Let $\left(\mathrm{X} ; \mathrm{m}_{1 \mathrm{X}} ; \mathrm{m}_{2 \mathrm{X}} ; \mathrm{s}_{\mathrm{X}} ; \mathrm{r}_{\mathrm{X}}\right)$ be a bibundle actor from $\mathrm{G}$ to $\mathrm{H}$ and let $\left(\mathrm{Y} ; \mathrm{m}_{\mathrm{Y}} ; \mathrm{s}_{\mathrm{Y}}\right)$

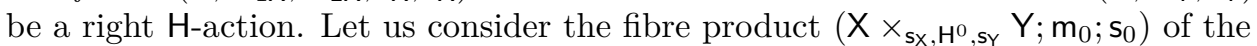
right $\mathrm{H}$-actions $\left(\mathrm{X} ; \mathrm{m}_{2 \mathrm{X}} ; \mathrm{s}_{\mathrm{X}}\right)$ and $\left(\mathrm{Y} ; \mathrm{m}_{\mathrm{Y}} ; \mathrm{s}_{\mathrm{Y}}\right.$ ) (see Example 5.25). It exists because the anchor map $s_{X}: X--\rightarrow H^{0}$ is a partial cover. Also, we can consider the pull-back

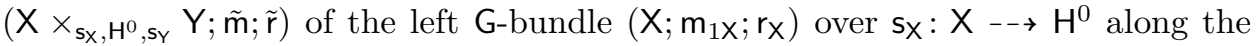

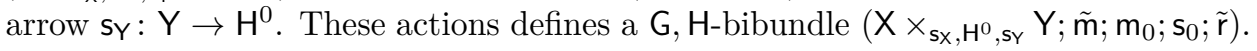
We need to check that the action maps $\tilde{\mathrm{m}}$ and $\mathrm{m}_{0}$ commute. We have

$$
\begin{aligned}
g \cdot((x ; y) \cdot h) & =g \cdot(x \cdot h ; y \cdot h) \\
& =(g \cdot(x \cdot h) ; y \cdot h) \\
& =((g \cdot x) \cdot h ; y \cdot h) \\
& =(g \cdot x ; y) \cdot h \\
& =(g \cdot(x ; y)) \cdot h
\end{aligned}
$$

for all $x \in \mathrm{X}, y \in \mathrm{Y}, g \in \mathrm{G}, h \in \mathrm{H}$ with $\mathrm{s}_{\mathbf{X}}(x)=\mathrm{s}_{\mathbf{Y}}(y)=\mathrm{r}_{\mathrm{H}}(h)$ and $\mathrm{r}_{\mathbf{X}}(x)=\mathrm{s}_{\mathrm{G}}(g)$.

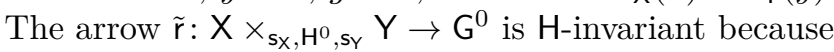

$$
\begin{aligned}
\tilde{r}((x ; y) \cdot h) & =r_{\mathbf{X}}\left(\operatorname{pr}_{1}((x ; y) \cdot h)\right) \\
& =r_{X}\left(\operatorname{pr}_{1}(x \cdot h ; y \cdot h)\right) \\
& =r_{X}(x \cdot h) \\
& =r_{X}(x) \\
& =r_{X}\left(\operatorname{pr}_{1}(x ; y)\right) \\
& =\tilde{r}(x ; y)
\end{aligned}
$$

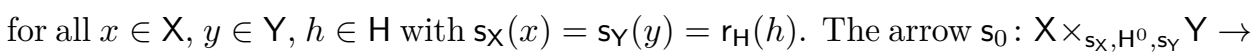
$\mathrm{H}$ is $\mathrm{G}$-invariant because

$$
\begin{aligned}
\mathrm{s}_{0}(g \cdot(x ; y)) & =\mathrm{s}_{\mathbf{X}}\left(\operatorname{pr}_{1}(g \cdot(x ; y))\right. \\
& =\mathrm{s}_{\mathbf{X}}\left(\operatorname{pr}_{1}(g \cdot x ; y)\right) \\
& =\mathrm{s}_{\mathbf{X}}(g \cdot x) \\
& =\mathrm{s}_{\mathbf{X}}(x) \\
& =\mathrm{s}_{\mathbf{X}}\left(\operatorname{pr}_{1}(x ; y)\right) \\
& =\mathrm{s}_{0}(x ; y)
\end{aligned}
$$


for all $x \in \mathrm{X}, y \in \mathrm{Y}, g \in \mathrm{G}$ with $\mathrm{s}_{\mathbf{X}}(x)=\mathrm{s}_{\mathbf{Y}}(y)$ and $\mathrm{r}_{\mathbf{X}}(x)=\mathrm{s}_{\mathrm{G}}(g)$.

We know that the $\mathrm{H}$-action $\left(X ; m_{2 X} ; s_{X}\right)$ is basic and the coordinate projection

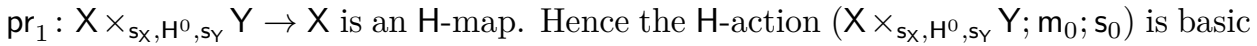
by Lemma 5.64. Therefore, there are an orbit space, denote it by $X_{X_{H}} Y$, and an

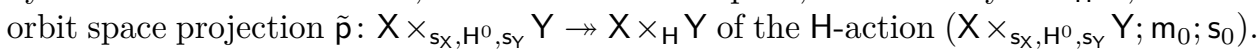
We are going to define the left action of $G$ on $X \times_{H} Y$.

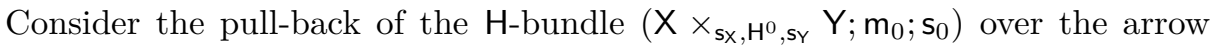
$\tilde{r}=r_{X} \circ p_{1}: X \times_{s_{X}, H^{0}, s_{Y}} Y \rightarrow G^{0}$ along the cover $s_{G}: G^{1} \rightarrow G_{0}$. It is a principal

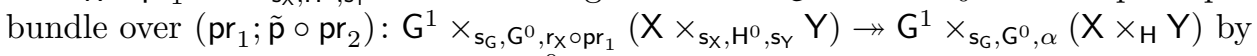
Lemma 5.50, where $\alpha: \mathrm{X} \times{ }_{\mathrm{H}} \mathrm{Y} \rightarrow \mathrm{G}^{0}$ is the unique arrow such that $\tilde{\mathrm{r}}=\alpha \circ \tilde{\mathrm{p}}$. Such $\alpha$ exists because $\tilde{\mathrm{r}}$ is $\mathrm{H}$-invariant, and it is defined elementwise by $\alpha([x ; y])=\mathrm{r}_{\mathbf{X}}(x)$.

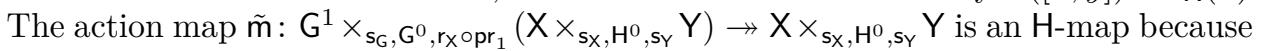

$$
(g \cdot(x ; y)) \cdot h=g \cdot((x ; y) \cdot h) .
$$

Therefore, it induces an arrow $\mathrm{m}: \mathrm{G}^{1} \times_{\mathrm{sG}_{\mathrm{G}} \mathrm{G}^{0}, \alpha}\left(\mathrm{X} \times \times_{\mathrm{H}} \mathrm{Y}\right) \rightarrow \mathrm{X} \times_{\mathrm{H}} \mathrm{Y}$ such that $\mathrm{m} \circ$ $\left(\mathrm{pr}_{1} ; \tilde{\mathrm{p}} \circ \mathrm{pr}_{2}\right)=\tilde{\mathrm{p}} \circ \tilde{\mathrm{m}}$. Elementwise $g \cdot[x ; y]=[g \cdot x ; y]$. Now, we need to show that the triple $\left(\mathrm{X} \times_{\mathrm{H}} \mathrm{Y} ; \mathrm{m} ; \alpha\right)$ defines a left $\mathrm{G}$-action. We have

$$
\begin{aligned}
\alpha(g \cdot[x ; y]) & =\alpha([g \cdot x ; y]) \\
& =\mathrm{r}_{\mathbf{X}}(g \cdot x) \\
& =\mathrm{r}_{\mathrm{G}}(g)
\end{aligned}
$$

for all $x \in \mathrm{X}, y \in \mathrm{Y}, g \in \mathrm{G}^{1}$ with $\mathrm{s}_{\mathbf{X}}(x)=\mathrm{s}_{\mathrm{L}}(y)$ and $=\mathrm{r}_{\mathrm{X}}(x)=\mathrm{s}_{\mathrm{G}}(g)$. Since the arrow $\left(\mathrm{pr}_{1} ; \tilde{\mathrm{p}} \circ \mathrm{pr}_{2}\right)$ is a cover, it is a coequaliser and therefore, it is epic. Thus $\alpha \circ \mathrm{m}=\mathrm{r}_{\mathrm{G}} \circ \mathrm{pr}_{1}$. This is property (1)

There is a diagram of pull-back squares

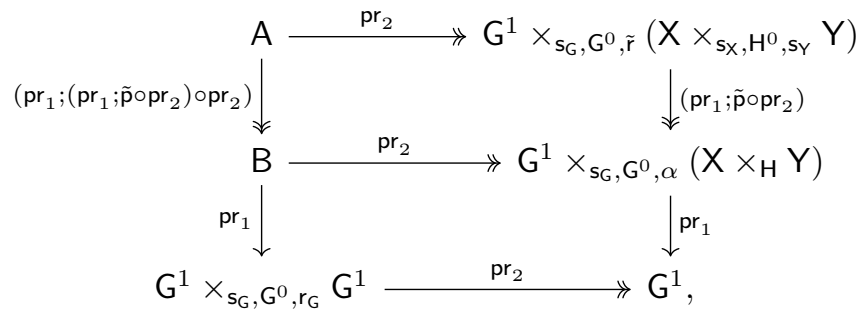

where the object $A$ is $\left(G^{1} \times_{s_{G}, G^{0}, r_{G}} G^{1}\right) \times_{p r_{2}, G^{1}, p r_{1}}\left(G^{1} \times_{s_{G}, G^{0}, \tilde{r}}\left(X \times_{s_{X}, H^{0}, s_{Y}} Y\right)\right)$ and $B$ is $\left(G^{1} \times_{s_{G}, G^{0}, r_{G}} G^{1}\right) \times_{p r_{2}, G^{1}, p r_{1}}\left(G^{1} \times_{s_{G}, G^{0}, \tilde{r}}\left(X \times_{H} Y\right)\right)$. Since the upper square is a pull-back square, the arrow $\left(\mathrm{pr}_{1} ;\left(\mathrm{pr}_{1} ; \tilde{\mathrm{p}} \circ \mathrm{pr}_{2}\right) \circ \mathrm{pr}_{2}\right): \mathrm{A} \rightarrow \mathrm{B}$ is a cover by Assumption 2.15 because it is a pull-back of the cover $\left(\mathrm{pr}_{1} ; \tilde{\mathrm{p}} \circ \mathrm{pr}_{2}\right)$. Therefore, $\left(\mathrm{pr}_{1} ;\left(\mathrm{pr}_{1} ; \tilde{\mathrm{p}} \circ \mathrm{pr}_{2}\right) \circ \mathrm{pr}_{2}\right)$ is epic. We also have

$$
\begin{aligned}
g_{1} \cdot(g \cdot[x ; y]) & =g_{1} \cdot[g \cdot x ; y] \\
& =\left[g_{1} \cdot(g \cdot x) ; y\right] \\
& =\left[\left(g_{1} \cdot g\right) \cdot x ; y\right] \\
& =\left(g_{1} \cdot g\right) \cdot[x ; y]
\end{aligned}
$$

for all $x \in \mathrm{X}, y \in \mathrm{Y}, g, g_{1} \in \mathrm{G}^{1}$ with $\mathrm{s}_{\mathrm{X}}(x)=\mathrm{s}_{\mathrm{Y}}(y), \mathrm{r}_{\mathrm{X}}(x)=\mathrm{s}_{\mathrm{G}}(g)$ and $\mathrm{s}_{\mathrm{G}}\left(g_{1}\right)=\mathrm{r}_{\mathrm{G}}(g)$. Since the arrow $\left(\mathrm{pr}_{1} ;\left(\mathrm{pr}_{1} ; \tilde{\mathrm{p}} \circ \mathrm{pr}_{2}\right) \circ \mathrm{pr}_{2}\right)$ is epic, the property (2) holds. That is, $\mathrm{m} \circ\left(\mathrm{pr}_{1} \circ \mathrm{pr}_{1} ; \mathrm{m} \circ \mathrm{pr}_{2}\right)=\mathrm{m} \circ\left(\mathrm{m}_{\mathrm{G}} \circ \mathrm{pr}_{1} ; \mathrm{pr}_{2} \circ \mathrm{pr}_{2}\right)$. We need one more property:

$$
\begin{aligned}
1_{\alpha([x ; y])} \cdot[x ; y] & =1_{\mathrm{r}_{\mathrm{X}}(x)} \cdot[x ; y] \\
& =\left[1_{\mathrm{r}_{\mathbf{X}}(x)} \cdot x ; y\right] \\
& =[x ; y]
\end{aligned}
$$




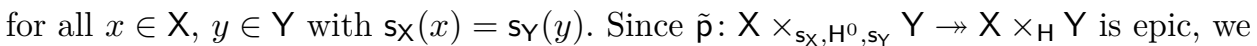
have $\mathrm{m} \circ\left(\mathrm{u}_{\mathrm{G}} \circ \alpha ; \mathrm{id}_{\mathrm{X}}\right)=\mathrm{id} \mathrm{X}$. Therefore, the triple $\left(\mathrm{X} \times_{\mathrm{H}} \mathrm{Y} ; \mathrm{m} ; \alpha\right)$ is a left G-action by Remark 5.11 .

Now, consider any $\mathrm{H}$-map $f: \mathrm{Y}_{1} \rightarrow \mathrm{Y}_{2}$. Since $\mathrm{s}_{\mathrm{Y}_{2}} \circ f=\mathrm{s}_{\mathrm{Y}_{1}}$, we have a well-defined

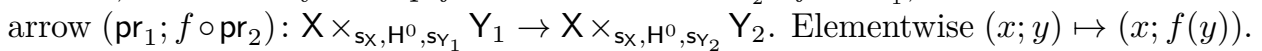
It is a G-map because it clearly commutes with the anchor maps and also we have $g \cdot(x ; f(y))=(g \cdot x ; f(y))$. It is an $\mathrm{H}$-map, too, because

$$
\begin{aligned}
(x ; f(y)) \cdot h & =(x \cdot h ; f(y) \cdot h) \\
& =(x \cdot h ; f(y \cdot h))
\end{aligned}
$$

for all $x \in \mathrm{X}, y \in \mathrm{Y}_{1}, h \in \mathrm{H}^{1}$ with $\mathrm{sX}_{\mathbf{X}}(x)=\mathrm{sY}_{1}(y)=\mathrm{r}_{\mathrm{H}}(h)$ Therefore, the $\mathrm{H}$-map $\left(\mathrm{pr}_{1} ; f \circ \mathrm{pr}_{2}\right)$ induces an arrow $\tilde{f}: \mathrm{X} \times_{\mathrm{H}} \mathrm{Y}_{1} \rightarrow \mathrm{X} \times_{\mathrm{H}} \mathrm{Y}_{2}$ such that

$$
\tilde{f} \circ \tilde{\mathrm{p}}_{1}=\tilde{\mathrm{p}}_{2} \circ\left(\mathrm{pr}_{1} ; f \circ \mathrm{pr}_{2}\right), \quad \tilde{f}([x ; y])=[x ; f(y)] .
$$

Since the cover $\left(\mathrm{pr}_{1} ; \tilde{\mathrm{p}}_{1} \circ \mathrm{pr}_{2}\right): \mathrm{G}^{1} \times_{\mathrm{sG}_{\mathrm{G}}, \mathrm{G}^{0}, \tilde{r}_{1}}\left(\mathrm{X} \times_{\mathrm{sX}_{\mathrm{X}}, \mathrm{H}^{0}, \mathrm{sY}_{1}} \mathrm{Y}_{1}\right) \rightarrow \mathrm{G}^{1} \times_{\mathrm{sG}_{\mathrm{G}}, \mathrm{G}^{0}, \alpha_{1}}\left(\mathrm{X} \times \times_{\mathrm{H}} \mathrm{Y}_{1}\right)$ is epic and

$$
\begin{aligned}
\tilde{f}(g \cdot[x ; y]) & =\tilde{f}([g \cdot x ; y]) \\
& =[g \cdot x ; f(y)] \\
& =g \cdot[x ; f(y)] \\
& =\tilde{f}([x ; y])
\end{aligned}
$$

for all $x \in \mathrm{X}, y \in \mathrm{Y}_{1}, g \in \mathrm{G}^{1}$ with $\mathrm{s}_{\mathrm{X}}(x)=\mathrm{s}_{\mathrm{Y}_{1}}(y)$ and $\mathrm{r}_{\mathrm{X}}(x)=\mathrm{s}_{\mathrm{H}}(g)$, we have that $\tilde{f} \circ \mathrm{m}_{1}=\mathrm{m}_{2} \circ\left(\mathrm{pr}_{1} ; \tilde{f} \circ \mathrm{pr}_{2}\right)$. Also

$$
\begin{aligned}
\alpha_{2}(\tilde{f}([x ; y])) & =\alpha_{2}([x ; f(y)]) \\
& =r_{X}(x) \\
& =\alpha_{1}([x ; y])
\end{aligned}
$$

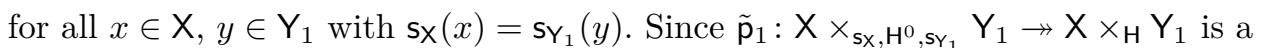
cover, it is epic and therefore, we have $\alpha_{2} \circ \tilde{f}=\alpha_{1}$. Thus an $\mathrm{H}$-map $f: \mathrm{Y}_{1} \rightarrow \mathrm{Y}_{2}$ gives a G-map $\tilde{f}: \mathrm{X} \times_{\mathrm{H}} \mathrm{Y}_{1} \rightarrow \mathrm{X} \times \times_{\mathrm{H}} \mathrm{Y}_{2}$.

The last step is to show that

$$
\widetilde{f_{2} \circ f_{1}}=\tilde{f}_{2} \circ \tilde{f}_{1} \text {. }
$$

Consider two composable $\mathrm{H}$-maps $f_{1}: \mathrm{Y}_{1} \rightarrow \mathrm{Y}_{2}$ and $f_{2}: \mathrm{Y}_{2} \rightarrow \mathrm{Y}_{3}$.

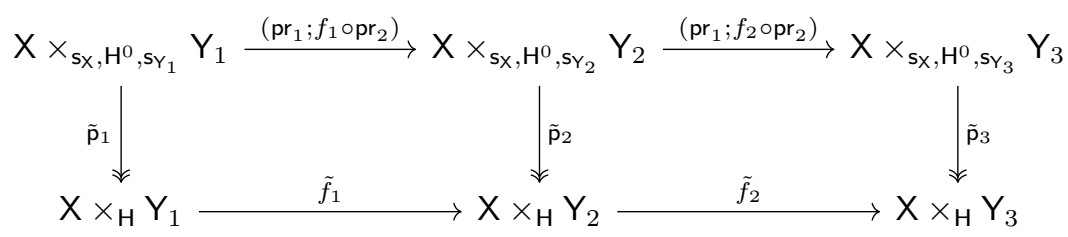

We have

$$
\begin{aligned}
\widetilde{f_{2} \circ f_{1}}([x ; y]) & =\left[x ; f_{2}\left(f_{1}(y)\right)\right] \\
& =\tilde{f}_{2}\left(\left[x ; f_{1}(y)\right]\right) \\
& =\tilde{f}_{2}\left(\tilde{f}_{1}([x ; y])\right) \\
& =\left(\tilde{f}_{2} \circ \tilde{f}_{1}\right)([x ; y])
\end{aligned}
$$

for all $x \in \mathrm{X}, y \in \mathrm{Y}_{1}$ with $\mathrm{sX}_{\mathbf{X}}(x)=\mathrm{s}_{1}(y)$. The arrow $\left(\mathrm{pr}_{1} ; f_{2} \circ f_{1} \circ \mathrm{pr}_{2}\right)$ induces a unique arrow $\widehat{f_{2} \circ f_{1}}$ such that the suitable square commutes by Lemma 5.51 . Therefore, $\widetilde{f_{2} \circ f_{1}}=\tilde{f}_{2} \circ \tilde{f}_{1}$. 
Remark 6.10. Let $\left(\mathrm{X} ; \mathrm{m}_{1 \mathrm{X}} ; \mathrm{m}_{2 \mathrm{X}} ; \mathrm{sX} ; \mathrm{rX}\right)$ and $\left(\mathrm{X}_{1} ; \mathrm{m}_{1 \mathrm{X}_{1}} ; \mathrm{m}_{2 \mathrm{X}_{1}} ; \mathrm{sX}_{1} ; \mathrm{r}_{\mathrm{X}_{1}}\right)$ be partial bibundle actors from $\mathrm{G}$ to $\mathrm{H}$. A G, $\mathrm{H}$-map $f: \mathrm{X} \rightarrow \mathrm{X}_{1}$ induces a $\mathrm{G}$-map $\hat{f}: \mathrm{X}_{\times_{\mathrm{H}} \mathrm{Y}} \rightarrow \mathrm{X}_{1} \times{ }_{\mathrm{H}} \mathrm{Y}$. The arrow

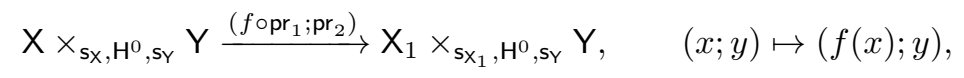

is an $\mathrm{H}$-map because

$$
\begin{aligned}
\left(f \circ \mathrm{pr}_{1} ; \mathrm{pr}_{2}\right)((x ; y) \cdot h) & =\left(f \circ \mathrm{pr}_{1} ; \mathrm{pr}_{2}\right)(x \cdot h ; y \cdot h) \\
& =(f(x \cdot h) ; y \cdot h) \\
& =(f(x) \cdot h ; y \cdot h) \\
& =(f(x) ; y) \cdot h \\
& =\left(f \circ \mathrm{pr}_{1} ; \mathrm{pr}_{2}\right)(x ; y) \cdot h
\end{aligned}
$$

for all $x \in \mathrm{X}, y \in \mathrm{Y}, h \in \mathrm{H}^{1}$ with $\mathrm{s}_{\mathbf{X}}(x)=\mathrm{s}_{\mathrm{Y}}(y)=\mathrm{r}_{\mathrm{H}}(h)$. Therefore, it induces a unique arrow $\hat{f}: \mathrm{X} \times_{\mathrm{H}} \mathrm{Y} \rightarrow \mathrm{X}_{1} \times_{\mathrm{H}} \mathrm{Y}$ such that $\hat{f} \circ \tilde{\mathrm{p}}=\tilde{\mathrm{p}}_{1} \circ\left(f \circ \mathrm{pr}_{1} ; \mathrm{pr}_{2}\right)$. Elementwise $\hat{f}([x ; y])=[f(x) ; y]$. This arrow is a G-map because

$$
\begin{aligned}
\hat{f}(g \cdot[x ; y]) & =\hat{f}([g \cdot x ; y]) \\
& =[f(g \cdot x) ; y] \\
& =[g \cdot f(x) ; y] \\
& =g \cdot[f(x) ; y] \\
& =g \cdot \hat{f}([x ; y])
\end{aligned}
$$

for all $x \in \mathrm{X}, y \in \mathrm{Y}, g \in \mathrm{G}^{1}$ with $\mathrm{s}_{\mathbf{X}}(x)=\mathrm{s}_{\mathrm{Y}}(y)=\mathrm{r}_{\mathrm{H}}(h)$ and $\mathrm{r}_{\mathbf{X}}(x)=\mathrm{s}_{\mathrm{G}}(g)$.

6.3. Bibundle functor. Let $\mathrm{G}=\left(\mathrm{G}^{0} ; \mathrm{G}^{1} ; \mathrm{r}_{\mathrm{G}} ; \mathrm{s}_{\mathrm{G}} ; \mathrm{m}_{\mathrm{G}}\right)$ and $\mathrm{H}=\left(\mathrm{H}^{0} ; \mathrm{H}^{1} ; \mathrm{r}_{\mathrm{H}} ; \mathrm{s}_{\mathrm{H}} ; \mathrm{m}_{\mathrm{H}}\right)$ be groupoids in a category $\left(\mathcal{C}, \mathcal{F}_{p}\right)$ with partial covers.

Definition 6.11. A (partial) bibundle functor from $\mathrm{G}$ to $\mathrm{H}$ is a $\mathrm{G}, \mathrm{H}$-bibundle $\left(X ; \mathrm{m}_{1 X} ; \mathrm{m}_{2 \mathrm{X}} ; \mathrm{sX} ; \mathrm{rX}\right)$, where the right $\mathrm{H}$-bundle $\left(\mathrm{X} ; \mathrm{m}_{2 \mathrm{X}} ; \mathrm{sX}_{\mathrm{X}}\right)$ is (partially) principal over $r_{X}: X \rightarrow G^{0}$. A bibundle functor from $G$ to $H$ is a (partially) covering if the arrow $\mathrm{s}_{\mathrm{X}}: \mathrm{X} \rightarrow \mathrm{H}^{0}$ is a (partial) cover.

An important example is the bibundle functor from $\mathrm{G}$ to $\mathrm{H}$ associated to the functor $\mathrm{F}: \mathrm{G} \rightarrow \mathrm{H}$.

Example 6.12. Let $\mathrm{F}: \mathrm{G} \rightarrow \mathrm{H}$ be a functor between groupoids. There is a bibundle functor $\left(X ; m_{1 X} ; m_{2 X} ; s_{X} ; r_{X}\right)$ from $G$ to $H$, where the left $G$-action $\left(X ; m_{1 X} ; r_{X}\right)$ is the $\mathrm{G}$-sheaf described in Example 5.26 and the right $\mathrm{H}$-bundle $\left(\mathrm{X} ; \mathrm{m}_{2 \mathrm{X}} ; \mathrm{sX}\right)$ is the pull-back of the principal $\mathrm{H}$-bundle described in Example 5.42 along the arrow $\mathrm{F}^{0}: \mathrm{G}^{0} \rightarrow \mathrm{H}^{0}$. We have to check that the multiplication maps commute. We have

$$
\begin{aligned}
\left(g \cdot\left(g_{0} ; h\right)\right) \cdot h_{1} & =\left(\mathrm{r}_{\mathrm{G}}(g) ; h\right) \cdot h_{1} \\
& =\left(\mathrm{r}_{\mathrm{G}}(g) ; h \cdot h_{1}\right) \\
& =g \cdot\left(g_{0} ; h \cdot h_{1}\right) \\
& =g \cdot\left(\left(g_{0} ; h\right) \cdot h_{1}\right)
\end{aligned}
$$

for all $g \in \mathrm{G}^{1}, g_{0} \in \mathrm{G}^{0}, h, h_{1} \in \mathrm{H}^{1}$ with $\mathrm{s}_{\mathrm{G}}(g)=g_{0}, \mathrm{~F}^{0}\left(g_{0}\right)=\mathrm{r}_{\mathrm{H}}(h)$ and $\mathrm{s}_{\mathrm{H}}(h)=$ $\mathrm{r}_{\mathrm{H}}\left(h_{1}\right)$. The anchor map $\mathrm{s}_{\mathrm{X}}=\mathrm{s}_{\mathrm{H}} \circ \mathrm{pr}_{2}$ is $\mathrm{G}$-invariant because

$$
\begin{aligned}
\mathrm{s}_{\mathbf{X}}\left(g \cdot\left(g_{0} ; h\right)\right) & =\mathrm{s}_{\mathbf{X}}\left(\mathrm{r}_{\mathrm{G}}(g) ; h\right) \\
& =\mathrm{s}_{\mathrm{H}}(h) \\
& =\mathrm{s}_{\mathbf{X}}\left(g_{0} ; h\right)
\end{aligned}
$$

for all $g \in \mathrm{G}^{1}, g_{0} \in \mathrm{G}^{0}, h \in \mathrm{H}^{1}$ with $\mathrm{s}_{\mathrm{G}}(g)=g_{0}$ and $\mathrm{F}^{0}\left(g_{0}\right)=\mathrm{r}_{\mathrm{H}}(h)$. Also, we know that the $\mathrm{H}$-bundle $\left(\mathrm{X} ; \mathrm{m}_{2 \mathrm{X}} ; \mathrm{sX}_{\mathrm{X}}\right)$ is partially principal by Lemma 5.50 . It is 
even a principal bundle because $\mathrm{r}_{\mathrm{H}}: \mathrm{H}^{1} \rightarrow \mathrm{H}^{0}$ is a splitting cover and therefore,

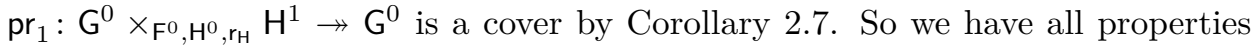
needed for a bibundle functor. So $\left(X ; \mathrm{m}_{1 \mathrm{X}} ; \mathrm{m}_{2 \mathrm{X}} ; \mathrm{sx}_{\mathrm{X}} ; \mathrm{r}_{\mathrm{X}}\right)$ defines a bibundle functor from $\mathrm{G}$ to $\mathrm{H}$. Call such a bibundle functor associated to the functor $\mathrm{F}: \mathrm{G} \rightarrow \mathrm{H}$.

Lemma 6.13. Under Assumptions 2.15 and 5.66, a bibundle functor from $\mathrm{G}$ to $\mathrm{H}$ induces a functor from the category of right $\mathrm{H}$-sheaves to the category of left G-actions:

$$
\mathcal{C}_{\mathcal{F}}(\mathrm{H}) \rightarrow \mathcal{C}(\mathrm{G}), \quad \mathrm{Y} \mapsto \mathrm{X} \times_{\mathrm{H}} \mathrm{Y} .
$$

Proof. The proof of this lemma and the proof of Lemma 6.9 are almost the same. There are two differences. The first of them is that the object $X \times_{s_{X}, H^{0}, s_{Y}} Y$ exists for different reasons: In the previous case we use that the arrow $s_{X}: X-\rightarrow H^{0}$ is a partial cover and in this case we use that the arrow $s_{Y}: \mathrm{Y} \rightarrow \mathrm{H}^{0}$ is a cover because $\left(\mathrm{Y} ; \mathrm{m}_{\mathrm{Y}} ; \mathrm{s}_{\mathrm{Y}}\right)$ is an $\mathrm{H}$-sheaf.

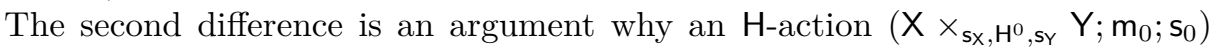
is basic. In the previous case this is Lemma 5.64. In this case the coordinate

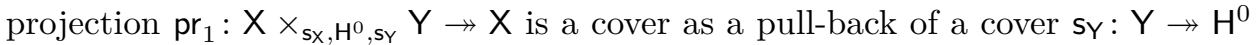

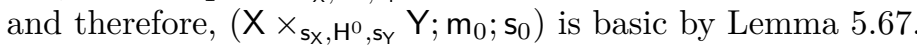

Remark 6.14. In Lemma 6.13, under Assumption 2.18 the G-action $\left(\mathrm{X} \times_{\mathrm{H}} \mathrm{Y} ; \mathrm{m} ; \alpha\right)$ is a sheaf. That is, the anchor map $\alpha: X \times_{H} Y \rightarrow G^{0}$ is a cover. This anchor map

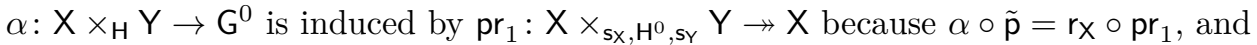

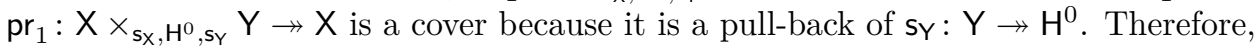
$\alpha: \mathrm{X} \times_{\mathrm{H}} \mathrm{Y} \rightarrow \mathrm{G}^{0}$ is a cover by Corollary 5.53 .

Proposition 6.15. Assume Assumptions 2.15, 2.18 and 5.66. Let $\mathrm{G}, \mathrm{H}$ and $\mathrm{K}$ be groupoids in $\left(\mathcal{C}, \mathcal{F}_{p}\right)$. Let $\left(\mathrm{X} ; \mathrm{m}_{1 \mathrm{X}} ; \mathrm{m}_{2 \mathrm{X}} ; \mathrm{s}_{\mathrm{X}} ; \mathrm{r}_{\mathrm{X}}\right) \equiv \mathbb{X}$ and $\left(\mathrm{Y} ; \mathrm{m}_{1 \mathrm{Y}} ; \mathrm{m}_{2 \mathrm{Y}} ; \mathrm{s}_{\mathrm{Y}} ; \mathrm{r}_{\mathrm{Y}}\right) \equiv \mathbb{Y}$ be bibundle functors from $\mathrm{G}$ to $\mathrm{H}$ and from $\mathrm{H}$ to $\mathrm{K}$, respectively. Then there is a $\mathrm{G}, \mathrm{K}$-bibundle functor $\left(\mathrm{Y} \circ \mathrm{X} ; \mathrm{m}_{1} ; \mathrm{m}_{2} ; \mathrm{s} ; \mathrm{r}\right)$ from $\mathrm{G}$ to $\mathrm{K}$. Call it a composition of bibundle functors $\left(\mathrm{X} ; \mathrm{m}_{1 \mathrm{X}} ; \mathrm{m}_{2 \mathrm{X}} ; \mathrm{s}_{\mathrm{X}} ; \mathrm{r}_{\mathrm{X}}\right) \equiv \mathbb{X}$ and $\left(\mathrm{Y} ; \mathrm{m}_{1 \mathrm{Y}} ; \mathrm{m}_{2 \mathrm{Y}} ; \mathrm{s}_{\mathrm{Y}} ; \mathrm{r}_{\mathrm{Y}}\right) \equiv \mathbb{Y}$ and denote it by $\mathbb{Y} \circ \mathbb{X}$.

Proof. First of all, let us define all data in $\left(\mathrm{Y} \circ \mathrm{X} ; \mathrm{m}_{1} ; \mathrm{m}_{2} ; \mathrm{s} ; \mathrm{r}\right)$. According to Lemma 5.18 we have a right $\mathrm{H}$-action $\left(\mathrm{Y} ; \mathrm{m}_{1 \mathrm{Y}} \circ\left(\mathrm{i}_{\mathrm{H}} \circ \mathrm{pr}_{2} ; \mathrm{pr}_{1}\right) ; \mathrm{r}_{\mathrm{Y}}\right)$ corresponding to the left $\mathrm{H}$-action $\left(\mathrm{Y} ; \mathrm{m}_{1 \mathrm{Y}} ; \mathrm{r}_{\mathrm{Y}}\right)$. Let $\left(\mathrm{Y} \circ \mathrm{X} ; \mathrm{m}_{1} ; \mathrm{r}\right)$ be the left $\mathrm{G}$-action which is given by the right $\mathrm{H}$-action $\left(\mathrm{Y} ; \mathrm{m}_{1 \mathrm{Y}} \circ\left(\mathrm{i}_{\mathrm{H}} \circ \mathrm{pr}_{2} ; \mathrm{pr}_{1}\right) ; \mathrm{r}_{\mathrm{Y}}\right)$ by using the functor described in Lemma 6.13. which is induced by the bibundle functor $\left(X ; m_{1 X} ; m_{2 X} ; s_{X} ; r_{X}\right)$. Also, we need to define the right $\mathrm{K}$-action $\left(\mathrm{X} \times_{\mathrm{H}} \mathrm{Y} ; \mathrm{m}_{2} ; \mathrm{s}\right)$. Consider the pull-back $\left(\mathrm{X} \times_{\mathbf{s X}_{\mathrm{X}}, \mathrm{H}^{0}, \mathrm{rY}} \mathrm{Y} ; \hat{\mathrm{m}} ; \hat{\mathrm{s}}\right)$ of the right $\mathrm{K}$-bundle $\left(\mathrm{Y} ; \mathrm{m}_{2 \mathrm{Y}} ; \mathrm{sY}_{\mathrm{Y}}\right.$ ) over $\mathrm{r}_{\mathrm{Y}}: \mathrm{Y} \rightarrow \mathrm{H}^{0}$ along the arrow $\mathrm{sX}_{\mathrm{X}}: \mathrm{X} \rightarrow \mathrm{H}^{0}$. Let us show that the anchor map $\hat{s}: X \times_{s_{X}, H^{0}, r_{Y}} Y \rightarrow K^{0}$ is $\mathrm{H}$-invariant in the sense of the right $\mathrm{H}$-action $\left(X \times_{s_{x}, H^{0}, r_{Y}} Y ; m_{0} ; s_{0}\right)$, which is used for the construction of the left $\mathrm{G}$-action $\left(X \times_{H} Y ; m_{1} ; r\right)$. This action is the fibre product of right $\mathrm{H}$-actions $\left(\mathrm{X} ; \mathrm{m}_{2 \mathrm{X}} ; \mathrm{s}_{\mathrm{X}}\right)$ and $\left(\mathrm{Y} ; \mathrm{m}_{1 \mathrm{Y}} \circ\left(\mathrm{i}_{\mathrm{H}} \circ \mathrm{pr}_{2} ; \mathrm{pr}_{1}\right) ; \mathrm{r}_{\mathrm{Y}}\right)$. We have

$$
\begin{aligned}
\hat{\mathbf{s}}((x ; y) \cdot h) & =\mathrm{sY}_{\mathbf{Y}}\left(\mathrm{pr}_{2}\left(x \cdot h ; h^{-1} \cdot y\right)\right) \\
& =\mathrm{s}_{\mathbf{Y}}\left(h^{-1} \cdot y\right) \\
& =\mathrm{sY}_{Y}(y) \\
& =\mathrm{s}_{Y}\left(\operatorname{pr}_{2}(x ; y)\right) \\
& =\hat{\mathbf{s}}(x ; y)
\end{aligned}
$$

for all $x \in \mathrm{X}, y \in \mathrm{Y}, h \in \mathrm{H}^{1}$ with $\mathrm{s}_{\mathbf{X}}(x)=\mathrm{r}_{\mathrm{Y}}(y)=\mathrm{r}_{\mathrm{H}}(h)$. Therefore, there is a unique arrow $\mathrm{s}: \mathrm{X} \times{ }_{\mathrm{H}} \mathrm{Y} \rightarrow \mathrm{K}^{0}$ such that $\mathrm{s} \circ \tilde{\mathrm{p}}=\hat{\mathrm{s}}$. Elementwise $\mathrm{s}([x ; y])=\mathrm{s}_{\mathrm{Y}}(y)$.

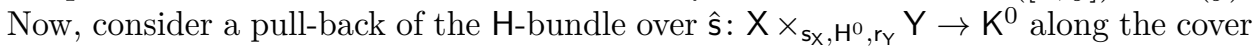


$\mathrm{r}_{\mathrm{K}}: \mathrm{K}^{1} \rightarrow \mathrm{K}^{0}$. It is a principal bundle over

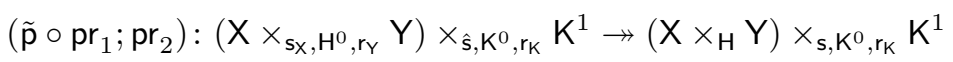

by Lemma 5.50 . Let us show that the arrow

$$
\left(X \times_{s_{X}, H^{0}, r_{Y}} Y\right) \times_{\hat{s}, K^{0}, r_{K}} K^{1} \stackrel{\hat{m}}{\rightarrow} X \times_{s_{X}, H^{0}, r_{Y}} Y
$$

is an $\mathrm{H}$-map. We have

$$
\begin{aligned}
((x ; y) \cdot h) \cdot k & =\left(x \cdot h ; h^{-1} \cdot y\right) \cdot k \\
& =\left(x \cdot h ;\left(h^{-1} \cdot y\right) \cdot k\right) \\
& =\left(x \cdot h ; h^{-1} \cdot(y \cdot k)\right) \\
& =(x ; y \cdot k) \cdot h \\
& =((x ; y) \cdot k) \cdot h
\end{aligned}
$$

forall $x \in \mathrm{X}, y \in \mathrm{Y}, h \in \mathrm{H}^{1}, k \in \mathrm{K}^{1}$ with $\mathrm{s}_{\mathbf{X}}(x)=\mathrm{r}_{\mathrm{Y}}(y)=\mathrm{r}_{\mathrm{H}}(h)$ and $\mathrm{s}_{\mathrm{Y}}(y)=\mathrm{r}_{\mathrm{K}}(k)$. Therefore, we have an induced arrow $m_{2}:\left(X \times_{H} Y\right) \times_{s, K^{0}, r_{K}} K^{1} \rightarrow X \times_{H} Y$ such that $\mathrm{m}_{2} \circ\left(\tilde{\mathrm{p}} \circ \mathrm{pr}_{1} ; \mathrm{pr}_{2}\right)=\tilde{\mathrm{p}} \circ \hat{\mathrm{m}}$. Elementwise $[x ; y] \cdot k=[x ; y \cdot k]$.

We need to show that $\left(\mathrm{X} \times_{\mathrm{H}} \mathrm{Y} ; \mathrm{m}_{2} ; \mathrm{s}\right)$ is a right $\mathrm{K}$-action. We have

$$
\begin{aligned}
\mathrm{s}([x ; y] \cdot k) & =\mathrm{s}([x ; y \cdot k]) \\
& =\mathrm{s}_{\mathrm{Y}}(y \cdot k) \\
& =\mathrm{s}_{\mathrm{K}}(k)
\end{aligned}
$$

for all $x \in \mathrm{X}, y \in \mathrm{Y}, k \in \mathrm{K}^{1}$ with $\mathrm{s}_{\mathbf{X}}(x)=\mathrm{r}_{\mathbf{Y}}(y)$ and $\mathrm{s}_{\mathrm{Y}}(y)=\mathrm{r}_{\mathrm{K}}(k)$. Since the arrow $\left(\tilde{\mathrm{p}} \circ \mathrm{pr}_{1} ; \mathrm{pr}_{2}\right)$ is a cover, it is a coequaliser and therefore, it is epic. Thus $\mathrm{s} \circ \mathrm{m}_{2}=\mathrm{s}_{\mathrm{K}} \circ \mathrm{pr}_{2}$. This is a property (1)

There is a diagram of pull-back squares

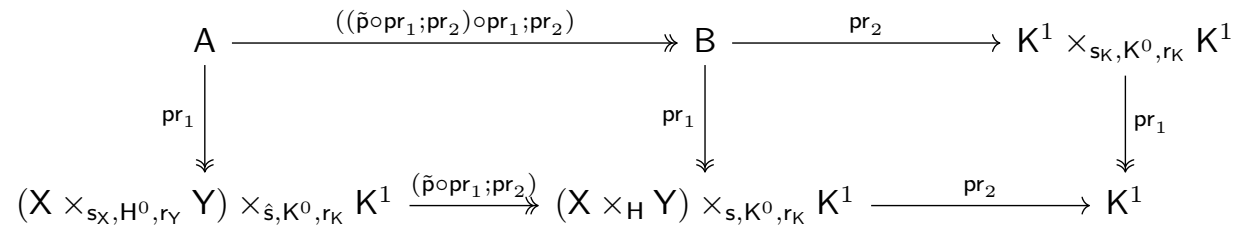

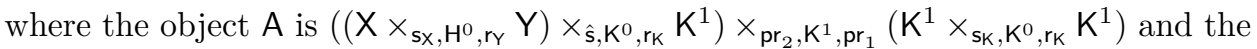
object $B$ is $\left(\left(X \times_{H} Y\right) \times_{s, K^{0}, r_{K}} K^{1}\right) \times_{p_{2}, K^{1}, p_{1}}\left(K^{1} \times_{\mathrm{sK}_{K}, K^{0}, r_{K}} K^{1}\right)$. Since the left square is a pull-back square, the arrow $\left(\left(\tilde{\mathrm{p}} \circ \mathrm{pr}_{1} ; \mathrm{pr}_{2}\right) \circ \mathrm{pr}_{1} ; \mathrm{pr}_{2}\right): \mathrm{A} \rightarrow \mathrm{B}$ is a cover by Assumption 2.15 as the pull-back of the cover $\left(\tilde{\mathrm{p}} \circ \mathrm{pr}_{1} ; \mathrm{pr}_{2}\right)$. Therefore, $\left(\left(\tilde{\mathrm{p}} \circ \mathrm{pr}_{1} ; \mathrm{pr}_{2}\right) \circ \mathrm{pr}_{1} ; \mathrm{pr}_{2}\right)$ is epic. We have

$$
\begin{aligned}
([x ; y] \cdot k) \cdot k_{1} & =[x ; y \cdot k] \cdot k_{1} \\
& =\left[x ;(y \cdot k) \cdot k_{1}\right] \\
& =\left[x ; y \cdot\left(k \cdot k_{1}\right)\right] \\
& =[x ; y] \cdot\left(k \cdot k_{1}\right)
\end{aligned}
$$

for all $x \in \mathrm{X}, y \in \mathrm{Y}, k, k_{1} \in \mathrm{K}^{1}$ with $\mathrm{s}_{\mathbf{X}}(x)=\mathrm{r}_{\mathrm{Y}}(y), \mathrm{s}_{\mathrm{Y}}(y)=\mathrm{r}_{\mathrm{K}}(k)$ and $\mathrm{s}_{\mathrm{K}}(k)=\mathrm{r}_{\mathrm{K}}\left(k_{1}\right)$. Since the arrow $\left(\left(\tilde{\mathrm{p}} \circ \mathrm{pr}_{1} ; \mathrm{pr}_{2}\right) \circ \mathrm{pr}_{1} ; \mathrm{pr}_{2}\right)$ is epic, the property (2) holds. That is: $\mathrm{m}_{2} \circ\left(\mathrm{m}_{2} \circ \mathrm{pr}_{1} ; \mathrm{pr}_{2} \circ \mathrm{pr}_{2}\right)=\mathrm{m}_{2} \circ\left(\mathrm{pr}_{1} \circ \mathrm{pr}_{1} ; \mathrm{m}_{\mathrm{K}} \circ \mathrm{pr}_{2}\right)$. We need one more property.

$$
\begin{aligned}
{[x ; y] \cdot 1_{\mathrm{s}([x ; y])} } & =\left[x ; y \cdot 1_{\mathrm{SY}(y)}\right] \\
& =[x ; y] .
\end{aligned}
$$

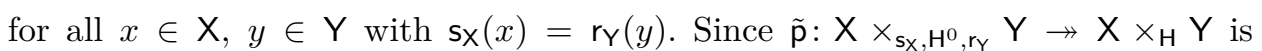
epic, we have $\mathrm{m}_{2} \circ\left(\operatorname{id}_{\left(\mathrm{X} \times{ }_{\mathrm{H}} \mathrm{Y}\right)} ; \mathrm{u}_{\mathrm{K}} \circ \mathrm{s}\right)=\mathrm{id}_{\left(\mathrm{X} \times{ }_{\mathrm{H}} \mathrm{Y}\right)}$. So $(5.2)$ holds. Therefore, the 
triple $\left(\mathrm{X} \times_{\mathrm{H}} \mathrm{Y} ; \mathrm{m}_{2} ; \mathrm{s}\right)$ is a right $\mathrm{K}$-action by Lemma 5.7. We have to prove that $\left(\mathrm{X} \times{ }_{\mathrm{H}} \mathrm{Y} ; \mathrm{m}_{1} ; \mathrm{m}_{2} ; \mathrm{s} ; \mathrm{r}\right)$ is a $\mathrm{G}, \mathrm{K}$-bibundle. We have

$$
\begin{aligned}
\mathrm{r}([x ; y] \cdot k) & =\mathrm{r}([x ; y \cdot k]) \\
& =\mathrm{r} \times(x) \\
& =\mathrm{r}([x ; y])
\end{aligned}
$$

for all $x \in \mathrm{X}, y \in \mathrm{Y}, k \in \mathrm{K}^{1}$ with $\mathrm{s}_{\mathbf{X}}(x)=\mathrm{r}_{\mathrm{Y}}(y)$ and $\mathrm{s}_{\mathrm{Y}}(y)=\mathrm{r}_{\mathrm{K}}(k)$. Since the

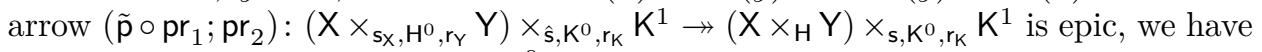
$\mathrm{r} \circ \mathrm{m}_{2}=\mathrm{r} \circ \mathrm{pr}_{1}$. So $\mathrm{r}: \mathrm{X} \times_{\mathrm{H}} \mathrm{Y} \rightarrow \mathrm{G}^{0}$ is $\mathrm{K}$-invariant. Also, we have

$$
\begin{aligned}
\mathrm{s}(g \cdot[x ; y]) & =\mathrm{s}([g \cdot x ; y]) \\
& =\mathrm{s}(y) \\
& =\mathrm{s}([x ; y]) .
\end{aligned}
$$

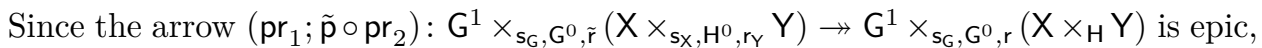
we have $\mathrm{s} \circ \mathrm{m}_{1}=\mathrm{s} \circ \mathrm{pr}_{2}$. So $\mathrm{s}: \mathrm{X} \times{ }_{\mathrm{H}} \mathrm{Y} \rightarrow \mathrm{K}^{0}$ is $\mathrm{G}$-invariant. We also have to show that the action maps $\mathrm{m}_{1}$ and $\mathrm{m}_{2}$ commutes. The arrow $\left(\left(\mathrm{pr}_{1} ; \tilde{\mathrm{p}} \circ \mathrm{pr}_{2}\right) \circ \mathrm{pr}_{1} ;\left(\tilde{\mathrm{p}} \circ \mathrm{pr}_{1} ; \mathrm{pr}_{2}\right) \circ \mathrm{pr}_{2}\right)$

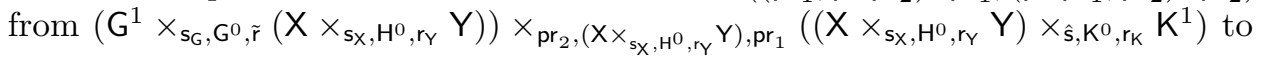
$\left(G^{1} \times_{s_{G}, G^{0}, r}\left(X \times{ }_{H} Y\right)\right) \times_{p_{2},},\left(X \times{ }_{H} Y\right), p_{1}\left(\left(X \times{ }_{H} Y\right) \times_{s, K^{0}, r_{K}} K^{1}\right)$ is epic because it is a composition of epics.

$\left(\left(\mathrm{pr}_{1} ; \tilde{\mathbf{p}} \circ \mathrm{pr}_{2}\right) \circ \mathrm{pr}_{1} ;\left(\tilde{\mathrm{p}} \circ \mathrm{pr}_{1} ; \mathrm{pr}_{2}\right) \circ \mathrm{pr}_{2}\right)=\left(\left(\mathrm{pr}_{1} ; \tilde{\mathrm{p}} \circ \mathrm{pr}_{2}\right) \circ \mathrm{pr}_{1} ; \mathrm{pr}_{2}\right) \circ\left(\mathrm{pr}_{1} ;\left(\tilde{\mathrm{p}} \circ \mathrm{pr}_{1} ; \mathrm{pr}_{2}\right) \circ \mathrm{pr}_{2}\right)$.

The right term of the composition is epic because it is a pull-back of the cover $\left(\tilde{\mathbf{p}} \circ \mathrm{pr}_{1} ; \mathrm{pr}_{2}\right)$. And the left term of the composition is epic because it is a pull-back of the cover $\left(\mathrm{pr}_{1} ; \tilde{\mathrm{p}} \circ \mathrm{pr}_{2}\right)$. So $\left(\left(\mathrm{pr}_{1} ; \tilde{\mathrm{p}} \circ \mathrm{pr}_{2}\right) \circ \mathrm{pr}_{1} ;\left(\tilde{\mathrm{p}} \circ \mathrm{pr}_{1} ; \mathrm{pr}_{2}\right) \circ \mathrm{pr}_{2}\right)$ is epic. We have

$$
\begin{aligned}
g \cdot([x ; y] \cdot k) & =g \cdot[x ; y \cdot k] \\
& =[g \cdot x ; y \cdot k] \\
& =[g \cdot x ; y \cdot k] \\
& =[g \cdot x ; y] \cdot k \\
& =(g \cdot[x ; y]) \cdot k
\end{aligned}
$$

for all $x \in \mathrm{X}, y \in \mathrm{Y}, g \in \mathrm{G}^{1}, k \in \mathrm{K}^{1}$ with $\mathrm{s}_{\mathbf{X}}(x)=\mathrm{r}_{\mathrm{Y}}(y), \mathrm{r}_{\mathbf{X}}(x)=\mathrm{s}_{\mathrm{G}}(g), \mathrm{s}_{\mathrm{Y}}(y)=\mathrm{r}_{\mathrm{K}}(k)$. Since the arrow $\left(\left(\mathrm{pr}_{1} ; \tilde{\mathrm{p}} \circ \mathrm{pr}_{2}\right) \circ \mathrm{pr}_{1} ;\left(\tilde{\mathrm{p}} \circ \mathrm{pr}_{1} ; \mathrm{pr}_{2}\right) \circ \mathrm{pr}_{2}\right)$ is epic, the action maps $\mathrm{m}_{1}$ and $\mathrm{m}_{2}$ commute: $\mathrm{m}_{1} \circ\left(\mathrm{pr}_{1} \circ \mathrm{pr}_{1} ; \mathrm{m}_{2} \circ \mathrm{pr}_{2}\right)=\mathrm{m}_{2} \circ\left(\mathrm{m}_{1} \circ \mathrm{pr}_{1} ; \mathrm{pr}_{2} \circ \mathrm{pr}_{2}\right)$. So $\left(\mathrm{X} \times_{\mathrm{H}} \mathrm{Y} ; \mathrm{m}_{1} ; \mathrm{m}_{2} ; \mathrm{s} ; \mathrm{r}\right)$ is a $\mathrm{G}, \mathrm{K}$-bibundle. The last step is to show that $\left(\mathrm{X} \times_{\mathrm{H}} \mathrm{Y} ; \mathrm{m}_{2} ; \mathrm{s}\right)$ is a principal K-bundle over $r: X \times_{H} \mathrm{Y} \rightarrow \mathrm{G}^{0}$.

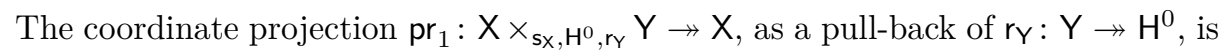
a cover. Since $r \circ \tilde{p}=\tilde{r}=r_{X} \circ p_{1}$ and the coordinate projection $\mathrm{pr}_{1}: X_{{ }_{1 X}, H^{0}, r_{Y} Y} Y \rightarrow X$

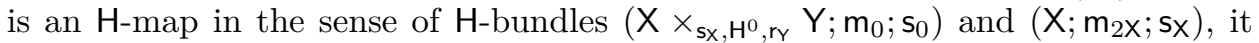
induces the arrow $r: X \times_{H} Y \rightarrow G^{0}$. Therefore, $r: X \times_{H} Y \rightarrow G^{0}$ is a cover by Corollary 5.53. Also, we need to show that the arrow

$$
\left(\mathrm{X} \times{ }_{\mathrm{H}} \mathrm{Y}\right) \times_{\mathrm{s}, \mathrm{K}^{0}, \mathrm{r}_{\mathrm{K}}} \mathrm{K}^{1} \stackrel{\left(\mathrm{pr}_{1} ; \mathrm{m}_{2}\right)}{\longrightarrow}\left(\mathrm{X} \times \times_{\mathrm{H}} \mathrm{Y}\right) \times_{\mathrm{r}, \mathrm{G}^{0}, \mathrm{r}}\left(\mathrm{X} \times{ }_{\mathrm{H}} \mathrm{Y}\right)
$$

is an isomorphism.

We know that $\left(X \times_{\mathbf{s X}_{X}, \mathrm{H}^{0}, \mathrm{rY}_{\mathrm{Y}}} \mathrm{Y} ; \hat{\mathrm{m}} ; \hat{\mathrm{s}}\right)$ is the pull-back of the right principal K-bundle $\left(\mathrm{Y} ; \mathrm{m}_{2 \mathrm{Y}} ; \mathrm{s}_{\mathrm{Y}}\right)$ over $\mathrm{r}_{\mathrm{Y}}: \mathrm{Y} \rightarrow \mathrm{H}^{0}$ along the arrow $\mathrm{s}_{\mathrm{X}}: \mathrm{X} \rightarrow \mathrm{H}^{0}$. Thus this $\mathrm{K}$-action

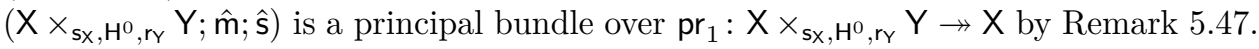
Therefore, we have the following isomorphism:

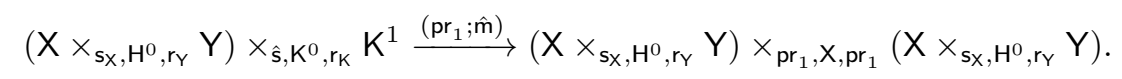




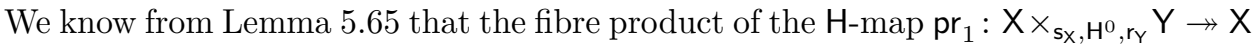
on itself is a principal $\mathrm{H}$-bundle over

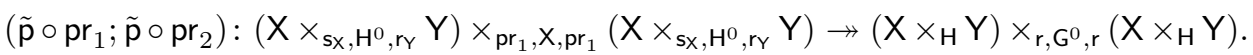

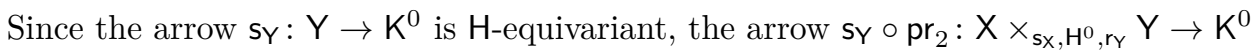
is $\mathrm{H}$-equivariant, too. Therefore, we can consider the pull-back of the $\mathrm{H}$-bundle

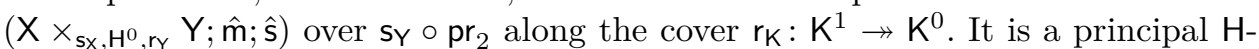

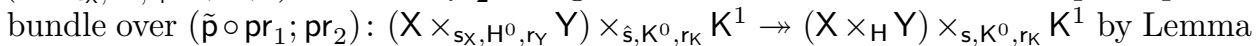
5.50 Since $\mathrm{m}_{2} \circ\left(\tilde{\mathrm{p}} \circ \mathrm{pr}_{1} ; \mathrm{pr}_{2}\right)=\tilde{\mathrm{p}} \circ \hat{\mathrm{m}}$, the arrow 6.1 is induced by the isomorphism in 6.2 Therefore, 6.1 is an isomorphism by Corollary 5.54 So $\left(\mathrm{Y} \circ \mathrm{X} ; \mathrm{m}_{1} ; \mathrm{m}_{2} ; \mathrm{s} ; \mathrm{r}\right)$ is a bibundle functor from $\mathrm{G}$ to $\mathrm{K}$.

Corollary 6.16. Under Assumptions 2.14, 2.15, 2.18, 2.19 and 5.63, we can compose bibundle actors as in Proposition 6.15 .

Proof. The proof is almost the same. We just use the cover $\mathrm{p}: \mathbf{X} \rightarrow \mathbf{Z}$ instead of the cover $r_{X}: X \rightarrow G^{0}$. One of the differences is that in this case we have to show that the anchor map $\mathrm{s}: \mathrm{X} \times_{\mathrm{G}} \mathrm{Y} \rightarrow \mathrm{K}^{0}$ is a cover. We have $\mathrm{s} \circ \tilde{\mathrm{p}}=\mathrm{sY}_{\mathrm{Y}} \circ \mathrm{pr}_{2}$. The coordinate projection $\mathrm{pr}_{2}: X \times_{s_{X}, H^{0}, r_{Y}} \mathrm{Y} \rightarrow \mathrm{Y}$ is a cover because it is a pull-back of the cover $s_{X}: X \rightarrow H^{0}$. Thus $s_{Y} \circ p_{2}$ is a cover by Assumption 2.14. Since

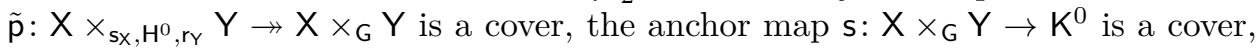
too by Assumption 2.19

Also, there is one more important difference from the proof of Proposition 6.15 . We have to find an orbit space projection of the K-action on $X \times_{H} Y$. For this we must define the right $\mathrm{H}$-action on $\mathrm{Z}$ elementwise by $[y] \cdot h=\left[h^{-1} \cdot y\right]$, and then consider the fibre product of $\mathrm{H}$-actions on $\mathrm{X}$ and $\mathrm{Z}$ and get the $\mathrm{H}$-action on $\mathrm{X} \times_{\mathbf{s x}_{\mathrm{X}} \mathrm{H}^{0}, \alpha} \mathbf{Z}$ defined elementwise by $(x ;[y]) \cdot h=\left(x \cdot h ;\left[h^{-1} \cdot y\right]\right)$. This action is basic because the coordinate projection $\mathrm{pr}_{1}: \mathrm{X} \times_{\mathrm{sX}_{\mathrm{X}}, \mathrm{H}^{0}, \alpha} \mathrm{Z} \rightarrow \mathrm{X}$ is an $\mathrm{H}$-map, and then, similarly as in case of bibundle functors, we can deduce that the orbit space projection of the $\mathrm{K}$-action on $\mathrm{X} \times_{\mathrm{H}} \mathrm{Y}$ is a cover from $\mathrm{X} \times_{\mathrm{H}} \mathrm{Y}$ to $\mathrm{X} \times_{\mathrm{H}} \mathrm{Z}$ induced by the orbit space

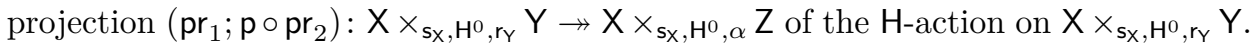
Here $\alpha: Z \rightarrow \mathrm{H}^{0}$ is the unique arrow such that $\alpha \circ \mathrm{p}=\mathrm{r}_{\mathrm{Y}}$.

Remark 6.17. Consider the following assumption. If $f \circ g$ is a partial cover and $g$ is a cover then $f$ is a partial cover. This assumption holds in most examples of categories with partial covers which are discussed in this thesis. Under this assumption we can compose partial bibundle actors. We have to check that the anchor map s: $\mathrm{X} \times{ }_{\mathrm{G}} \mathrm{Y} \rightarrow \mathrm{K}^{0}$ is a partial cover. We have that $\mathrm{s} \circ \tilde{\mathrm{p}}$ is a partial cover and $\tilde{p}$ is a cover. Therefore, $s: X \times{ }_{G} Y \rightarrow K^{0}$ is a partial cover by assumption above.

Remark 6.18. The composition of (partial) bibundle actors is a naturally associative. Consider three composable (partial) bibundle actors $\left(X ; m_{1 X} ; m_{2 X} ; s_{X} ; r_{X}\right)$, $\left(\mathrm{Y} ; \mathrm{m}_{1 \mathrm{Y}} ; \mathrm{m}_{2 \mathrm{Y}} ; \mathrm{s}_{\mathrm{Y}} ; \mathrm{r}_{\mathrm{Y}}\right)$ and $\left(\mathrm{Z} ; \mathrm{m}_{1 Z} ; \mathrm{m}_{2 \mathrm{Z}} ; \mathrm{s}_{\mathrm{Z}} ; \mathrm{r}_{\mathrm{Z}}\right)$ from $\mathrm{G}$ to $\mathrm{H}$, from $\mathrm{H}$ to $\mathrm{K}$ and from $\mathrm{K}$ to $L$, respectively. The object $Y \times_{s_{Y}, K^{0}, r_{Z}} Z$ with obvious actions of $H$ and $K$ on it is a (partial) bibundle actor from $\mathrm{H}$ to $\mathrm{K}$. Hence we can consider the composition of this (partial) bibundle actor and $\left(X ; m_{1 X} ; m_{2 X} ; s_{X} ; r_{X}\right)$. We get a (partial) bibundle actor from $G$ to $K$ defined by the object $X \times_{H}\left(Y \times_{s_{Y}, K^{0},{ }_{r Z}} Z\right)$ and by suitable $G$ and $\mathrm{K}$ actions on it. We know from the proof of Corollary 6.16 that an orbit space of

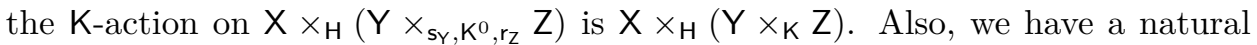
isomorphism $X \times_{H}\left(Y \times_{s_{Y}, K^{0}, r_{Z}} Z\right) \cong\left(X \times_{H} Y\right) \times_{s^{\prime}, K^{0}, r_{Z}} Z$ induced by the canonical

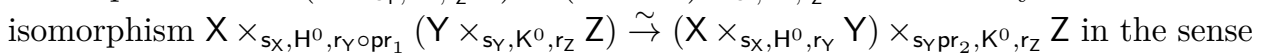
of the $\mathrm{H}$-actions defined elementwise by $(x ;(y ; z)) \cdot h=\left(x \cdot h ;\left(h^{-1} \cdot y ; z\right)\right)$ and $\left.((x ; y) ; z) \cdot h=\left(x \cdot h ; h^{-1} \cdot y\right) ; z\right)$, respectively. An orbit space of the K-action on

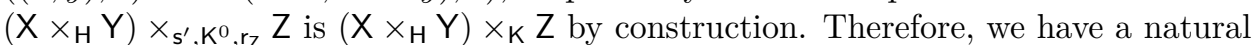
isomorphism $X \times_{H}\left(Y \times_{K} Z\right) \cong\left(X \times_{H} Y\right) \times_{K} Z$. 
6.4. Bibundle equivalence. In this subsections we define equivalence of groupoids. It is the same as Morita equivalence. Let $\mathrm{G}=\left(\mathrm{G}^{0}, \mathrm{G}^{1}, \mathrm{r}_{\mathrm{G}}, \mathrm{s}_{\mathrm{G}}, \mathrm{m}_{\mathrm{G}}\right)$ and $\mathrm{H}=$ $\left(\mathrm{H}^{0}, \mathrm{H}^{1}, \mathrm{r}_{\mathrm{H}}, \mathrm{s}_{\mathrm{H}}, \mathrm{m}_{\mathrm{H}}\right)$ be groupoids in the category $\left(\mathcal{C}, \mathcal{F}_{p}\right)$ with partial covers.

Definition 6.19. A (partial) bibundle equivalence from $\mathrm{G}$ to $\mathrm{H}$ is a $\mathrm{G}, \mathrm{H}$-bibundle $\left(\mathrm{X} ; \mathrm{m}_{1 \mathrm{X}} ; \mathrm{m}_{2 \mathrm{X}} ; \mathrm{s}_{\mathrm{X}} ; \mathrm{r}_{\mathrm{X}}\right)$, where the right $\mathrm{H}$-bundle $\left(\mathrm{X} ; \mathrm{m}_{2 \mathrm{X}} ; \mathrm{s}_{\mathrm{X}}\right)$ is (partially) principal over $r_{X}: X \rightarrow G^{0}$ and the left $G$-bundle $\left(X ; m_{1 X} ; r_{X}\right)$ is (partially) principal over $\mathrm{s}_{\mathrm{X}}: \mathrm{X} \rightarrow \mathrm{G}^{0}$. Then we call the groupoids $\mathrm{G}$ and $\mathrm{H}$ equivalent.

Remark 6.20. A bibundle equivalence from $\mathrm{G}$ to $\mathrm{H}$ is also a bibundle actor and a bibundle functor. It has all required properties of being such kinds of generalised morphisms.

Lemma 6.21. Under Assumptions 2.15, 2.18 and 5.66, equivalence of groupoids as defined in Definition 6.19 is reflexive, symmetric and transitive.

Proof. Equivalence is reflexive because for any groupoid $G=\left(G^{0}, G^{1}, r, s, m\right)$ the left and right translation actions define a bibundle equivalence $\left(\mathrm{G}^{1} ; \mathrm{m} ; \mathrm{m} ; \mathrm{s} ; \mathrm{r}\right) \equiv \mathbb{G}^{1}$ from $\mathrm{G}$ to $\mathrm{G}$ because of the properties (2), (3) and (4) in Definition 3.1.

Equivalence is symmetric, too, because we can use Lemma 5.18 and change the left $\mathrm{G}$-action and right $\mathrm{H}$-action with a suitable right $\mathrm{G}$-action and left $\mathrm{H}$ action, respectively, and they will give a bibundle equivalence from $\mathrm{H}$ to $\mathrm{G}$. Thus if $\left(X ; m_{1 X} ; m_{2 X} ; s_{X} ; r_{X}\right) \equiv \mathbb{X}$ is a bibundle equivalence from $G$ to $H$ then $\left(X ; m_{2 X} \circ\right.$ $\left.\left(\mathrm{pr}_{2} ; \mathrm{i}_{\mathrm{H}} \circ \mathrm{pr}_{1}\right) ; \mathrm{m}_{1 \mathrm{X}} \circ\left(\mathrm{i}_{\mathrm{G}} \circ \mathrm{pr}_{2} ; \mathrm{pr}_{1}\right) ; \mathrm{rX}_{\mathrm{X}} ; \mathrm{sX}_{\mathrm{X}}\right) \equiv \mathbb{X}^{-1}$ is a bibundle equivalence from $\mathrm{H}$ to $\mathrm{G}$.

If $\left(X ; m_{1 X} ; m_{2 X} ; s_{X} ; r_{X}\right) \equiv \mathbb{X}$ and $\left(Y ; m_{1 Y} ; m_{2 Y} ; s_{Y} ; r_{Y}\right) \equiv \mathbb{Y}$ are bibundle equivalences from $\mathrm{G}$ to $\mathrm{H}$ and from $\mathrm{H}$ to $\mathrm{R}$, respectively, then their composition, as a bibundle functors, is a bibundle functor from $\mathrm{G}$ to $\mathrm{R}$. We are going to show that this composition $\left(\mathrm{X} \times_{\mathrm{H}} \mathrm{Y} ; \mathrm{m}_{1} ; \mathrm{m}_{2} ; \mathrm{s} ; \mathrm{r}\right) \equiv \mathbb{Y} \circ \mathbb{X}$, described in the proof of Propesition 6.15. is a bibundle equivalence from $G$ to $R$. We need to show that the left G-bundle $\left(\mathrm{X} \times{ }_{\mathrm{H}} \mathrm{Y} ; \mathrm{m}_{1} ; \mathrm{r}\right)$ over $\mathrm{s}: \mathrm{X} \times_{\mathrm{H}} \mathrm{Y} \rightarrow \mathrm{R}^{0}$ is principal. We have an analogous situation as in the case of the right principal R-bundle $\left(X \times_{H} Y ; m_{2} ; s\right)$ over $r: X \times_{H} Y \rightarrow G^{0}$. The anchor map $\mathrm{s}: \mathrm{X} \times_{\mathrm{H}} \mathrm{Y} \rightarrow \mathrm{R}^{0}$ is a cover because it is induced by the cover

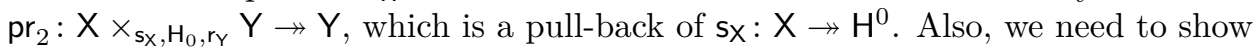
that the arrow

$$
\mathrm{G}^{1} \times_{\mathrm{s}_{\mathrm{G}}, \mathrm{G}^{0}, \mathrm{r}}\left(\mathrm{X} \times \times_{\mathrm{H}} \mathrm{Y}\right) \stackrel{\left(\mathrm{m}_{1} ; \mathrm{pr}_{2}\right)}{\longrightarrow}\left(\mathrm{X} \times \times_{\mathrm{H}} \mathrm{Y}\right) \times_{\mathrm{s}, \mathrm{R}^{0}, \mathrm{~s}}\left(\mathrm{X} \times \times_{\mathrm{H}} \mathrm{Y}\right)
$$

is an isomorphism. That is right because it is induced by the following isomorphism:

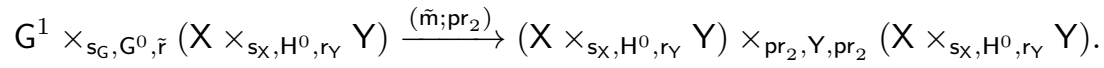

This proof shows that we can compose bibundle equivalences like bibundle actors and bibundle functors.

Lemma 6.22. Assume Assumptions 2.15, 2.18 and 5.66. The bibundle equivalence $\left(\mathrm{G}^{1} ; \mathrm{m} ; \mathrm{m} ; \mathrm{s} ; \mathrm{r}\right) \equiv \mathbb{G}^{1}$ from a groupoid $\mathrm{G}$ to itself is an identity in the sense of the composition of bibundle actors, and each bibundle equivalence is invertible in the same sense.

Proof. Let $\left(\mathrm{X} ; \mathrm{m}_{1 \mathrm{X}} ; \mathrm{m}_{2 \mathrm{X}} ; \mathrm{s}_{\mathbf{X}} ; \mathrm{r}_{\mathbf{X}}\right) \equiv \mathbb{X}$ be a bibundle equivalence from $\mathrm{G}$ to $\mathrm{H}$, and let $\left(\mathrm{G} \times{ }_{\mathrm{G}} \mathrm{X} ; \mathrm{m}_{1} ; \mathrm{m}_{2} ; \mathrm{s} ; \mathrm{r}\right) \equiv \mathbb{X} \circ \mathbb{G}^{1}$ be a composition of $\left(\mathrm{G}^{1} ; \mathrm{m} ; \mathrm{m} ; \mathrm{s} ; \mathrm{r}\right) \equiv \mathbb{G}^{1}$ and $\left(X ; m_{1 X} ; m_{2 X} ; s_{X} ; r_{X}\right) \equiv \mathbb{X}$. The object $G^{1} \times_{G} X$ is an orbit space of the G-action on

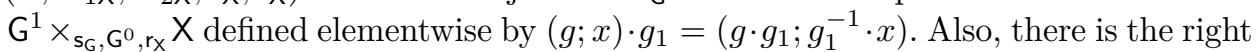

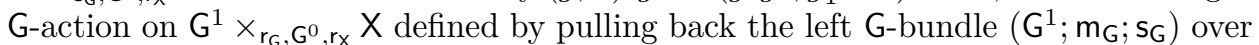
the range map $r_{G}: G^{1} \rightarrow G^{0}$ along $r_{X}: X \rightarrow G^{0}$. This action is defined elementwise 
by $(g ; x) \cdot g_{1}=\left(g \cdot g_{1} ; x\right)$. The coodrinate projection $\mathrm{pr}_{2}: \mathrm{G}^{1} \times_{\mathrm{r}_{\mathrm{G}}, \mathrm{G}^{0}, \mathrm{r}_{\mathrm{X}}} \mathrm{X} \rightarrow \mathrm{X}$ is an orbit space projection of this action by Lemma 5.50. The isomorphism

$$
\left(\mathrm{pr}_{1} ; \mathrm{m}_{1 \mathrm{X}}\right): \mathrm{G}^{1} \times_{\mathrm{s}_{\mathrm{G}}, \mathrm{G}^{0}, \mathrm{r}_{\mathrm{X}} \mathrm{X}} \mathrm{X} \mathrm{G}^{1} \times_{\mathrm{r}_{\mathrm{G}}, \mathrm{G}^{0}, \mathrm{r}_{\mathrm{X}}} \mathrm{X}
$$

is a G-map because

$$
\begin{aligned}
\left(\mathrm{pr}_{1} ; \mathrm{m}_{1 \mathrm{X}}\right)\left((g ; x) \cdot g_{1}\right) & =\left(\mathrm{pr}_{1} ; \mathrm{m}_{1 \mathrm{X}}\right)\left(g \cdot g_{1} ; g_{1}^{-1} \cdot x\right) \\
& =\left(g \cdot g_{1} ; g \cdot g_{1} \cdot g_{1}^{-1} \cdot x\right) \\
& =\left(g \cdot g_{1} ; g \cdot x\right) \\
& =(g ; g \cdot x) \cdot g_{1} \\
& =\left(\mathrm{pr}_{1} ; \mathrm{m}_{1 \mathrm{X}}\right)(g ; x) \cdot g_{1} .
\end{aligned}
$$

Also, it clearly commutes with anchor maps, and therefore, it induces an isomorphism $\varphi: \mathrm{G}^{1} \times{ }_{\mathrm{G}} \mathrm{X} \stackrel{\sim}{\rightarrow} \mathrm{X}$ such that $\varphi \circ \tilde{\mathrm{p}}=\mathrm{pr}_{2} \circ\left(\mathrm{pr}_{1} ; \mathrm{m}_{1 \mathrm{X}}\right)=\mathrm{m}_{1 \mathrm{X}}$. Elementwise $\varphi([g ; x])=g \cdot x$. This isomorphism is a $\mathrm{G}, \mathrm{H}$-map because

$$
\begin{aligned}
\varphi\left(g_{1} \cdot[g ; x]\right) & =\varphi\left(\left[g_{1} \cdot g ; x\right]\right) \\
& =g_{1} \cdot g \cdot x \\
& =g_{1} \cdot(\varphi([g ; x]),
\end{aligned}
$$

and

$$
\begin{aligned}
\varphi([g ; x] \cdot h) & =\varphi([g ; x \cdot h]) \\
& =g \cdot x \cdot h \\
& =(\varphi([g ; x]) \cdot h .
\end{aligned}
$$

Therefore, it gives the isomorphism between $\mathrm{G}, \mathrm{H}$-bibundles $\mathbb{X}$ and $\mathbb{X} \circ \mathbb{G}^{1}$. Analogously, we can construct a $\mathrm{G}, \mathrm{H}$-isomorphism between $\mathbb{X}$ and $\mathbb{H}^{1} \circ \mathbb{X}$

Consider the composition of the bibundle equivalence $\left(X ; m_{1 X} ; m_{2 X} ; s_{X} ; r_{X}\right)$ from $G$ to $\mathrm{H}$ and the bibundle equivalence $\left(\mathrm{X} ; \mathrm{m}_{2 \mathrm{X}} \circ\left(\mathrm{pr}_{2} ; \mathrm{i}_{\mathrm{H}} \circ \mathrm{pr}_{1}\right) ; \mathrm{m}_{1 \mathrm{X}} \circ\left(\mathrm{i}_{\mathrm{G}} \circ \mathrm{pr}_{2} ; \mathrm{pr}_{1}\right) ; \mathrm{rX}_{\mathrm{X}} ; \mathrm{sX}\right)$

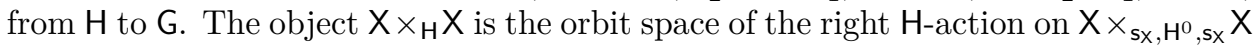
defined elementwise by $\left(x ; x_{1}\right) \cdot h=\left(x \cdot h ; h^{-1} \cdot x_{1}\right)=\left(x \cdot h ; x_{1} \cdot h\right)$. Also, there

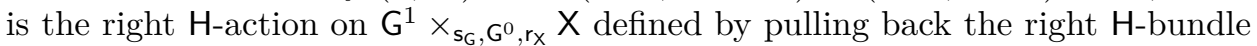
$\left(X ; m_{2 X} ; s_{X}\right)$ over $r_{X}: X \rightarrow G^{0}$ along the source map $s_{G}: G^{1} \rightarrow G^{0}$. This action is defined elementwise by $(g ; x) \cdot h=(g ; x \cdot h)$. This action is a principal bundle over $\mathrm{pr}_{1}: \mathrm{G}^{1} \times_{\mathrm{s}_{\mathrm{G}}, \mathrm{G}^{0}, \mathrm{rX}} \mathrm{X} \rightarrow \mathrm{G}^{1}$ by Lemma 5.50 The isomorphism

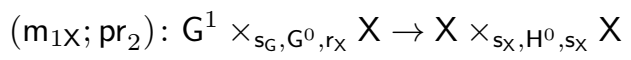

is an $\mathrm{H}$-map because

$$
\begin{aligned}
\left(\mathrm{m}_{1 \mathrm{x}} ; \mathrm{pr}_{2}\right)((g ; x) \cdot h) & =\left(\mathrm{m}_{1 \mathrm{x}} ; \mathrm{pr}_{2}\right)(g ; x \cdot h) \\
& =(g \cdot(x \cdot h) ; x \cdot h) \\
& =((g \cdot x) \cdot h ; x \cdot h) \\
& =(g \cdot x ; x) \cdot h \\
& =\left(\mathrm{m}_{1 \mathrm{x}} ; \mathrm{pr}_{2}\right)(g ; x) \cdot h .
\end{aligned}
$$

Also, it clearly commutes with anchor maps, and therefore, it induces an isomorphism $\psi: \mathrm{G}^{1} \stackrel{\sim}{\rightarrow} \mathrm{X} \times{ }_{\mathrm{G}} \mathrm{X}$ such that $\psi \circ \mathrm{pr}_{1}=\tilde{\mathrm{p}} \circ\left(\mathrm{m}_{1 \mathrm{X}} ; \mathrm{pr}_{2}\right)$. Elementwise $\psi(g)=[g \cdot x ; x]$ This isomorphism is a G, G-map in the sense of G, G-bibundles $\left(G^{1} ; m ; m ; s ; r\right)$ and the composition of the bibundle equivalence $\left(X ; m_{1 X} ; m_{2 X} ; s_{X} ; r_{X}\right)$ from $G$ to $H$ and the bibundle equivalence $\left(X ; \mathrm{m}_{2 \mathrm{X}} \circ\left(\mathrm{pr}_{2} ; \mathrm{i}_{\mathrm{H}} \circ \mathrm{pr}_{1}\right) ; \mathrm{m}_{1 \mathrm{X}} \circ\left(\mathrm{i}_{\mathrm{G}} \circ \mathrm{pr}_{2} ; \mathrm{pr}_{1}\right) ; \mathrm{rX}_{\mathrm{X}} ; \mathrm{sX}\right)$ from $\mathrm{H}$ 
to $G$ because

$$
\begin{aligned}
\psi\left(g_{1} \cdot g\right) & =\left[\left(g_{1} \cdot g\right) \cdot x ; x\right] \\
& =\left[g_{1} \cdot(g \cdot x) ; x\right] \\
& =g_{1} \cdot[g \cdot x ; x] \\
& =g_{1} \cdot \psi(g),
\end{aligned}
$$

and

$$
\begin{aligned}
\psi\left(g \cdot g_{1}\right) & =\left[\left(g \cdot g_{1}\right) \cdot\left(g_{1}^{-1} \cdot x\right) ;\left(g_{1}^{-1} \cdot x\right)\right] \\
& =\left[g \cdot x ; g_{1}^{-1} \cdot x\right] \\
& =[g \cdot x ; x] \cdot g_{1}=\psi(g) \cdot g_{1} .
\end{aligned}
$$

So the composition of the bibundle equivalence $\left(X ; m_{1 X} ; m_{2 X} ; s_{X} ; r_{X}\right)$ from $G$ to $H$ and the bibundle equivalence $\left(X ; \mathrm{m}_{2 \mathrm{X}} \circ\left(\mathrm{pr}_{2} ; \mathrm{i}_{\mathrm{H}} \circ \mathrm{pr}_{1}\right) ; \mathrm{m}_{1 \mathrm{X}} \circ\left(\mathrm{i}_{\mathrm{G}} \circ \mathrm{pr}_{2} ; \mathrm{pr}_{1}\right) ; \mathrm{r}_{\mathrm{X}} ; \mathrm{sX}\right)$ from $H$ to $G$ gives a bibundle equivalence isomorphic to $\left(G^{1} ; m ; m ; s ; r\right)$. Therefore, the bibundle equivalence $\left(\mathrm{X} ; \mathrm{m}_{2 \mathrm{X}} \circ\left(\mathrm{pr}_{2} ; \mathrm{i}_{\mathrm{H}} \circ \mathrm{pr}_{1}\right) ; \mathrm{m}_{1 \mathrm{X}} \circ\left(\mathrm{i}_{\mathrm{G}} \circ \mathrm{pr}_{2} ; \mathrm{pr}_{1}\right) ; \mathrm{r}_{\mathrm{X}} ; \mathrm{sX}\right)$ is an inverse of the bibundle equivalence $\left(X ; m_{1 X} ; m_{2 X} ; s_{X} ; r_{X}\right)$.

\section{Generalised groupoid aCtions}

In this section, we discuss generalised groupoid actions. Let $\mathrm{G}, \mathrm{H}, \mathrm{L}, \mathrm{K}$ and $\mathrm{R}$ be groupoids in the category $\left(\mathcal{C}, \mathcal{F}_{p}\right)$ with partial covers.

Definition 7.1. We say that the groupoid $\mathrm{H}$ acts on the groupoid $\mathrm{G}$ by a groupoid fibration $\mathrm{F}: \mathrm{L} \rightarrow \mathrm{H}$ if the fibre of $\mathrm{F}$ is $\mathrm{G}$. We call this a generalised groupoid action.

Proposition 7.2. Under Assumptions 2.14, 2.15, 2.18, 2.19 and 5.63, a generalised groupoid action can be transformed along a bibundle equivalence. That is, if $\mathrm{H}$ acts on $\mathrm{G}$ by a groupoid fibration $\mathrm{F}: \mathrm{L} \rightarrow \mathrm{H}$ and if $\mathrm{G}$ and $\mathrm{K}$ are equivalent, then we can construct an action of $\mathrm{H}$ on $\mathrm{K}$. In other words, we can construct a groupoid fibration $\mathrm{E}: \mathrm{R} \rightarrow \mathrm{H}$ with fibre $\mathrm{K}$ such that $\mathrm{R}$ and $\mathrm{L}$ are equivalent.

Proof. The first step of the proof is to construct the arrows of the groupoid R. Let $\left(X ; m_{1 X} ; m_{2 X} ; s_{X} ; r_{X}\right)$ be a bibundle equivalence from $G$ to $K$. Since the range

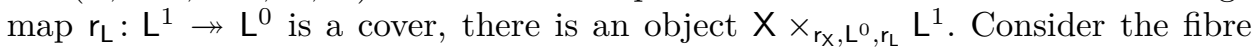

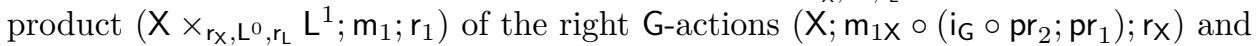
$\left(\mathrm{L}^{1}, \mathrm{~m}_{\mathrm{L}} \circ\left(\mathrm{i}_{\mathrm{L}} \circ \mathrm{pr}_{1} \circ \mathrm{pr}_{2} ; \mathrm{pr}_{1}\right), \mathrm{s}_{\mathrm{L}}\right)$ (see Example 5.25). This action is defined elementwise by

$$
(x ; l) \cdot g=\left(g^{-1} \cdot x ; g^{-1} \cdot l\right)
$$

for all $x \in \mathrm{X}, g \in \mathrm{G}^{1}, l \in \mathrm{L}^{1}$ with $\mathrm{r}_{\mathrm{X}}(x)=\mathrm{r}_{\mathrm{L}}(l)=\mathrm{r}_{\mathrm{G}}(g)$. We know that this action is principal, too, by Assumption 5.63. Therefore, there is an orbit space projection

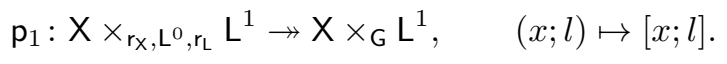

The arrow $\mathrm{s}_{\mathrm{L}} \circ \mathrm{pr}_{2}: \mathrm{X} \times{ }_{\mathrm{r}_{\mathrm{x}}, \mathrm{L}^{0}, \mathrm{r}_{\mathrm{L}}} \mathrm{L}^{1} \rightarrow \mathrm{L}^{0}$ is G-invariant because

$$
\begin{aligned}
\mathrm{s}_{\mathrm{L}}\left(\operatorname{pr}_{2}((x ; l) \cdot g)\right. & =\mathrm{s}_{\mathrm{L}}\left(\operatorname{pr}_{2}\left(g^{-1} \cdot x ; g^{-1} \cdot l\right)\right) \\
& =\mathrm{s}_{\mathrm{L}}\left(g^{-1} \cdot l\right) \\
& =\mathrm{s}_{\mathrm{L}}(l)=\mathrm{s}_{\mathrm{L}}\left(\operatorname{pr}_{2}(x ; l)\right) .
\end{aligned}
$$

Therefore, we can consider the pull-back of the G-bundle $\left(X \times_{r_{X}, L^{0}, r_{L}} L^{1} ; m_{1} ; r_{1}\right)$ over

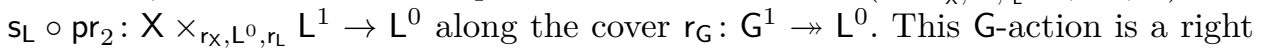
principal G-bundle over

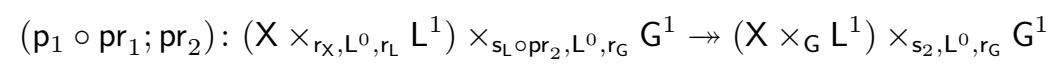

by Lemma 5.50 where $\mathrm{s}_{2}: \mathrm{X} \times{ }_{\mathrm{G}} \mathrm{L}^{1} \rightarrow \mathrm{L}^{0}$ is the unique arrow such that $\mathrm{s}_{2} \circ \mathrm{p}_{1}=\mathrm{s}_{\mathrm{L}} \circ \mathrm{pr}_{2}$. Elementwise $\mathrm{s}_{2}([x ; l])=\mathrm{s}_{\mathrm{L}}(l)$. Let us show that $\mathrm{m}_{2}^{\prime}$ is $\mathrm{G}$-invariant, where $\mathrm{m}_{2}^{\prime}$ is the 
action map of the pull-back of the right G-bundle $\left(\mathrm{L}^{1} ; \mathrm{m}_{\mathrm{L}} \circ\left(\mathrm{pr}_{1} ; \mathrm{pr}_{1} \circ \mathrm{pr}_{2}\right) ; \mathrm{s}_{\mathrm{L}}\right)$ over $r_{L}: L^{1} \rightarrow L^{0}$ along the cover $r_{X}: X \rightarrow L^{0}$. This action map is defined elementwise by $(x ; l) \cdot g=(x ; l \cdot g)$. We have

$$
\begin{aligned}
((x ; l) \cdot g) \cdot g_{1} & =\left(g^{-1} \cdot x ; g^{-1} \cdot l\right) \cdot g_{1} \\
& =\left(g^{-1} \cdot x ;\left(g^{-1} \cdot l\right) \cdot g_{1}\right) \\
& =\left(g^{-1} \cdot x ; g^{-1} \cdot\left(l \cdot g_{1}\right)\right) \\
& =\left(x ; l \cdot g_{1}\right) \cdot g \\
& =\left((x ; l) \cdot g_{1}\right) \cdot g
\end{aligned}
$$

for all $x \in \mathrm{X}, g, g_{1} \in \mathrm{G}^{1}, l \in \mathrm{L}^{1}$ with $\mathrm{rX}_{\mathbf{X}}(x)=\mathrm{r}_{\mathrm{L}}(l)=\mathrm{r}_{\mathrm{G}}(g)$ and $\mathrm{s}_{\mathrm{L}}(l)=\mathrm{r}_{\mathrm{G}}\left(g_{1}\right)$. This shows that the action map $\mathrm{m}_{2}^{\prime}$ is $\mathrm{G}$-invariant. Therefore, it induces a unique arrow

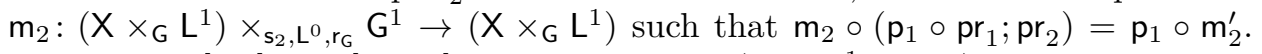
Elementwise $[x ; l] \cdot g=[x ; l \cdot g]$. Let us show that $\left(\mathrm{X} \times_{\mathrm{G}} \mathrm{L}^{1} ; \mathrm{m}_{2} ; \mathrm{s}_{2}\right)$ is a right $\mathrm{G}$-action. We have

$$
\begin{aligned}
\mathrm{s}_{2}([x ; l] \cdot g) & =\mathrm{s}_{2}([x ; l \cdot g]) \\
& =\mathrm{s}_{\mathrm{L}}(l \cdot g) \\
& =\mathrm{s}_{\mathrm{L}}(g) .
\end{aligned}
$$

So the property (1) holds. Also

$$
\begin{aligned}
([x ; l] \cdot g) \cdot g_{1} & =([x ; l \cdot g]) \cdot g_{1} \\
& =\left[x ;(l \cdot g) \cdot g_{1}\right] \\
& =\left[x ; l \cdot\left(g \cdot g_{1}\right)\right] \\
& =[x ; l] \cdot\left(g \cdot g_{1}\right) .
\end{aligned}
$$

That gives $(2)$. And $[x ; l] \cdot 1_{\mathrm{s}_{2}([x ; l])}=\left[x ; l \cdot 1_{\mathrm{s}_{\mathrm{L}}(l)}\right]=[x ; l]$. So $\left(\mathrm{X} \times{ }_{\mathrm{G}} \mathrm{L}^{1} ; \mathrm{m}_{2} ; \mathrm{s}_{2}\right)$ is a right G-action.

Consider the fibre product $\left(\left(X \times_{G} L^{1}\right) \times_{s_{2}, L^{0}, r_{X}} X ; m_{3} ; r_{3}\right)$ of the right $G$-actions $\left(X \times_{G} L^{1} ; m_{2} ; s_{2}\right)$ and $\left(X ; m_{1 X} \circ\left(i_{G} \circ p_{2} ; p_{1}\right) ; r_{X}\right)$. This action is defined elementwise by

$$
\left([x ; l] ; x_{1}\right) \cdot g=\left([x ; l \cdot g] ; g^{-1} \cdot x_{1}\right)
$$

for all $x, x_{1} \in \mathrm{X}, g \in \mathrm{G}^{1}, l \in \mathrm{L}^{1}$ with $\mathrm{r}_{\mathbf{X}}(x)=\mathrm{r}_{\mathrm{L}}(l)$ and $\mathrm{r}_{\mathbf{X}}\left(x_{1}\right)=\mathrm{s}_{\mathrm{L}}(l)=\mathrm{r}_{\mathrm{G}}(g)$. We know that the coordinate projection $\mathrm{pr}_{2}:\left(\mathrm{X} \times_{\mathrm{G}} \mathrm{L}^{1}\right) \times_{\mathrm{s}_{2}, \mathrm{~L}^{0}, r_{\mathrm{X}}} \mathrm{X} \rightarrow \mathrm{X}$ is a G-map. Therefore, $\left(\left(X \times_{G} L^{1}\right) \times_{s_{2}, L^{0}, r_{X}} X ; m_{3} ; r_{3}\right)$ is a principal G-bundle over some cover

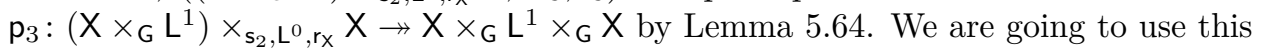
object $X \times_{G} L^{1} \times_{G} X$ as arrows of the groupoid $R$. So let

$$
\mathrm{R}^{1}=\mathrm{X} \times \times_{\mathrm{G}} \mathrm{L}^{1} \times_{\mathrm{G}} \mathrm{X} .
$$

The next step is to define the groupoid structure on $R$. It is obvious that $\mathrm{R}^{0}=\mathrm{K}^{0}$ because $\mathrm{K}$ must be a fibre of the groupoid fibration $\mathrm{E}: \mathrm{R} \rightarrow \mathrm{H}$. The $\mathrm{G}$-map

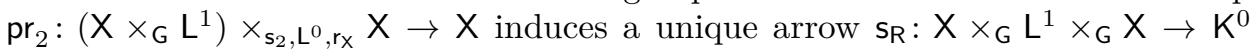
such that $\mathrm{s}_{\mathrm{R}} \circ \mathrm{p}_{3}=\mathrm{sx}_{\mathrm{X}} \circ \mathrm{pr}_{2}$. Elementwise $\mathrm{s}_{\mathrm{R}}\left(\left[[x ; l] ; x_{1}\right]\right)=\mathrm{s}_{\mathrm{X}}\left(x_{1}\right)$. The G-map

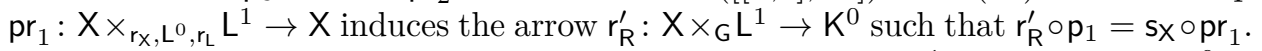
Elementwise $r_{R}^{\prime}([x ; l])=s_{X}(x)$. The arrow $r_{R}^{\prime} \circ p r_{1}:\left(X \times{ }_{G} L^{1}\right) \times_{s_{2}, L^{0}, r_{X}} \mathrm{X} \rightarrow \mathrm{K}^{0}$ is G-invariant in the sense of the G-action $\left(\left(X \times_{G} L^{1}\right) \times_{s_{2}, L^{0}, r_{X}} X ; m_{3} ; r_{3}\right)$ because

$$
\begin{aligned}
\mathrm{r}_{\mathrm{R}}^{\prime}\left(\operatorname{pr}_{1}\left(\left([x ; l] ; x_{1}\right) \cdot g\right)\right) & =\mathrm{r}_{\mathrm{R}}^{\prime}\left(\operatorname{pr}_{1}\left([x ; l \cdot g] ; g^{-1} \cdot x_{1}\right)\right) \\
& =\mathrm{r}_{\mathrm{R}}^{\prime}([x ; l \cdot g]) \\
& =\mathrm{sX}_{\mathbf{X}}(x) \\
& =\mathrm{r}_{\mathrm{R}}^{\prime}([x ; l]) \\
& =\mathrm{r}_{\mathrm{R}}^{\prime}\left(\operatorname{pr}_{1}\left([x ; l] ; x_{1}\right)\right)
\end{aligned}
$$


for all $x, x_{1} \in \mathrm{X}, g \in \mathrm{G}^{1}, l \in \mathrm{L}^{1}$ with $\mathrm{r}_{\mathrm{X}}(x)=\mathrm{r}_{\mathrm{L}}(l)$ and $\mathrm{r}_{\mathrm{X}}\left(x_{1}\right)=\mathrm{s}_{\mathrm{L}}(l)=\mathrm{r}_{\mathrm{G}}(g)$. Therefore, there is a unique arrow $\mathrm{r}_{\mathrm{R}}: \mathrm{X} \times{ }_{\mathrm{G}} \mathrm{L}^{1} \times{ }_{\mathrm{G}} \mathrm{X} \rightarrow \mathrm{K}^{0}$ such that $\mathrm{r}_{\mathrm{R}} \circ \mathrm{p}_{3}=\mathrm{r}_{\mathrm{R}}^{\prime} \circ \mathrm{pr}_{1}$. Elementwise $\mathrm{r}_{\mathrm{R}}\left(\left[[x ; l] ; x_{1}\right]\right)=\mathrm{r}_{\mathrm{R}}^{\prime}([x ; l])$. For any element

$$
\left((x ; l) ; x_{1}\right) \in\left(\mathrm{X} \times \times_{\mathrm{r}_{X}, \mathrm{~L}^{0}, \mathrm{r}_{\mathrm{L}}} \mathrm{L}^{1}\right) \times_{\mathrm{s}_{\mathrm{L}} \mathrm{Opr} r_{2}, \mathrm{~L}^{0}, \mathrm{r}_{\mathrm{X}}} \mathrm{X}
$$

we can write $\mathrm{r}_{\mathrm{R}}\left(\left[[x ; l] ; x_{1}\right]\right)=\mathrm{r}_{\mathrm{R}}^{\prime}([x ; l])=\mathrm{s}_{\mathbf{X}}(x)$. Here the element

$$
\left[[x ; l] ; x_{1}\right] \in \mathrm{X} \times \times_{\mathrm{G}} \mathrm{L}^{1} \times_{\mathrm{G}} \mathrm{X}
$$

is understood as the composition $\mathrm{p}_{3} \circ\left(\mathrm{p}_{1} \circ \mathrm{pr}_{1} ; \mathrm{pr}_{2}\right) \circ\left((x ; l) ; x_{1}\right)$.

We have two arrows, $s_{R}$ and $r_{R}$. The arrow $s_{R}$ is a cover because it is induced

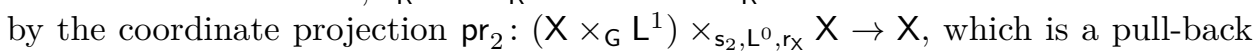
of $\mathrm{s}_{2}: \mathrm{X} \times \mathrm{G}_{\mathrm{G}} \mathrm{L}^{1} \rightarrow \mathrm{L}^{0}$, which is a cover under Assumptions 2.14 and 2.19 because $\mathrm{s}_{2} \circ \mathrm{p}_{1}=\mathrm{s}_{\mathrm{L}} \circ \mathrm{pr}_{2}$. Under these assumptions, the arrow $\mathrm{r}_{\mathrm{R}}$ is a cover, too, because $\mathrm{r}_{\mathrm{R}} \circ \mathrm{p}_{3}=\mathrm{r}_{\mathrm{R}}^{\prime} \circ \mathrm{pr}_{1}$, where $\mathrm{pr}_{1}$ is a cover, as a pull-back of the cover $\mathrm{r}_{\mathrm{X}}$, and $\mathrm{r}_{\mathrm{R}}^{\prime}$ is a

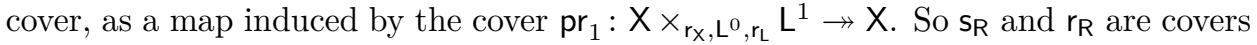
and we use them as the source and range maps of $R$, respectively.

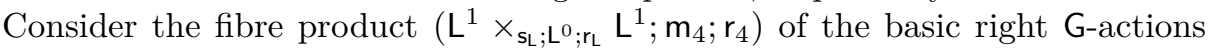
$\left(\mathrm{L}^{1}, \mathrm{~m}_{\mathrm{L}} \circ\left(\mathrm{pr}_{1} ; \mathrm{pr}_{1} \circ \mathrm{pr}_{2}\right), \mathrm{s}_{\mathrm{L}}\right)$ and $\left(\mathrm{L}^{1} ; \mathrm{m}_{\mathrm{L}} \circ\left(\mathrm{i}_{\mathrm{L}} \circ \mathrm{pr}_{1} \circ \mathrm{pr}_{2} ; \mathrm{pr}_{1}\right) ; \mathrm{r}_{\mathrm{L}}\right)$. This action is defined elementwise by

$$
\left(l_{1} ; l_{2}\right) \cdot g=\left(l_{1} \cdot g ; g^{-1} \cdot l_{2}\right)
$$

for all $g \in \mathrm{G}^{1}, l_{1}, l_{2} \in \mathrm{L}^{1}$ with $\mathrm{s}_{\mathrm{L}}\left(l_{1}\right)=\mathrm{r}_{\mathrm{G}}(g)=\mathrm{r}_{\mathrm{L}}\left(l_{2}\right)$. We know that this action is basic by Assumption 5.63 Let the cover $\mathrm{p}_{4}: \mathrm{L}^{1} \times_{\mathrm{sL}_{\mathrm{L}} \mathrm{L}^{0}} \mathrm{~L}^{1} \rightarrow \mathrm{L}^{1} \times_{\mathrm{G}} \mathrm{L}^{1}$ be the orbit space projection of this action. The multiplication map of the groupoid $\mathrm{L}$ is G-invariant because $\left(l_{1} \cdot g\right) \cdot\left(g^{-1} \cdot l_{2}\right)=l_{1} \cdot\left(g \cdot g^{-1}\right) \cdot l_{2}=l_{1} \cdot l_{2}$. Therefore, there is a unique arrow $\mathrm{m}_{\mathrm{R}}^{\prime}: \mathrm{L}^{1} \times_{\mathrm{G}} \mathrm{L}^{1} \rightarrow \mathrm{L}^{1}$ such that $\mathrm{m}_{\mathrm{R}}^{\prime} \circ \mathrm{p}_{4}=\mathrm{m}_{\mathrm{L}}$. Elementwise $\mathrm{m}_{\mathrm{R}}^{\prime}\left(\left[l_{1} ; l_{2}\right]\right)=l_{1} \cdot l_{2}$. We use this map to define the multiplication map $\mathrm{m}_{\mathrm{R}}$.

We know that the bibundle equivalence $\left(X ; m_{1 X} ; m_{2 X} ; s_{X} ; r_{X}\right) \equiv \mathbb{X}$ from $G$ to $K$ is a bibundle actor from $G$ to $K$. Let $\mathbb{X}^{-1}$ be the bibundle actor from $K$ to $G$, described in Lemma 6.22 Let $\mathbb{L}^{1}$ be the bibundle actor from $\mathrm{G}$ to itself described in Example 6.8 By construction, the object of the composition of bibundle actors

$$
\mathbb{X} \circ\left(\mathbb{L}^{1} \circ \mathbb{X}^{-1}\right)
$$

is $\mathrm{R}^{1}=\mathrm{X} \times{ }_{\mathrm{G}} \mathrm{L}^{1} \times_{\mathrm{G}} \mathrm{X}$. We know that the composition of bibundle actors is associative. Thus $\mathbb{X} \circ\left(\mathbb{L}^{1} \circ \mathbb{X}^{-1}\right) \cong\left(\mathbb{X} \circ \mathbb{L}^{1}\right) \circ \mathbb{X}^{-1}$. So an element $\left[[x ; l] ; x_{1}\right]$ of $\mathrm{R}^{1}=\mathrm{X} \times{ }_{\mathrm{G}} \mathrm{L}^{1} \times{ }_{\mathrm{G}} \mathrm{X}$ can be understood as $\mathrm{p}_{3}^{\prime} \circ\left(\mathrm{pr}_{1} ; \mathrm{p}_{1}^{\prime} \circ \mathrm{pr}_{2}\right) \circ\left(x ;\left(l ; x_{1}\right)\right)$, we just write $\left[x ; l ; x_{1}\right]$.

The object of the composition $\mathbb{L}^{1} \circ \mathbb{L}^{1}$ is $\mathrm{L}^{1} \times{ }_{\mathrm{G}} \mathrm{L}^{1}$, by construction. We have the left and right $\mathrm{G}$-actions on $\mathrm{L}^{1} \times{ }_{\mathrm{G}} \mathrm{L}^{1}$ defined elementwise by $g \cdot\left[l_{1} ; l_{2}\right]=\left[g \cdot l_{1} ; l_{2}\right]$ and $\left[l_{1} ; l_{2}\right] \cdot g=\left[l_{1} ; l_{2} \cdot g\right]$, which define the bibundle actor $\mathbb{L}^{1} \circ \mathbb{L}^{1}$ from $\mathrm{G}$ to itself. In the sense of these actions the arrow $m_{R}^{\prime}: L^{1} \times{ }_{G} L^{1} \rightarrow L^{1}$ is $G, G$-map because

$$
\begin{aligned}
\mathrm{m}_{\mathrm{R}}^{\prime}\left(g \cdot\left[l_{1} ; l_{2}\right]\right) & =\mathrm{m}_{\mathrm{R}}^{\prime}\left(\left[g \cdot l_{1} ; l_{2}\right]\right) \\
& =\left(g \cdot l_{1}\right) \cdot l_{2} \\
& =g \cdot\left(l_{1} \cdot l_{2}\right) \\
& =g \cdot \mathrm{m}_{\mathrm{R}}^{\prime}\left(\left[l_{1} ; l_{2}\right]\right)
\end{aligned}
$$

and,

$$
\begin{aligned}
\mathrm{m}_{\mathrm{R}}^{\prime}\left(\left[l_{1} ; l_{2}\right] \cdot g\right) & =\mathrm{m}_{\mathrm{R}}^{\prime}\left(\left[l_{1} ; l_{2} \cdot g\right]\right) \\
& =l_{1} \cdot\left(l_{2} \cdot g\right) \\
& =\left(l_{1} \cdot l_{2}\right) \cdot g \\
& =\mathrm{m}_{\mathrm{R}}^{\prime}\left(\left[l_{1} ; l_{2}\right]\right) \cdot g .
\end{aligned}
$$

Therefore, we have an induced G-map $\mathrm{m}_{\mathrm{R}}^{\prime \prime}: \mathrm{X} \times{ }_{\mathrm{G}} \mathrm{L}^{1} \times{ }_{\mathrm{G}} \mathrm{L}^{1} \times_{\mathrm{G}} \mathrm{X} \rightarrow \mathrm{X} \times \times_{\mathrm{G}} \mathrm{L}^{1} \times{ }_{\mathrm{G}} \mathrm{X}$ by Remark 6.10 It is defined elementwise by $\mathrm{m}_{\mathrm{R}}^{\prime \prime}\left(\left[x_{1} ; l_{1} ; l_{2} ; x_{2}\right]\right)=\left[x_{1} ; l_{1} \cdot l_{2} ; x_{2}\right]$. Since 
the composition of bibundle actors is associative, which is explained in Remark 6.18 we have the following natural isomorphisms:

$$
\begin{aligned}
\left(\mathbb{X} \circ \mathbb{L}^{1} \circ \mathbb{X}^{-1}\right) \circ\left(\mathbb{X} \circ \mathbb{L}^{1} \circ \mathbb{X}^{-1}\right) & \cong \mathbb{X} \circ \mathbb{L}^{1} \circ\left(\mathbb{X}^{-1} \circ \mathbb{X}\right) \circ \mathbb{L}^{1} \circ \mathbb{X}^{-1} \\
& \cong \mathbb{X} \circ \mathbb{L}^{1} \circ \mathbb{G}^{1} \circ \mathbb{L}^{1} \circ \mathbb{X}^{-1} \\
& \cong \mathbb{X} \circ \mathbb{L}^{1} \circ \mathbb{L}^{1} \circ \mathbb{X}^{-1}
\end{aligned}
$$

where $\mathbb{G}^{1}$ is the unit bibundle actor from $G$ to itself. Hence there is the following arrow

$$
\mathrm{m}_{\mathrm{R}}^{\prime \prime \prime}:\left(\mathrm{X} \times \times_{\mathrm{G}} \mathrm{L}^{1} \times_{\mathrm{G}} \mathrm{X}\right) \times_{\mathrm{K}}\left(\mathrm{X} \times_{\mathrm{G}} \mathrm{L}^{1} \times_{\mathrm{G}} \mathrm{X}\right) \rightarrow \mathrm{X} \times_{\mathrm{G}} \mathrm{L}^{1} \times_{\mathrm{G}} \mathrm{X},
$$

defined elementwise by

$$
\mathrm{m}_{\mathrm{R}}^{\prime \prime \prime}\left(\left[\left[x ; l ; x_{1}\right] ;\left[x^{\prime} ; l^{\prime} ; x_{1}^{\prime}\right]\right]\right)=\left[x ; l \cdot g \cdot l^{\prime} ; x_{1}^{\prime}\right]
$$

for all $x, x_{1}, x^{\prime}, x_{1}^{\prime} \in \mathrm{X}, l, l^{\prime} \in \mathrm{L}^{1}$ with $\mathrm{r}_{\mathbf{X}}(x)=\mathrm{r}_{\mathrm{L}}(l), \mathrm{r}_{\mathbf{X}}\left(x_{1}\right)=\mathrm{s}_{\mathrm{L}}(l), \mathrm{r}_{\mathbf{X}}\left(x^{\prime}\right)=\mathrm{r}_{\mathrm{L}}\left(l^{\prime}\right)$, $\mathrm{r}_{\mathrm{X}}\left(x_{1}^{\prime}\right)=\mathrm{s}_{\mathrm{L}}\left(l^{\prime}\right)$ and $\mathrm{s}_{\mathrm{X}}\left(x_{1}\right)=\mathrm{s}_{\mathrm{X}}\left(x^{\prime}\right)$, where $g \in \mathrm{G}^{1}$ is the element which is given by the following composition $\mathrm{pr}_{1} \circ\left(\mathrm{pr}_{1} ; \mathrm{m}_{1 \mathrm{X}}\right)^{-1} \circ\left(x_{2} ; x_{1}^{\prime}\right)$. Now, we can define the multiplication map of the groupoid $R$. Let $m_{R}=m_{R}^{\prime \prime \prime} \circ p^{\prime \prime \prime}$, where $p^{\prime \prime \prime}$ is an orbit space projection of the right $\mathrm{K}$-action on $\left(X \times{ }_{G} L^{1} \times_{G} X\right) \times_{S_{R}, K^{0}, r_{R}}\left(X \times{ }_{G} L^{1} \times_{G} X\right)$ defined elementwise by

$$
\left(\left[x ; l ; x_{1}\right] ;\left[x^{\prime} ; l^{\prime} ; x_{1}^{\prime}\right]\right) \cdot k=\left(\left[x ; l ; x_{1} \cdot k\right] ;\left[x^{\prime} \cdot k ; l^{\prime} ; x_{1}^{\prime}\right]\right)
$$

for all $x, x_{1}, x^{\prime}, x_{1}^{\prime} \in \mathrm{X}, l, l^{\prime} \in \mathrm{L}^{1}, k \in \mathrm{K}^{1}$ with $\mathrm{sX}_{\mathrm{X}}\left(x_{1}\right)=\mathrm{s}_{\mathrm{X}}\left(x^{\prime}\right)=\mathrm{r}_{\mathrm{K}}(k), \mathrm{rX}_{\mathrm{X}}(x)=\mathrm{r}_{\mathrm{L}}(l)$, $\mathrm{r}_{\mathbf{X}}\left(x_{1}\right)=\mathrm{s}_{\mathrm{L}}(l), \mathrm{r}_{\mathbf{X}}\left(x^{\prime}\right)=\mathrm{r}_{\mathrm{L}}\left(l^{\prime}\right)$ and $\mathrm{r}_{\mathbf{X}}\left(x_{1}^{\prime}\right)=\mathrm{s}_{\mathrm{L}}\left(l^{\prime}\right)$. So the multiplication map of the groupoid $\mathrm{R}$ is defined elementwise by

$$
\left[x ; l ; x_{1}\right] \cdot\left[x^{\prime} ; l^{\prime} ; x_{1}^{\prime}\right]=\left[x ; l \cdot g \cdot l^{\prime} ; x_{1}^{\prime}\right]
$$

for all $x, x_{1}, x^{\prime}, x_{1}^{\prime} \in \mathrm{X}, l, l^{\prime} \in \mathrm{L}^{1}$ with $\mathrm{r}_{\mathbf{X}}(x)=\mathrm{r}_{\mathrm{L}}(l), \mathrm{r}_{\mathbf{X}}\left(x_{1}\right)=\mathrm{s}_{\mathrm{L}}(l), \mathrm{r}_{\mathrm{X}}\left(x^{\prime}\right)=\mathrm{r}_{\mathrm{L}}\left(l^{\prime}\right)$, $\mathrm{r}_{\mathrm{X}}\left(x_{1}^{\prime}\right)=\mathrm{s}_{\mathrm{L}}\left(l^{\prime}\right)$ and $\mathrm{s}_{\mathrm{X}}\left(x_{1}\right)=\mathrm{s}_{\mathrm{X}}\left(x^{\prime}\right)$, where $g \in \mathrm{G}^{1}$ is the element considered above.

Consider the following composition

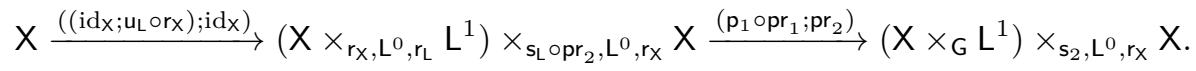

It is a G-map in the sense of the right G-actions $\left(X ; m_{1 X} \circ\left(i_{G} \circ p r_{2} ; p_{1}\right) ; r_{X}\right)$ and $\left(\left(X \times_{G} L^{1}\right) \times_{s_{2}, L^{0}, r_{X}} X ; m_{3} ; r_{3}\right)$ described above because it clearly commutes with anchor maps and

$$
\begin{aligned}
\left(\left[x \cdot g ; 1_{\mathrm{r}_{\mathrm{X}}(x \cdot g)}\right] ; x \cdot g\right) & =\left(\left[g^{-1} \cdot x ; 1_{\mathrm{r}_{\mathbf{X}}\left(g^{-1} \cdot x\right)}\right] ; g^{-1} \cdot x\right) \\
& =\left(\left[g \cdot g^{-1} \cdot x ; g \cdot 1_{\mathrm{r}_{\mathbf{X}}\left(g^{-1} \cdot x\right)}\right] ; g^{-1} \cdot x\right) \\
= & \left([x ; g] ; g^{-1} \cdot x\right) \\
= & \left(\left[x ; 1_{\mathrm{r}_{\mathrm{G}}(g)}\right] \cdot g ; g^{-1} \cdot x\right) \\
= & \left(\left[x ; 1_{\mathrm{r}_{\mathbf{X}}(x)}\right] \cdot g ; x \cdot g\right) \\
= & \left(\left[x ; 1_{\mathrm{rX}_{\mathrm{X}}(x)}\right] ; x\right) \cdot g .
\end{aligned}
$$

Therefore, there is a unique arrow $\mathrm{u}_{\mathrm{R}}: \mathrm{K}^{0} \rightarrow \mathrm{X} \times{ }_{\mathrm{G}} \mathrm{L}^{1} \times_{\mathrm{G}} \mathrm{X}$ such that

$$
\mathrm{u}_{\mathrm{R}} \circ \mathrm{s}_{\mathrm{X}}=\mathrm{p}_{3} \circ\left(\mathrm{p}_{1} \circ \mathrm{pr}_{1} ; \mathrm{pr}_{2}\right) \circ\left(\left(\mathrm{id}_{\mathrm{X}} ; \mathrm{u}_{\mathrm{L}} \circ \mathrm{r}_{\mathrm{X}}\right) ; \mathrm{id}_{\mathrm{X}}\right), \quad \mathrm{u}_{\mathrm{R}}\left(k_{0}\right)=\left[x ; 1_{\mathrm{rX}(x)} ; x\right]
$$

for all $k_{0} \in \mathrm{K}^{0}, x \in \mathrm{X}$ with $\mathrm{sx}(x)=k_{0}$.

Consider the following isomorphism

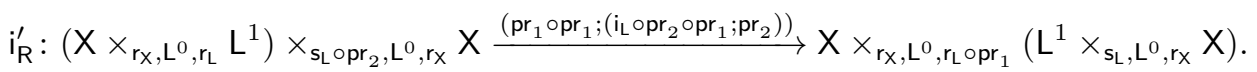

Elementwise $\mathrm{i}_{\mathrm{R}}^{\prime}\left((x ; l) ; x_{1}\right)=\left(x_{1} ;\left(l^{-1} ; x\right)\right)$. This isomorphism is a $\mathrm{G}$-map in the sense of the G-actions defined elementwise by $\left((x ; l) ; x_{1}\right) \cdot g=\left(\left(g^{-1} \cdot x ; g^{-1} \cdot l\right) ; x_{1}\right)$ and 
$\left(x ;\left(l ; x_{1}\right)\right) \cdot g=\left(x ;\left(l \cdot g ; g^{-1} \cdot x_{1}\right)\right.$, respectively, because it clearly commutes with anchor maps and

$$
\begin{aligned}
\mathrm{i}_{\mathrm{R}}^{\prime}\left((x ; l) ; x_{1}\right) \cdot g & =\left(x_{1} ;\left(l^{-1} ; x\right)\right) \cdot g \\
& =\left(x_{1} ;\left(l^{-1} \cdot g ; g^{-1} \cdot x\right)\right) \\
& =\left(x_{1} ;\left(\left(g^{-1} \cdot l\right)^{-1} ; g^{-1} \cdot x\right)\right) \\
& =\mathrm{i}_{\mathrm{R}}^{\prime}\left(\left(g^{-1} \cdot x ; g^{-1} \cdot l\right) ; x_{1}\right) \\
& =\mathrm{i}_{\mathrm{R}}^{\prime}\left(\left((x ; l) ; x_{1}\right) \cdot g\right)
\end{aligned}
$$

for all $x, x_{1} \in \mathrm{X}, g \in \mathrm{G}^{1}, l \in \mathrm{L}^{1}$ with $\mathrm{r}_{\mathrm{X}}(x)=\mathrm{r}_{\mathrm{L}}(l)=\mathrm{r}_{\mathrm{G}}(g)$ and $\mathrm{r}_{\mathrm{X}}\left(x_{1}\right)=\mathrm{s}_{\mathrm{L}}(l)$. Hence it induces an isomorphism

$$
\mathrm{i}_{\mathrm{R}}^{\prime \prime}:\left(\mathrm{X} \times_{\mathrm{G}} \mathrm{L}^{1}\right) \times_{\mathrm{s}_{2}, \mathrm{~L}^{0}, \mathrm{rX}_{\mathrm{X}}} \mathrm{X} \rightarrow \mathrm{X} \times_{\mathrm{rx}_{\mathrm{L}} \mathrm{L}^{0}, \mathrm{r}_{2}}\left(\mathrm{~L}^{1} \times_{\mathrm{G}} \mathrm{X}\right)
$$

such that $\mathrm{i}_{\mathrm{R}}^{\prime \prime}\left([x ; l] ; x_{1}\right)=\left(x_{1} ;\left[l^{-1} ; x\right]\right)$. We also have the $\mathrm{G}$-actions defined elementwise by $\left([x ; l] ; x_{1}\right) \cdot g=\left([x ; l \cdot g] ; g^{-1} \cdot x_{1}\right)$ and $\left(x ;\left[l ; x_{1}\right]\right) \cdot g=\left(g^{-1} \cdot x ;\left[g^{-1} \cdot l ; x_{1}\right]\right)$. The isomorphism $\mathrm{i}_{\mathrm{R}}^{\prime \prime}$ is a $\mathrm{G}$-map in the sense of these actions because it clearly commutes with anchor maps and

$$
\begin{aligned}
\mathrm{i}_{\mathrm{R}}^{\prime \prime}\left([x ; l] ; x_{1}\right) \cdot g & =\left(x_{1} ;\left[l^{-1} ; x\right]\right) \cdot g \\
& =\left(g^{-1} \cdot x_{1} ;\left[g^{-1} \cdot l^{-1} ; x\right]\right) \\
& =\left(g^{-1} \cdot x_{1} ;\left[(l \cdot g)^{-1} ; x\right]\right) \\
& =\mathrm{i}_{\mathrm{R}}^{\prime \prime}\left([x ; l \cdot g] ; g^{-1} \cdot x_{1}\right) \\
& =\mathrm{i}_{\mathrm{R}}^{\prime \prime}\left(\left([x ; l] ; x_{1}\right) \cdot g\right)
\end{aligned}
$$

for all $x, x_{1} \in \mathrm{X}, g \in \mathrm{G}^{1}, l \in \mathrm{L}^{1}$ with $\mathrm{r}_{\mathrm{X}}(x)=\mathrm{r}_{\mathrm{L}}(l)$ and $\mathrm{r}_{\mathrm{X}}\left(x_{1}\right)=\mathrm{s}_{\mathrm{L}}(l)=\mathrm{r}_{\mathrm{G}}(g)$. Therefore, it induces the following isomorphism

$$
\mathrm{i}_{\mathrm{R}}: \mathrm{X} \times \times_{\mathrm{G}} \mathrm{L}^{1} \times{ }_{\mathrm{G}} \mathrm{X} \rightarrow \mathrm{X} \times \times_{\mathrm{G}} \mathrm{L}^{1} \times{ }_{\mathrm{G}} \mathrm{X},
$$

such that $\mathrm{i}_{\mathrm{R}}\left(\left[x ; l ; x_{1}\right]\right)=\left[x_{1} ; l^{-1} ; x\right]$ for all $x, x_{1} \in \mathrm{X}, l \in \mathrm{L}^{1}$ with $\mathrm{r}_{\mathrm{X}}(x)=\mathrm{r}_{\mathrm{L}}(l)$ and $r_{\mathbf{X}}\left(x_{1}\right)=\mathrm{s}_{\mathrm{L}}(l)$. We are going to show that the data $\mathrm{R}=\left(\mathrm{R}^{0}, \mathrm{R}^{1}, \mathrm{r}_{\mathrm{R}}, \mathrm{s}_{\mathrm{R}}, \mathrm{m}_{\mathrm{R}}, \mathrm{u}_{\mathrm{R}}, \mathrm{i}_{\mathrm{R}}\right)$ defined above is a groupoid in the category $\left(\mathcal{C}, \mathcal{F}_{p}\right)$ with partial covers.

We have shown above that $r_{R}$ and $s_{R}$ are covers, which is the property (1) Now let us check the property $(2)$. Let $\left[x ; l ; x_{1}\right]$ and $\left[x^{\prime} ; l^{\prime} ; x_{1}^{\prime}\right]$ be composable pairs in $\mathrm{R}^{1}$. We have

$$
\begin{aligned}
\mathrm{r}_{\mathrm{R}}\left(\left[x ; l ; x_{1}\right] \cdot\left[x^{\prime} ; l^{\prime} ; x_{1}^{\prime}\right]\right) & =\mathrm{r}_{\mathrm{R}}\left(\left[x ; l \cdot g \cdot l^{\prime} ; x_{1}^{\prime}\right]\right) \\
& =\mathrm{sx}_{\mathrm{X}}(x) \\
& =\mathrm{r}_{\mathrm{R}}\left(\left[x ; l ; x_{1}\right]\right)
\end{aligned}
$$

and

$$
\begin{aligned}
\mathrm{s}_{\mathrm{R}}\left(\left[x ; l ; x_{1}\right] \cdot\left[x^{\prime} ; l^{\prime} ; x_{1}^{\prime}\right]\right) & =\mathrm{s}_{\mathrm{R}}\left(\left[x ; l \cdot g \cdot l^{\prime} ; x_{1}^{\prime}\right]\right) \\
& =\mathrm{s}_{\mathrm{X}}\left(x_{1}^{\prime}\right) \\
& =\mathrm{s}_{\mathrm{R}}\left(\left[x^{\prime} ; l^{\prime} ; x_{1}^{\prime}\right]\right)
\end{aligned}
$$

for all $x, x_{1}, x^{\prime}, x_{1}^{\prime} \in \mathrm{X}, l, l^{\prime} \in \mathrm{L}^{1}$ with $\mathrm{s}_{\mathrm{X}}\left(x_{1}\right)=\mathrm{s}_{\mathbf{X}}\left(x^{\prime}\right), \mathrm{r}_{\mathbf{X}}(x)=\mathrm{r}_{\mathrm{L}}(l), \mathrm{r}_{\mathbf{X}}\left(x_{1}\right)=\mathrm{s}_{\mathrm{L}}(l)$, $\mathrm{r}_{\mathrm{X}}\left(x^{\prime}\right)=\mathrm{r}_{\mathrm{L}}\left(l^{\prime}\right)$ and $\mathrm{r}_{\mathrm{X}}\left(x_{1}^{\prime}\right)=\mathrm{s}_{\mathrm{L}}\left(l^{\prime}\right)$. So we have the property $(2)$.

Consider a composable triple of arrows in $\mathrm{R}^{1}$. We have

$$
\begin{aligned}
\left(\left[x ; l ; x_{1}\right] \cdot\left[x^{\prime} ; l^{\prime} ; x_{1}^{\prime}\right]\right) \cdot\left[x^{\prime \prime} ; l^{\prime \prime} ; x_{1}^{\prime \prime}\right] & =\left[x ; l \cdot g \cdot l^{\prime} ; x_{1}^{\prime}\right] \cdot\left[x^{\prime \prime} ; l^{\prime \prime} ; x_{1}^{\prime \prime}\right] \\
& =\left[x ;\left(l \cdot g \cdot l^{\prime}\right) \cdot g_{1} \cdot l^{\prime \prime} ; x_{1}^{\prime \prime}\right],
\end{aligned}
$$

and

$$
\begin{aligned}
{\left[x ; l ; x_{1}\right] \cdot\left(\left[x^{\prime} ; l^{\prime} ; x_{1}^{\prime}\right] \cdot\left[x^{\prime \prime} ; l^{\prime \prime} ; x_{1}^{\prime \prime}\right]\right) } & =\left[x ; l ; x_{1}\right] \cdot\left[x^{\prime} ; l^{\prime} \cdot g_{1}^{\prime} \cdot l^{\prime \prime} ; x_{1}^{\prime \prime}\right] \\
& =\left[x ; l \cdot g^{\prime} \cdot\left(l^{\prime} \cdot g_{1}^{\prime} \cdot l^{\prime \prime}\right) ; x_{1}^{\prime \prime}\right]
\end{aligned}
$$


By definition of the multiplication in $\mathrm{R}^{1}$ we know that the elements $g \in \mathrm{G}^{1}$ and $g^{\prime} \in \mathrm{G}^{1}$ are given by the composition $\mathrm{pr}_{1} \circ\left(\mathrm{pr}_{1} ; \mathrm{m}_{1 \mathrm{X}}\right)^{-1} \circ\left(x_{1} ; x^{\prime}\right)$. So $g=g^{\prime}$. For the same reason $g_{1}=g_{1}^{\prime}$. Hence

$$
\left(\left[x ; l ; x_{1}\right] \cdot\left[x^{\prime} ; l^{\prime} ; x_{1}^{\prime}\right]\right) \cdot\left[x^{\prime \prime} ; l^{\prime \prime} ; x_{1}^{\prime \prime}\right]=\left[x ; l ; x_{1}\right] \cdot\left(\left[x^{\prime} ; l^{\prime} ; x_{1}^{\prime}\right] \cdot\left[x^{\prime \prime} ; l^{\prime \prime} ; x_{1}^{\prime \prime}\right]\right) .
$$

Therefore, the multiplication map $m_{R}$ is associative. This is (3)

We are going to check all properties in Proposition 3.8 and deduce that $\mathrm{R}$ is a groupoid by Proposition 3.11 Consider any element $k_{0}$ in $\mathrm{R}^{0}$ and any element $x$ in $\mathrm{X}$ such that $\mathrm{s}_{\mathbf{X}}(x)=k_{0}$. We have $\mathrm{r}_{\mathrm{R}}\left(\mathrm{u}_{\mathrm{R}}\left(k_{0}\right)\right)=\mathrm{r}_{\mathrm{R}}\left(\left[x ; 1_{\mathrm{r}_{X}(x)} ; x\right]\right)=\mathrm{s}_{\mathbf{X}}(x)=k_{0}$, and $\mathrm{s}_{\mathrm{R}}\left(\mathrm{u}_{\mathrm{R}}\left(k_{0}\right)\right)=\mathrm{s}_{\mathrm{R}}\left(\left[x ; 1_{\mathrm{r}_{\mathrm{X}}(x)} ; x\right]\right)=\mathrm{s}_{\mathrm{X}}(x)=k_{0}$. Thus the property $(1)$ holds. Also, we have

$$
\begin{aligned}
\mathrm{u}_{\mathrm{R}}\left(\mathrm{r}_{\mathrm{R}}\left(\left[x ; l ; x_{1}\right]\right)\right) \cdot\left[x ; l ; x_{1}\right] & =\mathrm{u}_{\mathrm{R}}\left(\mathrm{s}_{\mathbf{X}}(x)\right) \cdot\left[x ; l ; x_{1}\right] \\
& =\left[x ; 1_{\mathrm{r}_{\mathbf{x}}(x)} ; x\right] \cdot\left[x ; l ; x_{1}\right] \\
& =\left[x ; 1_{\mathrm{r}_{\mathbf{x}}(x)} \cdot 1_{\mathrm{r}_{\mathbf{X}}(x)} \cdot l ; x_{1}\right] \\
& =\left[x ; l ; x_{1}\right] .
\end{aligned}
$$

We have used here that the composition $\mathrm{pr}_{1} \circ\left(\mathrm{pr}_{1} ; \mathrm{m}_{1 \mathrm{X}}\right)^{-1} \circ(x ; x)$ clearly gives the element $1_{\mathrm{r}_{\mathrm{X}}(x)}$. Analogously, we have

$$
\begin{aligned}
{\left[x ; l ; x_{1}\right] \cdot \mathrm{u}_{\mathrm{R}}\left(\mathrm{s}_{\mathrm{R}}\left(\left[x ; l ; x_{1}\right]\right)\right) } & =\left[x ; l ; x_{1}\right] \cdot \mathrm{u}_{\mathrm{R}}\left(\mathrm{s}_{\mathrm{R}}\left(x_{1}\right)\right) \\
& =\left[x ; l ; x_{1}\right] \cdot\left[x_{1} ; 1_{\mathrm{r}_{x}\left(x_{1}\right)} ; x_{1}\right] \\
& =\left[x ; l \cdot 1_{\mathrm{r}_{x}\left(x_{1}\right)} \cdot 1_{\mathrm{r}_{\mathrm{x}}\left(x_{1}\right)} ; x_{1}\right. \\
& =\left[x ; l ; x_{1}\right] .
\end{aligned}
$$

Therefore, the property (2) holds. The property (3) holds obviously:

$$
\begin{aligned}
\mathrm{r}_{\mathrm{R}}\left(\mathrm{i}_{\mathrm{R}}\left(\left[x ; l ; x_{1}\right]\right)\right) & =\mathrm{r}_{\mathrm{R}}\left(\left[x_{1} ; l^{-1} ; x\right]\right) \\
& =\mathrm{s}_{\mathrm{X}}\left(x_{1}\right) \\
& =\mathrm{s}_{\mathrm{R}}\left(\left[x ; l ; x_{1}\right]\right),
\end{aligned}
$$

and

$$
\begin{aligned}
\mathrm{s}_{\mathrm{R}}\left(\mathrm{i}_{\mathrm{R}}\left(\left[x ; l ; x_{1}\right]\right)\right) & =\mathrm{s}_{\mathrm{R}}\left(\left[x_{1} ; l^{-1} ; x\right]\right) \\
& =\mathrm{s}_{\mathrm{X}}(x) \\
& =\mathrm{r}_{\mathrm{R}}\left(\left[x ; l ; x_{1}\right]\right) .
\end{aligned}
$$

We need to check one more property. That is (4). We have

$$
\begin{aligned}
\mathrm{i}_{\mathrm{R}}\left(\left[x ; l ; x_{1}\right]\right) \cdot\left[x ; l ; x_{1}\right] & =\left[x_{1} ; l^{-1} ; x\right] \cdot\left[x ; l ; x_{1}\right] \\
& =\left[x_{1} ; l^{-1} \cdot 1_{\mathrm{rx}(x)} \cdot l ; x_{1}\right] \\
& =\left[x_{1} ; 1_{\mathrm{rx}\left(x_{1}\right)} ; x_{1}\right] \\
& =\mathrm{u}_{\mathrm{R}}\left(\mathrm{s}_{\mathbf{X}}\left(x_{1}\right)\right) \\
& =\mathrm{u}_{\mathrm{R}}\left(\mathrm{s}_{\mathrm{R}}\left(\left[x ; l ; x_{1}\right]\right)\right),
\end{aligned}
$$

and

$$
\begin{aligned}
{\left[x ; l ; x_{1}\right] \cdot \mathrm{i}_{\mathrm{R}}\left(\left[x ; l ; x_{1}\right]\right) } & =\left[x ; l ; x_{1}\right] \cdot\left[x_{1} ; l^{-1} ; x\right] \\
& =\left[x ; l \cdot 1_{\mathrm{r}_{\mathbf{x}}\left(x_{1}\right)} \cdot l^{-1} ; x\right] \\
& =\left[x ; 1_{\mathrm{r}_{X}(x)} ; x\right] \\
& =\mathrm{u}_{\mathrm{R}}\left(\mathrm{s}_{X}(x)\right) \\
& =\mathrm{u}_{\mathrm{R}}\left(\mathrm{r}_{\mathrm{R}}\left(\left[x ; l ; x_{1}\right]\right)\right) .
\end{aligned}
$$

So we have all required properties in Proposition 3.11 Therefore, we can deduce that $\mathrm{R}=\left(\mathrm{R}^{0}, \mathrm{R}^{1}, \mathrm{r}_{\mathrm{R}}, \mathrm{s}_{\mathrm{R}}, \mathrm{m}_{\mathrm{R}}, \mathrm{u}_{\mathrm{R}}, \mathrm{i}_{\mathrm{R}}\right)$ is a groupoid by Proposition 3.11. 
The next step is to construct the groupoid fibration from $\mathrm{R}$ to $\mathrm{H}$. Consider the following composition

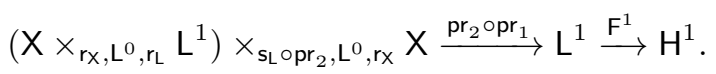

It is G-invariant in the sense of the G-action on $\left(X \times_{\left.r_{X}, L^{0}, r_{\mathrm{L}} L^{1}\right)} \times_{\mathrm{s}_{\mathrm{L}} \circ \mathrm{pr}_{2}, \mathrm{~L}^{0}, \mathrm{rX}_{\mathrm{X}}} \mathrm{X}\right.$ defined elementwise by $\left((x ; l) ; x_{1}\right) \cdot g=\left(\left(g^{-1} \cdot x ; g^{-1} \cdot l\right) ; x_{1}\right)$ because

$$
\begin{aligned}
\mathrm{F}^{1}\left(\operatorname{pr}_{2}\left(\operatorname{pr}_{1}\left(\left((x ; l) ; x_{1}\right) \cdot g\right)\right)\right) & =\mathrm{F}^{1}\left(\operatorname{pr}_{2}\left(\operatorname{pr}_{1}\left(\left(g^{-1} \cdot x ; g^{-1} \cdot l\right) ; x_{1}\right)\right)\right) \\
& =\mathrm{F}^{1}\left(g^{-1} \cdot l\right) \\
& =\mathrm{F}^{1}(l) \\
& =\mathrm{F}^{1}\left(\operatorname{pr}_{2}\left(\operatorname{pr}_{1}\left((x ; l) ; x_{1}\right)\right)\right)
\end{aligned}
$$

Therefore, there is a unique arrow $\tilde{E}^{1}:\left(X \times{ }_{G} L^{1}\right) \times_{s_{2}, L^{0}, r_{X}} X \rightarrow H^{1}$ such that $\tilde{E}^{1} \circ$ $\left(\mathrm{p}_{1} \circ \mathrm{pr}_{1} ; \mathrm{pr}_{2}\right)=\mathrm{F}^{1} \circ \mathrm{pr}_{2} \circ \mathrm{pr}_{1}$. Elementwise $\tilde{\mathrm{E}}^{1}\left([x ; l] ; x_{1}\right)=\mathrm{F}^{1}(l)$. The arrow $\tilde{\mathrm{E}}^{1}$ is $\mathrm{G}$-invariant in the sense of $\mathrm{G}$-action on $\left(\mathrm{X} \times{ }_{\mathrm{G}} \mathrm{L}^{1}\right) \times_{\mathrm{s}_{2}, \mathrm{~L}^{0}, \mathrm{r}_{\mathbf{X}}} \mathrm{X}$ defined elementwise by $\left([x ; l] ; x_{1}\right) \cdot g=\left([x ; l \cdot g] ; g^{-1} \cdot x_{1}\right)$ because

$$
\begin{aligned}
\tilde{\mathrm{E}}^{1}\left(\left([x ; l] ; x_{1}\right) \cdot g\right) & =\tilde{\mathrm{E}}^{1}\left([x ; l \cdot g] ; g^{-1} \cdot x_{1}\right) \\
& =\mathrm{F}^{1}(l \cdot g) \\
& =\mathrm{F}^{1}(l) \\
& =\tilde{\mathrm{E}}^{1}\left([x ; l] ; x_{1}\right) .
\end{aligned}
$$

Therefore, there is a unique arrow $\mathrm{E}^{1}: \mathrm{X} \times{ }_{\mathrm{G}} \mathrm{L}^{1} \times{ }_{\mathrm{G}} \mathrm{X} \rightarrow \mathrm{H}^{1}$ such that $\mathrm{E}^{1} \circ \mathrm{p}_{3}=\tilde{\mathrm{E}}^{1}$. Elementwise $\mathrm{E}^{1}\left(\left[x ; l ; x_{1}\right]\right)=\tilde{\mathrm{E}}^{1}\left([x ; l] ; x_{1}\right)$. For any element

$$
\left((x ; l) ; x_{1}\right) \in\left(\mathrm{X} \times \times_{\mathrm{rX}_{\mathrm{X}}, \mathrm{L}^{0}, \mathrm{r}_{\mathrm{L}}} \mathrm{L}^{1}\right) \times_{\mathrm{sL}_{\mathrm{O} \mathrm{opr}}, \mathrm{L}^{0}, \mathrm{rX}_{\mathrm{X}}} \mathrm{X}
$$

we can write $\mathrm{E}^{1}\left(\left[x ; l ; x_{1}\right]\right)=\tilde{\mathrm{E}}^{1}\left([x ; l] ; x_{1}\right)=\mathrm{F}^{1}(l)$. Here the element

$$
\left[x ; l ; x_{1}\right] \in \mathrm{X} \times_{\mathrm{G}} \mathrm{L}^{1} \times_{\mathrm{G}} \mathrm{X}
$$

is understood as the following composition $\mathrm{p}_{3} \circ\left(\mathrm{p}_{1} \circ \mathrm{pr}_{1} ; \mathrm{pr}_{2}\right) \circ\left((x ; l) ; x_{1}\right)$. So we have the arrow $E^{1}: \mathrm{R}^{1} \rightarrow \mathrm{H}^{1}$. Let us show that the composition $\mathrm{E}^{0} \circ \mathrm{r}_{\mathrm{X}}: \mathrm{X} \rightarrow \mathrm{H}^{0}$ is G-invariant in the sense of the G-action $\left(X ; m_{1 X} ; r_{X}\right)$ :

$$
\begin{aligned}
\mathrm{F}^{0}\left(\mathrm{r}_{\mathbf{X}}(g \cdot x)\right) & =\mathrm{F}^{0}\left(\mathrm{r}_{\mathrm{G}}(g)\right) \\
& =\mathrm{r}_{\mathrm{H}}\left(\mathrm{F}^{1}(g)\right) \\
& =\mathrm{r}_{\mathrm{H}}\left(\mathrm{F}^{1}\left(1_{\mathrm{s}_{\mathrm{G}}(g)}\right)\right) \\
& =\mathrm{r}_{\mathrm{H}}\left(\mathrm{F}^{1}\left(1_{\mathrm{r}_{X}(x)}\right)\right) \\
& =\mathrm{F}^{0}\left(\mathrm{r}_{\mathbf{X}}(x)\right) .
\end{aligned}
$$

Therefore, there is a unique arrow $\mathrm{E}^{0}: \mathrm{K}^{0} \rightarrow \mathrm{H}^{0}$ such that $\mathrm{E}^{0} \circ \mathrm{sx}_{\mathrm{X}}=\mathrm{F}^{0} \circ \mathrm{r}_{\mathrm{X}}$. Elementwise $\mathrm{E}^{0}\left(k_{0}\right)=\mathrm{F}^{0}\left(r_{\mathbf{X}}(x)\right)$ for all $k_{0} \in \mathrm{K}^{0}, x \in \mathrm{X}$ with $\mathrm{sx}_{\mathbf{X}}(x)=k_{0}$. We are going to show that the pair $E=\left(E^{1} ; E^{0}\right)$ defines a groupoid fibration from $\mathrm{R}$ to $\mathrm{H}$. They intertwine the source maps because

$$
\begin{aligned}
\mathrm{s}_{\mathrm{H}}\left(\mathrm{E}^{1}\left(\left[x ; l ; x_{1}\right]\right)\right) & =\mathrm{s}_{\mathrm{H}}\left(\mathrm{F}^{1}(l)\right) \\
& =\mathrm{F}^{0}\left(\mathrm{~s}_{\mathrm{L}}(l)\right) \\
& =\mathrm{F}^{0}\left(\mathrm{r}_{\mathbf{X}}\left(x_{1}\right)\right) \\
& =\mathrm{E}^{0}\left(\mathrm{~s}_{X}\left(x_{1}\right)\right) \\
& =\mathrm{E}^{0}\left(\mathrm{~s}_{\mathrm{R}}\left(\left[x ; l ; x_{1}\right]\right)\right),
\end{aligned}
$$


and analogously, they intertwine the range maps because

$$
\begin{aligned}
\mathrm{r}_{\mathrm{H}}\left(\mathrm{E}^{1}\left(\left[x ; l ; x_{1}\right]\right)\right) & =\mathrm{r}_{\mathrm{H}}\left(\mathrm{F}^{1}(l)\right) \\
& =\mathrm{F}^{0}\left(\mathrm{r}_{\mathrm{L}}(l)\right) \\
& =\mathrm{F}^{0}\left(\mathrm{r}_{\mathbf{X}}(x)\right) \\
& =\mathrm{E}^{0}(\mathrm{~s} \times(x)) \\
& =\mathrm{E}^{0}\left(\mathrm{r}_{\mathrm{R}}\left(\left[x ; l ; x_{1}\right]\right)\right)
\end{aligned}
$$

for all $x, x_{1} \in \mathrm{X}, l \in \mathrm{L}^{1}$ with $\mathrm{r}_{\mathrm{L}}(l)=\mathrm{r}_{\mathbf{X}}(x)$ and $\mathrm{s}_{\mathrm{L}}(l)=\mathrm{r}_{\mathbf{X}}\left(x_{1}\right)$. They intertwine the multiplication maps because

$$
\begin{aligned}
\mathrm{E}^{1}\left(\left[x ; l ; x_{1}\right] \cdot\left[x^{\prime} ; l^{\prime} ; x_{1}^{\prime}\right]\right) & =\mathrm{E}^{1}\left(\left[x ; l \cdot g \cdot l^{\prime} ; x_{1}^{\prime}\right]\right) \\
& =\mathrm{F}^{1}\left(l \cdot g \cdot l^{\prime}\right) \\
& =\mathrm{F}^{1}(l) \cdot \mathrm{F}^{1}\left(l^{\prime}\right) \\
& =\mathrm{E}^{1}\left(\left[x ; l ; x_{1}\right]\right) \cdot \mathrm{E}^{1}\left(\left[x^{\prime} ; l^{\prime} ; x_{1}^{\prime}\right]\right)
\end{aligned}
$$

for all $x, x_{1}, x^{\prime}, x_{1}^{\prime} \in \mathrm{X}, l, l^{\prime} \in \mathrm{L}^{1}$ with $\mathrm{r}_{\mathbf{X}}(x)=\mathrm{r}_{\mathrm{L}}(l), \mathrm{r}_{\mathbf{X}}\left(x_{1}\right)=\mathrm{s}_{\mathrm{L}}(l), \mathrm{r}_{\mathbf{X}}\left(x^{\prime}\right)=\mathrm{r}_{\mathrm{L}}\left(l^{\prime}\right)$, $r_{\mathrm{X}}\left(x_{1}^{\prime}\right)=\mathrm{s}_{\mathrm{L}}\left(l^{\prime}\right)$ and $\mathrm{s}_{\mathrm{X}}\left(x_{1}\right)=\mathrm{s}_{\mathrm{X}}\left(x^{\prime}\right)$. So the pair $\mathrm{E}=\left(\mathrm{E}^{1} ; \mathrm{E}^{0}\right)$ intertwines the source, range and multiplication maps and therefore, $\mathrm{E}: \mathrm{R} \rightarrow \mathrm{H}$ is a functor. We have to show that the arrow

$$
\mathrm{X} \times \times_{\mathrm{G}} \mathrm{L}^{1} \times_{\mathrm{G}} \mathrm{X} \stackrel{\left(\mathrm{E}^{1} ; \mathrm{s}_{\mathrm{R}}\right)}{\longrightarrow} \mathrm{H}^{1} \times_{\mathrm{SH}_{\mathrm{H}}, \mathrm{H}^{0}, \mathrm{E}^{0}} \mathrm{~K}^{0}
$$

is a cover. Consider the following diagram of pull-back squares:

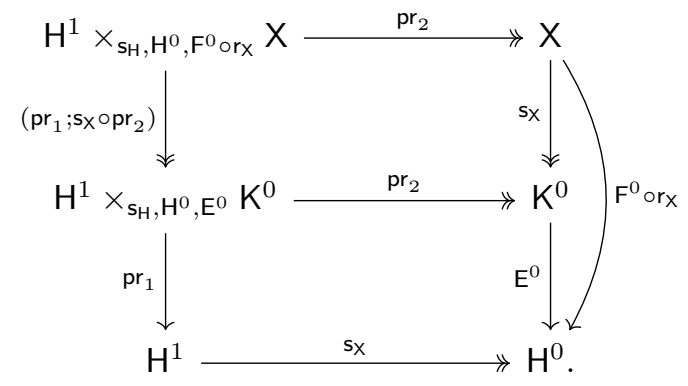

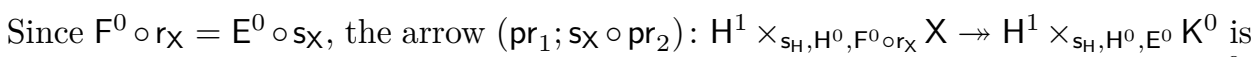
well-defined, and it is a cover because it is pull-back of the anchor map $s_{X}: X \rightarrow K^{0}$. Consider one more diagram of pull-back squares:

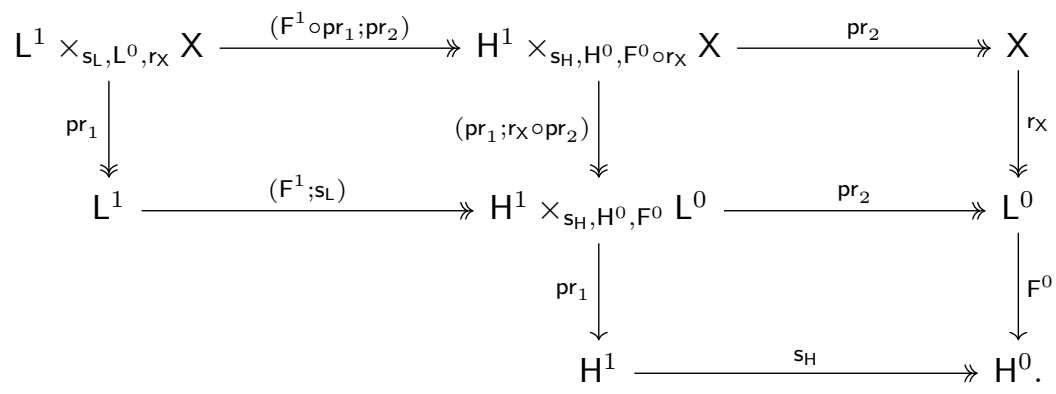

The diagram shows that the arrow $\left(\mathrm{F}^{1} \circ \mathrm{pr}_{1} ; \mathrm{pr}_{2}\right): \mathrm{L}^{1} \times_{\mathrm{S}_{\mathrm{L}}, \mathrm{L}^{0}, \mathrm{rX}_{\mathrm{X}}} \mathrm{X} \rightarrow \mathrm{H}^{1} \times_{\mathrm{s}_{\mathrm{H}}, \mathrm{H}^{0}, \mathrm{~F}^{0} \circ \mathrm{r}_{\mathrm{X}} \mathrm{X}}$

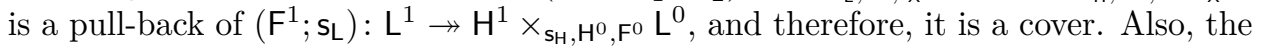

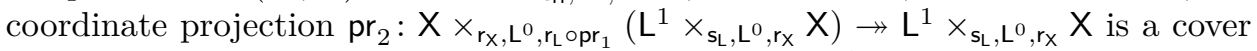
because it is a pull-back of the anchor map $r_{X}: X \rightarrow L^{0}$. So we have three covers and therefore, their composition

$\left(\mathrm{pr}_{1} ; \mathrm{sX} \circ \mathrm{pr}_{2}\right) \circ\left(\mathrm{F}^{1} \circ \mathrm{pr}_{1} ; \mathrm{pr}_{2}\right) \circ \mathrm{pr}_{2} \equiv \phi: \mathrm{X} \times_{\mathrm{rX}_{\mathrm{X}} \mathrm{L}^{0}, \mathrm{r}_{\mathrm{L}} \circ \mathrm{pr}_{1}}\left(\mathrm{~L}^{1} \times_{\mathrm{s}_{\mathrm{L}}, \mathrm{L}^{0}, \mathrm{r}_{\mathrm{X}}} \mathrm{X}\right) \rightarrow \mathrm{H}^{1} \times_{\mathrm{S}_{\mathrm{H}}, \mathrm{H}^{0}, \mathrm{E}^{0}} \mathrm{~K}^{0}$ 
is a cover by Assumption 2.14. This composition is defined elementwise by $\phi\left(x ;\left(l ; x_{1}\right)\right)=\left(\mathrm{F}^{1}(l) ; \mathrm{s}_{\mathbf{X}}\left(x_{1}\right)\right)$. Let us check that this composition is G-invariant in the sense of the $\mathrm{G}$-action on $\mathrm{X} \times_{\mathrm{rX}_{\mathrm{X}} \mathrm{L}^{0}, \mathrm{r}_{\mathrm{L}} \text { opr }}\left(\mathrm{L}^{1} \times_{\left.\mathrm{SL}_{\mathrm{L}}, \mathrm{L}^{0}, \mathrm{rX}_{\mathrm{X}} \mathrm{X}\right)}\right.$ defined elementwise by $\left(x ;\left(l ; x_{1}\right)\right) \cdot g=\left(x ;\left(l \cdot g ; g^{-1} \cdot x_{1}\right)\right)$. We have

$$
\begin{aligned}
\phi\left(\left(x ;\left(l ; x_{1}\right)\right) \cdot g\right) & =\phi\left(x ;\left(l \cdot g ; g^{-1} \cdot x_{1}\right)\right) \\
& =\left(\mathrm{F}^{1}(l \cdot g) ; \mathrm{s}_{\mathbf{X}}\left(g^{-1} \cdot x_{1}\right)\right) \\
& =\left(\mathrm{F}^{1}(l) ; \mathrm{s}_{\mathbf{X}}\left(x_{1}\right)\right) \\
& =\phi\left(x ;\left(l ; x_{1}\right)\right) .
\end{aligned}
$$

Therefore, there is a unique arrow $\phi^{\prime}: \mathrm{X} \times_{\mathrm{r}_{\mathrm{X}}, \mathrm{L}^{0}, \mathrm{r}_{2}}\left(\mathrm{~L}^{1} \times_{\mathrm{G}} \mathrm{X}\right) \rightarrow \mathrm{H}^{1} \times_{\mathrm{s}_{\mathrm{H}}, \mathrm{H}^{0}, \mathrm{E}^{0}} \mathrm{~K}^{0}$ such that $\phi^{\prime} \circ\left(\mathrm{pr}_{1} ; \mathrm{p}_{1}^{\prime} \circ \mathrm{pr}_{2}\right)=\phi$. Elementwise $\phi^{\prime}\left(x ;\left[l ; x_{1}\right]\right)=\left(\mathrm{F}^{1}(l) ; \mathrm{s}_{\mathbf{X}}\left(x_{1}\right)\right)$. Since the arrows $\left(\mathrm{pr}_{1} ; \mathrm{p}_{1}^{\prime} \circ \mathrm{pr}_{2}\right)$ and $\phi$ are covers, the arrow $\phi^{\prime}$ is a cover by Assumption 2.19. The cover $\phi^{\prime}$ is G-invariant in the sense of the G-action on $\mathrm{X} \times{ }_{\mathrm{r}_{\mathrm{X}}, \mathrm{L}^{0}, \mathrm{r}_{\mathrm{L}} \circ \mathrm{pr}_{1}}\left(\mathrm{~L}^{1} \times{ }_{\mathrm{G}} \mathrm{X}\right)$ defined elementwise by $\left(x ;\left[l ; x_{1}\right]\right) \cdot g=\left(g^{-1} \cdot x ;\left[g^{-1} \cdot l ; x_{1}\right]\right)$ because

$$
\begin{aligned}
\phi^{\prime}\left(\left(x ;\left[l ; x_{1}\right]\right) \cdot g\right) & =\phi^{\prime}\left(g^{-1} \cdot x ;\left[g^{-1} \cdot l ; x_{1}\right]\right) \\
& =\left(\mathrm{F}^{1}\left(g^{-1} \cdot l\right) ; \mathrm{s}_{x}\left(x_{1}\right)\right) \\
& =\left(\mathrm{F}^{1}(l) ; \mathrm{s}_{\times}\left(x_{1}\right)\right) \\
& =\phi^{\prime}\left(x ;\left(l ; x_{1}\right)\right) .
\end{aligned}
$$

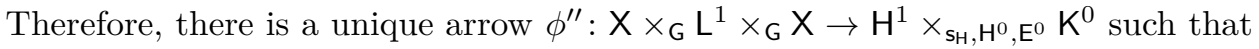
$\phi^{\prime \prime} \circ \mathrm{p}_{3}^{\prime}=\phi^{\prime}$. Elementwise $\phi^{\prime \prime}\left(\left[x ; l ; x_{1}\right]\right)=\phi^{\prime}\left(x ;\left[l ; x_{1}\right]\right)$. Since the arrows $\mathrm{p}_{3}^{\prime}$ and $\phi^{\prime}$ are covers, the arrow $\phi^{\prime \prime}$ is a cover by Assumption 2.19. For any element

$$
\left(x ;\left(l ; x_{1}\right)\right) \in \mathrm{X} \times_{\mathrm{r}_{\mathrm{X}}, \mathrm{L}^{0}, \mathrm{r}_{\mathrm{L}} \mathrm{opr} r_{1}}\left(\mathrm{~L}^{1} \times_{\mathrm{s}_{\mathrm{L}}, \mathrm{L}^{0}, \mathrm{r}_{\mathrm{X}}} \mathrm{X}\right)
$$

we have $\phi^{\prime \prime}\left(\left[x ; l ; x_{1}\right]\right)=\phi^{\prime}\left(x ;\left[l ; x_{1}\right]\right)=\left(\mathrm{F}^{1}(l) ; \mathrm{s}_{\mathbf{X}}\left(x_{1}\right)\right)=\left(\mathrm{E}^{1}\left(\left[x ; l ; x_{1}\right]\right) ; \mathrm{s}_{\mathrm{R}}\left(\left[x ; l ; x_{1}\right]\right)\right.$. Since the arrow $\mathrm{p}_{3}^{\prime} \circ\left(\mathrm{pr}_{1} ; \mathrm{p}_{1}^{\prime} \circ \mathrm{pr}_{2}\right)$ is a cover and therefore, it is epic, we have that $\phi^{\prime \prime}=\left(\mathrm{E}^{1} ; \mathrm{s}_{\mathrm{R}}\right)$. Hence the arrow $\left(\mathrm{E}^{1} ; \mathrm{s}_{\mathrm{R}}\right)$ is a cover and therefore, the functor $\mathrm{E}: \mathrm{R} \rightarrow \mathrm{H}$ is a groupoid fibration.

The next step is to prove that the fibre of the groupoid fibration $E: R \rightarrow H$ is isomorphic to the groupoid $\mathrm{K}$. Consider the following commuting square:

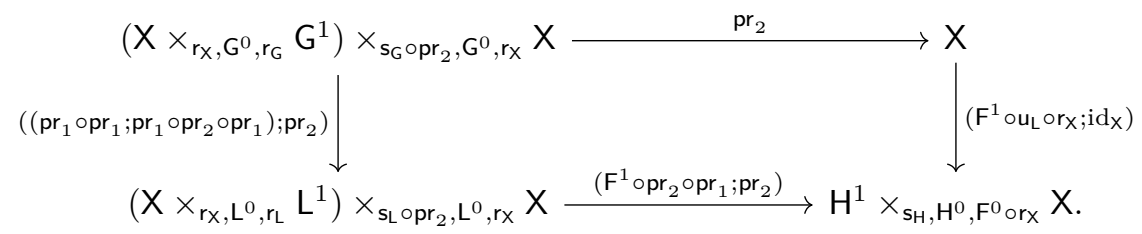

Our goal is to prove that this square is a pull-back square. Consider any arrows

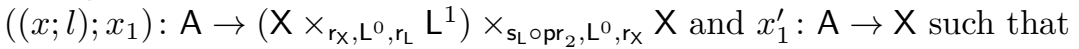

$$
\left(\mathrm{F}^{1}(l) ; x_{1}\right)=\left(1_{\mathrm{F}^{0}\left(\mathrm{rx}\left(x_{1}^{\prime}\right)\right)} ; x_{1}^{\prime}\right) \text {. }
$$

This gives that $x_{1}^{\prime}=x_{1}$ and $\mathrm{F}^{1}(l)=1_{\mathrm{F}^{0}}\left(\mathrm{rx}_{x}\left(x_{1}\right)\right)$. Therefore, the element $l: \mathrm{A} \rightarrow \mathrm{L}^{1}$ uniquely gives the element in $\left(l ; \mathrm{s}_{\mathrm{L}}(l)\right): \mathrm{A} \rightarrow \mathrm{G}^{1}$ by Definition 4.13 . So we have a

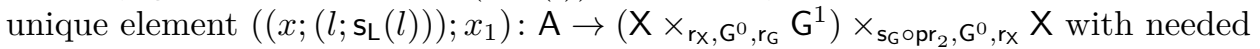
requirements. Therefore, the diagram above is a pull-back square.

The arrow $\left(\mathrm{F}^{1} \circ \mathrm{pr}_{2} \circ \mathrm{pr}_{1} ; \mathrm{pr}_{2}\right)$ from the diagram is G-invariant in the sense of the G-action on $\left(X \times_{r_{X}, \mathrm{~L}^{0}, r_{\mathrm{L}}} \mathrm{L}^{1}\right) \times_{\mathrm{S}_{\mathrm{L}} \mathrm{opr}_{2}, \mathrm{~L}^{0}, \mathrm{rX}_{\mathrm{X}}} \mathrm{X}$ considered above because

$$
\begin{aligned}
\left(\mathrm{F}^{1} \circ \mathrm{pr}_{2} \circ \mathrm{pr}_{1} ; \mathrm{pr}_{2}\right)\left(\left((x ; l) ; x_{1}\right) \cdot g\right) & =\left(\mathrm{F}^{1} \circ \mathrm{pr}_{2} \circ \mathrm{pr}_{1} ; \mathrm{pr}_{2}\right)\left(\left(g^{-1} \cdot x ; g^{-1} \cdot l\right) ; x_{1}\right) \\
& =\left(\mathrm{F}^{1}\left(g^{-1} \cdot l\right) ; x_{1}\right) \\
& =\left(\mathrm{F}^{1}(l) ; x_{1}\right) \\
& =\left(\mathrm{F}^{1} \circ \mathrm{pr}_{2} \circ \mathrm{pr}_{1} ; \mathrm{pr}_{2}\right)\left((x ; l) ; x_{1}\right) .
\end{aligned}
$$




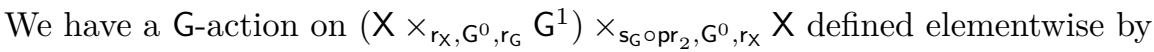

$$
\left.\left(\left(x ; g_{1}\right) ; x_{1}\right) \cdot g\right)=\left(\left(g^{-1} \cdot x ; g^{-1} \cdot g_{1}\right) ; x_{1}\right),
$$

and it is clear that the right side arrow on the diagram is a G-map. Therefore, there is a pull-back square

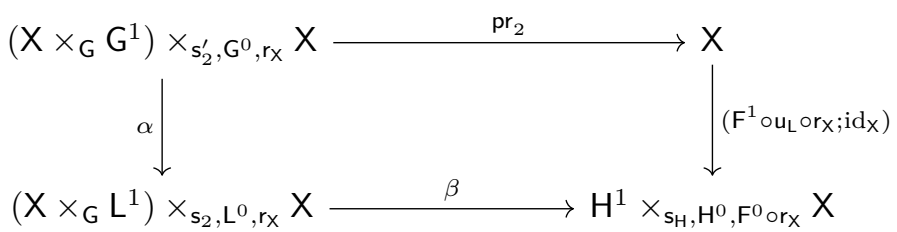

by Proposition 5.44 and Lemma 5.50 where the arrows $\alpha$ and $\beta$ are induced arrows defined elementwise by $\alpha\left([x ; g] ; x_{1}\right)=\left([x ; g] ; x_{1}\right)$ and $\beta\left([x ; l] ; x_{1}\right)=\left(\mathrm{F}^{1}(l) ; x_{1}\right)$. Now we have a diagram of pull-back squares with suitable G-actions on all the objects such that each arrow is a G-map. Therefore, the following diagram

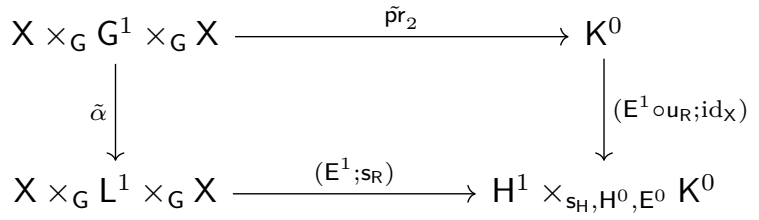

is a pull-back square by Lemma 5.65. This pull-back square gives the fibre of the groupoid fibration $E: R \rightarrow H$. The object $X \times{ }_{G} G^{1} \times_{G} X$ is the object of the composition of bibundle actors $\mathbb{X} \circ \mathbb{G}^{1} \circ \mathbb{X} .^{-1}$ We know that

$$
\mathbb{X} \circ \mathbb{G}^{1} \circ \mathbb{X}^{-1} \cong \mathbb{X} \circ \mathbb{X}^{-1} \cong \mathbb{K}^{1} \text {. }
$$

Therefore, we have a natural isomorphism $X \times_{G} G^{1} \times_{G} X \stackrel{\sim}{\rightarrow} K^{1}$, which gives the isomorphism between the groupoid $K$ and the fibre of $E: R \rightarrow H$.

The last step is to show that the groupoids $L$ and $R$ are equivalent. Consider the object $\mathrm{X} \times{ }_{\mathrm{G}} \mathrm{L}^{1}$ and define the left $\mathrm{R}$-action and right $\mathrm{L}$-action on it. We know that the multiplication map $\mathrm{m}_{\mathrm{L}}$ induces the $\mathrm{K}, \mathrm{G}$-map between bibundle actors $\mathbb{L}^{1} \circ \mathbb{L}^{1} \circ \mathbb{X}^{-1}$ and $\mathbb{L}^{1} \circ \mathbb{X}^{-1}$. That is $m_{1 *}^{\prime}: X \times_{G} L^{1} \times_{G} L^{1} \rightarrow X \times_{G} L^{1}$, defined elementwise by $\mathrm{m}_{1 *}^{\prime}\left(\left[l_{1} ; l ; x\right]\right)=\left[l_{1} \cdot l ; x\right]$. This map, with the suitable orbit space projection gives an action map of the groupoid $L$ on the object $X \times_{G} L^{1}$. That is

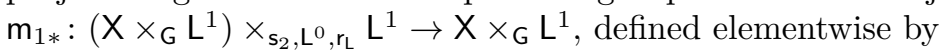

$$
[x ; l] \cdot l_{1}=\left[x ; l \cdot l_{1}\right]
$$

for all $x \in \mathrm{X}, l, l_{1} \in \mathrm{L}^{1}$ with $\mathrm{r}_{\mathrm{X}}(x)=\mathrm{r}_{\mathrm{L}}(l)$ and $\mathrm{s}_{\mathrm{L}}(l)=\mathrm{r}_{\mathrm{L}}\left(l_{1}\right)$. We have to show that $\left(\mathrm{X} \times{ }_{\mathrm{G}} \mathrm{L}^{1} ; \mathrm{m}_{1 *} ; \mathrm{s}_{2}\right)$ is a right $\mathrm{L}$-action. We have

$$
\begin{aligned}
\mathrm{s}_{2}\left([x ; l] \cdot l_{1}\right) & =\mathrm{s}_{2}\left(\left[x ; l \cdot l_{1}\right]\right) \\
& =\mathrm{s}_{\mathrm{L}}\left(l \cdot l_{1}\right) \\
& =\mathrm{s}_{\mathrm{L}}\left(l_{1}\right),
\end{aligned}
$$

also,

$$
\begin{aligned}
{[x ; l] \cdot\left(l_{1} \cdot l_{2}\right) } & =\left[x ; l \cdot\left(l_{1} \cdot l_{2}\right)\right] \\
& =\left[x ;\left(l \cdot l_{1}\right) \cdot l_{2}\right] \\
& =\left[x ; l \cdot l_{1}\right] \cdot l_{2} \\
& =\left([x ; l] \cdot l_{1}\right) \cdot l_{2},
\end{aligned}
$$

and

$$
\begin{aligned}
{[x ; l] \cdot 1_{\mathrm{s}_{\mathrm{L}}(l)} } & =\left[x ; l \cdot 1_{\mathrm{s}_{\mathrm{L}}(l)}\right] \\
& =[x ; l]
\end{aligned}
$$


for all $x \in \mathrm{X}, l, l_{1}, l_{2} \in \mathrm{L}^{1}$ with $\mathrm{r}_{\mathrm{X}}(x)=\mathrm{r}_{\mathrm{L}}(l), \mathrm{s}_{\mathrm{L}}(l)=\mathrm{r}_{\mathrm{L}}\left(l_{1}\right)$ and $\mathrm{s}_{\mathrm{L}}\left(l_{1}\right)=\mathrm{r}_{\mathrm{L}}\left(l_{2}\right)$. Therefore, $\left(X \times_{G} \mathrm{~L}^{1} ; \mathrm{m}_{1 *} ; \mathrm{s}_{2}\right)$ is a right $\mathrm{L}$-action by Lemma 5.7

We have the following natural isomorphisms of bibundle actors:

$$
\begin{aligned}
\mathbb{L}^{1} \circ \mathbb{X}^{-1} \circ \mathbb{X} \circ \mathbb{L}^{1} \circ \mathbb{X}^{-1} & \cong \mathbb{L}^{1} \circ \mathbb{G}^{1} \circ \mathbb{L}^{1} \circ \mathbb{X}^{-1} \\
& \cong \mathbb{L}^{1} \circ \mathbb{L}^{1} \circ \mathbb{X}^{-1}
\end{aligned}
$$

These isomorphisms and $\mathrm{m}_{1 *}^{\prime}$ give a $\mathrm{G}, \mathrm{K}$-map from $\mathbb{L}^{1} \circ \mathbb{X}^{-1} \circ \mathbb{X} \circ \mathbb{L}^{1} \circ \mathbb{X}^{-1}$ to $\mathbb{L}^{1} \circ \mathbb{X}^{-1}$. That is $\mathrm{m}_{2 *}:\left(\mathrm{X} \times \times_{\mathrm{G}} \mathrm{L}^{1} \times_{\mathrm{G}} \mathrm{X}\right) \times_{\mathrm{K}}\left(\mathrm{X} \times \times_{\mathrm{G}} \mathrm{L}^{1}\right) \rightarrow \mathrm{X} \times{ }_{\mathrm{G}} \mathrm{L}^{1}$ defined elementwise by $\mathrm{m}_{2 *}^{\prime}\left(\left[\left[x ; l ; x_{1}\right] ;\left[x_{1}^{\prime} ; l^{\prime}\right]\right]\right)=\left[x ; l \cdot g \cdot l^{\prime}\right]$. This map with the suitable orbit space projection gives an action map of the groupoid $R$ on the object $X \times_{G} L^{1}$. That is $\mathrm{m}_{2 *}^{\prime}:\left(\mathrm{X} \times_{\mathrm{G}} \mathrm{L}^{1} \times_{\mathrm{G}} \mathrm{X}\right) \times_{\mathrm{s}_{\mathrm{R}}, \mathrm{K}^{0}, \mathrm{r}_{\mathrm{R}}^{\prime}} \mathrm{X} \times \times_{\mathrm{G}} \mathrm{L}^{1}$ defined elementwise by

$$
\left[x ; l ; x_{1}\right] \cdot\left[x_{1}^{\prime} ; l^{\prime}\right]=\left[x ; l \cdot g \cdot l^{\prime}\right]
$$

for all $x, x_{1}, x_{1}^{\prime} \in \mathrm{X}, l, l^{\prime} \in \mathrm{L}^{1}$ with $\mathrm{r}_{\mathrm{X}}(x)=\mathrm{r}_{\mathrm{L}}(l), \mathrm{r}_{\mathrm{X}}\left(x_{1}\right)=\mathrm{s}_{\mathrm{L}}(l), \mathrm{r}_{\mathrm{X}}\left(x_{1}^{\prime}\right)=\mathrm{r}_{\mathrm{L}}\left(l^{\prime}\right)$ and $\mathrm{s}_{\mathrm{X}}\left(x_{1}\right)=\mathrm{s}_{\mathrm{X}}\left(x_{1}^{\prime}\right)$, where $g \in \mathrm{G}^{1}$ is the element which is given by the following composition $\mathrm{pr}_{1} \circ\left(\mathrm{pr}_{1} ; \mathrm{m}_{1 \mathrm{X}}\right)^{-1} \circ\left(x_{1} ; x_{1}^{\prime}\right)$. We have to show that $\left(\mathrm{X} \times{ }_{\mathrm{G}} \mathrm{L}^{1} ; \mathrm{m}_{2 *} ; \mathrm{r}_{\mathrm{R}}^{\prime}\right)$ is a left R-action. We have

$$
\begin{aligned}
\mathrm{r}_{\mathrm{R}}^{\prime}\left(\left[x ; l ; x_{1}\right] \cdot\left[x_{1}^{\prime} ; l^{\prime}\right]\right) & =\mathrm{r}_{\mathrm{R}}^{\prime}\left(\left[x ; l \cdot g \cdot l^{\prime}\right]\right) \\
& =\mathrm{sX}_{\mathrm{X}}(x)=\mathrm{r}_{\mathrm{R}}\left(\left[x ; l ; x_{1}\right]\right)
\end{aligned}
$$

also,

$$
\begin{aligned}
\left(\left[\hat{x} ; \hat{l} ; \hat{x}_{1}\right] \cdot\left[x ; l ; x_{1}\right]\right) \cdot\left[x_{1}^{\prime} ; l^{\prime}\right] & =\left[\hat{x} ; \hat{l} \cdot g^{\prime} \cdot l ; x_{1}\right] \cdot\left[x_{1}^{\prime} ; l^{\prime}\right] \\
& =\left[\hat{x} ; \hat{l} \cdot g^{\prime} \cdot l \cdot g \cdot l^{\prime}\right] \\
& =\left[\hat{x} ; \hat{l} ; \hat{x}_{1}\right] \cdot\left[x ; l \cdot g \cdot l^{\prime}\right] \\
& =\left[\hat{x} ; \hat{l} ; \hat{x}_{1}\right] \cdot\left(\left[x ; l ; x_{1}\right] \cdot\left[x_{1}^{\prime} ; l^{\prime}\right]\right),
\end{aligned}
$$

and

$$
\begin{aligned}
1_{\mathrm{s}\left(x_{1}^{\prime}\right)} \cdot\left[x_{1}^{\prime} ; l^{\prime}\right] & =\left[x_{1}^{\prime} ; 1_{\mathrm{r}_{\mathrm{X}}\left(x_{1}^{\prime}\right)} ; x_{1}^{\prime}\right] \cdot\left[x_{1}^{\prime} ; l^{\prime}\right] \\
& =\left[x_{1}^{\prime} ; 1_{\mathrm{r}_{\mathrm{X}}\left(x_{1}^{\prime}\right)} \cdot 1_{\mathrm{r}_{\mathrm{X}}\left(x_{1}^{\prime}\right)} \cdot l^{\prime}\right] \\
& =\left[x_{1}^{\prime} ; l^{\prime}\right]
\end{aligned}
$$

for all $x, x_{1}, x_{1}^{\prime}, \hat{x}, \hat{x}_{1} \in \mathrm{X}, l, l^{\prime}, \hat{l} \in \mathrm{L}^{1}$ with $\mathrm{r}_{\mathbf{X}}(x)=\mathrm{r}_{\mathrm{L}}(l), \mathrm{r}_{\mathbf{X}}\left(x_{1}\right)=\mathrm{s}_{\mathrm{L}}(l), \mathrm{r}_{\mathbf{X}}(\hat{x})=\mathrm{r}_{\mathrm{L}}(\hat{l})$, $\mathrm{r}_{\mathbf{X}}\left(\hat{x}_{1}\right)=\mathrm{s}_{\mathrm{L}}(\hat{l}), \mathrm{r}_{\mathbf{X}}\left(x_{1}^{\prime}\right)=\mathrm{r}_{\mathrm{L}}\left(l^{\prime}\right), \mathrm{s}_{\mathbf{X}}\left(x_{1}\right)=\mathrm{s}_{\mathbf{X}}\left(x_{1}^{\prime}\right)$ and $\mathrm{s}_{\mathbf{X}}\left(\hat{x}_{1}\right)=\mathrm{s}_{\mathbf{X}}(x)$, where $g$ and $g^{\prime}$ are elements in $\mathrm{G}^{1}$ which are given by the pairs $\left(x_{1} ; x_{1}^{\prime}\right)$ and $\left(\hat{x}_{1} ; x\right)$, respectively. Therefore, $\left(\mathrm{X} \times{ }_{\mathrm{G}} \mathrm{L}^{1} ; \mathrm{m}_{2 *} ; \mathrm{r}_{\mathrm{R}}^{\prime}\right)$ is a left R-action by Remark 5.11. We are going to show that $\left(\mathrm{X} \times{ }_{\mathrm{G}} \mathrm{L}^{1} ; \mathrm{m}_{1 *} ; \mathrm{m}_{2 *} ; \mathrm{s}_{2} ; \mathrm{r}_{\mathrm{R}}^{\prime}\right)$ defines an equivalence from $\mathrm{R}$ to $\mathrm{L}$. Firstly, we have to check that it is an R, L-bibundle. We have

$$
\begin{aligned}
\mathrm{s}_{2}\left(\left[x ; l ; x_{1}\right] \cdot\left[x_{1}^{\prime} ; l^{\prime}\right]\right) & =\mathrm{s}_{2}\left(\left[x ; l \cdot g \cdot l^{\prime}\right]\right) \\
& =\mathrm{s}_{\mathrm{L}}\left(l \cdot g \cdot l^{\prime}\right) \\
& =\mathrm{s}_{\mathrm{L}}\left(l^{\prime}\right) \\
& =\mathrm{s}_{2}\left(\left[x_{1}^{\prime} ; l^{\prime}\right]\right),
\end{aligned}
$$

also,

$$
\begin{aligned}
\mathrm{r}_{\mathrm{R}}^{\prime}\left(\left[x_{1}^{\prime} ; l^{\prime}\right] \cdot l^{\prime \prime}\right) & =\mathrm{r}_{\mathrm{R}}^{\prime}\left(\left[x_{1}^{\prime} ; l^{\prime} \cdot l^{\prime \prime}\right]\right) \\
& =\mathrm{s}_{\mathbf{X}}\left(x_{1}^{\prime}\right) \\
& =\mathrm{r}_{\mathrm{R}}^{\prime}\left(\left[x_{1}^{\prime} ; l^{\prime}\right]\right),
\end{aligned}
$$


and

$$
\begin{aligned}
\left(\left[x ; l ; x_{1}\right] \cdot\left[x_{1}^{\prime} ; l^{\prime}\right]\right) \cdot l^{\prime \prime} & =\left[x ; l \cdot g \cdot l^{\prime}\right] \cdot l^{\prime \prime} \\
& =\left[x ; l \cdot g \cdot l^{\prime} \cdot l^{\prime \prime}\right] \\
& =\left[x ; l ; x_{1}\right] \cdot\left[x_{1}^{\prime} ; l^{\prime} \cdot l^{\prime \prime}\right] \\
& =\left[x ; l ; x_{1}\right] \cdot\left(\left[x_{1}^{\prime} ; l^{\prime}\right] \cdot l^{\prime \prime}\right)
\end{aligned}
$$

for all $x, x_{1}, x_{1}^{\prime} \in \mathrm{X}, l, l^{\prime}, l^{\prime \prime} \in \mathrm{L}^{1}$ with $\mathrm{r}_{\mathbf{X}}(x)=\mathrm{r}_{\mathrm{L}}(l), \mathrm{r}_{\mathbf{X}}\left(x_{1}\right)=\mathrm{s}_{\mathrm{L}}(l), \mathrm{r}_{\mathbf{X}}\left(x_{1}^{\prime}\right)=\mathrm{r}_{\mathrm{L}}\left(l^{\prime}\right)$, $\mathrm{s}_{\mathrm{L}}\left(l^{\prime}\right)=\mathrm{r}_{\mathrm{L}}\left(l^{\prime}\right)$ and $\mathrm{s}_{\mathrm{X}}\left(x_{1}\right)=\mathrm{s}_{\mathrm{X}}\left(x_{1}^{\prime}\right)$, where $g \in \mathrm{G}^{1}$ is the element considered above. Therefore, $\left(\mathrm{X} \times{ }_{\mathrm{G}} \mathrm{L}^{1} ; \mathrm{m}_{1 *} ; \mathrm{m}_{2 *} ; \mathrm{s}_{2} ; \mathrm{r}_{\mathrm{R}}^{\prime}\right)$ is an $\mathrm{R}$, L-bibundle. We have checked above that the anchor maps $s_{2}$ and $r_{R}^{\prime}$ are covers. After this, we have to show that the following arrows

$$
\left(X \times_{G} L^{1}\right) \times_{s_{2}, L^{0}, r_{L}} L^{1} \stackrel{\left(p r_{1} ; m_{2 *}\right)}{\longrightarrow}\left(X \times_{G} L^{1}\right) \times_{r_{R}^{\prime}, K^{0}, r_{R}^{\prime}}\left(X \times_{G} L^{1}\right)
$$

and

$$
\left(X \times_{G} L^{1} \times_{G} X\right) \times_{s_{R}, K^{0}, r_{R}^{\prime}}\left(X \times_{G} L^{1}\right) \stackrel{\left(m_{1 *} ; r_{2}\right)}{\longrightarrow}\left(X \times_{G} L^{1}\right) \times_{s_{2}, L^{0}, s_{2}}\left(X \times_{G} L^{1}\right)
$$

are invertible. We know that the fibre product of the G-map pr $r_{1}: X \times_{r_{X}, L^{0}, r_{L}} L^{1} \rightarrow X$ on itself is a principal G-bundle over

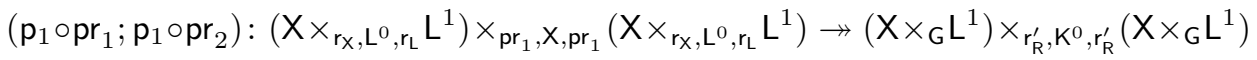

by Lemma 5.65. Also, the pull-back of the G-bundle $\left(X \times_{r_{x}, L^{0}, r_{L}} L^{1} ; m_{1} ; r_{1}\right)$ over $\mathrm{s}_{\mathrm{L}} \circ \mathrm{pr}_{2}$ along the cover $\mathrm{r}_{\mathrm{L}}: \mathrm{L}^{1} \rightarrow \mathrm{L}^{0}$ is a principal G-bundle over

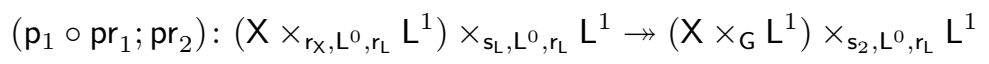

by Lemma 5.50 Let $\gamma$ be the arrow

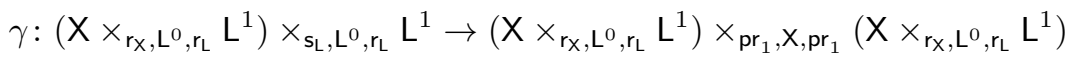

defined elementwise by $\gamma\left((x ; l) ; l_{1}\right)=\left((x ; l) ;\left(x ; l \cdot l_{1}\right)\right)$. It is a $\mathrm{G}$-map because

$$
\begin{aligned}
\gamma\left(\left((x ; l) ; l_{1}\right) \cdot g\right) & =\gamma\left((x ; l) \cdot g ; l_{1}\right) \\
& =\gamma\left(\left(g^{-1} \cdot x ; g^{-1} \cdot l\right) ; l_{1}\right) \\
& =\left(\left(g^{-1} \cdot x ; g^{-1} \cdot l\right) ;\left(g^{-1} \cdot x ; g^{-1} \cdot l \cdot l_{1}\right)\right) \\
& =\left((x ; l) \cdot g ;\left(x ; l \cdot l_{1}\right) \cdot g\right) \\
& =\left((x ; l) ;\left(x ; l \cdot l_{1}\right)\right) \cdot g \\
& =\gamma\left((x ; l) ; l_{1}\right) \cdot g
\end{aligned}
$$

for all $x \in \mathrm{X}, l, l_{1} \in \mathrm{L}^{1}, g \in \mathrm{G}^{1}$ with $\mathrm{rX}_{\mathbf{X}}(x)=\mathrm{r}_{\mathrm{L}}(l)$ and $\mathrm{s}_{\mathrm{L}}(l)=\mathrm{r}_{\mathrm{L}}\left(l_{1}\right)=\mathrm{r}_{\mathrm{G}}(g)$. Also, we have that

$$
\begin{aligned}
\left(\mathrm{pr}_{1} ; \mathrm{m}_{2 *}\right)\left(\left(\mathrm{p}_{1} \circ \mathrm{pr}_{1} ; \mathrm{pr}_{2}\right)\left((x ; l) ; l_{1}\right)\right) & =\left(\mathrm{pr}_{1} ; \mathrm{m}_{2 *}\right)\left([x ; l] ; l_{1}\right) \\
& =\left([x ; l] ;[x ; l] \cdot l_{1}\right) \\
& =\left([x ; l] ;\left[x ; l \cdot l_{1}\right]\right) \\
& =\left(\mathrm{p}_{1} \circ \mathrm{pr}_{1} ; \mathrm{p} \circ \mathrm{pr}_{2}\right)\left((x ; l) ;\left(x ; l \cdot l_{1}\right)\right) \\
& =\left(\mathrm{p}_{1} \circ \mathrm{pr}_{1} ; \mathrm{p} \circ \mathrm{pr}_{2}\right)\left(\gamma\left((x ; l) ; l_{1}\right)\right)
\end{aligned}
$$

for all $x \in \mathrm{X}, l, l_{1} \in \mathrm{L}^{1}$ with $\mathrm{rX}_{\mathrm{X}}(x)=\mathrm{r}_{\mathrm{L}}(l)$ and $\mathrm{s}_{\mathrm{L}}(l)=\mathrm{r}_{\mathrm{L}}\left(l_{1}\right)$. Hence we have that $\left(\mathrm{pr}_{1} ; \mathrm{m}_{2 *}\right) \circ\left(\mathrm{p}_{1} \circ \mathrm{pr}_{1} ; \mathrm{pr}_{2}\right)=\left(\mathrm{p}_{1} \circ \mathrm{pr}_{1} ; \mathrm{p} \circ \mathrm{pr}_{2}\right) \circ \gamma$ and therefore, the arrow $\left(\mathrm{pr}_{1} ; \mathrm{m}_{2 *}\right)$ is induced by $\gamma$. It is clear that $\gamma$ is invertible with $\gamma^{-1}$ defined elementwise by $\gamma^{-1}\left((x ; l) ;\left(x ; l_{1}\right)\right)=\left((x ; l) ; l^{-1} \cdot l_{1}\right)$ for all $x \in \mathrm{X}, l, l_{1} \in \mathrm{L}^{1}$ with $\mathrm{r}_{\mathrm{X}}(x)=\mathrm{r}_{\mathrm{L}}(l)=\mathrm{r}_{\mathrm{L}}\left(l_{1}\right)$. The composition $l^{-1} \cdot l_{1}$ is well-defined because $\mathrm{r}_{\mathrm{L}}(l)=\mathrm{r}_{\mathrm{L}}\left(l_{1}\right)$. Therefore, the arrow $\left(\mathrm{pr}_{1} ; \mathrm{m}_{2 *}\right)$ is invertible by Corollary 5.54 . So we have proved that the R, L-bibundle $\left(\mathrm{X} \times{ }_{\mathrm{G}} \mathrm{L}^{1} ; \mathrm{m}_{1 *} ; \mathrm{m}_{2 *} ; \mathrm{s}_{2} ; \mathrm{r}_{\mathrm{R}}^{\prime}\right)$ is a bibundle functor from $\mathrm{R}$ to $\mathrm{L}$. 
We also have to show that the arrow $\left(\mathrm{m}_{1 *} ; \mathrm{pr}_{2}\right)$ is invertible. We can do this by the following logical argumentation. Let us review and analyse our construction at this moment. We have had the groupoid fibration $F: L \rightarrow H$ with fibre $G$. We have used the groupoid equivalence $\mathbb{X}$ from $\mathrm{G}$ to $\mathrm{K}$ and have constructed the groupoid $\mathrm{R}$ with the object of the composition of bibundle actors $\mathbb{X} \circ \mathbb{L}^{1} \circ \mathbb{X}^{-1}$ as arrows. Also, we have constructed the bibundle functor from $R$ to $L$ which is defined on the object of the composition of bibundle actors $\mathbb{L}^{1} \circ \mathbb{X}^{-1}$. Now, the idea is the following. We have the groupoid fibration $\mathrm{E}: \mathrm{R} \rightarrow \mathrm{H}$ with fibre $\mathrm{K}$. We can use the groupoid equivalence $\mathbb{X}^{-1}$ from $K$ to $G$ and construct the groupoid $L^{\prime}$ with the object of the composition of bibundle actors $\mathbb{X}^{-1} \circ \mathbb{R}^{1} \circ \mathbb{X}$ as arrows. We have the following natural isomorphisms

$$
\begin{aligned}
\mathbb{X}^{-1} \circ \mathbb{R}^{1} \circ \mathbb{X} & \cong \mathbb{X}^{-1} \circ\left(\mathbb{X} \circ \mathbb{L}^{1} \circ \mathbb{X}^{-1}\right) \circ \mathbb{X} \\
& \cong\left(\mathbb{X}^{-1} \circ \mathbb{X}\right) \circ \mathbb{L}^{1} \circ\left(\mathbb{X}^{-1} \circ \mathbb{X}\right) \\
& \cong \mathbb{G}^{1} \circ \mathbb{L}^{1} \circ \mathbb{G}^{1} \\
& \cong \mathbb{L}^{1} .
\end{aligned}
$$

These isomorphisms show that the groupoid $\mathrm{L}^{\prime}$ is naturally isomorphic to the groupoid L. So we can go on to do the same steps and construct a bibundle functor from $L$ to $R$. This bibundle functor is defined on the object $X \times_{K} R^{1}$ which is given by the composition of bibundle actors $\mathbb{R}^{1} \circ \mathbb{X}$. We have natural isomorphisms

$$
\begin{aligned}
\mathbb{R}^{1} \circ \mathbb{X} & \cong\left(\mathbb{X} \circ \mathbb{L}^{1} \circ \mathbb{X}^{-1}\right) \circ \mathbb{X} \\
& \cong \mathbb{X} \circ \mathbb{L}^{1} \circ\left(\mathbb{X}^{-1} \circ \mathbb{X}\right) \\
& \cong \mathbb{X} \circ \mathbb{L}^{1} \circ \mathbb{G}^{1} \\
& \cong \mathbb{X} \circ \mathbb{L}^{1}
\end{aligned}
$$

The bibundle actor $\mathbb{X} \circ \mathbb{L}^{1}$ from $G$ to $\mathrm{K}$ is defined on the object which is an orbit space of the G-action on $\mathrm{L}^{1} \times_{\mathrm{SL}_{\mathrm{L}} \mathrm{L}^{0}, \mathrm{rX}} \mathrm{X}$ defined elementwise by $(l ; x) \cdot g=\left(l \cdot g ; g^{-1} \cdot x\right)$. The bibundle actor $\mathbb{L}^{1} \circ \mathbb{X}^{-1}$ from $\mathrm{K}$ to $\mathrm{G}$ is defined on the object which is an orbit space of the G-action on $\mathrm{X} \times{ }_{r_{X}, \mathrm{~L}^{0}, \mathrm{r}_{\mathrm{L}} \mathrm{L}^{1}}$ defined elementwise by $(x ; l) \cdot g=\left(g^{-1} \cdot x ; g^{-1} \cdot l\right)$.

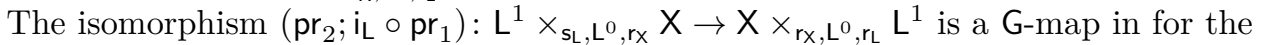
G-actions considered above because

$$
\begin{aligned}
\left(\mathrm{pr}_{2} ; \mathrm{i}_{\mathrm{L}} \circ \mathrm{pr}_{1}\right)((l ; x) \cdot g) & =\left(\mathrm{pr}_{2} ; \mathrm{i}_{\mathrm{L}} \circ \mathrm{pr}_{1}\right)\left(l \cdot g ; g^{-1} \cdot x\right) \\
& =\left(g^{-1} \cdot x ;(l \cdot g)^{-1}\right) \\
& =\left(g^{-1} \cdot x ; g^{-1} \cdot l^{-1}\right) \\
& =\left(x ; l^{-1}\right) \cdot g \\
& =\left(\mathrm{pr}_{2} ; \mathrm{i}_{\mathrm{L}} \circ \mathrm{pr}_{1}\right)(l ; x) \cdot g .
\end{aligned}
$$

Therefore, it induces an isomorphism $\tilde{\mathrm{I}}_{\mathrm{L}}: \mathrm{L}^{1} \times{ }_{\mathrm{G}} \mathrm{X} \stackrel{\sim}{\rightarrow} \mathrm{X} \times{ }_{\mathrm{G}} \mathrm{L}^{1}$, defined elementwise by $\tilde{\mathrm{i}}_{\mathrm{L}}([l ; x])=\left[x ; l^{-1}\right]$. The construction shows that the isomorphism $\mathbb{R}^{1} \circ \mathbb{X} \cong \mathbb{X} \circ \mathbb{L}^{1}$ equips the object $\mathrm{L}^{1} \times_{\mathrm{G}} \mathrm{X}$ with the left $\mathrm{L}$-action $\left(\mathrm{L}^{1} \times_{\mathrm{G}} \mathrm{X} ; \mathrm{m}_{2 *}^{\prime} ; \mathrm{s}_{2}^{\prime}\right)$ and right R-action $\left(\mathrm{L}^{1} \times_{\mathrm{G}} \mathrm{X} ; \mathrm{m}_{1 *}^{\prime} ; \mathrm{r}_{\mathrm{R}}^{\prime \prime}\right)$ defined elementwise by $l_{1} \cdot[l ; x]=\left[l_{1} \cdot l ; x\right]$ and by $[l ; x] \cdot\left[x^{\prime} ; l^{\prime}, x_{1}\right]=$ $\left[l \cdot g \cdot l^{\prime} ; x_{1}\right]$, respectively. This gives a bibundle functor from $\mathrm{L}$ to $\mathrm{R}$. So the arrow

$$
\left(\mathrm{L}^{1} \times_{\mathrm{G}} \mathrm{X}\right) \times_{\mathrm{r}_{\mathrm{R}}^{\prime \prime}, \mathrm{K}^{0}, \mathrm{r}_{\mathrm{R}}}\left(\mathrm{X} \times \times_{\mathrm{G}} \mathrm{L}^{1} \times_{\mathrm{G}} \mathrm{X}\right) \stackrel{\left(\mathrm{pr}_{1} ; \mathrm{m}_{1 *}^{\prime}\right)}{\longrightarrow}\left(\mathrm{L}^{1} \times_{\mathrm{G}} \mathrm{X}\right) \times_{\mathrm{s}_{2}^{\prime}, \mathrm{L}^{0}, \mathrm{~s}_{2}^{\prime}}\left(\mathrm{L}^{1} \times_{\mathrm{G}} \mathrm{X}\right)
$$

is invertible. Also, there are isomorphisms

$\left(X \times{ }_{G} L^{1} \times_{G} X\right) \times_{S_{R}, K^{0}, r_{R}^{\prime}}\left(X \times{ }_{G} L^{1}\right) \stackrel{\left(i_{L}^{-1} o p r_{2} ; i_{R} o p r_{1}\right)}{\longrightarrow}\left(L^{1} \times_{G} X\right) \times_{r_{R}^{\prime \prime}, K^{0}, r_{R}}\left(X \times_{G} L^{1} \times_{G} X\right)$

and

$$
\left(\mathrm{L}^{1} \times_{\mathrm{G}} \mathrm{X}\right) \times_{\mathrm{s}_{2}^{\prime}, \mathrm{L}^{0}, \mathrm{~s}_{2}^{\prime}}\left(\mathrm{L}^{1} \times_{\mathrm{G}} \mathrm{X}\right) \stackrel{\left(\tilde{\mathrm{i}}_{\mathrm{L}} \circ \mathrm{opr}_{2} ; \tilde{i}_{\mathrm{L}} \circ \mathrm{pr}_{1}\right)}{\longrightarrow}\left(\mathrm{X} \times \times_{\mathrm{G}} \mathrm{L}^{1}\right) \times_{\mathrm{s}_{2}, \mathrm{~L}^{0}, \mathrm{~s}_{2}}\left(\mathrm{X} \times{ }_{\mathrm{G}} \mathrm{L}^{1}\right) .
$$


We have

$$
\begin{aligned}
& \left(\left(\tilde{\mathrm{i}}_{\mathrm{L}} \circ \mathrm{pr}_{2} ; \tilde{\mathrm{i}}_{\mathrm{L}} \circ \mathrm{pr}_{1}\right) \circ\left(\mathrm{pr}_{1} ; \mathrm{m}_{1 *}^{\prime}\right) \circ\left(\tilde{\mathrm{i}}_{\mathrm{L}}^{-1} \circ \mathrm{pr}_{2} ; \mathrm{i}_{\mathrm{R}} \circ \mathrm{pr}_{1}\right)\right)\left(\left[x ; l ; x_{1}\right] ;\left[x_{2} ; l_{1}\right]\right) \\
= & \left(\left(\tilde{\mathrm{i}}_{\mathrm{L}} \circ \mathrm{pr}_{2} ; \tilde{\mathrm{i}}_{\mathrm{L}} \circ \mathrm{pr}_{1}\right) \circ\left(\mathrm{pr}_{1} ; \mathrm{m}_{1 *}^{\prime}\right)\right)\left(\left[l_{1}^{-1} ; x_{2}\right] ;\left[x_{1} ; l^{-1} ; x\right]\right) \\
= & \left(\tilde{\mathrm{i}}_{\mathrm{L}} \circ \mathrm{pr}_{2} ; \tilde{\mathrm{i}}_{\mathrm{L}} \circ \mathrm{pr}_{1}\right)\left(\left[l_{1}^{-1} ; x_{2}\right] ;\left[l_{1}^{-1} \cdot g^{-1} \cdot l^{-1} ; x\right]\right) \\
= & \left(\left[x ; l \cdot g \cdot l_{1}\right] ;\left[x_{2} ; l_{1}\right]\right) \\
= & \left(\left[x ; l ; x_{1}\right] \cdot\left[x_{2} ; l_{1}\right] ;\left[x_{2} ; l_{1}\right]\right) \\
= & \left(\mathrm{m}_{1 *} ; \mathrm{pr}_{2}\right)\left(\left[x ; l ; x_{1}\right] ;\left[x_{2} ; l_{1}\right]\right)
\end{aligned}
$$

for all $x, x_{1}, x_{2} \in \mathrm{X}, l, l_{1} \in \mathrm{L}^{1}$ with $\mathrm{r}_{\mathrm{X}}(x)=\mathrm{r}_{\mathrm{L}}(l), \mathrm{r}_{\mathrm{X}}\left(x_{1}\right)=\mathrm{s}_{\mathrm{L}}(l), \mathrm{r}_{\mathrm{X}}\left(x_{2}\right)=\mathrm{r}_{\mathrm{L}}\left(l_{1}\right)$ and $\mathrm{s}_{\mathrm{X}}\left(x_{1}\right)=\mathrm{s}_{\mathrm{X}}\left(x_{2}\right)$, where the element $g \in \mathrm{G}^{1}$ is given by the composition $\mathrm{pr}_{1} \circ\left(\mathrm{pr}_{1} ; \mathrm{m}_{1 \mathrm{X}}\right)^{-1} \circ\left(x_{1} ; x_{2}\right)$. It is clear that the composition $\mathrm{pr}_{1} \circ\left(\mathrm{pr}_{1} ; \mathrm{m}_{1 \mathrm{X}}\right)^{-1} \circ\left(x_{2} ; x_{1}\right)$ gives the element $g^{-1} \in \mathrm{G}^{1}$. Therefore, we have

$$
\left(\mathrm{m}_{1 *} ; \mathrm{pr}_{2}\right)=\left(\tilde{\mathrm{i}}_{\mathrm{L}} \circ \mathrm{pr}_{2} ; \tilde{\mathrm{i}}_{\mathrm{L}} \circ \mathrm{pr}_{1}\right) \circ\left(\mathrm{pr}_{1} ; \mathrm{m}_{1 *}^{\prime}\right) \circ\left(\tilde{\mathrm{i}}_{\mathrm{L}}^{-1} \circ \mathrm{pr}_{2} ; \mathrm{i}_{\mathrm{R}} \circ \mathrm{pr}_{1}\right) \text {. }
$$

Hence $\left(\mathrm{m}_{1 *} ; \mathrm{pr}_{2}\right)$ is an isomorphism and therefore, $\left(\mathrm{X} \times_{\mathrm{G}} \mathrm{L}^{1} ; \mathrm{m}_{1 *} ; \mathrm{m}_{2 *} ; \mathrm{s}_{2} ; \mathrm{r}_{\mathrm{R}}^{\prime}\right)$ is a groupoid equivalence from $R$ to $L$ and the proof is done.

\section{Examples of CATEgories With PARTial COVERS}

In this section, we discuss stronger pretopologies on different categories and check whether they satisfy our extra assumptions. For each case we check the conditions (1) (2) and (3) in Definition 2.1, and then we describe covers. We begin with a trivial examples on an arbitrary category with all fibre products.

Example 8.1. Let $\mathcal{C}$ be any category with all fibre products and let $\mathcal{F}_{p}$ be the class of all arrows in $\mathcal{C}$ and let $\mathcal{F}$ be the class of all coequalisers in $\mathcal{C}$. All conditions in Definition 2.1 are clearly satisfied.

In this general case we have not any additional information about the extra assumptions.

Example 8.2. Let $\mathcal{C}$ be any category with all fibre products and let $\mathcal{F}_{p}$ be the class of all monics in $\mathcal{C}$. Then $\left(\mathcal{C}, \mathcal{F}_{p}\right)$ is a category with partial covers. Since any isomorphism is monic, the condition (1) is satisfied. Since the composition of monics is monic, the condition (2) is satisfied. Consider situation as in (3) Assume $\mathrm{pr}_{2} \circ \alpha=\mathrm{pr}_{2} \circ \beta$ for some parallel arrows $\alpha, \beta$ : $\mathrm{B} \rightrightarrows \mathrm{A} \times_{f, B, x} \mathrm{X}$. Then $f \circ \mathrm{pr}_{1} \circ \alpha=x \circ \mathrm{pr}_{2} \circ \alpha=x \circ \mathrm{pr}_{2} \circ \beta=f \circ \mathrm{pr}_{1} \circ \beta$. Since $f$ is monic, $\mathrm{pr}_{1} \circ \alpha=\mathrm{pr}_{1} \circ \beta$. By the universality of a fibre product $\alpha=\beta$. Therefore, $\mathrm{pr}_{2}: \mathbf{A} \times_{f, B, x} \mathbf{X} \rightarrow \mathbf{X}$ is monic. So the condition (3) holds. The covers are the isomorphisms by Lemma 2.11

In the case of Example 8.2 the Assumption 2.14 is the following: The composition of isomorphisms is an isomorphism. This is clearly satisfied. Assumption 2.15 is satisfied too because the pull-back of any isomorphism is an isomorphism in any category. If $f \circ g$ and $g$ are isomorphisms, then $f$ is an isomorphism with the inverse $g \circ(f \circ g)^{-1}$. So Assumprtions 2.19 and 2.18 are satisfied. Generally, Assumption 2.20 does not hold.

Any groupoid in $\left(\mathcal{C}, \mathcal{F}_{p}\right)$ with partial covers as in Example 8.2 is a 0 -groupoid because the source and range maps are isomorphisms. Any action of a 0 -groupoid is a principal bundle by Example 5.40. Therefore, Assumptions 5.63 and 5.66 hold.

Example 8.3. Let $(\mathcal{C}, \mathcal{F})$ be a category with a subcanonical pretopology as in 47, Definition 2.1]. If $\mathcal{F}_{p}=\mathcal{F}$ then we have a category $\left(\mathcal{C}, \mathcal{F}_{p}\right)$ with partial covers.

In this case, Assumptions 2.14 and 2.15 hold by Definition in [47, Definition 2.1]. Generally, we have no additional information about the other extra assumptions. 
8.1. Category of sets. Let Sets be the category of sets.

Example 8.4. Let $\mathcal{F}_{p}$ be the collection of all maps. Then (Sets, $\mathcal{F}_{p}$ ) is a category with partial covers. The conditions (1) (2) and (3) are clearly satisfied. Also, it is clear that a map in Sets is a coequaliser if and only if it is a surjection. Therefore, the covers are the surjections.

Since the composition of surjections is a surjection, Assumption 2.14 holds. Consider a surjection $f: \mathrm{A} \rightarrow \mathrm{B}$, a map $g: \mathrm{C} \rightarrow \mathrm{B}$ and an element $c$ in $\mathrm{C}$. Since $f: \mathrm{A} \rightarrow \mathrm{B}$ is sujective, there is $a \in \mathrm{A}$ such that $f(a)=g(c)$. Thus $(a ; c) \in \mathrm{A} \times_{f, \mathrm{~B}, g} \mathrm{C}$. Since $\operatorname{pr}_{2}(a ; c)=c$, the coordinate projection $\mathrm{pr}_{2}: \mathrm{A} \times_{f, \mathrm{~B}, g} \mathrm{C} \rightarrow \mathrm{C}$ is a surjection. Therefore, Assumption 2.15 holds. Assumptions 2.19 and 2.18 are clearly satisfied. Sets does have a final object, but Assumption 2.20 does not hold. If we consider the subcategory of Sets without the empty set then any set with a single element is a final object in this subcategory, and any map to it is surjective, hence a cover. Since a fibre-product of non-empty sets along a surjective map is never empty, $\left(\mathcal{F}_{p}\right)$ still forms a stronger pretopology on the subcategory of non-empty sets, and Assumtion 2.20 is satisfied in this category.

Since Sets has arbitrary colimits, for any $G$-action $\left(X ; m_{X} ; s_{X}\right)$ there is a coequaliser $\mathrm{p}: \mathrm{X} \rightarrow \mathbf{Z}$ of the pair of maps $\mathrm{pr}_{1}, \mathrm{~m}_{\mathbf{X}}: \mathbf{X} \times_{\mathbf{s}_{\mathrm{X}}, \mathrm{G}_{0}, \mathrm{r}} \mathrm{G}^{1} \rightrightarrows \mathrm{X}$. A G-action $\left(\mathrm{X} ; \mathrm{m}_{\mathbf{X}} ; \mathrm{s}_{\mathrm{X}}\right)$ is basic if and only if the map

$$
\left(\mathrm{pr}_{1} ; \mathrm{m}_{\mathrm{X}}\right): \mathrm{X} \times_{\mathrm{s}_{\mathrm{X}}, \mathrm{G}_{0}, \mathrm{r}} \mathrm{G}^{1} \stackrel{\sim}{\rightarrow} \mathrm{X} \times_{\mathrm{p}, \mathrm{Z}, \mathrm{p}} \mathrm{X}, \quad(x ; g) \mapsto(x ; x \cdot g)
$$

is invertible. This map is surjective by construction of $\mathrm{p}: \mathrm{X} \rightarrow \mathrm{Z}$. It is injective if and only if the following condition holds: if for $x \in \mathrm{X}$ and $g, g_{1} \in \mathrm{G}^{1}$ with $\mathrm{sX}(x)=\mathrm{r}(g)=$ $\mathrm{r}\left(g_{1}\right)$ we have $x \cdot g=x \cdot g_{1}$, then $g=g_{1}$ (free action). In the case of the canonical action of $\mathrm{G}$ on its objects, the groupoid $\mathrm{G}$ is basic if and only if for any $g, g_{1} \in \mathrm{G}^{1}$ with $\mathrm{s}(g)=\mathrm{s}\left(g_{1}\right)$ and $\mathrm{r}(g)=\mathrm{r}\left(g_{1}\right)$ we have $g=g_{1}$. Consider any basic groupoid $\mathrm{G}$ and any $\mathrm{G}$-action $\left(\mathrm{X} ; \mathrm{m}_{\mathrm{X}} ; \mathrm{s} \mathrm{X}\right)$. Let $x \cdot g=x \cdot g_{1}$ for some $x \in \mathrm{X}$ and $g, g_{1} \in \mathrm{G}^{1}$ with $\mathrm{s}_{\mathbf{X}}(x)=\mathrm{r}(g)=\mathrm{r}\left(g_{1}\right)$. Then we have $\mathrm{s}(g)=\mathrm{s}_{\mathbf{X}}(x \cdot g)=\mathrm{s}_{\mathbf{X}}\left(x \cdot g_{1}\right)=\mathrm{s}\left(g_{1}\right)$. Since $\mathrm{G}$ is basic and $\mathrm{r}(g)=\mathrm{r}\left(g_{1}\right)$ and $\mathrm{s}(g)=\mathrm{s}\left(g_{1}\right)$, we have $g=g_{1}$. Therefore, any G-action $\left(\mathrm{X} ; \mathrm{m}_{\mathrm{X}} ; \mathrm{s}_{\mathrm{X}}\right)$ is basic. Hence any action of a basic groupoid is basic. So Assumptions 5.63 and 5.66 are satisfied.

8.2. Categories of topological spaces. Let Top be the category of topological spaces and continuous maps. This category is complete and cocomplete. In particular, all fibre products and all coequalisers exist.

We begin this section with a lemma which helps us to check whether Assumption 5.63 holds.

Lemma 8.5. Let $\mathrm{G}$ be a basic groupoid in the category of topological spaces (Top; $\mathcal{F}_{p}$ ) with partial covers. A G-action $\left(\mathrm{X} ; \mathrm{m}_{\mathbf{X}} ; \mathrm{s}_{\mathbf{X}}\right)$ is basic if and only if the coequaliser of the pair of continuous maps $\mathrm{pr}_{1}, \mathrm{~m}_{\mathrm{X}}: \mathrm{X} \times_{\mathrm{sX}_{\mathrm{X}}, \mathrm{G}^{0}, \mathrm{r}} \mathrm{G}^{1} \rightrightarrows \mathrm{X}$ is a cover.

Proof. If a G-action $\left(\mathrm{X} ; \mathrm{m}_{\mathrm{X}} ; \mathrm{s}_{\mathrm{X}}\right)$ is basic it is a part of a principal bundle by Definition 5.55. So we have an orbit space projection $\mathrm{p}: \mathrm{X} \rightarrow \mathrm{Z}$ which is a cover by Definition 5.33. This cover is a coequaliser of the pair of continuous maps $\mathrm{pr}_{1}, \mathrm{~m}_{\mathrm{X}}: \mathrm{X} \times_{\mathrm{sx}_{\mathrm{X}}, \mathrm{G}^{0}, \mathrm{r}} \mathrm{G}^{1} \rightrightarrows \mathrm{X}$ by Lemma 5.34

Conversely, suppose that a coequaliser $q: X \rightarrow X / G$ of the pair of continuous maps $\operatorname{pr}_{1}, m_{X}: X \times_{s_{X}, G^{0}, r} G^{1} \rightrightarrows X$ is a cover. Since the groupoid $G$ is basic, the map $(r ; s): G^{1} \rightarrow G^{0} \times_{p, Z, p} G^{0}$ is a homeomorphism by Lemma 5.59 , where $p: G^{0} \rightarrow Z$ is the orbit space projection of the canonical G-action on its objects. We have to prove that the map

$$
\left(\mathrm{pr}_{1} ; \mathrm{m}_{\mathrm{X}}\right): \mathrm{X} \times_{\mathrm{sX}_{\mathrm{X}}, \mathrm{G}_{0}, \mathrm{r}} \mathrm{G}^{1} \stackrel{\sim}{\rightarrow} \mathrm{X} \times_{\mathrm{q}, \mathrm{X} / \mathrm{G}, \mathrm{q}} \mathrm{X}, \quad(x ; g) \mapsto(x ; x \cdot g),
$$


is a homeomorphism. It is injective because if $x \cdot g=x \cdot g_{1}$, then $\mathrm{sx}_{\mathbf{X}}(x)=\mathrm{r}(g)=$ $\mathrm{r}\left(g_{1}\right)$ and $\mathrm{s}(g)=\mathrm{s}_{\mathbf{X}}(x \cdot g)=\mathrm{s}_{\mathbf{X}}\left(x \cdot g_{1}\right)=\mathrm{s}\left(g_{1}\right)$. Hence $(\mathrm{r} ; \mathrm{s})(g)=(\mathrm{r} ; \mathrm{s})\left(g_{1}\right)$. Thus $g=g_{1}$. So for any $x, x_{1} \in \mathrm{X}$ in the same G-orbit we have a unique $g \in \mathrm{G}^{1}$ with $x \cdot g=x_{1}$. This element $g$ depends continuously on $x$ and $x_{1}$ because the inverse of $(r ; s): G^{1} \rightarrow G^{0} \times_{p, Z, p} G^{0}$ is continuous. So the continuous map $\left(\mathrm{pr}_{1} ; \mathrm{m}_{\mathrm{X}}\right)$ has a continuous inverse and therefore, it is a homeomorphism.

We have different kinds of stronger pretopologies in the category of topological spaces. We begin with biquotient maps as covers. First of all, consider the main working lemma for this subsection, which is proved in [51]. We need the following definition:

Definition 8.6. A map $f: X \rightarrow Y$ is limit lifting if every convergent net in $\mathrm{Y}$ lifts to a convergent net in $\mathrm{X}$. More precisely, let $(I, \leq)$ be a directed set and let $\left(y_{i}\right)_{i \in I}$ be a net in $\mathrm{Y}$ converging to some $y \in \mathrm{Y}$. A lifting of this convergent net is a directed set $(J, \leq)$ with a surjective order-preserving map $\varphi: J \rightarrow I$ and a net $\left(x_{j}\right)_{j \in J}$ in $\mathrm{X}$ with $f\left(x_{j}\right)=y_{\varphi(j)}$ for all $j \in J$, converging to some $x \in \mathrm{X}$ with $f(x)=y$.

Definition 8.7. Let $f: X \rightarrow Y$ be a continuous surjection. It is a biquotient map if for every $y \in \mathrm{Y}$ and every open covering $\mathcal{U}$ of $f^{-1}(y)$ in $\mathrm{X}$, there are finitely many $U \in \mathcal{U}$ for which the subsets $f(U)$ cover some neighbourhood of $y$ in $Y$.

Lemma 8.8. Biquotient maps are the same as limit lifting maps.

Example 8.9. Let $\mathcal{F}_{p}$ be the collection of all maps in Top which are biquotient on its image with the subspace topology. Then (Top, $\mathcal{F}_{p}$ ) is a category with partial covers. It is clear that isomorphisms are limit lifting. Let $f: \mathrm{A} \rightarrow \mathrm{B}$ and $g: \mathrm{B} \rightarrow \mathrm{C}$ be composable maps. If $f$ and $g$ are limit lifting on their image, then so is $g \circ f$ because $\operatorname{Im}(g \circ f) \subseteq \operatorname{Im}(g)$ and since $g$ is limit lifting, any convergent net in $\operatorname{Im}(g \circ f)$ lifts to a convergent net in $\operatorname{Im}(f)$ and since $f$ is limit lifting, this convergent net lifts to a convergent net in $\mathrm{A}$. Let $\mathrm{pr}_{2}: \mathrm{A} \times_{f, \mathrm{~B}, g} \mathrm{C} \rightarrow \mathrm{C}$ be a pull-back of a limit lifting map $f: \mathrm{A} \rightarrow \mathrm{B}$ on its image along any continuous map $g: \mathrm{C} \rightarrow \mathrm{B}$. It is clear that $c \in \operatorname{Im}\left(\mathrm{pr}_{2}\right)$ if and only if $g(c) \in \operatorname{Im}(f)$. Let $\left(c_{i}\right)_{i \in I}$ be a convergent net in C. Since $g$ is continuous, $g\left(c_{i}\right)_{i \in I}$ is a convergent net in $\operatorname{Im}(f)$. This net lifts to a convergent net $\left(a_{j}\right)_{j \in J}$ in A with $f\left(a_{j}\right)=g\left(c_{\varphi(j)}\right)$ for all $j \in J$ by Definition 8.6 Therefore, we have a convergent net $\left(a_{j} ; c_{\varphi(j)}\right)$ in $\mathrm{A} \times_{f, \mathrm{~B}, g} \mathrm{C}$. Therefore, the coordinate projection $\mathrm{pr}_{2}: \mathrm{A} \times_{f, \mathrm{~B}, g} \mathrm{C} \rightarrow \mathrm{C}$ is a limit lifting map. So the property (3) holds. We know that limit lifting maps are quotient maps and quotient maps are coequalisers, so the biquotient maps on the image are coequalisers if and only if they are surjective biquotient maps. So covers are surjective biquotient maps.

In this category with such stronger pretopology, Assumptions 2.14 and 2.15 hold because the compositions of surjections is a surjection and a pull-back of a surjection is a surjection (compose in Example 8.4).

If the composition $g \circ f$ of the continuous maps $f: \mathrm{A} \rightarrow \mathrm{B}$ and $g: \mathrm{B} \rightarrow \mathrm{C}$ is limit lifting, then the map $g$ is so because any convergent net in $C$ lifts to a convergent net in $\mathrm{A}$ and then since $f: \mathrm{A} \rightarrow \mathrm{B}$ is continuous, it gives a convergent net in $\mathrm{B}$, which shows that $g$ is limit lifting. That is more then Assumption 2.19

Also, such stronger pretopology satisfies Assumption 2.20 if we remove the empty space from the category. It is clear that a space with a single element is a final object in Top, and any map from a non-empty space to it is limit lifting.

Assumption 5.63 is not satisfied. A counterexample is given in [47, Example 9.10]. We do not know whether Assumption 5.66 holds.

The following three examples are given by continuous sections. Let $f: \mathrm{A} \rightarrow \mathrm{B}$ be a continuous map in Top. We call a continuous map $\sigma_{b}: U_{b} \rightarrow \mathrm{A}$ a local continuous section for $f$ at $b \in \mathrm{B}$ if $U_{b}$ is a neighbourhood of $b$ and $f \circ \sigma_{b}=\operatorname{id}_{U_{b}}$. 
Definition 8.10. We call $f: \mathrm{A} \rightarrow \mathrm{B}$ locally split if local continuous sections $\sigma_{b}: U_{b} \rightarrow$ A exist at all $b \in \mathrm{B}$.

Example 8.11. Let $\mathcal{F}_{p}$ be the collection of all maps which are locally split onto their image. Then (Top, $\mathcal{F}_{p}$ ) is a category with partial covers. The condition (1) is clearly satisfied. Let $f: \mathrm{A} \rightarrow \mathrm{B}$ and $g: \mathrm{B} \rightarrow \mathrm{C}$ be composable maps. If $f$ and $g$ are locally split onto their image, then for any $c \in \operatorname{Im}(g \circ f) \subseteq \operatorname{Im}(g)$ we have local continuous sections $\sigma_{c}: U_{c} \rightarrow \mathrm{B}$ for $g: \mathrm{B} \rightarrow \mathrm{C}$ and $\tau_{\sigma_{c}(c)}: U_{\sigma_{c}(c)} \rightarrow \mathrm{A}$ for $f: \mathrm{A} \rightarrow \mathrm{B}$. The composition

$$
\tau_{\sigma_{c}(c)} \circ \sigma_{c}: g\left(U_{\sigma_{c}(c)} \cap \sigma_{c}\left(U_{c}\right)\right) \rightarrow \mathrm{A}
$$

is a local section for $g \circ f$. Hence the condition (2) holds. Let $\mathrm{pr}_{2}: \mathrm{A} \times_{f, \mathrm{~B}, g} \mathrm{C} \rightarrow \mathrm{C}$ be a pull-back of a map $f: A \rightarrow B$ that is locally split onto its image along any continuous map $g: \mathrm{C} \rightarrow \mathrm{B}$. For any $c \in \operatorname{Im}\left(\mathrm{pr}_{2}\right)$ we have a local continuous section $\sigma_{g(c)}: U_{g(c)} \rightarrow$ A for $f$. Since $U_{g(c)} \subseteq \operatorname{Im}(f)$, we have $g^{-1}\left(U_{g(c)}\right) \subseteq \operatorname{Im}\left(\mathrm{pr}_{2}\right)$. So we have a local section $\sigma_{g(c)} \circ g: g^{-1}\left(U_{g(c)}\right) \rightarrow \mathrm{A} \times_{f, \mathrm{~B}, g} \mathrm{C}$ for $\mathrm{pr}_{2}: \mathrm{A} \times_{f, \mathrm{~B}, g} \mathrm{C} \rightarrow \mathrm{C}$. Therefore, the condition (3) holds. Any locally split map is a biquotient map because any convergent net can be lifted by a local continuous section. A map biquotient on its image is a coequaliser if and only if it is surjective. Therefore, a locally split map onto its image is a coequaliser if and only if it is surjective. So the covers are the locally split surjections.

As in the previous case, Asssumptions 2.14 and 2.15 hold for the same reason: The composition of surjections is a surjection and a pull-back of a surjection is a surjection.

If the composition $g \circ f$ of the continuous maps $f: \mathrm{A} \rightarrow \mathrm{B}$ and $g: \mathrm{B} \rightarrow \mathrm{C}$ is a locally split map, then the map $g$ is so because for any $c \in C$ we have a local continuous section $\sigma_{c}: U_{c} \rightarrow \mathrm{A}$ for $g \circ f$ and this gives a local continuous section $f \circ \sigma_{c}: U_{c} \rightarrow \mathrm{B}$ for $g: \mathrm{B} \rightarrow \mathrm{C}$. That is more than Assumption 2.19, Therefore, Assumption 2.18 holds, too.

It is also clear that the constant map $f: \mathrm{A} \rightarrow\{*\}$ from any non-empty space A to the one-point space $\{*\}$ has a continuous section $\sigma_{*}:\{*\} \rightarrow A$, which gives Assumption 2.20 if we exclude the empty space.

Lemma 8.12. Let $\mathrm{G}$ be any basic groupoid in (Top, $\mathcal{F}_{p}$ ) defined in Example 8.11, and let $\left(\mathrm{X} ; \mathrm{m}_{\mathrm{X}} ; \mathrm{s}_{\mathrm{X}}\right)$ be any $\mathrm{G}$-action. A coequaliser $\mathrm{q}: \mathrm{X} \rightarrow \mathrm{X} / \mathrm{G}$ of the pair of continuous maps $\mathrm{pr}_{1}, \mathrm{~m}_{\mathrm{X}}: \mathrm{X} \times_{\mathrm{s}_{\mathrm{X}}, \mathrm{G}^{0}, \mathrm{r}} \mathrm{G}^{1} \rightrightarrows \mathrm{X}$ is locally split.

Proof. We have that $\mathrm{G}$ is basic. That is, there is a locally split continuous map $\mathrm{p}: \mathrm{G}^{0} \rightarrow Z$ such that $(\mathrm{r} ; \mathrm{s}): \mathrm{G}^{1} \rightarrow \mathrm{G}^{0} \times_{\mathrm{p}, \mathrm{Z}, \mathrm{p}} \mathrm{G}^{0}$ is a well-defined homeomorphism. Let $\left(X ; m_{X} ; s_{X}\right)$ be any $G$-action. We are going to show that a coequaliser $q: X \rightarrow X / G$ of the pair of continuous maps $\mathrm{pr}_{1}, \mathrm{~m}_{\mathrm{X}}: \mathrm{X} \times_{\mathrm{sX}_{\mathrm{X}}, \mathrm{G}^{0}, \mathrm{r}} \mathrm{G}^{1} \rightrightarrows \mathrm{X}$ is locally split. Since $\mathrm{p}\left(\mathrm{s}_{\mathbf{X}}(x \cdot g)\right)=\mathrm{p}(\mathrm{s}(g))=\mathrm{p}(\mathrm{r}(g))=\mathrm{p}\left(\mathrm{s}_{\mathbf{X}}(x)\right)$, for all $x \in \mathrm{X}, g \in \mathrm{G}^{1}$ with $\mathrm{sX}_{\mathbf{X}}(x)=\mathrm{r}(g)$, there is a well-defined continuous map $\tilde{\mathbf{s}}_{\mathbf{X}}: \mathbf{X} / \mathbf{G} \rightarrow \mathbf{Z}$ with $\tilde{\mathbf{s}}_{\mathbf{X}}([x])=\left[\mathbf{s}_{\mathbf{X}}(x)\right]$, for all $x \in \mathrm{X}$. For any $\left[x_{0}\right] \in \mathrm{X} / \mathrm{G}$ we have a neighbourhood $U_{\left[s_{\mathbf{X}}\left(x_{0}\right)\right]}$ of $\left[s_{\mathbf{X}}\left(x_{0}\right)\right] \in \mathbf{Z}$ and a local continuous section $\sigma_{\left[\mathrm{s}_{\mathrm{x}}\left(x_{0}\right)\right]}: U_{\left[\mathrm{sx}_{\mathrm{x}}\left(x_{0}\right)\right]} \rightarrow \mathrm{G}^{0}$ for $\mathrm{p}: \mathrm{G}^{0} \rightarrow \mathrm{Z}$. We can construct a local continuous section for $\mathbf{q}: X \rightarrow X / G$ defined on $\left(\tilde{s}_{X}\right)^{-1}\left(U_{\left[\mathrm{sx}_{X}\left(x_{0}\right)\right]}\right) \subseteq X / G$. Let $[x] \in\left(\tilde{s}_{\mathbf{X}}\right)^{-1}\left(U_{\left[\mathbf{s X}_{X}\left(x_{0}\right)\right]}\right)$. We have an element $\left(\mathrm{s}_{\mathbf{X}}(x) ; \sigma_{\left[\mathbf{s X}_{X}\left(x_{0}\right)\right]}\left(\left[\mathbf{s}_{\mathbf{X}}(x)\right]\right)\right)$ in $\mathrm{G}^{0} \times_{\mathrm{p}, \mathrm{Z}, \mathrm{p}} \mathrm{G}^{0}$.

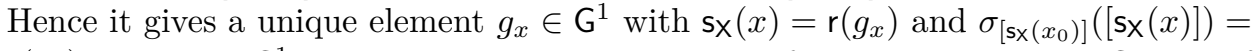
$\mathrm{s}\left(g_{x}\right)$. Let $g_{x_{1}} \in \mathrm{G}^{1}$ be constructed in the same way for another $x_{1}$ in the G-orbit of 
$x$. We have

$$
\begin{aligned}
\mathrm{s}_{\mathbf{X}}\left(x \cdot g_{x}\right) & =\mathrm{s}\left(g_{x}\right) \\
& =\sigma_{\left[\mathrm{sX}_{\mathbf{X}}\left(x_{0}\right)\right]}\left(\left[\mathrm{sx}_{\mathbf{X}}(x)\right]\right) \\
& =\sigma_{\left[\mathbf{s}\left(x_{0}\right)\right]}\left(\left[\mathrm{sX}_{\mathbf{X}}\left(x_{1}\right)\right]\right) \\
& =\mathrm{s}\left(g_{x_{1}}\right) \\
& =\mathrm{sx}_{\mathbf{X}}\left(x_{1} \cdot g_{x_{1}}\right) .
\end{aligned}
$$

Since $x$ and $x_{1}$ are in the same G-orbit, there is $g \in \mathrm{G}^{1}$ with $x \cdot g=x_{1}$. Thus

$$
\begin{aligned}
\mathrm{s}\left(g_{x}\right) & =\mathrm{s}_{\mathbf{X}}\left(x \cdot g_{x}\right) \\
& =\mathrm{s}_{\mathbf{X}}\left(x_{1} \cdot g_{x_{1}}\right) \\
& =\mathrm{s}_{\mathbf{X}}\left(x \cdot g \cdot g_{x_{1}}\right) \\
& =\mathrm{s}\left(g \cdot g_{x_{1}}\right) .
\end{aligned}
$$

We also have $\mathrm{r}\left(g_{x}\right)=\mathrm{s}_{\mathbf{X}}(x)=\mathrm{r}(g)=\mathrm{r}\left(g \cdot g_{x_{1}}\right)$. Thus $g_{x}=g \cdot g_{x_{1}}$ because $\mathrm{G}$ is basic. Hence $x \cdot g_{x}=x \cdot g \cdot g_{x_{1}}=x_{1} \cdot g_{x_{1}}$. Therefore, we have a well-defined continuous map defined on $\left(\tilde{\mathbf{s}}_{\mathbf{X}}\right)^{-1}\left(U_{\left[\mathrm{sx}\left(x_{0}\right)\right]}\right) \subseteq \mathrm{X} / \mathrm{G}$ by $[x] \mapsto x \cdot g_{x}$. This clearly gives a local continuous section for $\mathrm{q}$.

Assumptions 5.63 and 5.66 hold by Lemma 8.12 and Lemma 8.5 .

As in the previous case, the next example is defined using continuous sections. Let $f: \mathrm{A} \rightarrow \mathrm{B}$ be a continuous map.

Definition 8.13. $f: \mathrm{A} \rightarrow \mathrm{B}$ has many local continuous sections if for all $a \in \mathrm{A}$ there is an open neighbourhood $U_{a} \subseteq \mathrm{B}$ of $f(a)$ and a continuous map $\sigma_{a}: U_{a} \rightarrow \mathrm{A}$ with $\sigma_{a}(f(a))=a$ and $f \circ \sigma_{a}=\operatorname{id}_{U_{a}}$.

Example 8.14. Let $\mathcal{F}_{p}$ be the collection of all continuous maps with many local continuous sections. Then (Top, $\mathcal{F}_{p}$ ) is a category with partial covers. The condition (1) is clearly satisfied. Let $f: \mathrm{A} \rightarrow \mathrm{B}$ and $g: \mathrm{B} \rightarrow \mathrm{C}$ be composable maps with many local continuous sections. For any $a \in \mathrm{A}$ we have the neighbourhoods $U_{a} \subseteq \mathrm{B}$ of $f(a) \in \mathrm{B}$ and $V_{f(a)} \in \mathrm{C}$ of $g(f(a)) \in \mathrm{C}$ and the local continuous sections $\sigma_{a}: U_{a} \rightarrow \mathrm{A}$ for $f: \mathrm{A} \rightarrow \mathrm{B}$ with $\sigma_{a}(f(a))=a$ and $\tau_{f(a)}: V_{f(a)} \rightarrow \mathrm{B}$ for $g: \mathrm{B} \rightarrow \mathrm{A}$ with $\tau_{f(a)}(g(f(a)))=f(a)$. The composition

$$
\sigma_{a} \circ \tau_{f(a)}: g\left(U_{a} \cap \tau_{f(a)}\left(V_{f(a)}\right)\right) \rightarrow \mathrm{A}
$$

is a local section for $g \circ f$ with $\left(\sigma_{a} \circ \tau_{f(a)}\right)(g(f(a)))=a$. Hence the condition (2) holds. Let $\mathrm{pr}_{2}: \mathrm{A} \times_{f, \mathrm{~B}, g} \mathrm{C} \rightarrow \mathrm{C}$ be a pull-back of a continuous map $f: \mathrm{A} \rightarrow \mathrm{B}$ with many local continuous sections along any continuous map $g: \mathrm{C} \rightarrow \mathrm{B}$. For any $(a ; c) \in \mathrm{A} \times_{f, \mathrm{~B}, g} \mathrm{C}$ we have a neighbourhood $U_{a} \subseteq \mathrm{B}$ of $f(a)=g(c) \in \mathrm{B}$ and a local continuous section $\sigma_{a}: U_{a} \rightarrow$ A for $f$ with $\sigma_{a}(f(a))=a$. There is a neighbourhood $g^{-1}\left(U_{a}\right) \subseteq \mathrm{C}$ of $c$ and a local continuous section $\left(\sigma_{a} \circ g ; \mathrm{id}_{\mathrm{C}}\right): g^{-1}\left(U_{a}\right) \rightarrow \mathrm{A} \times_{f, \mathrm{~B}, g} \mathrm{C}$ for $\mathrm{pr}_{2}: \mathrm{A} \times_{f, \mathrm{~B}, g} \mathrm{C} \rightarrow \mathrm{C}$ with $\left(\sigma_{a} \circ g ; \operatorname{id}_{\mathrm{C}}\right)(c)=(a ; c)$. Therefore, the condition $(3)$ holds. Any continuous map with many local continuous sections is a biquotient map on its image because any convergent net in the image can be lifted by a local continuous section. A biquotient map on its image is a coequaliser if and only if it is surjective. Therefore, a continuous map with many local continuous sections is a coequaliser if and only if it is surjective. So the covers are the surjections with many local continuous sections

As in the case of the biquotient maps, Asssumptions 2.14 and 2.15 hold. The composition of surjections is a surjection and a pull-back of a surjection is a surjection.

Let $f: \mathrm{A} \rightarrow \mathrm{B}$ and $g: \mathrm{B} \rightarrow \mathrm{C}$ be composable continuous maps. Assume that the composition $g \circ f$ and $f$ are surjections and that they have many local continuous 
sections. For any $b \in \mathrm{B}$ we have an element $a \in \mathrm{A}$ with $f(a)=b$. We also have a neighbourhood $U_{a} \subseteq \mathrm{C}$ of $g(b)$ and a local continuous section $\sigma_{a}: U_{a} \rightarrow$ A for $g \circ f$ with $\sigma_{a}(g(b))=a$. There is a local continuous section $f \circ \sigma_{a}: U_{a} \rightarrow \mathrm{B}$ for $g: \mathrm{B} \rightarrow \mathrm{C}$ with $\left(f \circ \sigma_{a}\right)(g(b))=b$. Therefore, Assumptions 2.19 and 2.18 hold.

It is also clear that the constant map $f: A \rightarrow\{*\}$ from any non-empty space $A$ to the one-point space $\{*\}$ has a continuous section for any point in $A$, which gives Assumption 2.20 if we exclude the empty space from the category.

Suppose we have all data from Lemma 8.12 . For any $x \in \mathrm{X}$ we have a neighbourhood $U_{\mathrm{sX}_{\mathrm{X}}(x)} \subseteq \mathrm{Z}$ of $\left[\mathrm{sX}_{\mathrm{X}}(x)\right]$ and a local continuous section $\sigma_{\mathrm{sX}_{\mathrm{X}}(x)}: U_{\mathrm{sX}_{\mathrm{X}}(x)} \rightarrow \mathrm{G}^{0}$ for $\mathrm{p}: \mathrm{G}^{0} \rightarrow \mathrm{Z}$ with $\sigma_{\mathrm{sX}_{\mathrm{X}}(x)}\left(\left[\mathrm{s}_{\mathrm{X}}(x)\right]\right)=\mathrm{s}_{\mathrm{X}}(x)$. Then we construct a local continuous section for $\mathrm{q}: \mathrm{X} \rightarrow \mathrm{X} / \mathrm{G}$ defeined on $\left(\tilde{\mathrm{s}}_{\mathrm{X}}\right)^{-1}\left(U_{\left[\mathrm{sX}_{\mathbf{X}}(x)\right]}\right) \subseteq \mathrm{X} / \mathrm{G}$ by $[x] \mapsto x \cdot g_{x}$. In this case $g_{x}=1_{\mathrm{sX}(x)}$. Therefore, the map $\mathrm{q}: \mathrm{X} \rightarrow \mathrm{X} / \mathrm{G}$ has many local continuous sections. So Assumptions 5.63 and 5.66 hold by Lemma 8.5

The next example is defined by global continuous sections. Let $f: \mathrm{A} \rightarrow \mathrm{B}$ be a continuous map.

Definition 8.15. $f: \mathrm{A} \rightarrow \mathrm{B}$ is a splitting map if there is a continuous section $\sigma_{f}: \mathrm{B} \rightarrow \mathrm{A}$ with $f \circ \sigma_{f}=\mathrm{id}_{\mathrm{B}}$.

Example 8.16. Let $\mathcal{F}_{p}$ be the collection of all continuous maps in Top which split on the image. Then (Top, $\left.\mathcal{F}_{p}\right)$ is a category with partial covers. The conditions (1) is clearly satisfied. Let $f: \mathrm{A} \rightarrow \mathrm{B}$ and $g: \mathrm{B} \rightarrow \mathrm{C}$ be composable splitting maps on the image. Since $\operatorname{Im}(g \circ f) \subseteq \operatorname{Im}(g)$, we have a split $\left.\sigma_{f} \circ \sigma_{g}\right|_{\operatorname{Im}(g \circ f)}: \operatorname{Im}(g \circ f) \rightarrow \mathrm{A}$ for $g \circ f$, where $\left.\sigma_{g}\right|_{\operatorname{Im}(g \circ f)}$ is a restriction of $\sigma_{g}$ on $\operatorname{Im}(g \circ f)$. So the condition (2) holds. Let $\mathrm{pr}_{2}: \mathrm{A} \times_{f, \mathrm{~B}, g} \mathrm{C} \rightarrow \mathrm{C}$ be a pull-back of a splitting map $f: \mathrm{A} \rightarrow \mathrm{B}$ on its image along any continuous map $g: \mathrm{C} \rightarrow \mathrm{B}$. For any $c \in \operatorname{Im}\left(\mathrm{pr}_{2}\right)$ we have that $g(c) \in \operatorname{Im}(f)$. Therefore, we have a split $\left(\sigma_{f} \circ g ; \operatorname{id}_{\mathrm{C}}\right): \operatorname{Im}\left(\mathrm{pr}_{2}\right) \rightarrow \mathrm{A} \times{ }_{f, \mathrm{~B}, g} \mathrm{C}$ for $\mathrm{pr}_{2}: \mathrm{A} \times_{f, \mathrm{~B}, g} \mathrm{C} \rightarrow \mathrm{C}$ defined on the image of $\mathrm{pr}_{2}$. So the condition (3) holds. Any splitting map is a biquotient map because any convergent net can be lifted by a continuous section. A biquotient map on its image is a coequaliser if and only if it is surjective. Therefore, a splitting map on its image is a coequaliser if and only if it is surjective. So the covers are the splitting surjections.

Asssumptions 2.14 and 2.15 hold because the composition of surjections is a surjection and a pull-back of a surjection is a surjection.

If the composition $g \circ f$ of the continuous maps $f: \mathrm{A} \rightarrow \mathrm{B}$ and $g: \mathrm{B} \rightarrow \mathrm{C}$ is a splitting map, then the map $g$ is so because we have a continuous section $f \circ \sigma_{f \circ g}: \mathrm{C} \rightarrow \mathrm{B}$ for $g: \mathrm{B} \rightarrow \mathrm{C}$. That is more than Assumption 2.19 Therefore, Assumption 2.18 holds, too.

The constant map $f: \mathrm{A} \rightarrow\{*\}$ from any non-empty space $\mathrm{A}$ to the one-point space $\{*\}$ has a continuous section $\sigma:\{*\} \rightarrow$ A, where $\sigma(*)$ is any point in A. This gives Assumption 2.20 if we exclude the empty space.

Suppose we have all data from Lemma 8.12 In this case $U_{\left[\mathbf{s}_{\mathbf{X}}\left(x_{0}\right)\right]}=\mathrm{Z}$. Also $\left(\tilde{\mathbf{s}}_{X}\right)^{-1}(Z)=X / G$. Therefore, the constructed local section is global in this case. So Assumptions 5.63 and 5.66 hold by Lemma 8.5

The next example is given by using proper maps. Let $f: \mathrm{A} \rightarrow \mathrm{B}$ be a continuous map. $f$ is closed if it maps closed subsets to closed subsets. It is proper if and only if it is closed and $f^{-1}(b)$ is quasi-compact for all $b \in \mathrm{B}$. ([4, I.10.2]).

Lemma 8.17 ([4, I.10.1]). A continuous map $f: \mathrm{A} \rightarrow \mathrm{B}$ is proper if and only if the map $f \times \mathrm{id}_{\mathbf{X}}: \mathrm{A} \times \mathrm{X} \rightarrow \mathrm{B} \times \mathbf{X}$ is closed for any topological space $\mathbf{X}$.

Example 8.18. Let $\mathcal{F}_{p}$ be the collection of all proper maps in Top. Then (Top, $\left.\mathcal{F}_{p}\right)$ is a category with partial covers. The condition (1) is clearly satisfied. Let $f: A \rightarrow B$ and $g: \mathrm{B} \rightarrow \mathrm{C}$ be composable proper maps. For any topological space $\mathrm{X}$, the maps 
$f \times \mathrm{id}_{\mathrm{X}}: \mathrm{A} \times \mathrm{X} \rightarrow \mathrm{B} \times \mathrm{X}$ and $g \times \mathrm{id}_{\mathrm{X}}: \mathrm{B} \times \mathrm{X} \rightarrow \mathrm{C} \times \mathrm{X}$ are closed. It is clear that the composition of closed maps is closed. Hence $(g \circ f) \times \mathrm{id} \times \mathrm{A} \times \mathrm{X} \rightarrow \mathrm{C} \times \mathrm{X}$ is closed for any topological space $\mathbf{X}$. Thus $g \circ f$ is proper. So the condition (2) is satisfied. Let $\mathrm{pr}_{2}: \mathrm{A} \times_{f, \mathrm{~B}, g} \mathrm{C} \rightarrow \mathrm{C}$ be the pull-back of a proper map $f: \mathrm{A} \rightarrow \mathrm{B}$ along any continuous map $g: C \rightarrow B$. Since $f: A \rightarrow \operatorname{Im}(f) \subseteq B$ is proper, the map $f \times \operatorname{id}_{\mathrm{C}} \times \mathrm{id}_{\mathrm{X}}: \mathrm{A} \times g^{-1}(\operatorname{Im}(f)) \times \mathrm{X} \rightarrow \operatorname{Im}(f) \times g^{-1}(\operatorname{Im}(f)) \times \mathbf{X}$ is closed for any topological space $X$ by Lemma 8.17. Consider

$$
g^{-1}(\operatorname{Im}(f)) \times \mathrm{X} \cong\left\{(g(c) ; c ; x) \mid \forall c \in g^{-1}(\operatorname{Im}(f)), \forall x \in \mathrm{X}\right\},
$$

a subset of $\operatorname{Im}(f) \times g^{-1}(\operatorname{Im}(f)) \times \mathbf{X}$. It is clear that the restriction of the map $f \times \operatorname{id}_{\mathrm{C}} \times \mathrm{id}_{\mathrm{X}}$ on the subset $\left(f \times \mathrm{id}_{\mathrm{C}} \times \mathrm{id}_{\mathrm{X}}\right)^{-1}\left(g^{-1}(\operatorname{Im}(f)) \times \mathrm{X}\right)$ is closed, too. This gives that the map $\mathrm{pr}_{2} \times \mathrm{id} \mathrm{X}:\left(\mathrm{A} \times_{f, \mathrm{~B}, g} \mathrm{C}\right) \times \mathrm{X} \rightarrow \operatorname{Im}\left(\mathrm{pr}_{2}\right) \times \mathrm{X}$ is closed for any topological space $\mathbf{X}$ because $\operatorname{Im}\left(\mathrm{pr}_{2}\right)=g^{-1}(\operatorname{Im}(f))$. Therefore, $\mathrm{pr}_{2}$ is proper. So the condition (3) is satisfied. Proper maps are biquotient maps on the image by [32, Proposition 3.2]. Hence the proper maps are coequalisers if and only if they are proper surjections. Therefore, the covers are the surjective proper maps.

Asssumptions 2.14 and 2.15 hold because the composition of surjections is a surjection and a pull-back of a surjection is a surjection.

Let $f: \mathrm{A} \rightarrow \mathrm{B}$ and $g: \mathrm{B} \rightarrow \mathrm{C}$ be composable continuous maps. If $g \circ f$ and $f$ are surjective proper maps, then the map $g$ is so. For any topological space $X$ the maps $(g \circ f) \times \operatorname{id}_{\mathbf{X}}: \mathrm{A} \times \mathrm{X} \rightarrow \mathrm{C} \times \mathbf{X}$ and $f \times \mathrm{id}_{\mathbf{X}}: \mathrm{A} \times \mathbf{X} \rightarrow \mathrm{B} \times \mathbf{X}$ are closed. Let $U$ be any closed subset in $B \times X$. Since $f \times \operatorname{idx}$ is a continuous, the subset $\left(f \times \mathrm{id}_{\mathbf{X}}\right)^{-1}(U)$ is closed in $\mathrm{A} \times \mathrm{X}$. Since $g \circ f$ is proper, $((g \circ f) \times \mathrm{idx})\left(\left(f \times \mathrm{id}_{\mathbf{X}}\right)^{-1}(U)\right)$ is closed in $\mathrm{C} \times \mathrm{X}$. This closed subset equals $\left(g \times \mathrm{id}_{\mathrm{X}}\right)(U)$ because $f \times \mathrm{idx}$ is surjective. Therefore, Assumptions 2.19 and 2.18 are satisfied by Lemma 8.17

The map from a space $A$ to the one-point space is proper if and only if $A$ is quasi-compact. Hence Assumption 2.20 fails even if we exclude the empty space.

We have no information about Assumptions 5.63 and 5.66 .

The next example is defined using open maps. Let $f: \mathrm{A} \rightarrow \mathrm{B}$ be a continuous map.

Definition 8.19. $f$ is open if the image of all open subsets of $A$ is open in $B$.

Lemma 8.20 ( 51 , Proposition 1.15]). A continuous surjection $f: \mathrm{A} \rightarrow \mathrm{B}$ between topological spaces is open if and only if, for any $a \in \mathrm{A}$, a convergent net $\left(b_{i}\right)_{i \in I}$ in $\mathrm{B}$ with $\lim _{i \in I} b_{i}=f(a)$ lifts to a net in $\mathrm{A}$ converging to a.

Example 8.21. Let $\mathcal{F}_{p}$ be the collection of all open maps in Top. Then (Top, $\mathcal{F}_{p}$ ) is a category with partial covers. The condition (1) is clearly satisfied. Let $f: A \rightarrow B$ and $g: \mathrm{B} \rightarrow \mathrm{C}$ be composable open maps and let $U$ be open in A. Since $f: \mathrm{A} \rightarrow-\rightarrow \mathrm{B}$ is open, $f(U)$ is open in B. Since $g: \mathbf{B} \rightarrow$ C is open, $g(f(U))$ is open in C. Therefore, $g \circ f$ is open. So the condition (2) is satisfied. Let $\mathrm{pr}_{2}: \mathrm{A} \times{ }_{f, \mathrm{~B}, g} \mathrm{C} \rightarrow \mathrm{C}$ be a pull-back of an open map $f: \mathrm{A} \rightarrow \mathrm{B}$ along a continuous map $g: \mathrm{C} \rightarrow \mathrm{B}$. Consider any element $(a ; c)$ in $\mathrm{A} \times_{f, \mathrm{~B}, g} \mathrm{C}$ and any convergent net $\left(c_{i}\right)_{i \in I}$ in $\operatorname{Im}\left(\mathrm{pr}_{2}\right)=g^{-1}(f(\mathrm{~A}))$ such that $\lim _{i \in I} c_{i}=c$. Since $g: \mathrm{B} \rightarrow \mathrm{C}$ is continuous, the net $g\left(c_{i}\right)_{i \in I}$ converges to $g(c)$. Since $f(a)=g(c)$ and $f: \mathrm{A} \rightarrow \mathrm{B}$ is open, there is a net $\left(a_{j}\right)_{j \in J}$ in A with $f\left(a_{j}\right)=g\left(c_{\varphi(j)}\right)$ for all $j \in J$, converging to $a$. Hence we have a net $\left(a_{j} ; c_{\varphi(j)}\right)_{j \in J}$

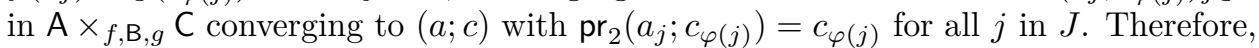
the coordinate projection $\mathrm{pr}_{2}: \mathrm{A} \times_{f, \mathrm{~B}, g} \mathrm{C} \rightarrow \mathrm{C}$ is open. So the property (3) holds. An open map is a limit lifting map on the image by Lemma 8.20 Hence the open surjections are biquotient maps, and therefore, an open map is a coequaliser if and only if it is surjective. So the covers are the open surjections.

Asssumptions 2.14 and 2.15 hold because the composition of surjections is a surjection and a pull-back of a surjection is a surjection. 
If the composition $g \circ f$ of an open surjection $f: \mathrm{A} \rightarrow \mathrm{B}$ and a continuous map $g: \mathrm{B} \rightarrow \mathrm{C}$ is an open surjection, then the map $g$ is an open surjection because for any open subset $U$ of $\mathrm{B}$ we have an open subset $f^{-1}(U)$ in A and since $g \circ f$ is open and $f$ is surjective, the subset $g\left(f\left(f^{-1}(U)\right)\right)=g(U)$ is open in C. It is clear that $g$ is surjective. So Assumptions 2.19 and 2.18 hold.

It is clear that any map from a non-empty space to a space with a single element is an open surjection. So Assumption 2.20 is satisfied if we remove the empty space from the category.

Lemma 8.22. Let $\mathrm{G}$ be a groupoid and let $\left(\mathrm{X} ; \mathrm{m}_{\mathrm{X}} ; \mathrm{s}_{\mathrm{X}}\right)$ be a $\mathrm{G}$-action. A coequaliser $\mathrm{q}: \mathrm{X} \rightarrow \mathrm{X} / \mathrm{G}$ of the pair of continuous maps $\mathrm{pr}_{1}, \mathrm{~m}_{\mathrm{X}}: \mathrm{X} \times_{\mathrm{sX}_{\mathrm{X}}, \mathrm{G}^{0}, \mathrm{r}} \mathrm{G}^{1} \rightrightarrows \mathrm{X}$ is open.

Proof. Let $\mathrm{G}$ be any groupoid, not necessary basic, and let $\left(\mathrm{X} ; \mathrm{m}_{\mathrm{X}} ; \mathrm{s}_{\mathrm{X}}\right)$ be a $\mathrm{G}$ action. We are going to prove that a coequaliser $q: X \rightarrow X / G$ of the pair of continuous maps $\mathrm{pr}_{1}, \mathrm{~m}_{\mathrm{X}}: \mathrm{X} \times_{\mathrm{sX}_{\mathrm{X}}, \mathrm{G}^{0}, \mathrm{r}} \mathrm{G}^{1} \rightrightarrows \mathrm{X}$ is open. Let $U \subseteq \mathrm{X}$ be open. Then $\mathrm{q}^{-1}(\mathrm{q}(U))=\left\{x \cdot g \mid x \in U, g \in \mathrm{G}^{1} ; \mathrm{s}_{\mathrm{X}}(x)=\mathrm{r}(g)\right\}$. This is $\mathrm{m}\left(\left(U \times \mathrm{G}^{1}\right) \cap\left(\mathrm{X} \times \mathrm{s}_{\mathrm{s}, \mathrm{G}^{0}, \mathrm{r}} \mathrm{G}^{1}\right)\right)$, which is open because $\left(U \times G^{1}\right) \cap\left(X \times_{s, G^{0}, r} G^{1}\right)$ is open in $\left(X \times_{s, G^{0}, r} G^{1}\right)$ and $m$ is an open surjection by Lemma 5.6. Thus $\mathrm{q}(U)$ is open in $\mathrm{X} / \mathrm{G}$, and $\mathrm{q}$ is open.

Any action of a basic groupoid is basic by Lemmas 8.22 and 8.5 . So Assumptions 5.63 and 5.66 hold.

The next example is defined by using the étale maps. Let $f: \mathrm{A} \rightarrow \mathrm{B}$ be a continuous map.

Definition 8.23. $f: \mathrm{A} \rightarrow \mathrm{B}$ is étale if for all $a \in \mathrm{A}$ there is an open neighbourhood $U_{a}$ such that $f\left(U_{a}\right)$ is open and $\left.f\right|_{U_{a}}: U_{a} \stackrel{\sim}{\rightarrow} f\left(U_{a}\right)$ is a homeomorphism for the subspace topologies on $U_{a}$ and $f\left(U_{a}\right)$ from $\mathrm{A}$ and $\mathrm{B}$, respectively.

Example 8.24. Let $\mathcal{F}_{p}$ be the collection of all étale maps in Top. Then (Top, $\left.\mathcal{F}_{p}\right)$ is a category with partial covers. The condition (1) is clearly satisfied. Let $f: A \rightarrow B$ and $g: \mathrm{B} \rightarrow \mathrm{C}$ be composable étale maps and let $a$ be any element in $\mathrm{A}$. Since $f: \mathrm{A} \rightarrow \mathrm{B}$ is étale, we have an open neighbourhood $U_{a}$ of $a$ and a homeomorphism $\left.f\right|_{U_{a}}: U_{a} \stackrel{\sim}{\rightarrow} f\left(U_{a}\right)$. Since $g: \mathrm{A} \rightarrow \mathrm{B}$ is étale, we have an open neighbourhood $V_{f(a)}$ of $f(a)$ and a homeomorphism $\left.g\right|_{V_{f(a)}}: V_{f(a)} \stackrel{\sim}{\rightarrow} g\left(V_{f(a)}\right)$. The subset $f\left(U_{a}\right) \cap V_{f(a)}$ is open in $f\left(U_{a}\right)$ and $f(a) \in f\left(U_{a}\right) \cap V_{f(a)}$. So $f^{-1}\left(f\left(U_{a}\right) \cap V_{f(a)}\right)$ is an open neighbourhood of $a$. The map

$$
\left.(g \circ f)\right|_{f^{-1}\left(f\left(U_{a}\right) \cap V_{f(a)}\right)}: f^{-1}\left(f\left(U_{a}\right) \cap V_{f(a)}\right) \rightarrow g\left(f\left(U_{a}\right) \cap V_{f(a)}\right)
$$

is a homeomorphism as a composition of homeomorphisms. Therefore, $g \circ f$ is étale. So the condition (2) is satisfied. Let $\mathrm{pr}_{2}: \mathrm{A} \times_{f, \mathrm{~B}, g} \mathrm{C} \rightarrow \mathrm{C}$ be a pull-back of an open map $f: \mathrm{A} \rightarrow \mathrm{B}$ along a continuous map $g: \mathrm{C} \rightarrow \mathrm{B}$. Consider any element $(a ; c)$ in $\mathrm{A} \times{ }_{f, \mathrm{~B}, g} \mathrm{C}$. Since $f: \mathrm{A} \rightarrow \mathrm{B}$ is étale, we have an open neighbourhood $U_{a}$ of $a$ and a homeomorphism $\left.f\right|_{U_{a}}: U_{a} \stackrel{\sim}{\rightarrow} f\left(U_{a}\right)$. Since $g: \mathrm{A} \rightarrow \mathrm{B}$ is continuous, the subset $g^{-1}\left(f\left(U_{a}\right)\right)$ is open in $\mathrm{C}$. The element $(a ; c)$ belongs to the subset

$$
U_{a} \times_{\left.f\right|_{U_{a}}, f\left(U_{a}\right),\left.g\right|_{g^{-1}\left(f\left(U_{a}\right)\right)}} g^{-1}\left(f\left(U_{a}\right)\right)
$$

of $\mathrm{A} \times{ }_{f, \mathrm{~B}, g} \mathrm{C}$, which is open, and the restriction of $\mathrm{pr}_{2}: \mathrm{A} \times_{f, \mathrm{~B}, g} \mathrm{C} \rightarrow \mathrm{C}$ to it is a homeomorphism because the pull-back of the homeomorphism $\left.f\right|_{U_{a}}: U_{a} \stackrel{\sim}{\rightarrow} f\left(U_{a}\right)$ along $\left.g\right|_{g^{-1}\left(f\left(U_{a}\right)\right)}$ is a homeomorphism. Therefore, $\mathrm{pr}_{2}: \mathrm{A} \times_{f, \mathrm{~B}, g} \mathrm{C} \rightarrow \mathrm{C}$ is étale. So the condition (3) holds. It is clear that étale surjections are limit liftting, and therefore, they are biquotient maps. Hence étale maps are coequalisers if and only if they are étale surjections. Therefore, the covers are the étale surjections.

Asssumptions 2.14 and 2.15 hold because the composition of surjections is a surjection and a pull-back of a surjection is a surjection. 
Let the composition $g \circ f$ of an étale surjection $f: A \rightarrow B$ and a continuous map $g: \mathrm{B} \rightarrow \mathrm{C}$ be an étale surjection. For any element $b$ in $\mathrm{B}$ we have an element $a$ in $\mathrm{A}$ and an open neighbourhood $U_{a}$ of $a$ such that $f(a)=b$ and the restriction $\left.f\right|_{U_{a}}: U_{a} \stackrel{\sim}{\rightarrow} f\left(U_{a}\right)$ is a homeomorphism. Since $g \circ f$ is étale, we have an open neighbourhood $V_{a}$ of $a$ such that the restriction $\left.(g \circ f)\right|_{V_{a}}: V_{a} \stackrel{\sim}{\rightarrow} g\left(f\left(U_{a}\right)\right)$ is a homeomorphism. The map

$$
\left.\left.(g \circ f)\right|_{U_{a} \cap V_{a}} \circ\left(\left.f\right|_{U_{a}}\right)^{-1}\right|_{f\left(U_{a}\right) \cap f\left(V_{a}\right)}: f\left(U_{a}\right) \cap f\left(V_{a}\right) \rightarrow g\left(f\left(U_{a}\right) \cap f\left(V_{a}\right)\right)
$$

is a restriction of $g$ and it is a homeomorphism because it is a composition of homeomorphisms. Therefore, $g: \mathrm{B} \rightarrow \mathrm{C}$ is étale. It clearly is a surjection. So Assumption 2.19 is satisfied.

Unless A is discrete, the constant map from A to a point is not étale, so Assumption 2.20 fails even if we exclude the empty space.

Let $\mathrm{G}$ be a basic groupoid and let $\left(\mathrm{X} ; \mathrm{m}_{\mathbf{X}} ; \mathrm{s}_{\mathbf{X}}\right)$ be a $\mathrm{G}$-action. We know from the proof of Lemma 8.5 that the following map

$$
\left(\mathrm{pr}_{1} ; \mathrm{m}_{\mathrm{X}}\right): \mathrm{X} \times_{\mathrm{sX}_{\mathrm{X}}, \mathrm{G}_{0}, \mathrm{r}} \mathrm{G}^{1} \stackrel{\sim}{\rightarrow} \mathrm{X} \times_{\mathrm{q}, \mathrm{X} / \mathrm{G}, \mathrm{q}} \mathrm{X}, \quad(x ; g) \mapsto(x ; x \cdot g),
$$

is a homeomorphism. Since the range and source maps are étale, the set of units $\mathrm{u}\left(\mathrm{G}^{0}\right)$ is open in $\mathrm{G}^{1}$. Therefore, the subset $\left(\mathrm{pr}_{1} ; \mathrm{m}_{\mathrm{X}}\right)\left(\mathrm{X} \times_{\mathrm{sX}_{\mathrm{X}}, \mathrm{G}_{0}, \mathrm{r}} \mathrm{u}\left(\mathrm{G}^{0}\right)\right)$ is open in $\mathrm{X} \times \times_{\mathrm{q}, \mathrm{X} / \mathrm{G}, \mathrm{q}} \mathrm{X}$. This subset is the diagonal $\left\{\left(x, x_{1}\right) \in \mathbf{X} \times \mathbf{X} \mid x=x_{1}\right\}$. Therefore, every element $(x ; x)$ of the diagonal has a neighbourhood $\left(V ; V^{\prime}\right)$ such that $\left(V ; V^{\prime}\right) \subset$ $\left\{\left(x, x_{1}\right) \in \mathrm{X} \times \mathbf{X} \mid x=x_{1}\right\}$. So any element $x \in \mathrm{X}$ has a neighbourhood $U=V \cap V^{\prime}$ such that $(U \times U) \cap\left(\mathbf{X} \times{ }_{\mathbf{q}, \mathbf{X} / \mathrm{G}, \mathrm{q}} \mathbf{X}\right)$ is the diagonal in $U$. This means that for $x, x_{1} \in U$, $\mathrm{q}(x)=\mathrm{q}\left(x_{1}\right)$ only if $x=x_{1}$. Thus $\mathrm{q}$ is injective on the open subset $U \subseteq \mathrm{X}$. Since $\mathrm{G}$ is an étale groupoid, its range and source maps are open. Hence $\mathrm{q}$ is open by Proposition 8.22 Its restriction to $U$ is injective, open and continuous, hence a homeomorphism onto an open subset of $X / G$. Therefore, it is étale. So Assumptions 5.63 and 5.66 are satisfied by Lemma 8.5

8.3. Categories of manifolds. The examples of a stronger pretopology considered in this subsection are defined in the categories of finite-dimensional manifolds $\left(\mathrm{Mfd}_{\text {fin }}\right)$; Hilbert manifolds $\left(\mathrm{Mfd}_{\mathrm{Hil}}\right)$; Banach manifolds $\left(\mathrm{Mfd}_{\mathrm{Ban}}\right)$; Fréchet manifolds $\left(\mathrm{Mfd}_{\text {Fré}}\right)$ and locally convex manifolds $\left(\mathrm{Mfd}_{\mathrm{lcs}}\right)$. Such manifolds are Hausdorff topological spaces that are locally homeomorphic to finite-dimensional vector spaces, Hilbert spaces, Banach spaces, Fréchet spaces, or locally convex topological vector spaces, respectively. The morphisms between all these types of manifolds are smooth maps. In each case, a stronger pretopology is defined by submersions.

Definition 8.25 ([23, Definition 4.4.8], [40, Appendix A]). Let $X$ and $Y$ be locally convex manifolds. A smooth map is a submersion if for each $x \in \mathrm{X}$, there is an open neighbourhood $V$ of $x$ in $\mathrm{X}$ such that $U=f(V)$ is open in $\mathrm{Y}$, and there are a smooth manifold $W$ and a diffeomorphism $V \cong U \times W$ that intertwines $f$ and the coordinate projection $\mathrm{pr}_{1}: U \times W \rightarrow U$.

Example 8.26. Let $\mathcal{C}$ be one of the categories $\mathrm{Mfd}_{\mathrm{fin}}, \mathrm{Mfd}_{\mathrm{Hil}}, \mathrm{Mfd}_{\mathrm{Ban}}, \mathrm{Mfd}_{\mathrm{Fre}}, \mathrm{Mfd}_{\mathrm{lcs}}$ considered above. Let $\mathcal{F}_{p}$ be the collection of all submersions in $\mathcal{C}$. Then $\left(\mathcal{C}, \mathcal{F}_{p}\right)$ is a category with partial covers. Isomorphisms are submersions, hence (1) holds. A composition of submersions is a submersion, thus (2) is satisfied. Let $\mathrm{pr}_{2}: \mathrm{X} \times_{f, \mathrm{Y}, g}$ $Z \rightarrow Z$ be a pull-back of a submersion $f: X \rightarrow Y$ along any smooth map $g: Z \rightarrow Y$. A submersion $f: \mathbf{X} \rightarrow \mathrm{Y}$ is open because it is locally open. Thus $\mathrm{pr}_{2}: \mathbf{X} \times_{f, \mathrm{Y}, g} \mathbf{Z} \rightarrow \mathbf{Z}$ is open because open maps form a stronger pretopology by Example 8.21 So $\operatorname{Im}(f)$ and $\operatorname{Im}\left(\mathrm{pr}_{2}\right)$ are open subspaces. It is clear that $\mathrm{X} \times_{f, \mathrm{Y}, g} \mathbf{Z} \cong \mathbf{X} \times_{f, \operatorname{Im}(f),\left.g\right|_{\operatorname{Im}\left(\mathrm{pr}_{2}\right)}}$ $\operatorname{Im}\left(\mathrm{pr}_{2}\right)$ and therefore, $\mathrm{pr}_{2}: X \times_{f, Y, g} Z \rightarrow \operatorname{Im}\left(\mathrm{pr}_{2}\right)$ is a pull-back of the surjective submersion $f: \mathbf{X} \rightarrow \operatorname{Im}(f)$ along $g: \mathbf{Z} \rightarrow \operatorname{Im}(f)$. Therefore, $\operatorname{pr}_{2}: \mathbf{X} \times_{f, Y, g} \mathbf{Z} \rightarrow$ 
$\operatorname{Im}\left(\mathrm{pr}_{2}\right)$ is a surjective submersion because surjective submersions form a pretopology ([47, Proposition 9.40]). So $\mathrm{pr}_{2}: \mathrm{X} \times_{f, \mathrm{Y}, g} \mathrm{Z} \rightarrow \mathrm{Z}$ is a submersion. Hence the condition (3) holds. In the proof of Proposition $9.40([4])$ it is shown that the pretopology defined by surjective submersions is subcanonical. That is, all surjective submersions are coequalisers. It is clear that a coequaliser is surjective. Therefore, a submersion is surjective if and only if it is a coequaliser. So covers are surjective submersions.

In all categories described in Example 8.26. Assumptions 2.14 and 2.15 hold by Proposition $9.40([4])$. In the categories $\mathrm{Mfd}_{\mathrm{fin}}, \mathrm{Mfd}_{\mathrm{Hil}}$ and $\mathrm{Mfd}_{\mathrm{Ban}}$ with such stronger pretopology, Assumptions 2.18 and 5.66 hold by Proposition 9.42 ([47]). In the category of Banach manifolds with the same stronger pretopology Assumption 5.63 is satisfied by Proposition $9.44([4])$. We have no information about these assumptions in other categories.

\section{REFERENCES}

[1] Michael Artin, Alexandre Grothendieck, and Jean-Louis Verdier, Théorie des topos et cohomologie étale des schémas. Tome 1: Théorie des topos, Lecture Notes in Mathematics, vol. 269, Springer, Berlin, 1972. Séminaire de Géométrie Algébrique du Bois-Marie 1963-1964 (SGA 4). MR 0354652

[2] John C. Baez, An introduction to $n$-categories, Category theory and computer science (Santa Margherita Ligure, 1997), Lecture Notes in Comput. Sci., vol. 1290, Springer, Berlin, 1997, pp. 1-33, doi: 10.1007/BFb0026978. MR 1640335

[3] J. Michael Boardman and Rainer M. Vogt, Homotopy invariant algebraic structures on topological spaces, Lecture Notes in Mathematics, vol. 347, Springer, Berlin, 197:MR 0420609

[4] Nicolas Bourbaki, Topologie générale. Chapitres 1 à 4, Éléments de mathématique, Hermann, Paris, 1971. MR 0358652

[5] Éléments de mathématique. Fasc. XXXVII. Groupes et algèbres de Lie. Chapitre II: Algèbres de Lie libres. Chapitre III: Groupes de Lie, Hermann, Paris, 1972. MR 0573068

[6] Michel Hilsum and Georges Skandalis, Morphismes K-orientés d'espaces de feuilles et fonctorialité en théorie de Kasparov (d'après une conjecture d'A. Connes), Ann. Sci. École Norm. Sup. (4) 20 (1987), no. 3, 325-390, available at http://www.numdam.org/item?id=ASENS_ 1987_4_20_3_325_0 MR925720

[7] Javier Bracho, Haefliger structures and linear homotopy, Trans. Amer. Math. Soc. 282 (1984), no. 2, 529-538, doi: 10.2307/1999250 MR 732104

[8] Mădălina Roxana Buneci, Morphisms of discrete dynamical systems, Discrete Contin. Dyn. Syst. 29 (2011), no. 1, 91-107, doi: 10.3934/dcds.2011.29.91. MR 2725283

[9] Mădălina Roxana Buneci and Piotr Stachura, Morphisms of locally compact groupoids endowed with Haar systems (2005), eprint. arXiv: 0511613.

[10] Alcides Buss and Ralf Meyer, Iterated crossed products for groupoid fibrations (2016), eprint. arXiv: 1604.02015

[11] Alcides Buss, Ruy Exel, and Ralf Meyer, Inverse semigroup actions as groupoid actions, Semigroup Forum 85 (2012), no. 2, 227-243, doi: 10.1007/s00233-012-9418-y. MR 2969047

[12] David Carchedi, Categorical properties of topological and diffentiable stacks, Ph.D. Thesis, Universiteit Utrecht, 2011, http://people.mpim-bonn.mpg.de/carchedi/Thesis_David_ Carchedi.pdf

[13] Henri Cartan (ed.), Ecole Normale Supérieure, Séminaire Henri Cartan, 2e année 1949/1950, Espaces fibrés et homotopie, 2nd ed., Secrétariat mathématique, Paris, 1956.

[14] Zhuo Chen and Zhang-Ju Liu, On (co-)morphisms of Lie pseudoalgebras and groupoids, J. Algebra 316 (2007), no. 1, 1-31, doi: 10.1016/j.jalgebra.2007.07.001 MR 2354851

[15] Lisa Orloff Clark, Classifying the types of principal groupoid $C^{*}$-algebras, J. Operator Theory 57 (2007), no. 2, 251-266, available at http://www.theta.ro/jot/archive/2007-057-002/ 2007-057-002-003.html. MR 2328998

[16] Maria Manuel Clementino and Dirk Hofmann, On limit stability of special classes of continuous maps, Topology Appl. 125 (2002), no. 3, 471-488, doi: 10.1016/S0166-8641(01)00293-0 MR 1935164

[17] Albrecht Dold, Partitions of unity in the theory of fibrations, Ann. of Math. (2) 78 (1963), 223-255, doi: 10.2307/1970341 MR 0155330

[18] Ieke Moerdijk and Janez Mrčun, Introduction to foliations and Lie groupoids, Cambridge Studies in Advanced Mathematics, vol. 91, Cambridge University Press, Cambridge, 2003. MR2012261 
[19] Geoff Goehle, Groupoid crossed products, Ph.D. Thesis, Dartmouth College, 2009, http: //search.proquest.com/docview/230889835. MR 2941279

[20] André Haefliger, Feuilletages sur les variétés ouvertes, Topology 9 (1970), 183-194, doi: 10.1016/0040-9383(70)90040-6 MR 0263104

[21] Otomar Hájek, Notes on quotient maps, Comment. Math. Univ. Carolinae 7 (1966), 319-323, available at http://dml.cz/handle/10338.dmlcz/105065 MR 0202118

[22] _ Correction to: "Notes on quotient maps", Comment. Math. Univ. Carolinae 8 (1967), 171, available at http://dml.cz/handle/10338.dmlcz/105100 MR 0210089

[23] Richard S. Hamilton, The inverse function theorem of Nash and Moser, Bull. Amer. Math. Soc. (N.S.) 7 (1982), no. 1, 65-222, doi: 10.1090/S0273-0979-1982-15004-2 MR 656198

[24] André Henriques, Integrating $L_{\infty}$-algebras, Compos. Math. 144 (2008), no. 4, 1017-1045, doi: 10.1112/S0010437X07003405 MR 2441255

[25] Astrid an Huef, The transformation groups whose $C^{*}$-algebras are Fell algebras, Bull. London Math. Soc. 33 (2001), no. 1, 73-76, doi: 10.1112/blms/33.1.73 MR 1798578

[26] Madeleine Jotz and Karl-Hermann Neeb, Closedness of the tangent spaces to the orbits of proper actions, J. Lie Theory 18 (2008), no. 3, 517-521, available at http://www.heldermann. $\mathrm{de} / \mathrm{JLT} / \mathrm{JLT} 18 / \mathrm{JLT} 183 / \mathrm{jlt} 18031 . \mathrm{htm}$ MR 2493051

[27] André Joyal, Quasi-categories and Kan complexes, J. Pure Appl. Algebra 175 (2002), no. 1-3, 207-222, doi: 10.1016/S0022-4049(02)00135-4 MR 1935979

[28] Anders Kock, Synthetic differential geometry, 2nd ed., London Mathematical Society Lecture Note Series, vol. 333, Cambridge University Press, Cambridge, 2006. MR 2244115

[29] Serge Lang, Fundamentals of differential geometry, Graduate Texts in Mathematics, vol. 191, Springer, New York, 1999. MR 1666820

[30] Tom Leinster, Basic Bicategories (1998), eprint. arXiv: math/9810017

[31] Kirill C. H. Mackenzie, General theory of Lie groupoids and Lie algebroids, London Mathematical Society Lecture Note Series, vol. 213, Cambridge University Press, Cambridge, 2005. MR 2157566

[32] Ernest A. Michael, Bi-quotient maps and Cartesian products of quotient maps, Ann. Inst. Fourier (Grenoble) 18 (1968), no. fasc. 2, 287-302 vii (1969), doi: 10.5802/aif.30 MMR 0244964

[33] _ A quintuple quotient quest, General Topology and Appl. 2 (1972), 91-138, doi: 10.1016/0016-660X(72)90040-2 MR 0309045

[34] _ Complete spaces and tri-quotient maps, Illinois J. Math. 21 (1977), no. 3, 716-733, available at http://projecteuclid.org/euclid.ijm/1256049022 MR 0467688

[35] Suliman Albandik, A colimit construction for groupoids, Ph.D. Thesis, Georg-AugustUniversität Göttingen, 2015, http://hdl.handle.net/11858/00-1735-0000-0028-87E8-C

[36] Ieke Moerdijk and Janez Mrčun, Lie groupoids, sheaves and cohomology, Poisson geometry, deformation quantisation and group representations, London Math. Soc. Lecture Note Ser., vol. 323, Cambridge Univ. Press, Cambridge, 2005, pp. 145-272, doi: 10.1017/CBO9780511734878 MR 2166453

[37] Ieke Moerdijk and Gonzalo E. Reyes, Models for smooth infinitesimal analysis, Springer, New York, 1991. MR 1083355

[38] Janez Mrcun, Stability and invariants of Hilsum-Skandalis maps, Ph.D. Thesis, Utrecht University, 1996, http://arxiv.org/abs/math/0506484

[39] Paul S. Muhly, Jean N. Renault, and Dana P. Williams, Equivalence and isomorphism for groupoid $C^{*}$-algebras, J. Operator Theory 17 (1987), no. 1, 3-22, available at http: //www.theta.ro/jot/archive/1987-017-001/1987-017-001-001.html. MR 873460

[40] Thomas Nikolaus, Christoph Sachse, and Christoph Wockel, A smooth model for the string group, Int. Math. Res. Not. IMRN 16 (2013), 3678-3721, doi: 10.1093/imrn/rns154 MR 3090706

[41] Behrang Noohi, Foundations of Topological Stacks I (2005), eprint. arXiv: math.AG/0503247

[42] _ Notes on 2-groupoids, 2-groups and crossed modules, Homology, Homotopy Appl. 9 (2007), no. 1, 75-106, available at http://projecteuclid.org/euclid.hha/1175791088 MR 2280287

[43] Richard S. Palais, On the existence of slices for actions of non-compact Lie groups, Ann. of Math. (2) 73 (1961), 295-323, doi: 10.2307/1970335 MR 0126506

[44] Jean Pradines, Morphisms between spaces of leaves viewed as fractions, Cahiers Topologie Géom. Différentielle Catég. 30 (1989), no. 3, 229-246 (English, with French summary). MR 1029626

[45] Dorette A. Pronk, Etendues and stacks as bicategories of fractions, Compositio Math. 102 (1996), no. 3, 243-303, available at http://www.numdam.org/item?id=CM_1996_-102_3_243_0 MR 1401424

[46] Marc A. Rieffel, Applications of strong Morita equivalence to transformation group $C^{*}$-algebras, Operator algebras and applications, Part I (Kingston, Ont., 1980), Proc. 
Sympos. Pure Math., vol. 38, Amer. Math. Soc., Providence, RI, 1982, pp. 299-310, doi: 10.1090/pspum/038.1/679709 MR 679709

[47] Ralf Meyer and Chenchang Zhu, Groupoids in categories with pretopology, Theory Appl. Categ. 30 (2015), 1906-1998, available at http://www.tac.mta.ca/tac/volumes/30/55/30-55abs. html.

[48] David Michael Roberts, Internal categories, anafunctors and localisations, Theory Appl. Categ. 26 (2012), 788-829, available at http://www.tac.mta.ca/tac/volumes/26/29/26-29abs.html MR 3065944

[49] Ruy Exel, Partial dynamical systems, Fell bundles and applications, Mathematical Surveys and Monographs, vol. 224, Amer. Math. Soc., Providence, RI, 2017. MR3699795

[50] Graeme Segal, Classifying spaces and spectral sequences, Inst. Hautes Études Sci. Publ. Math. 34 (1968), 105-112, available at http://www.numdam.org/item?id=PMIHES_1968_34_105_0 MR 0232393

[51] Dana P. Williams, Crossed products of $C^{*}$-algebras, Mathematical Surveys and Monographs, vol. 134, Amer. Math. Soc., Providence, RI, 2007. MR 2288954

[52] Christoph Wockel and Chenchang Zhu, Integrating central extensions of Lie algebras via Lie 2-groups (2012), eprint. arXiv: 1204.5583 .

[53] Jesse Wolfson, Descent for n-bundles (2013), eprint. arXiv: 1308.1113

[54] $\mathrm{Du} \mathrm{Li}$, Higher groupoid actions, bibundles, and differentiation, Ph.D. Thesis, Georg-AugustUniversität Göttingen, 2014, http://hdl.handle.net/11858/00-1735-0000-0022-5F4F-A

[55] Marco Zambon and Chenchang Zhu, Contact reduction and groupoid actions, Trans. Amer. Math. Soc. 358 (2006), no. 3, 1365-1401, doi: 10.1090/S0002-9947-05-03832-8. MR 2187657 